\title{
Thèse
}

Static and Dynamic Stiffness Analysis of Cable-Driven Parallel Robots

Thèse soutenue le 11.03.2015

devant le jury composé de :

Serge Samper

Professeur, Institut de Physique de Rennes / président Jacques Gangloff

Professeur, Telecom Physique Strasbourg / rapporteur

Stéphane Caro

Chargé de Recherche CNRS HDR, IRCCYN, Ecole Centrale de Nantes / rapporteur

Cédric Baradat

Ingénieur, Directeur Technique Tecnalia / examinateur

\section{Georges Dumont}

Professeur ENS Rennes, IRISA / examinateur

Marc Gouttefarde

Chargé de Recherche CNRS, LIRMM, Montpellier / examinateur

Eric Courteille

Maître de Conférences INSA-Rennes / Co-encadrant de thèse

\section{Eric Ragneau}

Professeur INSA-Rennes / Directeur de thèse 



\section{Static and Dynamic Stiffness Analysis of Cable-Driven Parallel Robots}

Han Yuan
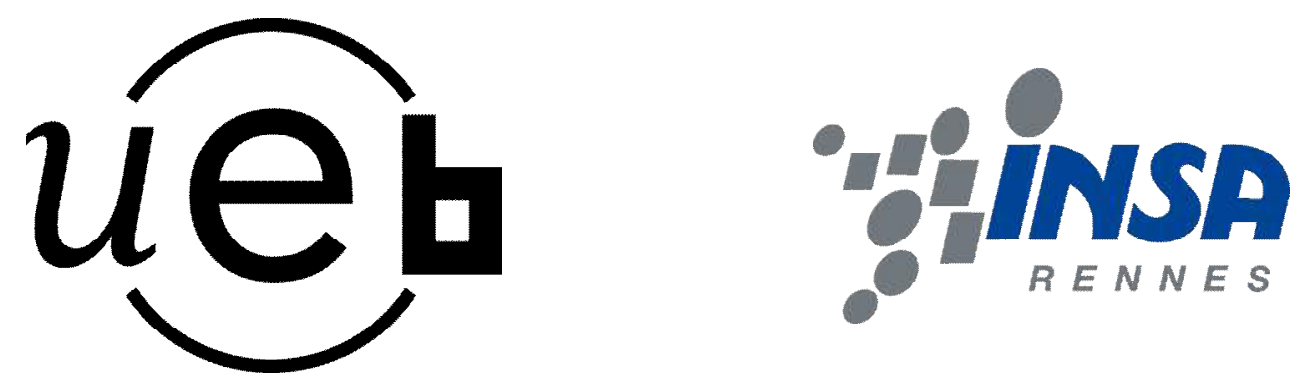

To Lily,

To my parents,

To all my Friends. 



\section{Acknowledgements}

I would like to express my special appreciation and thanks to my advisors Mr. Eric RAGNEAU, Mr. Eric COURTEILLE and Mr. Dominique DEBLAISE for giving me the opportunity to work in a talented research team on such an interesting and rewarding subject. I would like to thank you for your patience, continuous encouragement, invaluable insights and excellent instructions. Your advice on both research as well as on my career have been priceless. I would also like to thank my committee members, Mr.Serge Samper, Mr. Jacques Gangloff, Mr. Stéphane Caro, Mr. Cédric Baradat, Mr. Georges Dumont, and Mr. Marc Gouttefarde. Thanks for your inspiring suggestions and encouraging feedbacks.

I would especially like to thank my colleagues in PSM team. Thank you very much for all the great conversations and good time we have had. Special thanks to Mr. Marc Gouttefarde, Mr. Cédric BARADAT and the CoGiRo team of LIRMM for giving us supports in the experiments on the CoGiRo.

I would like to thank my family. Words can not express how grateful I am to my loving parents. A great thanks to my beloved Lily. Thank you for your eneless supporting and understanding during my doctoral research. I would also like to thank all of my friends who supported me and incented me to strive towards my goal.

Finally I greatly acknowledge the financial support of this work provided by China Scholarship Council (CSC). 



\section{General Introduction}

Cable-Driven Parallel Robots (CDPRs) are a special variant of traditional rigid-link parallel robots. They use flexible cables, instead of rigid links, to connect the movable end-effector and the fixed base. The end-effector is manipulated by changing the lengths of the cables that are actuated by the fixed motors and winches.

As a kind of parallel robots, CDPRs have advantages in load capacity, stiffness, efficiency and so on. Furthermore, CDPRs overcome the major weakness of rigid-link parallel robots: workspace. Unlike rigid links, cable lengths can vary in a wide range, which enlarges the workspace of CDPRs. These characteristics have attracted a lot of interest of researchers in the past few decades [Merlet 2006; Gouttefarde+ 2006; Gosselin+ 2011; Gouttefarde+ 2012; Weber+ 2014; Pott+ 2013; Bruckmann+ 2006; Arsenault 2013].

However, due to the compliance of cables, the stiffness analysis of CDPRs becomes a vital concern [Gouttefarde+ 2012; Riehl+ 2009], especially for suspended configuration. Stiffness has a significant effect on the static and dynamic behaviors of CDPRs, such as kinematics, positioning accuracy, force distribution, vibration and control [Gosselin 1990; Merlet 2006]. Deficient static stiffness can decrease the positioning accuracy of CDPRs, and bad dynamic stiffness characteristics can lead to vibration and long settling time. This thesis will focus on the static and dynamic stiffness analyses of CDPRs aiming to improve the static positioning and trajectory tracking accuracies of CDPRs.

For the static stiffness analysis of CDPRs, the compliance of the driving cables is the major factor that affects the positioning accuracy of CDPRs. Considering the physical cable characteristics, the compliance of cables mainly has two sources. One is the axial stiffness of the cables, which is associated with the elastic material modulus and the cable structure. The other is the sag-introduced flexibility, which comes from the effect of cable weight on the static cable profile. The sag-introduced flexibility corresponds to the gravitational potential energy stored in the cable.

To study the CDPRs stiffness behavior, many previous studies only consider the axial stiffness of the cables [Verhoeven+ 1998; Dagalakis+ 1989; Kawamura+ 1995; Kawamura+ 2000; Behzadipour+ 2006; Korayem+ 2007; Bedoustani+ 2008; Vafaei+ 2011; Khosravi+ 
2013]. In these researches, sag-introduced flexibility is neglected, and massless spring is used as the cable model. The spring cable model is simple and suitable for real-time applications. Another well known cable model is the static sagging cable model deriving from civil engineering [Irvine 1992]. It is used in several previous researches [Kozak+ 2006; Riehl+ 2009; Gouttefarde+ 2012; Sandretto+ 2013b; Arsenault 2013]. The sagging cable model considers the axial stiffness of the cables and the sag-introduced flexibility. It is more accurate than the spring cable model in the static analysis of CDPRs. In previous researches [Kozak+ 2006; Riehl+ 2009; Arsenault 2013], the effect of cable sag on the static stiffness of CDPRs is only verified by numerical simulations. Experimental verification of the static stiffness is performed only on single cables in [Kozak+ 2006; Irvine 1992]. To our best knowledge, the only experimental validation of the sagging cable model on a complete CDPR is presented in [Nguyen+ 2013].

The static sagging cable model is firstly introduced in this thesis. Based on the sagging cable model, the static pose error of the end-effector is defined and the variation of the end-effector pose error with the external load is used to evaluate the static stiffness of CDPRs. The sagging cable model and the effect of cable sag on the static positioning accuracy of CDPRs are verified through the experiments on a 6-DOF CDPR prototype.

The vibration of CDPRs can be affected by the compliance of the driving cables, the actuators and the end-effector. Compared with cables, the compliance of the actuators and the end-effector is much lower and therefore can be neglected. Thus the compliance of cables is the primary reason for the vibration of CDPRs. The rigid-body modes of the end-effector suspended on the stiffness of the cables and the coupling with the cable vibration should be considered in the dynamic analysis of CDPRs. Most of the previous researches [Diao+ 2009; Ma+ 2005; Tang+ 2013; Weber+ 2014] use linear massless axial springs as the dynamic cable model, which only considers cable elasticity, while neglecting the effect of cable dynamics on the system vibration. Some other researches [Du+ 2012; Du+ 2013] consider the effect of cable dynamics through the finite element cable model, which uses distributed mass points and ideal lines between them to simulate continuous cables. Thus an important issue of the dynamic analysis of CDPRs is to find out whether the cable resonances and vibration affect the dynamics of CDPRs. Moreover, how to set up a complete dynamic cable model that considers the cable dynamics, the end-effector vibration and their coupling is still a challenge.

The Dynamic Stiffness Matrix (DSM) method is used to formulate the dynamic stiffness matrix of an inclined sagging cable in this thesis. The DSM method is used to solve the vibration problems of structures. It is often regarded as an exact method, because the 
DSM is based on the exact shape functions obtained from the exact solution of the element differential equations [Ansell 2005]. Based on the DSM method, a new dynamic model of CDPRs is proposed with considering the coupling of cable dynamics and end-effector vibrations. The dynamic stiffness matrix of CDPRs is computed according to the dynamic cable model and geometric relationship. This dynamic matrix is an assemblage of the dynamic stiffness matrix of all the driving cables. It considers the cable elasticity and the effect of cable dynamics on the system dynamics. With this dynamic stiffness matrix, the oscillating equations of the end-effector around a static equilibrium are formulated through the Lagrange's equations. Dynamic response functions of the end-effector under a harmonic excitation are used to identify the natural frequencies of CDPRs, and to study the coupling of the cable dynamics and the end-effector vibrations. A CDPR prototype with 6-DOF driven by 8 cables, the CoGiRo [Lamaury 2013], is used in the dynamic experimental validation. Modal experiments, free vibration experiments and trajectory experiments are carried out to validate the introduced dynamic cable model and the proposed dynamic stiffness model of CDPRs, also analyze the coupling between the cable dynamics and the end-effector vibration.

Besides static and dynamic stiffness analysis, the proposed models are applied on the force distribution of redundant actuated CDPRs. Due to the actuation redundancy, there exists infinite solutions of cable forces to balance a given wrench applied on the endeffector. As a consequence, one important design issue for redundant actuated CDPRs is the identification and the calculation of feasible cable force distribution. Previous studies on this issue [Gosselin+ 2011; Mikelsons+ 2008; Pott+ 2009; Khosravi+ 2013; Oh+ 2005; Bruckmann+ 2006; Kawamura+ 1995; Fang+ 2004; Hassan+ 2008; Lim+ 2011] usually neglect the effect of cable weight on the cable profile and/or the cable elasticity, where cables are assumed as massless straight lines. This assumption is not accurate, especially for CDPRs with heavy and/or long cables. Inaccurate cable forces computation can affect the performances of CDPRs such as the positioning accuracy and the trajectory tracking due to vibration [Yuan+ 2015].

Another important issue is the determination of the lower-boundary of cable forces. In fact, in order to keep all the cables in tension, a positive lower-boundary of cable forces is used as a constraint in the identification problem of force distribution for redundant CDPRs. Small cable forces tend to cause cable sag and decrease cable stiffness [Yuan+ 2015; Arsenault 2013]. In some case, an important cable sag can even cause the end-effector to become under-constrained, and make the robot out of control [Gosselin+ 2011]. On another hand, the internal forces of all the driving cables can be increased by raising the lower-boundary used in the force distribution computation. The cable sag is decreased and 
cable vibration is reduced. Thus the performances of CDPRs are improved. But this can directly lead to a significant growth in motor torque and energy consumption, which enlarges both the manufacturing and the operating cost of CDPRs. In previous researches, the value for the lower-boundary of cable forces is usually chosen arbitrarily. As far as we know, there is no literature on the determination of the lower-boundary of cable forces.

The force distribution method considering the effect of cable weight on the static cable profile is presented in this thesis. With cable sag, the kinematics and force distribution of CDPRs are coupled. The proposed method solves the coupling problem by using optimization algorithms. Methods on the determination of the lower-boundary of cable forces are presented, and a new pose-dependent force boundary method is proposed based on the dynamic cable model presented in this thesis. The lower-boundary of each driving cable is calculated for every pose of the end-effector along a trajectory. Compared with the traditional fixed lower-boundary method, the proposed pose-dependent lower-boundary method can give out much more suitable force boundaries for every cable. Thus it can guarantee the cable performance according to the design requirement while not stretch the cable too much.

This thesis is organized in 6 chapters.

In Chapter 1, literature review is made. The advantages of CDPRs are presented and the classifications of CDPRs are made. We discuss the problematic and current researches on the static and dynamic stiffness analysis of CDPRs.

In Chapter 2, cable modeling is introduced. The static cable model is studied in section 2.1. The profile of a static sagging cable is described through a set of non-linear equations. Then some parameters associated with the static characteristics are computed, such as the coordinates of the end point, the chord length, the inclined angle, etc. These parameters are useful for the static analysis of sagging cable, and they are also important in the analysis of cable dynamics. And then, based on the static cable model, the static stiffness matrix of a sagging cable is formulated, and the sag-introduced flexibility is introduced. Besides the static cable model, the dynamic cable model is given in section 2.2. The dynamic stiffness matrix of a horizontal sagging cable is introduced, then it is expended to an inclined sagging cable. After cable modeling, an example is given in section 2.3 in order to illustrate how to analyze the static and dynamic characteristics of an inclined sagging cable by using the introduced cable models. Static cable characteristics are presented including the plots of the static cable profiles, the static compliance and stiffness matrix. Then cable dynamics is analysis through the amplitude variation of the trace of the dynamic stiffness matrix.

In Chapter 3, stiffness modeling of CDPRs is presented. The static stiffness model of CDPRs is developed in section 3.2. The inverse and direct kinematics of CDPRs are firstly 
presented with considering both the cable elasticity and the effect of cable weight on the cable profile. Then the static pose error of the end-effector is defined based on the direct kinematic model of CDPRs and the variation of the pose error of the end-effector with the external load is used to evaluated the static stiffness of CDPRs. The dynamic stiffness model of CDPRs is developed in section 3.3. The dynamic stiffness matrix of CDPRs is formulated, which contains all the dynamic characteristics of the driving cables. Then the dynamic stiffness matrix is used to develop the oscillating model of the end-effector around a static equilibrium. Based on the oscillating model, the dynamic response functions of the end-effector under a harmonic excitation are calculated, which enables to identify the natural frequencies of CDPRs and study the effect of cable dynamics on the system vibrations. In section 3.4, a simulation of a 6-DOF suspended CDPR driven by 8 cables used for the pick-and-place application is chosen as an example in order to illustrate the static and dynamic stiffness modeling of CDPRs through the proposed methods.

In Chapter 4, experimental validation is carried out to show the relevance of the proposed models on improving the performances of CDPRs in terms of design and control. Two CDPR prototypes are firstly described in section 4.1. Static experiments are made and the static stiffness of the 6-cable CDPR prototype is studied in section 4.2. The static sagging cable is validated and the effect of external load on the static stiffness of CDPR is analyzed. Dynamic experiments are made on the 8-cable prototype CoGiRo to validate the dynamic stiffness modeling and analyze the coupling between cable dynamics and end-effector vibration: including the dynamic modal experiments (section 4.3), the free vibration analysis at an emergency stop during a trajectory (section 4.4.1) and the dynamic experiment along a whole trajectory (section 4.4.2).

In Chapter 5, the proposed methods are applied to the force distribution of redundant actuated CDPRs. Kinematics modeling and force distribution of CDPRs with considering the effect of cable sag are presented in section 5.1. Then methods on the determination of the lower-boundary of cable forces are proposed in section 5.2, including the calculation of the fixed boundary and the pose-dependent boundary. Simulations on a 6-DOF CDPR driven by 8 cables are presented as an example in section 5.3 to illustrate how to determinate the lower-boundary of cable forces and calculate the force distribution with the proposed methods.

Chapter 6 summarizes the results and makes perspectives on the future work. 



\section{Introduction Générale}

Les robots parallèles à câbles utilisent des câbles flexibles à la place des chaines cinématiques rigides que l'on trouve sur les robots parallèles traditionnels. La pose (position et orientation) de la nacelle dépend donc de la longueur des câbles qui peut être ajustée par des enrouleurs motorisés fixés sur la base du robot.

En plus de posséder les avantages classiques des robots parallèles traditionnels, à savoir la rigidité, la précision et de fortes capacités de chargement, les robots parallèles à câbles possèdent aussi un large espace de travail. C'est pour toutes ces raisons que depuis la dernière décennie, les activités de recherches sur cette thématique sont nombreuses [Merlet 2006; Gouttefarde+ 2006; Gosselin+ 2011; Gouttefarde+ 2012; Weber+ 2014; Pott+ 2013; Bruckmann+ 2006; Arsenault 2013].

Toutefois, en raison de la flexibilité des câbles, la rigidité des robots parallèles à câbles devient une préoccupation importante [Gouttefarde+ 2012; Riehl+ 2009], en particulier pour les robots parallèles à câbles suspendus. Cette rigidité a aussi un impact sur les caractéristiques suivantes de ces structures : les comportements statique et dynamique, la précision de pose, les vibrations, le contrôle et la distribution des forces dans le cas des structures redondantes [Gosselin 1990; Merlet 2006].

Une rigidité statique insuffisante peut diminuer la précision de pose, et de mauvaises caractéristiques en termes de rigidité dynamique peuvent conduire à des vibrations et à une durée de stabilisation plus importante.

Cette thèse se concentrera sur l'étude des rigidités statique et dynamique des robots parallèles à câbles afin d'en améliorer la précision de pose ainsi que la précision dans le suivi de trajectoires dans le cas d'applications nécessitant de fortes dynamiques. 


\section{Modélisation et analyse de la rigidité statique des robots par- allèles à câbles}

La flexibilité des câbles est le facteur le plus influent dans l'analyse de la rigidité statique des robots parallèles à câbles et est à l'origine d'erreur de pose de la nacelle.

La flexibilité des câbles à deux origines :

- la rigidité axiale qui est directement liée au module d'élasticité du câble.

- la flexibilité introduite par le profil du câble qui, lorsqu'il est suspendu entre deux points prend la forme d'une chaînette élastique, dont le profil dépend du poids du câble et donc directement lié à l'énergie potentielle gravitationnelle stockée dans le câble.

Dans de nombreuses études, la flexibilité liée à la chaînette élastique est négligée et seule la rigidité axiale du câble est prise en compte [Verhoeven+ 1998; Dagalakis+ 1989; Kawamura+ 1995; Kawamura+ 2000; Behzadipour+ 2006; Korayem+ 2007; Bedoustani+ 2008; Vafaei+ 2011; Khosravi+ 2013]. Le câble est alors modélisé comme un ressort sans masse. Ce modèle est simple et bien adapté pour les applications en temps réel.

Un autre modèle de câble bien connu est le modèle du câble pesant élastique utilisé dans le milieu du génie civil [Irvine 1992]. Ce modèle est repris et adapté aux robots parallèles à câbles [Kozak+ 2006; Riehl+ 2009; Gouttefarde+ 2012; Sandretto+ 2013b; Arsenault 2013]. Le modèle de câble pesant élastique prend en compte la rigidité axiale et la flexibilité liée à la chaînette élastique. Pour une étude en statique d'un robot parallèle à câbles, le modèle de câble pesant est plus précis que le modèle de ressort sans masse. Dans les précédentes recherches [Kozak+ 2006; Riehl+ 2009; Arsenault 2013], l'effet de la chaînette élastique sur la rigidité statique d'un robot parallèle à câbles n'est vérifié que par des simulations numériques. Des validations expérimentales du modèle de câble pesant sont proposées sur un unique câble dans [Kozak+ 2006; Irvine 1992] et à notre connaissance, une seule validation expérimentale est proposée sur un robot parallèle à câbles dans [Nguyen+ 2013].

Dans cette thèse, le modèle de câble pesant est rappelé puis, sur la base de ce modèle, l'erreur de pose de la nacelle est définie comme un nouvel indice de performance de la raideur statique d'un robot parallèle à câbles. Le modèle de câble pesant est validé expérimentalement sur un prototype de robot parallèle à câbles ayant 6 DOF. 


\section{Analyse et modélisation de la rigidité dynamique des robots parallèles à câbles}

Une question importante dans l'analyse des robots parallèles à câbles est de savoir si les vibrations des câbles ainsi que leurs modes de résonance affectent la dynamique de la structure. A ce jour, un modèle dynamique complet qui intégrerait la dynamique des câbles, les vibrations de la nacelle et le couplage entre les câbles et la nacelle est toujours un défi.

Les robots parallèles à câbles peuvent être soumis à des vibrations pouvant affecter les câbles, les actionneurs et la nacelle. Par rapport aux câbles, les compliances des actionneurs et de la nacelle sont beaucoup plus faibles et peuvent donc être négligées. Ainsi, la compliance des câbles est la principale raison de la vibration des robots parallèles à câbles. L'analyse dynamique des robots parallèles à câbles doit prendre en compte les vibrations des câbles, les modes de corps rigide de la nacelle suspendue sur la raideur des câbles, et les liaisons entre les câbles et la nacelle. Dans la plupart des recherches antérieures [Diao+ 2009; Ma+ 2005; Tang+ 2013; Weber+ 2014], un modèle de câble sans masse est utilisé dans le modèle dynamique de la structure, ce qui ne permet pas de prendre en compte l'effet dynamique des câbles dans la vibration de la structure, c'est à dire l'impact des modes de résonances des câbles eux-mêmes. D'autres recherches [Du+ 2012; Du+ 2013] intègrent l'effet de la dynamique des câbles en considérant un modèle éléments finis qui utilise des points affectés d'une fraction de la masse du câble pour simuler le câble.

Dans cette thèse, la méthode dynamique matrice de rigidité (DSM for Dynamic Stiffness Matrix) est utilisée pour formuler la matrice de rigidité dynamique d'un câble pesant incliné. La méthode DSM est utilisée pour résoudre les problèmes vibratoires des structures. Cette méthode est considérée comme une méthode exacte, car basée sur les fonctions de forme obtenues à partir de la solution exacte des équations différentielles [Ansell 2005]. Un nouveau modèle dynamique de robot parallèle à câbles est alors proposé en prenant en compte le couplage de la dynamique des câbles et les vibrations de l'effecteur. La matrice de rigidité dynamique des robots parallèles à câbles est calculée selon le modèle dynamique de chaque câble et des relations géométriques. Cette matrice est un assemblage des matrices de rigidité dynamique de tous les câbles d'entraînement. Le modèle complet considère alors l'élasticité des câbles et l'effet de la dynamique des câbles sur la dynamique du système. Les équations d'équilibre vibratoire de l'organe terminal autour d'un équilibre statique sont formulées à partir du formalisme de Lagrange. Les Fonctions de Réponse en Fréquence (FRF) de la nacelle sous une excitation harmonique sont alors utilisées pour identifier les fréquences naturelles des robots parallèles à câbles, et ainsi étudier le couplage de la dynamique de 
câbles et les vibrations de la nacelle.

Le prototype COGIRO [Lamaury 2013] est utilisé pour les validations expérimentales en dynamique. Cette structure est actionnée par huit câbles et possède 6 degrés de liberté. Ces validations portent sur des essais d'analyse modale, des essais de vibrations en régime libre et en trajectoire et permettent de valider les modèles dynamiques des câbles et de la structure complète. Le couplage entre la dynamique des câbles et celle de la nacelle est aussi analysée.

\section{Application des méthodes proposées sur le calcul de la ré- partition des forces dans les câbles}

Outre l'analyse des rigidités statique et dynamique, les modèles proposés sont utilisés dans le calcul de la distribution des forces dans le cas des structures redondantes. Les précédentes études [Gosselin+ 2011; Mikelsons+ 2008; Pott+ 2009; Khosravi+ 2013; Oh+ 2005; Bruckmann+ 2006; Kawamura+ 1995; Fang+ 2004; Hassan+ 2008; Lim+ 2011] utilisent un modèle où les câbles sont supposés sans masse et de raideur infinie. Ces hypothèses présentent des limites dans le cas de câbles lourds et/ou longs. Une mauvaise distribution des forces peut occasionner des vibrations dans les câbles et ainsi affecter les performances de la structure en termes de précision de pose et de suivi de trajectoire [Yuan+ 2015].

Une autre question importante est la détermination de la limite inférieure des forces dans les câbles. Pour les structures redondantes, afin de garder tous les câbles en tension, une limite minimale positive est utilisée comme une contrainte pour l'identification de la distribution des forces. Une limite trop faible peut provoquer une déformation très importante du câble et ainsi agir sur sa rigidité [Yuan+ 2015; Arsenault 2013]. Dans des cas extrêmes la structure peut devenir sous-contrainte et ne plus être contrôlable [Gosselin+ 2011]. Afin de palier à cette problématique, la valeur de la limite minimale peut être augmentée. Dans ce cas la déformation du câble et les vibrations sont réduites, ce qui accroît les performances de la structure, mais cela peut conduire à d'importantes augmentations du couple moteur et donc de la consommation énergétique. Dans les travaux précédents, la valeur de cette limite inférieure est choisie arbitrairement, et à notre connaissance, il n'y a pas de littérature relative à la détermination de cette limite inférieure.

Dans cette thèse, la méthode de distribution des forces est basée sur l'utilisation d'un critère de flèche lié à la déformation du câble. La valeur de cette flèche dépend à la fois de la position de la nacelle et de la répartition des forces dans les câbles. Un algorithme 
d'optimisation est utilisé pour résoudre ce problème de couplage. Une nouvelle méthode de détermination de la limite inférieure de la force est présentée. Cette méthode est basée sur le modèle dynamique du câble pesant. Pour chacun des câbles de la structure, la valeur de la limite inférieure de la force est calculée et varie tout au long de la trajectoire. En comparaison avec la méthode traditionnelle de calcul de la distribution des forces, cette nouvelle approche permet de garantir la performance de chaque câble en fonction du critère de conception retenu sur tout l'espace de travail.

\section{Organisation de la thèse}

Cette thèse est organisée en six chapitres. Tout d'abord, une revue de la littérature est faite au chapitre 1. Les avantages et inconvénients des robots parallèles à câbles sont présentés et la classification de ces structures est faite. Par la suite, nous discutons des recherches actuelles sur les problématiques liées à l'analyse des rigidités statique et dynamique des robots parallèles à câbles.

Dans le chapitre 2, la modélisation du câble est introduite. Le modèle statique de câble pesant est étudié dans la section 2.1. Le profil de déformation statique d'un câble pesant est présenté à partir d'un ensemble d'équations non-linéaires. Ensuite, certains paramètres associés aux caractéristiques statiques sont calculés, telles que les coordonnées du point d'extrémité, la longueur de corde, l'angle d'inclinaison, etc... Ces paramètres sont utiles pour les analyses statique et dynamique du câble pesant. Puis, sur la base de ce modèle, la matrice de raideur statique d'un câble pesant est présentée. Le modèle dynamique de câble pesant est donné à la section 2.2. La matrice de raideur dynamique d'un câble pesant horizontal est d'abord introduite, puis elle est généralisée à un câble pesant incliné. Après la modélisation du câble, un exemple est donné dans la section 2.3 afin d'illustrer l'analyse des caractéristiques statiques et dynamiques d'un câble pesant incliné.

Dans le chapitre 3, la modélisation de la raideur des robots parallèles à câbles est présentée. Le modèle de raideur statique est développé dans la section 3.2. Les modèles cinématiques direct et inverse des robots parallèles à câbles sont présentés en prenant en compte du modèle de câble pesant. Ensuite, l'erreur de pose statique de la nacelle est définie sur la base du modèle cinématique direct et le déplacement de la nacelle dû à un chargement extérieur est utilisé pour évaluer la raideur statique des robots parallèles à câbles. Le modèle de raideur dynamique est développé dans la section 3.3. La matrice de raideur dynamique est présentée, elle intègre toutes les caractéristiques dynamiques des câbles pesants. Ensuite, la matrice de raideur dynamique est utilisée pour développer le modèle vibratoire de la 
nacelle autour d'un équilibre statique. Basé sur le modèle oscillant, les FRF de la nacelle sous une excitation harmonique sont calculées, ce qui permet d'identifier les fréquences naturelles des robots parallèles à câbles et d'étudier l'effet de la dynamique des câbles sur les vibrations du système. Dans la section 3.4, un robot parallèle à câbles suspendu à 6 degrés de liberté entraîné par 8 câbles utilisé pour des applications pick-and-place est choisi comme exemple pour illustrer la modélisation de rigidité statique et dynamique à travers les méthodes proposées.

Dans le chapitre 4, une validation expérimentale est effectuée pour démontrer la pertinence des modèles proposés sur l'amélioration des performances de robots parallèles à câbles en termes de conception et de contrôle. Deux prototypes sont d'abord décrits dans la section 4.1. La rigidité statique du prototype procédant 6 DOF est validé dans la section 4.2. Dans la section 4.3 des validations sur l'analyse de la rigidité dynamique et le couplage entre la dynamique des câbles et les vibrations de la nacelle sont faites. Dans la section 4.4 des analyses de vibrations libres suite à un arrêt d'urgence ainsi que des analyses vibratoires en suivi de trajectoire sont réalisées.

Dans le chapitre 5, les méthodes proposées sont appliquées au calcul de la distribution des forces dans le cas de robots parallèles à câbles redondants. Le modèle de calcul de la distribution des forces dans les câbles qui intègre l'effet de la chainette élastique est présenté dans la section 5.1. Le calcul de la limite inférieure des forces dans les câbles dépendant de la pose de la nacelle est présenté à la section 5.2. Des simulations réalisées à partir d'une structure possédant 8 câbles sont présentées dans la section 5.3.

Le chapitre 6 donne les conclusions et les perspectives des travaux de recherche présentées dans ce mémoire. 


\section{Contents}

Contents $\quad$ xxi

$\begin{array}{ll}\text { List of Figures } & \text { xxv }\end{array}$

\begin{tabular}{ll} 
List of Tables & xxix \\
\hline
\end{tabular}

1 Introduction 1

1.1 Presentation of CDPRs . . . . . . . . . . . . . . 1

1.1.1 Advantages and applications of CDPRs . . . . . . . . . 3

1.1.2 Classification of CDPRs . . . . . . . . . . . . 7

1.2 Problematic and current researches . . . . . . . . . . . . . . . 9

1.2.1 Cable force analysis . . . . . . . . . . . . . 9

1.2 .2 Stiffness analysis . . . . . . . . . . . . . . 10

1.3 Objectives of this thesis . . . . . . . . . . . . . . 13

2 Static and Dynamic Modeling of Cables 17

2.1 Static cable modeling . . . . . . . . . . . . . . . . . . . 19

2.1.1 Profile of a static sagging cable . . . . . . . . . . . . 19

2.1.2 Calculation of the static cable parameters . . . . . . . . . 21

2.1.3 Static stiffness matrix of a sagging cable . . . . . . . . . 23

2.2 Dynamic cable modeling . . . . . . . . . . . . . . . 25

2.2.1 Notifications and assumptions . . . . . . . . . . 25

2.2.2 Dynamic stiffness matrix of a horizontal sagging cable . . . . . . 25

2.2.3 Dynamic stiffness matrix of an inclined sagging cable . . . . . . 27

2.3 A numerical example . . . . . . . . . . . . . . . . . . 29

2.3.1 Static cable characteristics . . . . . . . . . . . . . . . 29

2.3.2 Dynamic cable characteristics . . . . . . . . . . . . . 32

2.4 Summary of the chapter . . . . . . . . . . . . . . . . 34 
3 Static and Dynamic Stiffness Modeling of CDPRs 35

3.1 Problem description . . . . . . . . . . . . . . . . 36

3.2 Static stiffness modeling of CDPRs . . . . . . . . . . . . . . . 37

3.2.1 Kinematic modeling . . . . . . . . . . . . . . . . 38

3.2.2 Static pose error definition . . . . . . . . . . . . . . 40

3.2.3 Static stiffness evaluation . . . . . . . . . . . . . . . . 42

3.3 Dynamic stiffness modeling of CDPRs . . . . . . . . . . . . . . . . 42

3.3.1 Computation of the dynamic stiffness matrix of CDPRs . . . . . . . 43

3.3.2 Oscillating model of the end-effector around a static equilibrium . . 44

3.3.3 Natural frequency identification . . . . . . . . . . . . . 46

3.4 A numerical Example . . . . . . . . . . . . . . . . . . . . . . . . . 48

3.4.1 Description of the studied CDPR . . . . . . . . . . . . . 48

3.4.2 Static stiffness modeling of the studied CDPR . . . . . . . . . . 49

3.4.3 Dynamic stiffness modeling of the studied CDPR . . . . . . . . . 52

3.5 Summary of the chapter . . . . . . . . . . . . . . . . . 56

4 Experimental Validation and Stiffness Analysis $\quad 57$

4.1 Description of the CDPR prototypes . . . . . . . . . . . . 58

4.1.1 The 6-cable CDPR prototype . . . . . . . . . . . . . . 58

4.1.2 The 8-cable CDPR prototype . . . . . . . . . . . . . . 60

4.2 Static experiments and static stiffness analysis . . . . . . . . . . 61

4.2.1 Static experimental setup . . . . . . . . . . . . . . . 61

4.2.2 Static experimental results and analysis . . . . . . . . . . . 62

4.3 Modal experiments and dynamic stiffness analysis . . . . . . . . . . . . . . 64

4.3.1 Verification of the static cable forces . . . . . . . . . . . . 65

4.3 .2 Experimental setup . . . . . . . . . . . . . . . . 67

4.3.3 Experimental results and discussions . . . . . . . . . . . . 68

4.4 Dynamic trajectory experiments and dynamic stiffness analysis . . . . . . . 75

4.4.1 Free vibration experiment at an emergency stop . . . . . . . . . 75

4.4 .2 Experiment along a trajectory . . . . . . . . . . . . 79

4.5 Summary of the chapter . . . . . . . . . . . . . . . 83

5 Application of the proposed methods on the force distribution of CDPRs $\quad 85$

5.1 Force distribution considering the effect of cable sag . . . . . . . . . . 87

$5.1 .1 \quad$ CDPR description . . . . . . . . . . . . . . . 87

5.1 .2 Kinematic modeling . . . . . . . . . . . . . . 88 
5.1 .3 Force distribution . . . . . . . . . . . . . . . . . . 90

5.2 Determination of the lower-boundary of cable forces . . . . . . . . . 91

5.2.1 Lower force boundary definition . . . . . . . . . . . . . . . . . 91

5.2.2 Pose-dependency . . . . . . . . . . . . . . . . . 92

5.3 A numerical example . . . . . . . . . . . . . . . . . . . . . . . . . . . . . . . . . . . 94

5.3.1 Description of the studied mechanism . . . . . . . . . . . . 94

5.3 .2 Simulation results . . . . . . . . . . . . . . 96

5.3.3 Analysis and discussion . . . . . . . . . . . . . . 99

5.4 Summary of the chapter . . . . . . . . . . . . . . . . . . 100

6 Conclusions and perspectives $\quad 103$

6.1 Conclusions . . . . . . . . . . . . . . . . . 103

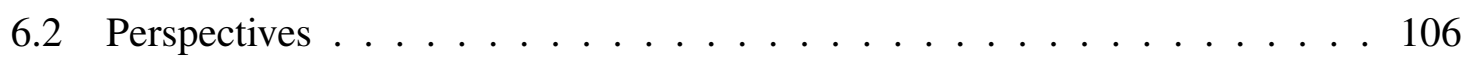

$\begin{array}{lr}\text { References } & 109\end{array}$

$\begin{array}{llr}\text { Appendix A Experimental equipments } & 119\end{array}$ 



\section{List of Figures}

1.1 Two famous rigid-link parallel robots . . . . . . . . . . . . . 2

1.2 Two examples of applications of the rigid-link parallel robots . . . . . . . 2

1.3 Examples of CDPRs with high dynamics . . . . . . . . . . . . . . 3

1.4 Examples of CDPRs with large load capacity $\ldots \ldots \ldots$. . . . . . . 4

1.5 Examples of CDPRs with extremely large scale . . . . . . . . . . 5

1.6 The MARIONET-CRANE for emergency rescue by [Merlet 2010] . . . . 6

1.7 Examples of CDPRs for haptic applications . . . . . . . . . 6

1.8 Example of a suspended and non-redundant actuated CDPR: the CableV $[$ Heyden+ 2006] . . . . . . . . . . . . . . . . 8

1.9 Example of a non-suspended and redundant actuated CDPR: the SEGESTA $[$ Hiller+ 2005; Bruckmann+ 2010] . . . . . . . . . . . 9

2.1 Diagram of a sagging cable . . . . . . . . . . . . . . . . . . 19

2.2 Diagram of a horizontal sagging cable in the cable plan [Starossek 1991b] . 25

2.3 Forces and displacements of an inclined sagging cable . . . . . . . . . 27

2.4 Profiles of the example cable under different external forces: the solid lines and the dashed lines represent the cable profiles and the cable chords, respectively ......................... 31

2.5 Amplitude variation of the trace of the dynamic and static stiffness matrix for the example cable . . . . . . . . . . . . . . . . . . 33

3.1 The schematic diagram of a suspended CDPR . . . . . . . . . . . . 37

3.2 Definition of the static pose error . . . . . . . . . . . . . 41

3.3 The schematic diagram of the studied CDPR (6-DOF suspended by 8 cables) 49

3.4 Static pose error of the end-effector along axis- $z_{G}$ over a sub-workspace $\left(-3 \mathrm{~m} \leqslant x_{G} \leqslant 3 \mathrm{~m},-3 \mathrm{~m} \leqslant y_{G} \leqslant 3 \mathrm{~m}, z_{G}=0.5 \mathrm{~m}\right)$ for $\phi 4 \mathrm{~mm}$ cable and $30 \mathrm{~kg}$ external load . . . . . . . . . . . . . . . 5 50 
3.5 Static pose error of the end-effector along axis- $z_{G}$ over a sub-workspace $\left(-3 \mathrm{~m} \leqslant x_{G} \leqslant 3 \mathrm{~m},-3 \mathrm{~m} \leqslant y_{G} \leqslant 3 \mathrm{~m}, z_{G}=0.5 \mathrm{~m}\right)$ for $\phi 4 \mathrm{~mm}$ cable and $60 \mathrm{~kg}$ external load . . . . . . . . . . . . . . . . . . 50

3.6 Static pose error of the end-effector along axis- $z_{G}$ over a sub-workspace $\left(-3 \mathrm{~m} \leqslant x_{G} \leqslant 3 \mathrm{~m},-3 \mathrm{~m} \leqslant y_{G} \leqslant 3 \mathrm{~m}, z_{G}=0.5 \mathrm{~m}\right)$ for $\phi 8 \mathrm{~mm}$ cable and $30 \mathrm{~kg}$ external load . . . . . . . . . . . . . . . .

3.7 Static pose error of the end-effector along axis- $z_{G}$ over a sub-workspace $\left(-3 \mathrm{~m} \leqslant x_{G} \leqslant 3 \mathrm{~m},-3 \mathrm{~m} \leqslant y_{G} \leqslant 3 \mathrm{~m}, z_{G}=0.5 \mathrm{~m}\right)$ for $\phi 8 \mathrm{~mm}$ cable and $60 \mathrm{~kg}$ external load . . . . . . . . . . . . . . . . .

3.8 Vibration analysis of the studied CDPR when the end-effector is at the center of the sub-workspace $\left(x_{G}=0 \mathrm{~m}, y_{G}=0 \mathrm{~m}\right.$ and $\left.z_{G}=0.5 \mathrm{~m}\right) \ldots \ldots$

3.9 The first natural frequency of the studied CDPR over a sub-workspace $\left(-3 \mathrm{~m} \leqslant x_{G} \leqslant 3 \mathrm{~m},-3 \mathrm{~m} \leqslant y_{G} \leqslant 3 \mathrm{~m}, z_{G}=0.5 \mathrm{~m}\right) \ldots \ldots 55$

4.1 The CDPR prototype with 6 cables . . . . . . . . . . . . . . . . . 59

4.2 The schematic diagram of the CoGiRo [Lamaury+ 2013] . . . . . . . . . 60

4.3 Static experimental setup . . . . . . . . . . . . . . . . . . . . 61

4.4 Effect of external load on the static pose error of the 6-cable CDPR prototype. 62

4.5 Measurement of the static cable forces of the CoGiRo . . . . . . . . . . . . 65

4.6 Modal analysis of the CoGiRo by the shaker . . . . . . . . . . . 67

4.7 Experimental and simulation results of the vibration analysis of the $5^{\text {th }}$ cable at the pose near the center of the workspace $(x=0.012 \mathrm{~m} ; y=0.0697 \mathrm{~m}$; $\left.z=1.219 \mathrm{~m} ; \alpha, \beta, \gamma=0^{\circ}\right) \ldots \ldots \ldots \ldots$

4.8 Experimental and simulation results of the vibration analysis of the $5^{\text {th }}$ cable at the pose near the edge of the workspace $(x=4.012 \mathrm{~m} ; y=0.0697 \mathrm{~m}$; $\left.z=1.219 \mathrm{~m} ; \alpha, \beta, \gamma=0^{\circ}\right)$

4.9 Experimental and simulation results of the vibration analysis of the endeffector at the pose near the center of the workspace $(x=0.012 \mathrm{~m} ; y=0.0697$ $\left.\mathrm{m} ; z=1.219 \mathrm{~m} ; \alpha, \beta, \gamma=0^{\circ}\right) \ldots \ldots \ldots \ldots$

4.10 Experimental and simulation results of the vibration analysis of the endeffector at the pose near the edge of the workspace $(x=4.012 \mathrm{~m} ; y=0.0697$ $\left.\mathrm{m} ; z=1.219 \mathrm{~m} ; \alpha, \beta, \gamma=0^{\circ}\right) \ldots \ldots \ldots 72$

4.11 Free vibration experiment at an emergency stop . . . . . . . . . . . . . . 76

4.12 Experimental results of the free vibration analysis of the $5^{\text {th }}$ cable during a trajectory containing an emergency stop . . . . . . . . . . . . . . 
4.13 Experimental results of the free vibration analysis of the end-effector along axis- $x$ during a trajectory containing an emergency stop . . . . . . . . 78

4.14 Experimental results of the free vibration analysis of the end-effector along axis- $z$ during a trajectory containing an emergency stop . . . . . . . . . . 79

4.15 Experimental setup of the analysis along a trajectory . . . . . . . . . 80

4.16 Pose error of the end-effector during the trajectory from $x=1 \mathrm{~m} ; y=-2 \mathrm{~m}$; $z=0 \mathrm{~m} ; \alpha, \beta, \gamma=0^{\circ}$ to $x=-0.5 \mathrm{~m} ; y=-0.7 \mathrm{~m} ; z=-0.25 \mathrm{~m} ; \alpha, \beta, \gamma=$ $0^{\circ}$ with different trajectory time durations . . . . . . . . . .

4.17 Experimental results of the vibration analysis of the end-effector along axis- $z$ during the trajectory of 30 seconds $\ldots \ldots \ldots 82$

5.1 Kinematic model of a general CDPR considering cable sag . . . . . . . . 88

5.2 Flow chart of the force distribution of CDPRs . . . . . . . . . . . . . 93

5.3 Configuration of the 6 DOF CDPR driven by 8 cables . . . . . . . . . . . 95

5.4 The variation of the lower-boundary along the trajectory: the blue solid lines represent the results of the fixed lower-boundary; the red dash lines represent the results of the pose-dependent lower-boundary . . . . . . . . . . . 97

5.5 The variation of the cable force along the trajectory: the blue solid lines represent the results of the fixed lower-boundary; the red dash lines represent the results of the pose-dependent lower-boundary . . . . . . . . . . . . 98

5.6 The energy consumption of the CDPR along the trajectory: the blue solid lines represent the results of the fixed lower-boundary; the red dash lines represent the results of the pose-dependent lower-boundary . . . . . . . .

A.1 The electrodynamic vibration shaker . . . . . . . . . . . . . . . 119

A.2 The Nikon K-600 camera system . . . . . . . . . . . . . . . . . . 120

A.3 Sensors in the experiments . . . . . . . . . . . . . . . 120

A.4 The data acquisition system . . . . . . . . . . . . . . . 121 



\section{List of Tables}

2.1 Physical parameters of the studied cable . . . . . . . . . . . . 30

2.2 Static cable parameters of the example cable under different external forces 30

2.3 Static compliance and stiffness matrix of the example cable under different external forces . . . . . . . . . . . . . . . . 31

2.4 Dynamic cable parameters of the example cable under different external forces 32

3.1 Configuration parameters of the studied CDPR: coordinates of $B_{i}$ expressed in global frame $\mathfrak{R}_{G}$; coordinates of $A_{i}$ expressed in end-effector frame $\mathfrak{R}_{e}$.

3.2 Average values of the pose errors in the sub-workspace $\left(-3 \mathrm{~m} \leqslant x_{G} \leqslant 3 \mathrm{~m}\right.$, $\left.-3 \mathrm{~m} \leqslant y_{G} \leqslant 3 \mathrm{~m}, z_{G}=0.5 \mathrm{~m}\right) \ldots \ldots \ldots . \ldots 50$

4.1 Configuration parameters of the 6-cable CDPR prototype: coordinates of the points $B_{i}$ in the global frame $\Re_{G}$ and that of $A_{i}$ in the local frame $\mathfrak{R}_{e} \ldots 59$

4.2 Cable parameters of the 6 -cable CDPR prototype . . . . . . . . . . 59

4.3 Configuration parameters of the CoGiRo: coordinates of $B_{i}$ expressed in global frame $\mathfrak{R}_{G}$; coordinates of $A_{i}$ expressed in end-effector frame $\mathfrak{R}_{e} \ldots 61$

4.4 Static forces of the driving cables measured at different poses of the endeffector in the workspace . . . . . . . . . . . . . 66

4.5 Cable natural frequencies identified by experiments and simulations at the pose near the center of the workspace $(x=0.012 \mathrm{~m} ; y=0.0697 \mathrm{~m} ; z=1.219$ $\left.\mathrm{m} ; \alpha, \beta, \gamma=0^{\circ}\right) \ldots \ldots \ldots \ldots \ldots \ldots$

4.6 Cable natural frequencies identified by experiments and simulations at the pose near the edge of the workspace $(x=4.012 \mathrm{~m} ; y=0.0697 \mathrm{~m} ; z=1.219$

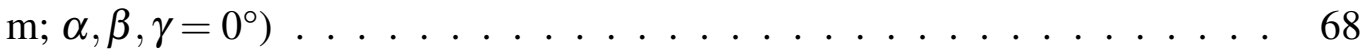

5.1 Parameters of the end-effector and the driving cables . . . . . . . . . 95

5.2 Coordinates of the attachments $B_{i}$ in the global frame and $A_{i}$ in the local end-effector frame . . . . . . . . . . . . . . . . . . 96 
5.3 Simulation results of the force distribution $\ldots \ldots \ldots$. . . . . . 100 


\title{
Nomenclature
}

\author{
Abbreviation \\ CDPR Cable-Driven Parallel Robot, page 1 \\ DSM Dynamic Stiffness Matrix, page 14 \\ FEM Finite Element Method, page 14 \\ FRF $\quad$ Frequency Response Function, page 64
}

\section{Coordinate system}

$\Re_{e} \quad$ Local frame fixed on the end-effector, page 37

$\Re_{G} \quad$ Global frame of CDPRs, page 37

$\Re_{B_{i}} \quad$ Auxiliary frame, page 37

$\mathfrak{R}_{c i} \quad$ Local cable frame, page 37

\section{Matrix}

${ }^{e} \mathbf{K}_{E}(\omega) \quad$ Dynamic stiffness matrix of CDPRs expressed in the end-effector frame $\mathfrak{R}_{e}$, page 44

${ }^{G} \mathbf{K}_{E}(\omega)$ Dynamic stiffness matrix of CDPRs expressed in the global frame $\Re_{G}$, page 44

${ }^{G} \mathbf{K}_{i}(\omega) \quad$ Dynamic stiffness matrix of the $i^{t h}$ inclined sagging cable expressed in the global frame $\Re_{G}$, page 44

${ }^{G} \mathbf{T}_{e} \quad$ Rotation matrix transferring the coordinates in $\Re_{e}$ to their corresponding coordinates in $\Re_{G}$, page 38 
${ }^{G} \mathbf{T}_{c i} \quad$ Rotation matrix that transfers the coordinates in $\mathfrak{R}_{c i}$ to their corresponding coordinates in $\mathfrak{R}_{G}$, page 38

${ }^{c i} \mathbf{K}_{i}(\omega) \quad$ Dynamic stiffness matrix of the $i^{t h}$ inclined sagging cable expressed in the cable frame $\mathfrak{R}_{c i}$, page 44

$\mathbf{C}_{\text {axial }}^{2 \mathrm{D}}$

$\mathbf{C}_{\text {sag }}^{2 \mathrm{D}}$

$\mathbf{C}_{\text {static }}^{2 \mathrm{D}}$

$\mathbf{J}^{T}$

$\mathbf{K}_{\text {dyn-h }}^{3 \mathrm{D}}(\omega)$

$\mathbf{K}_{\text {static }}^{2 \mathrm{D}}$

$\mathbf{H}(\omega)$

$\mathbf{K}_{\text {dyn-h }}^{2 \mathrm{D}}(\omega)$

$\mathbf{K}_{\mathrm{dyn}}^{2 \mathrm{D}}(\omega)$

$\mathbf{K}_{\mathrm{dyn}}^{3 \mathrm{D}}(\omega)$

${ }^{e} \mathbf{M}$

\section{Scalar}

$\alpha$

$\Delta l$

$\dot{q}_{i}$

$\rho$

$\lambda^{2} \quad$ Fundamental cable parameter representing the elastic stiffness relative to the

Compliance matrix caused by the axial cable elasticity, page 24

Compliance matrix caused by the sag-introduced flexibility, page 24

Static compliance matrix of an inclined sagging cable ( 2 by 2 matrix), page 23

Transposition of the Jacobian matrix, page 39

Dynamic stiffness matrix of a horizontal sagging cable in 3-D, page 27

Static stiffness matrix of an inclined sagging cable ( 2 by 2 matrix), page 23

Frequency Response Function matrix, page 47

Dynamic stiffness matrix of a horizontal sagging cable in the cable plan (2-D), page 26

Dynamic stiffness matrix of an inclined sagging cable in the cable plan, page 28

Dynamic stiffness matrix of an inclined sagging cable in 3-D, page 29

6 by 6 mass matrix of the end-effector expressed in frame $\Re_{e}$, page 45

\section{Inclined angle of the cable, page 21}

$l \quad$ Cable elongation, page 23

Time derivatives of the generalized coordinate, page 45 catenary stiffness, page 27

Cable mass per unit length, page 21 
$\tau$

$\varepsilon$

A

$d$

E

$F_{i}$

$l_{c}$

$L_{e}$

$l_{s}$

$l_{u s}$

$m$

n

$q_{i}$

$s$

$T$

V

$\Omega_{c}$

$\Omega$

\section{Vector}

$\overline{\mathbf{f}}$

$\overline{\mathbf{q}}$

Static cable tension at the section where the cable is parallel to the chord, page 21

Ratio between horizontal cable weight and cable tension, page 27

Unstrained cross-sectional area of the cable, page 21

Cable sag perpendicular to the chord, page 21

Young's modulus, page 21

Nonconservative generalized force or moment applied to the end-effector, page 45

Chord length of the cable, page 21

Cable length parameter, page 27

Strained cable length between points $C$ and $B$, page 21

Unstrained cable length between points $C$ and $B$, page 21

Number of driving cables, page 39

Number of DOFs of the end-effector, page 39

Generalized coordinate, page 45

Lagrangian coordinate in the unstrained cable profile, page 21

Kinetic energy of the system, page 45

Potential energy of the system, page 45

Dimensionless frequency-damping parameter, page 27

Dimensionless frequency parameter, page 27

Column complex-valued vector representing the amplitudes and the initial phases of the excitations, page 47

Column complex-valued vector representing the amplitudes and the initial phases of the responses, page 47 
$\dot{\mathbf{q}}$ Column vectors of the time derivatives of the generalized coordinates, page 45

${ }^{G} \mathbf{f}_{A i} \quad$ Cable force vector at point $A_{i}$ expressed in global frame $\mathfrak{R}_{G}$, page 39

${ }^{G} \mathbf{f}_{e x} \quad$ External force vector expressed in global frame $\mathfrak{R}_{G}$, page 39

${ }^{G} \mathbf{m}_{e x} \quad$ External moment vector expressed in global frame $\mathfrak{R}_{G}$, page 39

${ }^{c i} \mathbf{f}_{A i} \quad$ Cable force vector at point $A_{i}$ expressed in global frame $\mathfrak{R}_{c i}$, page 39

$\mathbf{f}(t) \quad$ Column vector of the harmonic excitations applied to the end-effector, page 47

$\mathbf{q}(t) \quad$ Column vector of the harmonic responses of the end-effector, page 47

$\mathbf{f}_{\text {ideal }} \quad$ Column vector of the ideal cable forces, page 40

q Column vectors of the generalized coordinates, page 45

w Column vector of the nonconservative forces and moments, page 45 


\section{Chapter 1}

\section{Introduction}

\section{Contents}

1.1 Presentation of CDPRs $\ldots \ldots \ldots \ldots \ldots \ldots$

1.2 Problematic and current researches $\ldots \ldots \ldots \ldots \ldots$

1.3 Objectives of this thesis $\ldots \ldots \ldots \ldots \ldots$

\subsection{Presentation of CDPRs}

A generalized parallel robot can be defined as a closed-loop kinematic chain mechanism whose end-effector is linked to the base by several independent kinematic chains [Merlet 2006].

Traditional parallel robots use rigid links to form the kinematic chains, such as the famous Gough-Stewart platform (fig. 1.1a) [Gough 1957; Stewart 1965] and the well known Delta robot (fig. 1.1b) [Clavel 1991]. Rigid-link parallel robots are used in various applications, such as tire test machines (fig. 1.1a) [Gough 1957], flight simulators (fig. 1.2a) [Koevermans+ 1975], surgery operations (fig. 1.2b) [Briot+ 2007], space antennas [Dunlop+ 1999], machine tools [Weck+ 2002], etc. Compared to their serial counterparts, rigid-link parallel robots have advantages such as large load carrying capacity, high stiffness, low inertia, etc. However, their main inconvenience is the relatively small workspace.

Cable-driven parallel robots (CDPRs) are a special variant of traditional rigid-link parallel robots. They use flexible cables instead of rigid links to connect the movable end-effector and the fixed base. The end-effector is manipulated by changing the length of the cables that are actuated by fixed motors and winches. As a kind of parallel robots, CDPRs also have 
advantages in load capacity, stiffness, energy efficiency and so on. Furthermore, CDPRs overcome the major weakness of rigid-link parallel robots: workspace. Unlike rigid links, cable lengths can vary in a wide range, which enlarges the workspace of CDPRs. These characteristics have attracted a lot of interest of researchers in the past few decades.

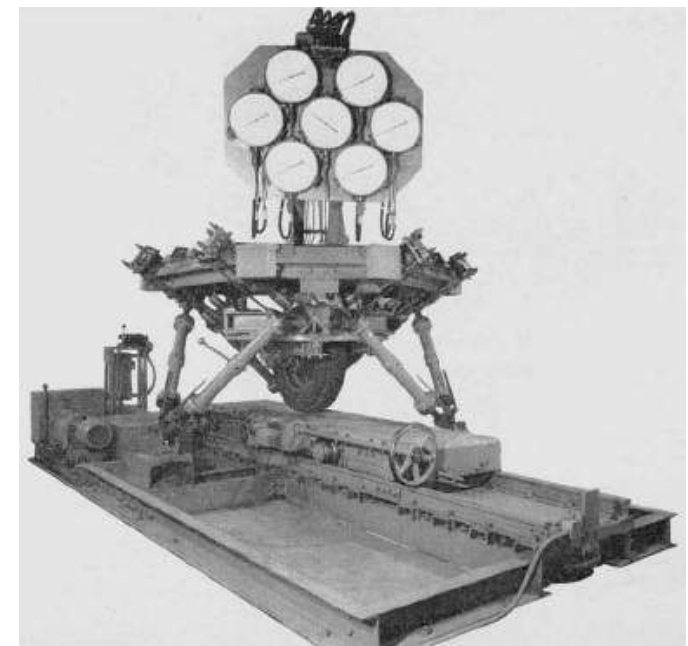

(a) The original Gough-Stewart platform [Gough 1957]

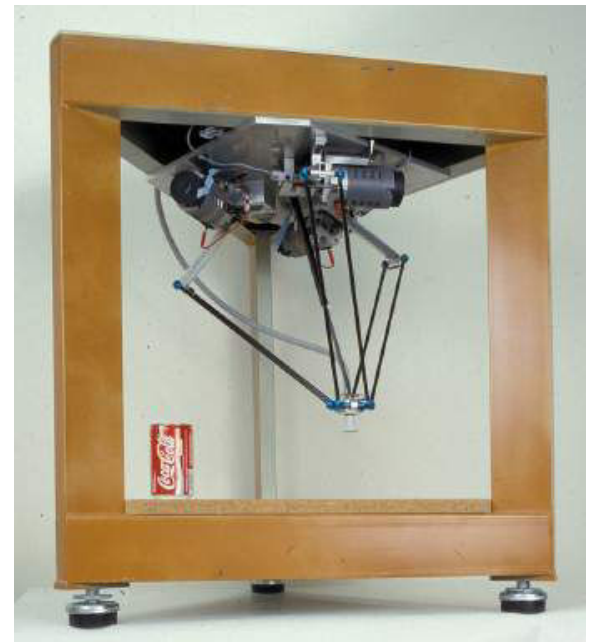

(b) The first Delta robot [Clavel 1991]

Fig. 1.1 Two famous rigid-link parallel robots

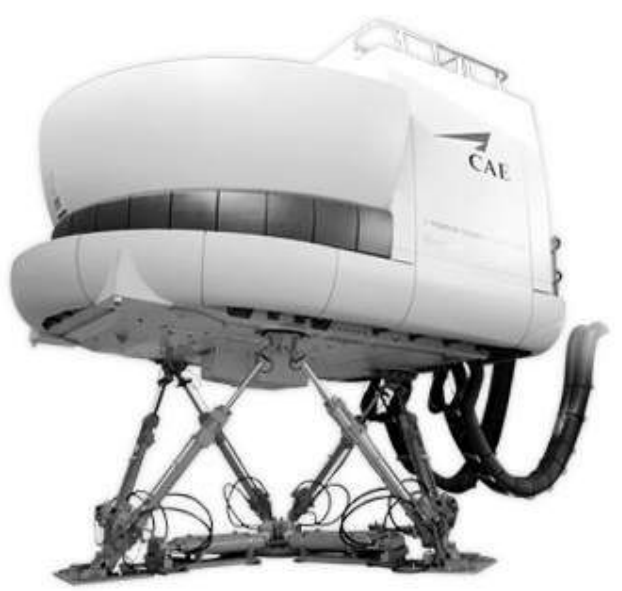

(a) The Airbus A320 simulator ${ }^{1}$

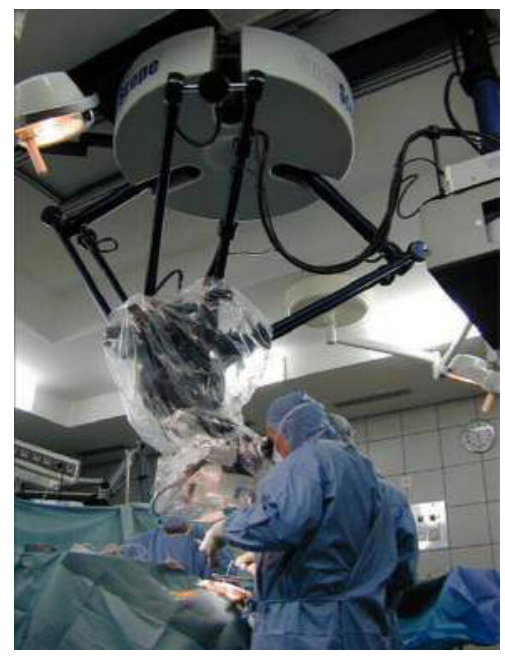

(b) The Surgiscope ${ }^{2}$

Fig. 1.2 Two examples of applications of the rigid-link parallel robots

\footnotetext{
${ }^{1}$ The Airbus A320 simulator is a product of Baltic Aviation Academy: http://www.balticaa.com

${ }^{2}$ The Surgiscope is a product of ISIS corporation: http://www.isis-robotics.com/
} 


\subsubsection{Advantages and applications of CDPRs}
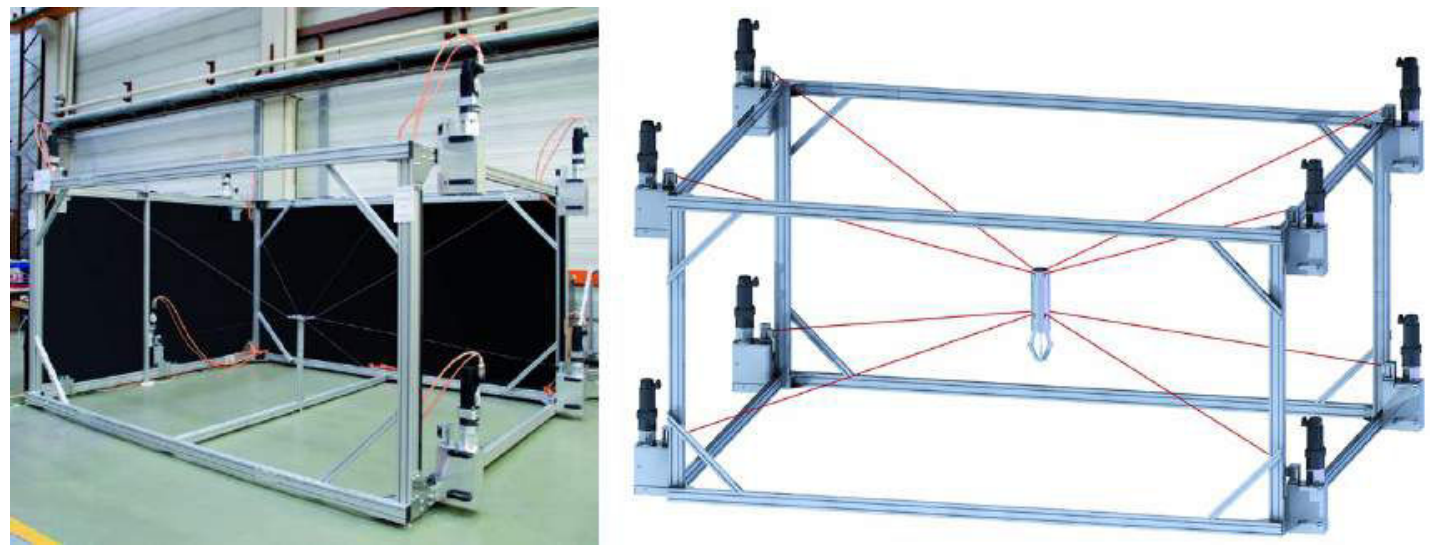

(a) The IPAnema [Pott+ 2013]
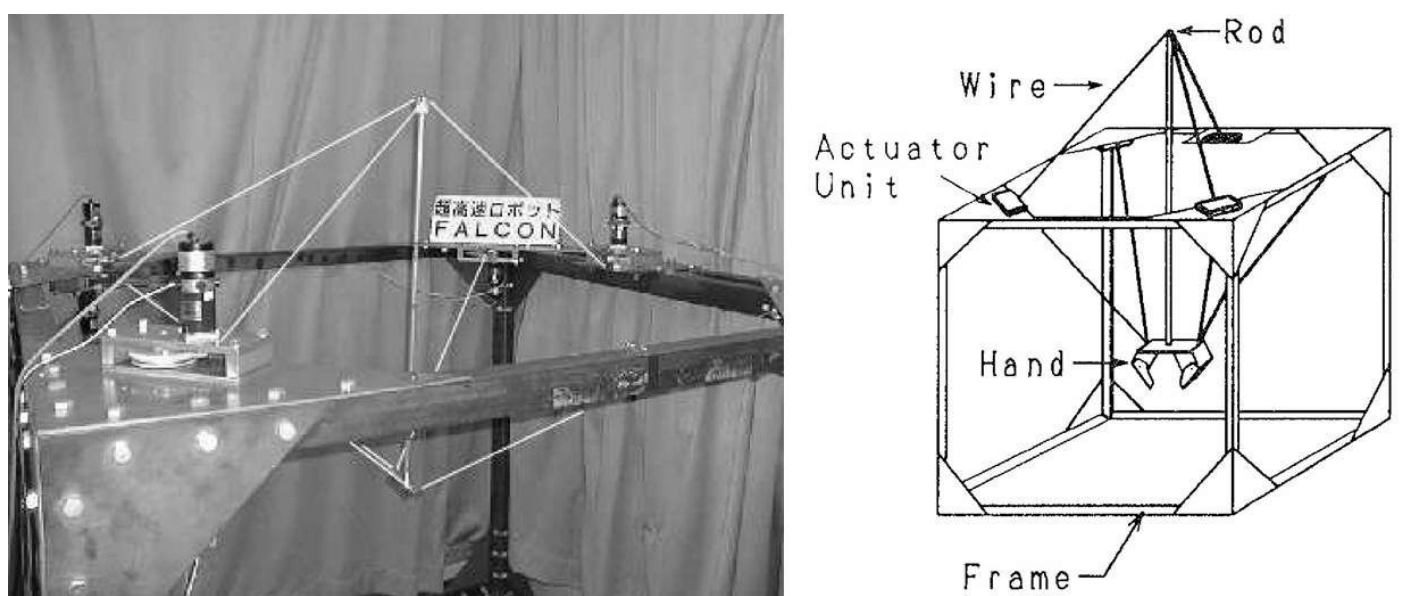

(b) The FALCON [Kawamura+ 1995]

Fig. 1.3 Examples of CDPRs with high dynamics

\section{Large workspace}

Firstly of all, CDPRs can achieve large workspaces. As cables are flexible, they can be easily released and retracted through winches. Thus, cables allow a much larger range of motion compared to conventional rigid links. For example, the workspace of the prototype REELAX8-S [Lamaury 2013], a 6-DOF CDPR suspended by 8 cables, can reach up to 78\% of the volume of the robot. By contrast, the workspace of a rigid-link parallel robot PAR4 [Lamaury 2013; Nabat 2007] represents only 31\% of the volume of the robot. 


\section{High dynamics}

Furthermore, CDPRs have high dynamics. Because cables are lighter than most of the conventional rigid links and they have small mobile mass and low inertia, CDPRs are suitable for high velocity and/or high acceleration applications, such as the IPAnema (fig. 1.3a) [Pott+ 2010; Pott+ 2013] and the FALCON (fig. 1.3b) [Kawamura+ 1995; Kawamura+ 2000]. For example the FALCON robot can attain a peak speed of about $13 \mathrm{~m} / \mathrm{s}$, and a peak acceleration of $43 \mathrm{G}$ [Kawamura+ 1995].

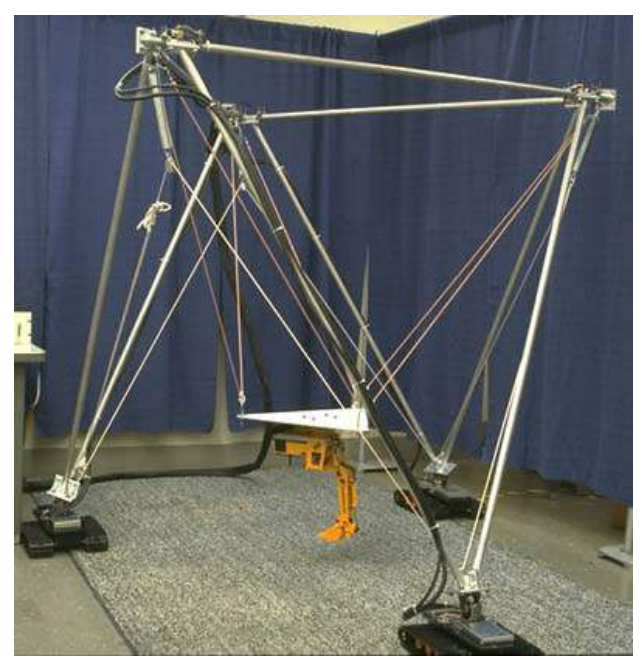

(a) The ROBOCRANE [Albus+ 1993]

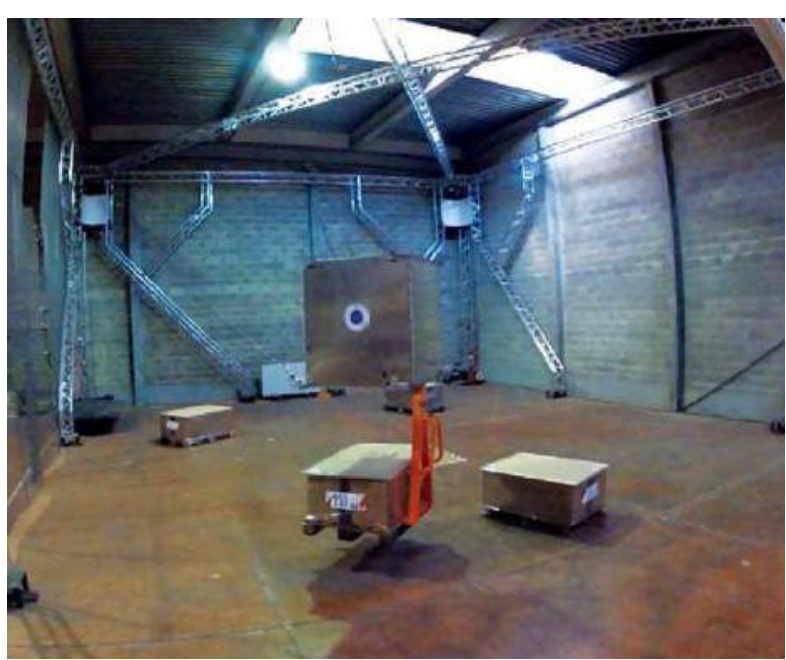

(b) The CoGiRo [Lamaury 2013]

Fig. 1.4 Examples of CDPRs with large load capacity

\section{Large payload capacity and high energy efficiency}

In addition, CDPRs have large payload capacity and high energy efficiency. For serial robots, the energy consumption is high and the payload capacity is low, because each actuator has to carry not only the payload in the end-effector, but also the weight of all the subsequent links and actuators. Traditional rigid-link parallel robots are more efficient compared to serial robots, because their actuators are usually fixed and the payload can be shared by each link. But the movement of the rigid links still consume lots of useless energy. CDPRs use lightweight cables instead of relatively heavy rigid links. They usually have stationary heavy components and few moving parts. The energy consumption is focused on the movement of the end-effector and payload is shared by many driving cables, resulting very high energy efficiency and high payload-to-weight ratios, such as the ROBOCRANE (fig. 1.4a) [Albus+ 1992; Albus+ 1993; Bostelman+ 1994] and the CoGiRo (fig. 1.4b) [Lamaury+ 2013]. For 
example the load capacity of the CoGiRo prototype [Lamaury 2013] can reach $500 \mathrm{~kg}$, while the total mass of the moving parts of the robot including the end-effector and the driving cables is only about $100 \mathrm{~kg}$.

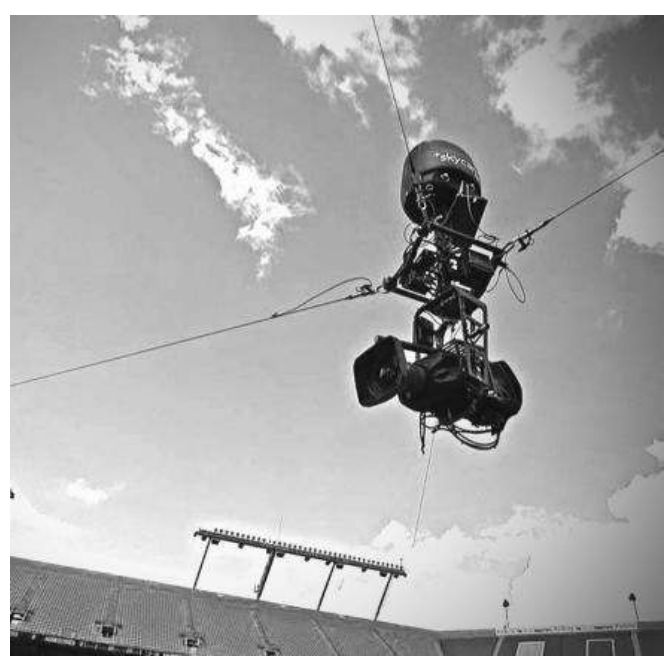

(a) The Skycam ${ }^{3}$

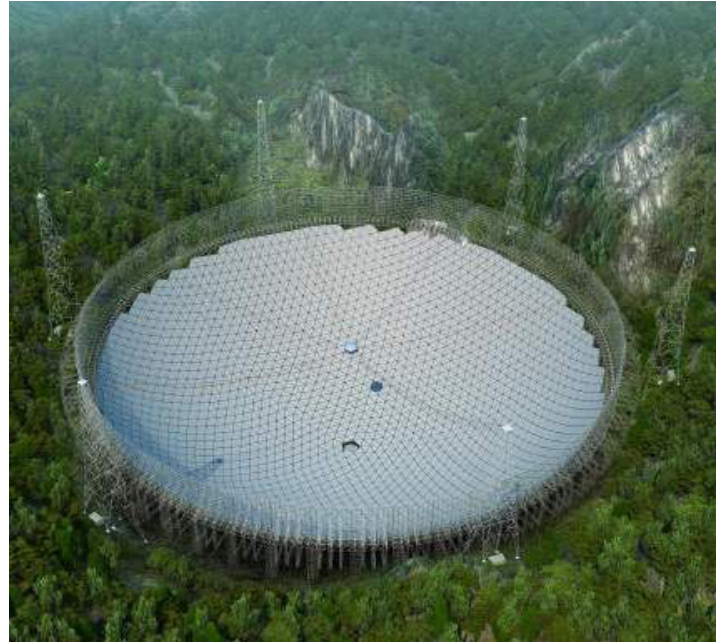

(b) The FAST [Nan 2006]

Fig. 1.5 Examples of CDPRs with extremely large scale

\section{Low cost}

Besides the above advantages, CDPRs have low cost. As the price of electronic components are becoming lower, while the price of mechanical parts keeps quite stable, there is a trend to design robots with simple mechanical structures but relatively complex electronic devices. CDPRs fit this trend and thus they can be designed in extremely large scale within an acceptable cost, such as the Skycam ${ }^{3}$ (fig. 1.5a), and the Five hundred meter Aperture Spherical Telescope (FAST, fig. 1.5b) [Zi+ 2008; Nan 2006].

\section{Simple structure}

Another advantage of CDPRs is their simple structure. They can be easily disassembled, reassembled, transported and reconfigured. The position of the attachment points can be modified and determined by a calibration of the system [Sandretto+ 2013a; Borgstrom+ 2009; Miermeister+ 2012]. This characteristic makes CDPRs suitable for search and rescue applications (fig. 1.6) [Tadokoro+ 1999; Bosscher+ 2005; Merlet+ 2010; Merlet 2010].

\footnotetext{
${ }^{3}$ Skycam is a product of Skycam company: http://www.skycam.tv/
} 


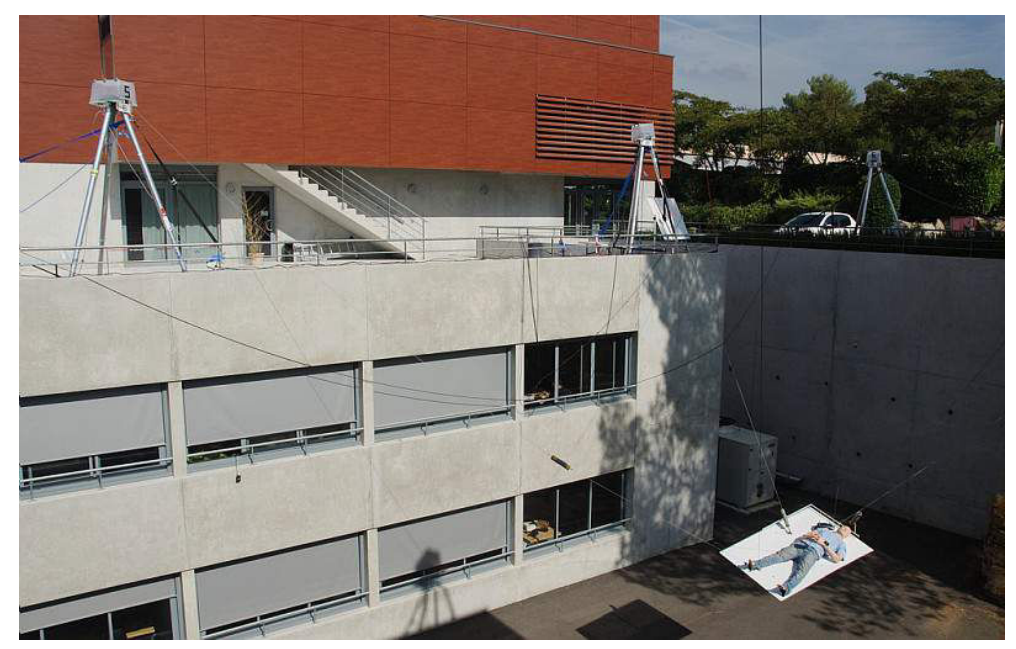

Fig. 1.6 The MARIONET-CRANE for emergency rescue by [Merlet 2010]

\section{Good safety}

Last but not least, CDPRs have a good safety during operations. As cables are flexible, they provide a natural protection during interference with each other or with other objects in the environment [Mao+ 2012; Rosati+ 2005]. This characteristic makes CDPRs quite useful for haptic devices, such as the NEREBOT (fig. 1.7a) [Gallina+ 2002] and the STING-MAN (fig. 1.7b) [Surdilovic+ 2007].

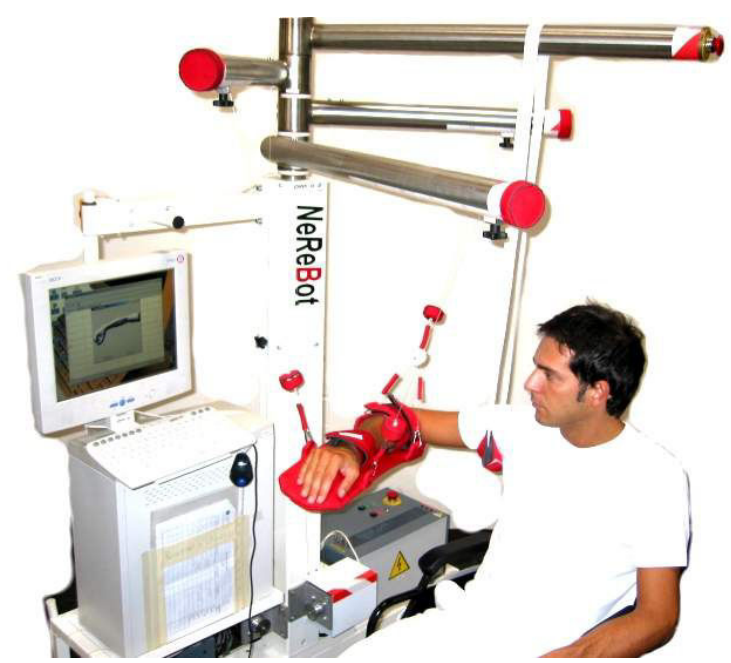

(a) The NEREBOT [Gallina+ 2002]

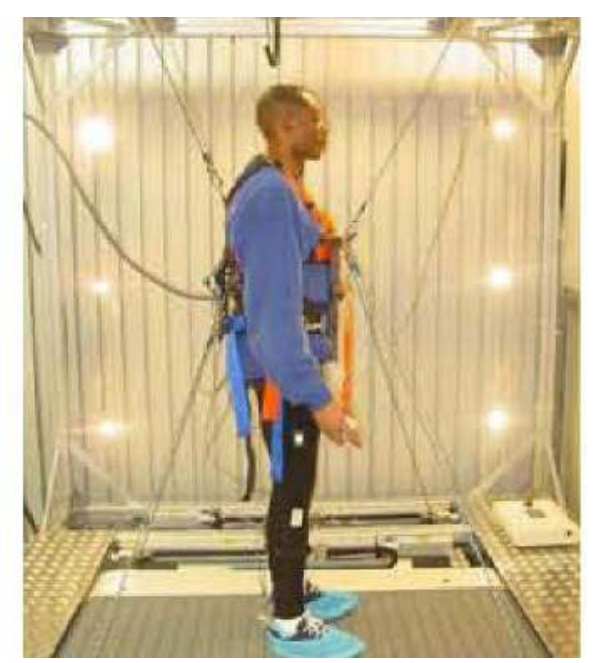

(b) The STING-MAN [Surdilovic+ 2007]

Fig. 1.7 Examples of CDPRs for haptic applications 


\subsubsection{Classification of CDPRs}

\section{Suspended and non-suspended CDPRs}

According to the arrangement of cables, two kinds of CDPRs can be considered. One kind is suspended CDPRs, where all the driving cables are above the end-effector and gravity acts as a virtual cable to keep equilibrium, such as the CableV (fig. 1.8) [Heyden+ 2006], the ROBOCRANE (fig. 1.4a) [Albus+ 1993], the CoGiRo (fig. 1.4b) [Lamaury 2013], the Skycam (fig. 1.5a), the FAST (fig. 1.5b) [Nan 2006], etc. The other kind is non-suspended CDPRs, where at least one driven cable is below the end-effector, such as the FALCON (fig. 1.3b) [Kawamura+ 1995], the IPAnema (fig. 1.3a) [Pott+ 2013], the SEGESTA (fig. 1.9) [Hiller+ 2005; Fang+ 2004], etc. It should be noticed that CDPRs working on a horizontal plane are classified as non-suspended CDPRs. Because the weight of the end-effector is balanced by the support of the plane, gravity has no effect on the equilibrium of the end-effector.

For suspended CDPRs, since all the cables are above the end-effector, the payload can be shared by each cable, thus suspended CDPRs usually have big load capacity. Moreover, compared with non-suspended CDPRs, there is less possibility for the cables to interfere with other objects in the environment because no cable is lower than the end-effector. These two characteristics make suspended CDPRs quite suitable for pick-and-place applications, just like cranes. However, suspended CDPRs may become unstable, easy to vibrate and even out of control under external disturbances, especially when the end-effector is unloaded. This weakness is due to the low stiffness of CDPRs along the vertical direction. Therefore, it is necessary to pay more attention to the stiffness analysis of suspended CDPRs in order to improve their accuracy and reduce their vibration.

For non-suspended CDPRs, stiffness and positioning accuracy can be improved and vibration can be reduced through increasing the internal cable forces. Consequently, nonsuspended CDPRs usually have better performances in high velocity and/or acceleration applications compared to suspended CDPRs. However, the motor power and energy consumption will significantly increase with growing internal cable forces, which can augment the fabrication and the operation costs.

\section{Redundant actuated and non-redundant actuated CDPRs}

According to the relationship between the number of driving cables $(m)$ and the number of degree of freedom of the end-effector $(n)$, two kinds of CDPRs can be discussed. One kind is redundant actuated CDPRs, where $m>n$, such as the FALCON ( $m=7, n=6$, fig. 1.3b) 


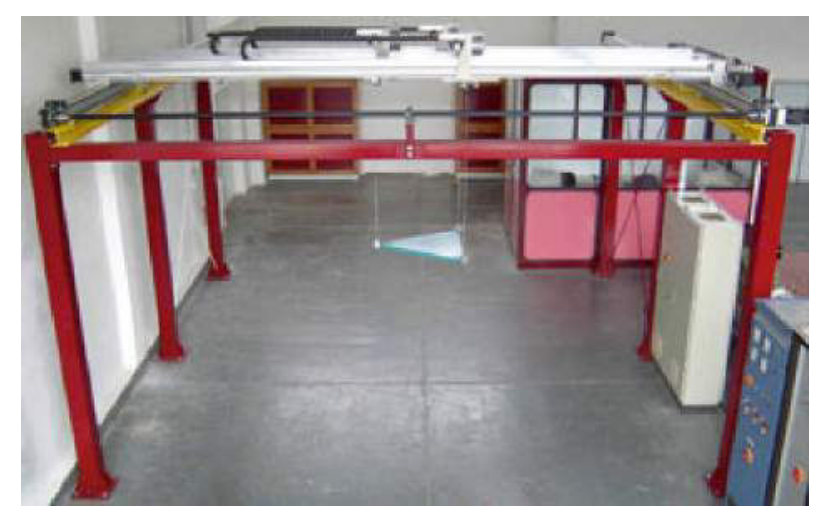

(a) The prototype

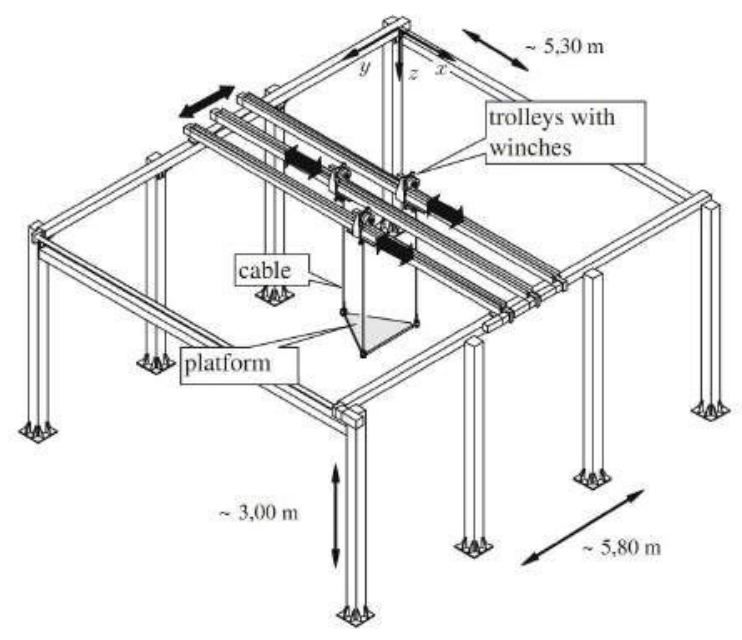

(b) The schematic diagram

Fig. 1.8 Example of a suspended and non-redundant actuated CDPR: the CableV [Heyden+ 2006]

[Kawamura+ 1995], the CoGiRo ( $m=8, n=6$, fig. 1.4b) [Lamaury 2013], the IPAnema ( $m=8$, $n=6$, fig. 1.3a) [Pott+ 2013], the SEGESTA ( $m=8$ or $7, n=6$, fig. 1.9) [Hiller+ 2005], and the Skycam ( $m=4, n=3$, fig. 1.5a). The other kind is non-redundant actuated CDPRs, where $m \leq n$, such as the CableV ( $m=3, n=3$, fig. 1.8) [Heyden+ 2006], the ROBOCRANE ( $m=6$, $n=6$, fig. 1.4a) [Albus+ 1993], and the FAST ( $m=6, n=6$, fig. 1.5b) [Nan 2006].

Compared to non-redundant actuated CDPRs, redundant actuated CDPRs have some advantages. Through adding redundant driving cables, workspace can be extended, singularity can be reduced and stiffness can be increased. In addition, payload are redistributed to more cables and the tension of the driving cable can be decreased, which can decrease the motor size. Moreover, safety can be enhanced by using redundant cables. The end-effector maybe still controllable even if some of the redundant cables are out of control. Another 


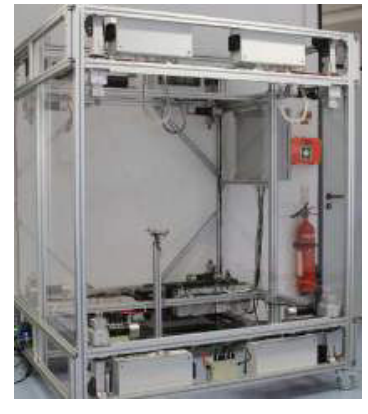

(a) The prototype

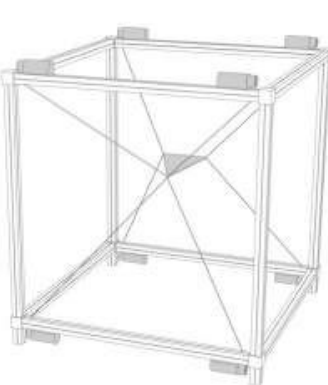

(b) The schematic diagram (8 or 7 cables)

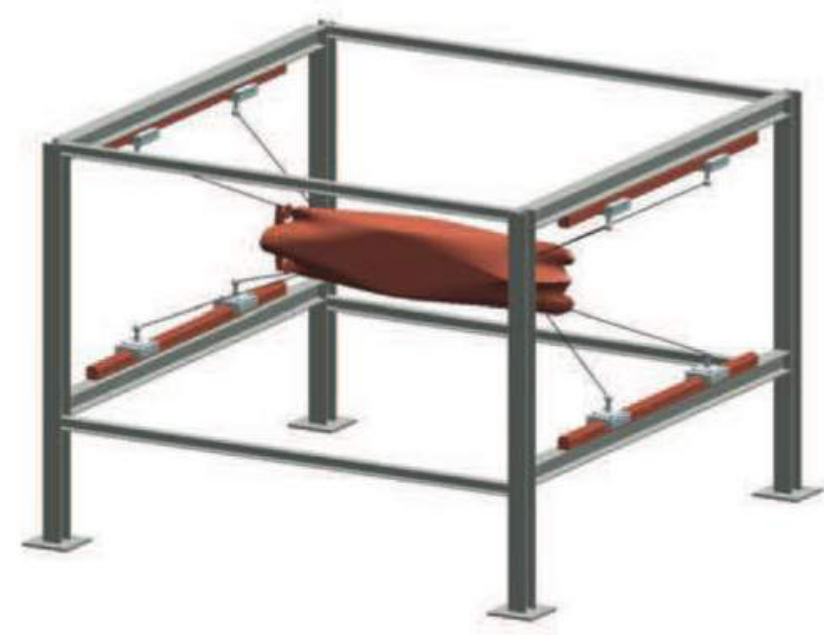

(c) Its application in wind tunnels

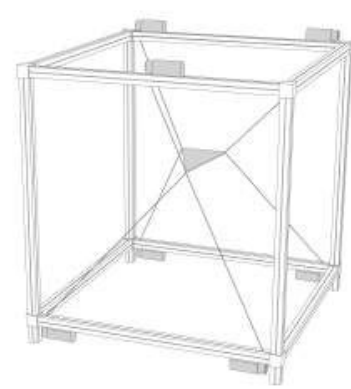

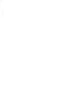


In order to guarantee the positive cable forces, lots of works are presented in literature aiming to find optimal and positive distributions of cable forces, especially for high redundant actuated CDPRs [Gosselin+ 2011; Mikelsons+ 2008; Pott+ 2009; Khosravi+ 2013]. A related problem is the controller design of CDPRs, which must take the positive-cable-force requirement into account. A number of researches have investigated the controller design of CDPRs [Oh+ 2005; Fang+ 2004; Vafaei+ 2011; Zi+ 2008; Dallej+ 2012; Lamaury+ 2013]. The force distribution problem of CDPRs will be further addressed in Chapter 5.

Another issue is the determination of the workspace. The workspace of CDPRs is not only related to the geometric constrain of CDPRs, but also limited by the cable tensions. Considering the positive-cable-force requirement and the static and/or dynamic performances of CDPRs, different kinds of workspace can be defined, such as the Static Equilibrium Workspace [Pusey+ 2004], the Dynamic Workspace [Barrette+ 2005], the Wrench-Feasible Workspace [Bosscher+ 2006; Gouttefarde+ 2007], etc.

\subsubsection{Stiffness analysis}

Due to the compliance of cables, the stiffness of robots becomes a vital concern [Gouttefarde+ 2012; Riehl+ 2009], especially for suspended CDPRs. Stiffness has a significant effect on the static and dynamic behaviors of CDPRs, such as kinematics, positioning accuracy, force distribution, vibration and control [Gosselin 1990; Merlet 2006]. Deficient static stiffness can decrease the positioning accuracy of CDPRs, and bad dynamic stiffness characteristics can lead to vibration and long settling time. Although stiffness has been well studied in the last few decades for rigid-link parallel robots [Gosselin 1990; Carbone 2011; Courteille+ 2009; Merlet 2006; Deblaise+ 2006; ElKhasawneh+ 1999], there is little literature on the stiffness problem of CDPRs. This thesis will focus on the static and dynamic stiffness analyses of CDPRs.

\section{Static stiffness}

An important issue closely associated with the static stiffness is the positioning accuracy of CDPRs. For example, this issue is especially important for suspended pick-and-place CDPRs, such as the CoGiRo [Nguyen+ 2013]. The reasons are as following. On one hand, cables should be designed strong enough to sustain the weight of heavy cargo. A common way to enhance the load capacity of cables is to increase their diameter, which directly leads significant growth of cable weight. One the other hand, the end-effector should be designed as light as possible to improve the efficiency of pick-and-place. When the end-effector is 
unloaded, the cable sag will become quite significant. In this situation, the ideal cable model and the spring cable model that neglect the effect of cable weight on the static cable profile can bring big error in the prediction of the pose of the end-effector. For a traditional rigid-link manipulator, the pose error can be defined by its Cartesian stiffness matrix, assuming the compliant displacements of the end-effector are small [Carbone 2011]. However, due to the nonlinearity of sagging cable, the small displacement assumption is not valid. For example, the static compliant displacement of the CoGiRo $(15 \times 11 \times 6 \mathrm{~m}, l \times w \times h)$ can even research $13.9 \mathrm{~cm}$ with $210 \mathrm{~kg}$ external load according to [Nguyen+ 2013]. In this situation, other methods should be found to compute the pose error.

The compliance of driving cables is the major factor that affects the positioning accuracy of CDPRs. Considering the physical cable characteristics, the compliance of cables mainly has two sources. One is the axial stiffness of the cables, which is associated with the elastic modulus of the material of cables. The other is the sag-introduced flexibility, which corresponds to the gravitational potential energy stored in the cable. Therefore, for the stiffness and accuracy analysis of CDPRs, an important issue is cable modeling. Many studies used linear or non-linear spring as cable model [Verhoeven+ 1998; Dagalakis+ 1989; Kawamura+ 1995; Kawamura+ 2000; Behzadipour+ 2006; Korayem+ 2007; Bedoustani+ 2008; Vafaei+ 2011; Khosravi+ 2013]. This approach only considers the elasticity along cable axis and assumes cable as massless spring. This assumption is not accurate enough, especially for CDPRs with heavy and/or long-span cables. In fact, the static cable profile under the effect of gravity is not a straight line but a sagging curve. Thus the axial cable elasticity is not the only source of the static stiffness of CDPRs, since sag-introduced flexibility should also be considered. A well known model considering the sag-introduced stiffness is the static sagging cable model deriving from civil engineering [Irvine 1992]. It is used in several previous researches [Kozak+ 2006; Riehl+ 2009; Gouttefarde+ 2012; Sandretto+ 2013b; Arsenault 2013]. The sagging cable model considers the cable elasticity and the effect of cable weight on the static cable profile. It is more accurate than the spring cable model in the static stiffness analysis of CDPRs. In previous researches [Kozak+ 2006; Riehl+ 2009; Arsenault 2013], the effect of cable sag on the static stiffness of CDPRs is only verified by numerical simulations. Experimental verification of the static stiffness is performed on single cables, but not on CDPRs in [Kozak+ 2006; Irvine 1992]. To our best knowledge, the only experimental validation of the sagging cable model on a complete CDPR is presented in [Nguyen+ 2013].

Since the stiffness performances of robots are important for the design and control of CDPRs, another considerable issue is the index of stiffness performance evaluation. 
Most studies [Arsenault 2013; Verhoeven+ 1998; Dagalakis+ 1989] use Cartesian stiffness matrix or its mathematical properties (such as determinant, trace, norm, and etc) as evaluation indexes. For massless cable assumption, the static stiffness of CDPRs only depends on the axial stiffness of cables. In linear-elastic range of the cable, the axial stiffness is independent of cable forces. Thus the Cartesian stiffness matrix of the robot is independent of the external wrench applied to the end-effector. It is easy to compute the Cartesian stiffness matrix through the Jacobian matrix of CDPRs. However, considering the effect of cable weight, the cable profile between two attachment points is not a straight line but a sagging curve. So the direction of cable force is not along the chord of the curve but tangential to the sagging curve. In this case, Jacobian matrix cannot be used to calculate the Cartesian stiffness matrix, and partial differential equations should be employed instead [Arsenault 2013]. This increases calculation complexity. Furthermore, taking the cable sag into consideration, the stiffness of cables is relevant to cable forces and thus depends on the external payload.

\section{Dynamic stiffness}

Although there are lots of researches on the vibration analysis and control for rigid-link parallel robots [Kozak+ 2004; Piras+ 2005; Yun+ 2010; Wang+ 2006; Gexue+ 2004; Algermissen+ 2005; Hesselbach+ 2004; Kang+ 2005; Zhang+ 2008; Mitsuta+ 1994], only few studies can be found on the vibration analysis of CDPRs [Diao+ 2009; Ma+ 2005; Tang+ 2013; Weber+ 2014; Kozak+ 2006; Du+ 2012; Du+2 2013; Kozak+ 2006]. Because CDPRs are quite new in the big family of robotics, most researches remain on the design, simulation and prototype manufacturing. Recently more and more CDPRs are designed and built aiming to be used in real applications. Some of them require high performances, especially the dynamic performances. For examples: the ultrahigh speed FALCON robot [Kawamura+ 1995; Kawamura+ 2000], the wind-induced vibration problem of the large radio telescope $[\mathrm{Zi}+2008]$ and the wind tunnels (fig. 1.9c) [Bruckmann+ 2010]. Vibration can be induced by initial position and velocity of the end-effector, wind disturbance, and/or friction of the cables around fixed pulleys [Du+ 2012]. Vibration can affect the positioning accuracy of the end-effector, and bring fluctuation on the trajectory. These applications lead to researches on the dynamic stiffness and vibration problem of CDPRs.

The vibration of CDPRs can be affected by the compliance of the driving cables, the actuators and the end-effector. Compared with cables, the compliance of the actuators and the end-effector is much lower and therefore can be neglected. Thus the compliance of cables is the primary reason for the vibration of CDPRs. Cable vibration, the rigid-body modes of the end-effector suspended on the stiffness of the cables and their coupling should be 
considered in the dynamic analysis of CDPRs.

Cables have been modeled as taught strings, and end-effector vibration caused by axial and transversal cable flexibility has been analyzed by simulations in [Diao+ 2009; Ma+ 2005]. Cables are modeled as linear massless axial springs, and vibration characteristics of a CDPR for processing applications are presented in [Tang+ 2013]. Vibrations are analyzed based on the linear spring cable model, and a new approach using reaction wheels to compensate the rotational oscillations of the end-effector is proposed in [Weber+ 2014]. Finite element method has been used in the modeling of cable dynamics, and the end-effector vibration together with the system natural frequencies have been studied by simulations in [Du+ 2013; Du+ 2012]. Robot stiffness matrix has been deduced and system natural frequencies have been calculated in [Kozak+ 2006].

Most of these researches [Diao+ 2009; Ma+ 2005; Tang+ 2013; Weber+ 2014] only consider cable elasticity, while neglecting the effect of cable mass on the cable dynamics. Although the effect of cable weight on the static cable profile is considered for the static analysis in [Kozak+ 2006], the effect of cable mass on the cable dynamics is totally ignored in the computation of robot natural frequencies. The effect of cable mass on the cable dynamics is taken into account in the finite element cable model in [Du+ 2012; Du+ 2013]. However, this cable model is based on the Finite Element Method (FEM), and it uses distributed mass points and ideal lines between them to simulate continuous cable. Thus it leads to a very complex system with many partial differential equations. Moreover, as it is known, the accuracy of finite element method depends on the number of elements. To ensure a good accuracy will result in further computational complexity.

To sum up, some researches consider the effect of cable mass on the cable dynamics, while most researches neglect this effect. Thus, whether the effect of cable mass on the cable dynamics is significant for the robot dynamics or not still remains unknown in the existing literatures. Moreover, how to set up an complete dynamic cable model that considers the cable dynamics, the end-effector vibrations and their coupling is still a challenge. In addition, experimental validation should be also considered.

\subsection{Objectives of this thesis}

The main objective of this thesis is to analyze the static and dynamic stiffness of CDPRs with considering the effect of cable sagging and cable dynamic, aiming to improve their static positioning accuracy and trajectory tracking for high dynamic applications. To achieve this objective, the following sub-objectives are made. 


\section{Static stiffness and positioning accuracy analysis of CDPRs}

The primary task of static stiffness analysis is the cable modeling. This thesis reviews the sagging cable model and formulates the static stiffness matrix of a single cable, which contains not only the axial compliance of cables but also the sag-introduced compliance. In addition, experimental validation of the sagging cable model is achieved on a 6-DOF CDPR prototype. The effect of cable weight of the static cable profile is verified.

Another objective of static analysis is the evaluation of the static stiffness performance. In this thesis, the static pose error of the end-effector is defined and calculated based on the kinematic model of CDPRs, and the variation of the end-effector pose error with the external load is used to evaluate the static stiffness of CDPRs. Experiments are made on a CDPR prototype. The variation of the pose error along the vertical direction is studied by loading the end-effector with different payloads. Through comparisons of the simulation results and experimental data, the effect of cable sag on the static positioning accuracy of CDPRs is verified.

\section{Dynamic stiffness and vibration analysis of CDPRs}

In order to consider the effect of cable mass on the cable dynamics, the Dynamic Stiffness Matrix (DSM) method is used to formulate the dynamic stiffness matrix of an inclined sagging cable. DSM is used to solve the vibration problems of structures. It is often regarded as an exact method, because DSM is based on the exact shape functions obtained from the exact solution of the element differential equations [Ansell 2005].

It is well recognized that the Finite Element Method (FEM) is a very powerful numerical method in the structural dynamic analysis [Hughes 2012]. The FEM models a structure as an assemblage of small elements. Each element has a simple geometry and thus is much easier to be studied than the actual structure. The FEM is often regarded as an approximate method because it represents the continuum with an assemblage of discrete elements where discretization errors are unavoidable. Moreover, the properties of an individual element are derived from the assumed shape functions [Zhou 1996]. Therefore, the accuracy of the FEM depends on the number of discrete elements and the properties of the shape functions. To improve the precision, a usual way is to add the number of the discrete elements, which can directly increase the computation complexity.

However, the DSM method can be regarded as an exact method which provides better accuracy compared with the FEM [Banerjee 1997; Koloušek+ 1973; Åkesson 1976; Leung 1978]. It employs the frequency-dependent shape functions that are exact solutions of the 
governing differential equations, and thus provides exact natural modes for a vibrating structure. The DSM method can eliminates the spatial discretization error and is capable to predict infinite number of natural modes by means of a small number of DOFs, which can significantly decrease the computation complexity. In some situations, only one element is required to calculate any desired number of frequencies. Therefore, the number of nodes or DOFs in the DSM method is much less than that in the conventional FEM [Zhou 1996]. The DSM method is specially useful for the analysis of curved structures where the entire length of the structure can be modeled as just one element while without losing accuracy, such as the inclined cable structure. Because the shape functions used in the DSM method are dependent of frequency, the resulted dynamic stiffness matrix of an inclined sagging cable is inherently frequency-dependent.

In order to study the effect of cable dynamics on the dynamic behaviors of CDPRs, a new dynamic model of CDPRs is proposed with considering the coupling of cable dynamics and end-effector vibrations. The dynamic stiffness matrix of CDPRs is computed according to the dynamic cable model and geometric relationship. This dynamic matrix is an assemblage of the dynamic stiffness matrix of all the driving cables. It considers the cable elasticity and the effect of cable mass on the cable dynamics. With this dynamic stiffness matrix, the oscillating equations of the end-effector around a static equilibrium are formulated through the Lagrange's equations. Dynamic response functions of the end-effector under a harmonic excitation are used to identify the natural frequencies of CDPRs, and to study the coupling of the cable dynamics and the end-effector vibrations.

A CDPR prototype with 6-DOF driven by 8 cables, the CoGiRo [Lamaury 2013], is used in the dynamic experimental validation. Modal experiments, free vibration experiments and trajectory experiments are carried out to validate the introduced dynamic cable model and the proposed dynamic stiffness model of CDPRs, also analyze the coupling between the cable dynamics and the end-effector vibration.

\section{Application of the proposed method on the force distribution of CDPRs}

Besides stiffness analysis, the proposed models are applied on the force distribution of redundant actuated CDPRs. Previous studies on this issue [Gosselin+ 2011; Mikelsons+ 2008; Pott+ 2009; Khosravi+ 2013; Oh+ 2005; Bruckmann+ 2006; Kawamura+ 1995; Fang+ 2004; Hassan+2008; Lim+ 2011] usually neglect the effect of cable weight on the cable profile and/or the cable elasticity, where cables are assumed as massless straight lines. Besides, there is no literature on the determination fo the lower-boundary of cable forces.

In this thesis, the proposed models of cables and CDPRs consider the effect of cable 
weight on the static cable profile, the effect of cable mass on the cable dynamics and the cable elasticity. The static and dynamic behaviors of CDPRs have been analyzed in the first several sections of this thesis. Therefore, it is not difficult to apply the proposed models and methods in the force distribution of redundant actuated CDPRs to improve the static and dynamic performances of CDPRs.

The force distribution method considering the effect of cable weight on the static cable profile is presented. With cable sag, the kinematics and force distribution of CDPRs are coupled. The proposed method solves the coupling problem by using optimization algorithms. Methods on the determination of the lower-boundary of cable forces are presented, and a new pose-dependent force boundary method is proposed based on the dynamic cable model presented in the beginning of this thesis. The lower-boundary of each driving cable is calculated for every pose of the end-effector along a trajectory. Compared with the traditional fixed lower-boundary method, the proposed pose-dependent lower-boundary method can give out much more suitable force boundaries for every cable. Thus it can guarantee the cable performance according to the design requirement while not stretch the cable too much. 


\section{Chapter 2}

\section{Static and Dynamic Modeling of Cables}

\section{Contents}

2.1 Static cable modeling $\ldots \ldots \ldots \ldots$

2.2 Dynamic cable modeling $\ldots \ldots \ldots \ldots \ldots$

2.3 A numerical example . . . . . . . . . . . . . . 29

2.4 Summary of the chapter . . . . . . . . . . . . . 34

Cable behaviors will affect the performances of CDPRs, such as the positioning accuracy and vibration problem. In order to get better understanding of CDPRs, cables should be studied first. This chapter focuses on the cable modeling.

In many previous researches [Kawamura+ 1995; Gouttefarde+ 2006; Berti+ 2013], cables are modeled as ideal lines. The static profile of an ideal cable between two points is a straight line without elongation or contraction. The cable length is independent with the cable tension. This model neglects the cable elasticity and the effect of cable weight on the static cable profile. The ideal cable model is the simplest cable model which is often used in the theoretical analysis of CDPRs.

Another common model is the spring cable model [Dagalakis+ 1989; Verhoeven+ 1998; Vafaei+ 2011; Khosravi+ 2013]. This model considers the cable elasticity while neglecting the effect of cable weight on the static cable profile. The static cable profile between two points is also a straight line but with elongation. The strained cable length depends on the cable tension and the cable elasticity. Mathematically their relationship can be described by the Hooke's law: $F=k \Delta X$, where $F$ represents the cable force, $\Delta X$ represents the elongation of the cable length, and $k$ is the stiffness coefficient. $k$ is closely relevant to the material characteristics, such as the Young 's modulus. If $k$ is assumed to be constant, the model is a 
linear spring model. If $k$ is not constant, the model is a non-linear spring model [Riehl 2011]. According to [Riehl 2011], the non-linearity of cables comes from the material, the cable structure and the range of the external load. The basic assumption of the spring model is that the effect of cable weight on the static cable profile is negligible. Therefore, spring cable model is valid and efficient for low-density and thin cables. If the cable weight is significant compared with the external load, the effect of cable weight on the static cable profile cannot be neglected. In this situation, spring model may lead to unacceptable errors.

A third model is the sagging cable model which derives from the civil engineering [Irvine 1992]. This model considers not only the cable elasticity but also the effect of cable weight on the static cable profile. It describes the cable profile with a set of non-linear equations. The sagging cable model has been used in several previous researches [Kozak+ 2006; Riehl+ 2009; Gouttefarde+ 2012; Sandretto+ 2013b; Arsenault 2013]. Although the sagging cable model is more accurate than the ideal and spring cable models, it is also more complex in computation, which is a big challenge for real-time applications.

The three cable models above are used for the static analyses of CDPRs. For the dynamic and vibration analyses of CDPRs, cables are often modeled as massless springs with a linear axial elasticity [Tang+ 2013; Ma+ 2005; Diao+ 2009; Weber+ 2014]. This cable model neglects the vibration of cables and only considers the rigid-body modes of the end-effector suspended on the stiffness of the cables. Another dynamic cable model is the DSM cable model [Starossek 1991b; Ansell 2005], which uses the dynamic stiffness matrix to describe cable dynamics. The DSM cable model is based on the DSM method and it considers the cable elasticity and the effect of cable mass on the cable dynamics. The DSM method can be regarded as an exact method [Banerjee 1997]. It employs the frequency-dependent shape functions that are exact solutions of the governing differential equations, and thus provides exact natural modes for a vibrating structure [Zhou 1996]. The DSM method is specially useful for the analysis of curved structures where the entire length of the structure can be modeled as just one element while without losing accuracy, such as the inclined cable structure.

In this chapter, the effect of cable weight on the static cable profile and the effect of cable mass on the cable dynamics are considered in the static and dynamic cable modeling, respectively, aiming at the precise analysis of cable behavior. For the static cable modeling, the static sagging cable model is firstly reviewed. Parameters associated with the static cable characteristics are also calculated. Based on this sagging model, static stiffness matrix of an inclined sagging cable is then deduced. For the dynamic cable modeling, the dynamic stiffness matrix of a horizontal sagging cable is derived, and then it is extended to an inclined 
sagging cable. At the end of this chapter, an example is given to illustrate how to use the proposed cable models in the analysis of static and dynamic cable performances.

\subsection{Static cable modeling}

\subsubsection{Profile of a static sagging cable}

The static sagging cable model, also known as elastic catenary model, considers the cable elasticity and the effect of cable weight on the static cable profile. It describes the static profile of a cable by a set of non-linear equations. This model has been studied and used in civil engineering since 1930s [Irvine 1992]. However, it is quite new in the analysis of CDPRs [Riehl+ 2009; Kozak+ 2006]. In addition, this cable model is the theoretical basis of this thesis. It is necessary to briefly introduce this model with variables familiar to robotics. In this thesis, the non-linearity due to axial cable elasticity is neglected in the mathematical modeling and the experimental validation. According to the results from a material testing machine, the anti-rust steel cables used in this thesis show negligible non-linearity along the axial direction in the working load range.

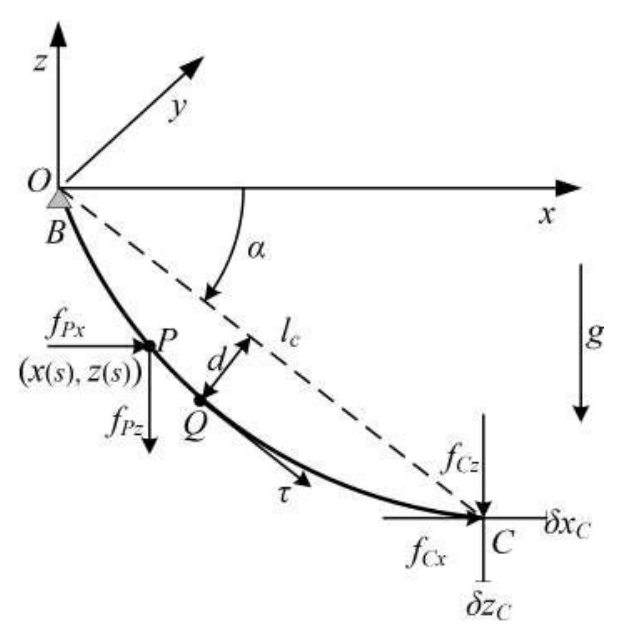

Fig. 2.1 Diagram of a sagging cable

The diagram of an inclined cable is presented in Fig. 2.1. One of the cable-ends is fixed, and an external force is applied to the other end. With the effect of both external force and gravity, the shape of the cable between points $C$ and $B$ is not a straight line, but a sagging curve in the $x O z$ plane.

Assuming that $P$ is an arbitrary point on the cable, the constraints for a differential cable element around the point $P$ are: 
1. The geometric constraint:

$$
\left(\frac{d x}{d p}\right)^{2}+\left(\frac{d z}{d p}\right)^{2}=1
$$

2. The constraint of static equilibrium:

$$
\begin{aligned}
& f_{P} \frac{d x}{d p}=f_{P x}=f_{C x}, \\
& f_{P} \frac{d z}{d p}=f_{P z}=f_{C z}-\rho g\left(l_{u s}-s\right) .
\end{aligned}
$$

3. The constraint according to the Hooke's law:

$$
f_{P}=E A\left(\frac{d p}{d s}-1\right)
$$

where:

- $(x, z)$ is the Cartesian coordinate of point $P$ in frame $\Re_{O}(O, x, y, z)$;

- $l_{u s}$ and $l_{s}$ are the unstrained and strained cable length between points $C$ and $B$ respectively;

- $s$ and $p$ are the Lagrangian coordinate in the unstrained and strained cable profile respectively, where $0 \leq s \leq l_{u s}$ and $0 \leq p \leq l_{s}$;

- $f_{C}$ is the cable force on point $C ; f_{C x}$ and $f_{C z}$ are the components of $f_{C}$ along $x$ and $z$ axis respectively;

- $f_{P}$ is the cable force on point $P ; f_{P x}$ and $f_{P z}$ are the components of $f_{P}$ along $x$ and $z$ axis respectively;

- $\rho$ is the cable mass per unit length;

- $g$ is the gravitational acceleration;

- $A$ is unstrained cross-sectional area;

- $E$ is the Young's modulus. 
According to eqs. (2.1) to (2.3), the force on point $P$ can be written as:

$$
f_{P}=\sqrt{f_{P x}^{2}+f_{P z}^{2}}=\sqrt{f_{C x}^{2}+\left[f_{C z}-\rho g\left(l_{u s}-s\right)\right]^{2}} .
$$

Since both $\frac{d x}{d p}$ and $\frac{d p}{d s}$ can be written as functions of $f_{P}$ by eqs. (2.2) and (2.4), $\frac{d x}{d s}$ can be written as:

$$
\frac{d x}{d s}=\frac{d x}{d p} \frac{d p}{d s}=\frac{f_{C x}}{f_{P}}\left(\frac{f_{P}}{E A}+1\right)
$$

Substituting eq. (2.5) into eq. (2.6) yields:

$$
\frac{d x}{d s}=\frac{f_{C x}}{E A}+\frac{f_{C x}}{\sqrt{f_{C x}^{2}+\left[f_{C z}-\rho g\left(l_{u s}-s\right)\right]^{2}}} .
$$

Applying the same procedures for $z$-axis, we can get:

$$
\begin{aligned}
\frac{d z}{d s} & =\frac{d z}{d p} \frac{d p}{d s}=\frac{f_{P z}}{f_{P}}\left(\frac{f_{P}}{E A}+1\right) \\
& =\frac{f_{C z}-\rho g\left(l_{u s}-s\right)}{E A}+\frac{f_{C z}-\rho g\left(l_{u s}-s\right)}{\sqrt{f_{C x}^{2}+\left[f_{C z}-\rho g\left(l_{u s}-s\right)\right]^{2}}} .
\end{aligned}
$$

After integration with the boundary condition $x(0)=0$ and $z(0)=0$, the static cable profile can be described as:

$$
\begin{aligned}
x(s) & =\frac{f_{C x} s}{E A}+\frac{\left|f_{C x}\right|}{\rho g}\left\{\sinh ^{-1}\left[\frac{f_{C z}-\rho g\left(l_{u s}-s\right)}{f_{C x}}\right]-\sinh ^{-1}\left(\frac{f_{C z}-\rho g l_{u s}}{f_{C x}}\right)\right\} \\
z(s) & =\frac{f_{C z} s}{E A}+\frac{\rho g}{E A}\left(\frac{s^{2}}{2}-l_{u s} s\right) \\
& +\frac{1}{\rho g}\left\{\sqrt{f_{C x}^{2}+\left[f_{C z}-\rho g\left(l_{u s}-s\right)\right]^{2}}-\sqrt{f_{C x}^{2}+\left(f_{C z}-\rho g l_{u s}\right)^{2}}\right\} .
\end{aligned}
$$

\subsubsection{Calculation of the static cable parameters}

According to the static sagging cable model, some cable parameters can be calculated (see fig. 2.1), such as $x_{C}$ and $z_{C}$ (the coordinates of the end point), $l_{c}$ (the chord length), $\alpha$ (the inclined angle), $\tau$ (the static cable tension at the section where the cable is parallel to the chord), and $d$ (the sag perpendicular to the chord at point $Q$ ). These parameters describe the static cable characteristics, and they are useful for the static analysis of sagging cable. Moreover, these parameters will be later proved important in the analysis of cable dynamics. The calculations are presented as following: 
1. The coordinates of the end point of the cable $C$ can be obtained by substituting $s=l_{u s}$ to eqs. (2.9) and (2.10):

$$
\begin{aligned}
& x_{C}=x\left(l_{u s}\right)=\frac{f_{C x} l_{u s}}{E A}+\frac{\left|f_{C x}\right|}{\rho g}\left\{\sinh ^{-1}\left(\frac{f_{C z}}{f_{C x}}\right)-\sinh ^{-1}\left(\frac{f_{C z}-\rho g l_{u s}}{f_{C x}}\right)\right\}, \\
& z_{C}=z\left(l_{u s}\right)=\frac{f_{C z} l_{u s}}{E A}-\frac{\rho g l_{u s}^{2}}{2 E A}+\frac{1}{\rho g}\left\{\sqrt{f_{C x}^{2}+f_{C z}^{2}}-\sqrt{f_{C x}^{2}+\left(f_{C z}-\rho g l_{u s}\right)^{2}}\right\} .
\end{aligned}
$$

2. As shown in fig. 2.1, the coordinate of the fixed point $B$ is $(0,0)$. Then the chord length and the inclined angle of the sagging cable can be obtained by:

$$
\begin{aligned}
& l_{c}=\sqrt{\left(z_{C}-z_{B}\right)^{2}+\left(x_{C}-x_{B}\right)^{2}}=\sqrt{z_{C}^{2}+x_{C}^{2}}, \\
& \alpha=\arctan \left(\left|\frac{z_{C}-z_{B}}{x_{C}-x_{B}}\right|\right)=\arctan \left(\left|\frac{z_{C}}{x_{C}}\right|\right) .
\end{aligned}
$$

3. The strained cable length $l_{s}$ can be obtained by the integration of eqs. (2.9) and (2.10):

$$
l_{s}=\int_{0}^{l_{u s}} \sqrt{\left(\frac{d x}{d s}\right)^{2}+\left(\frac{d z}{d s}\right)^{2}} d s
$$

and the cable elongation $\Delta l$ can be written as:

$$
\Delta l=l_{s}-l_{u s}
$$

4. According to the geometric relationship $\frac{x_{C}}{l_{c}}=\frac{\tau_{x}}{\tau}$, and the static equilibrium of the cable $\tau_{x}=f_{C x}$, the static cable tension at the section where the cable is parallel to the chord can be expressed as:

$$
\tau=\frac{f_{C x} l_{c}}{x_{C}}
$$

5. In order to calculate the cable sag $d$, the Lagrange coordinate of point $Q$ is firstly computed, where $Q$ is a point on the cable where the cable tension is parallel to the chord (see fig. 2.1). According to the geometric relationship:

$$
\frac{\tau_{z}}{\tau_{x}}=\frac{z_{C}-z_{B}}{x_{C}-x_{B}}=\frac{z_{C}}{x_{C}}
$$


According to the static equilibrium of the cable:

$$
\begin{aligned}
\tau_{z} & =f_{C z}+\rho g\left(s_{Q}-l_{u s}\right), \\
\tau_{x} & =f_{C x} .
\end{aligned}
$$

Substituting eqs. (2.19) and (2.20) to eq. (2.18), the Lagrange coordinate $s_{Q}$ can be obtained:

$$
s_{Q}=\frac{f_{C x} z_{C}-f_{C z} x_{C}}{\rho g x_{C}}+l_{u s} .
$$

The Cartesian coordinates of point $Q$ can be obtained by substituting eq. (2.21) to eqs. (2.9) and (2.10). The equation of the chord can be express as: $z_{C} x-x_{C} z=0$. The sag perpendicular to the chord is equal to the distance from the point $Q$ to the chord, therefore:

$$
d=\frac{\left|z_{C} x_{Q}-x_{C} z_{Q}\right|}{\sqrt{z_{C}^{2}+x_{C}^{2}}}=\frac{\left|z_{C} x_{Q}-x_{C} z_{Q}\right|}{l_{c}} .
$$

\subsubsection{Static stiffness matrix of a sagging cable}

Stiffness describes the capacity of a structure to resist the deformations induced by the applied loads. For the spring cable model without considering the effect of cable weight on the static cable profile, the axial cable stiffness is the only factor that should be taken into account in the formulation of the stiffness matrix. However, for sagging cable model, a second factor, sag-introduced flexibility, must also be considered. The axial cable stiffness is caused by the axial elasticity of cable, while the sag-introduced flexibility is due to the gravitational potential energy stored in the cable. The static stiffness of a sagging cable can be defined as the ratio of the force required to create a specified deflection or movement of a part. Based on the static sagging cable model established in section 2.1.1, the static stiffness matrix is formulated as:

$$
\left[\begin{array}{c}
f_{C x} \\
f_{C z}
\end{array}\right]=\mathbf{K}_{\text {static }}^{2 \mathrm{D}}\left[\begin{array}{c}
\delta x_{C} \\
\delta z_{C}
\end{array}\right]
$$

where $\left[f_{C x}, f_{C z}\right]$ represent the forces applied at the end point of the cable, and $\left[\delta x_{C}, \delta z_{C}\right]$ represent the differential displacements at the same point (fig. 2.1), and $\mathbf{K}_{\text {static }}^{2 \mathrm{D}}$ is the 2 by 2 stiffness matrix. It should be noted that $\left[\delta x_{C}, \delta z_{C}\right]$ are defined as differentials that represent 
small changes of position from the static equilibrium.

In order to calculate the stiffness matrix $\mathbf{K}_{\text {static }}^{2 \mathrm{D}}$, the compliance matrix $\mathbf{C}_{\text {static }}^{2 \mathrm{D}}$ is firstly obtained by computing the differentiation of eqs. (2.11) and (2.12) with respect to $f_{C x}$ and $f_{C z}$ :

$$
\begin{aligned}
& C_{x x}^{2 \mathrm{D}}=\frac{\partial x_{C}}{\partial f_{C x}}=\frac{l_{u s}}{E A}+\frac{1}{p g}\left\{\operatorname{sign}\left(f_{C x}\right)\left[\sinh ^{-1}\left(\frac{f_{C z}}{f_{C x}}\right)-\sinh ^{-1}\left(\frac{f_{C z}-p g l_{u s}}{f_{C x}}\right)\right]\right. \\
& \left.-\frac{f_{C z}}{\sqrt{f_{C x}^{2}+f_{C z}^{2}}}+\frac{f_{C z}-p g l_{u s}}{\sqrt{f_{C x}^{2}+\left(f_{C z}-p g l_{u s}\right)^{2}}}\right\} \\
& C_{x z}^{2 \mathrm{D}}=C_{z x}^{2 \mathrm{D}}=\frac{\partial x_{C}}{\partial f_{C z}}=\frac{\partial z_{C}}{\partial f_{C x}}=\frac{1}{p g}\left[\frac{f_{C x}}{\sqrt{f_{C x}^{2}+f_{C z}^{2}}}-\frac{f_{C x}}{\sqrt{f_{C x}^{2}+\left(f_{C z}-p g l_{u s}\right)^{2}}}\right], \\
& C_{z z}^{2 \mathrm{D}}=\frac{\partial z_{C}}{\partial f_{C z}}=\frac{l_{u s}}{E A}+\frac{1}{p g}\left[\frac{f_{C z}}{\sqrt{f_{C x}^{2}+f_{C z}^{2}}}-\frac{f_{C z}-p g l_{u s}}{\sqrt{f_{C x}^{2}+\left(f_{C z}-p g l_{u s}\right)^{2}}}\right],
\end{aligned}
$$

where $C_{i j}^{2 \mathrm{D}}$ is the element of matrix $\mathbf{C}_{\text {static }}^{2 \mathrm{D}}$. As explained in the beginning of this section, two sources should be considered to formulate of the stiffness matrix of a sagging cable. One is the axial cable elasticity, the other is the sag-introduced flexibility. This point can be further proved by separating the compliance matrix $\mathbf{C}_{\text {static }}^{2 \mathrm{D}}$ in eqs. (2.25) to (2.27) into two parts:

$$
\begin{aligned}
\mathbf{C}_{\text {static }}^{2 \mathrm{D}} & =\mathbf{C}_{\mathrm{axial}}^{2 \mathrm{D}}+\mathbf{C}_{\mathrm{sag}}^{2 \mathrm{D}} \\
& =\left[\begin{array}{cc}
\frac{l_{u s}}{E A} & 0 \\
0 & \frac{l_{u s}}{E A}
\end{array}\right]+\left[\begin{array}{cc}
C_{x x}^{2 \mathrm{D}}-\frac{l_{u s}}{E A} & C_{x z}^{2 \mathrm{D}} \\
C_{z x}^{2 \mathrm{D}} & C_{z z}^{2 \mathrm{D}}-\frac{l_{u s}}{E A}
\end{array}\right] .
\end{aligned}
$$

The static stiffness matrix of the cable $\mathbf{K}_{\text {static }}^{2 \mathrm{D}}$ can then be obtained by:

$$
\mathbf{K}_{\text {static }}^{2 \mathrm{D}}=\operatorname{inv}\left(\mathbf{C}_{\text {static }}^{2 \mathrm{D}}\right)=\frac{1}{C_{x x}^{2 \mathrm{D}} C_{z z}^{2 \mathrm{D}}-C_{x z}^{2 \mathrm{D}} C_{z x}^{2 \mathrm{D}}}\left[\begin{array}{cc}
C_{x x}^{2 \mathrm{D}} & -C_{x z}^{2 \mathrm{D}} \\
-C_{z x}^{2 \mathrm{D}} & C_{z z}^{2 \mathrm{D}}
\end{array}\right] .
$$

In eq. (2.28), $\mathbf{C}_{\mathrm{axial}}^{2 \mathrm{D}}$ is the compliance matrix caused by the axial cable elasticity, which is associated with the physical cable parameters. $\mathbf{C}_{\text {sag }}^{2 \mathrm{D}}$ is the compliance matrix caused by the sag-introduced flexibility, which is not only related to the cable parameters but also related to the external forces applied to the cable end. This issue will be studied in details in the following chapters. 


\subsection{Dynamic cable modeling}

The Dynamic Stiffness Matrix method is used to solve the vibration problems of structures. It can provide a good accuracy and it is often regarded as an exact method, because DSM is based on the exact shape functions obtained from the exact solution of the element differential equations [Ansell 2005]. In this section, the dynamic stiffness matrix of a horizontal sagging cable is firstly presented. Then this method is extended to an inclined sagging cable.

\subsubsection{Notifications and assumptions}

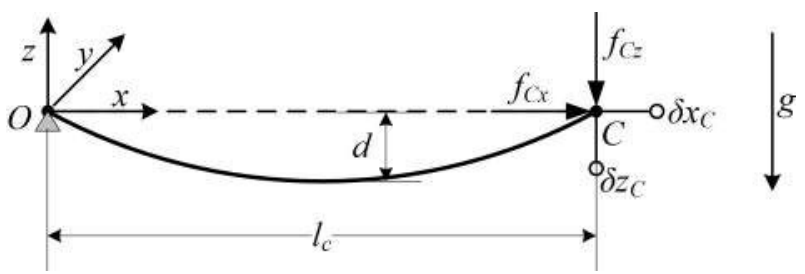

Fig. 2.2 Diagram of a horizontal sagging cable in the cable plan [Starossek 1991b]

Figure 2.2 shows the diagram of a horizontal sagging cable, where $d$ is the sag perpendicular to the chord; $l_{c}$ is the chord length; $f_{C x}$ and $f_{C z}$ are the dynamic forces; $\delta x_{C}$ and $\delta z_{C}$ are the dynamic displacements around point $C$. The dynamic cable model is based on the linear theory of free vibrations. According to [Kim+ 2001; Ansell 2005; Starossek 1991b], the following assumptions are made:

- The cable is assumed to be continuum and uniform [Starossek 1991b];

- Only small displacements are admitted to meet the requirement of linear theory [Bellet 1988];

- Only small cable sag is allowed, where $\frac{d}{l_{c}}$ (the sag to span ratio) is no more than $1 / 20$ [Starossek 1991b];

- Viscous damping is taken into consideration.

\subsubsection{Dynamic stiffness matrix of a horizontal sagging cable}

In linear theory the in-plane motion of a cable is uncoupled with the out-of-plane motion [Ansell 2005; Kim+ 2001]. For the sake of convenience, the dynamic stiffness matrix is 
firstly deduced in the cable plane, then it will be extended to 3 dimensions without major difficulty.

As shown in fig. 2.2, the dynamic stiffness matrix of a horizontal sagging cable expressed in the cable plan $\mathbf{K}_{\text {dyn-h }}^{2 \mathrm{D}}(\omega)$ can be defined as:

$$
\left[\begin{array}{c}
f_{C x} \\
f_{C z}
\end{array}\right]=\mathbf{K}_{\mathrm{dyn}-\mathrm{h}}^{2 \mathrm{D}}(\omega)\left[\begin{array}{c}
\delta x_{C} \\
\delta z_{C}
\end{array}\right]
$$

where $\left[\begin{array}{c}f_{C x} \\ f_{C z}\end{array}\right]$ and $\left[\begin{array}{c}\delta x_{C} \\ \delta z_{C}\end{array}\right]$ are the vectors of the dynamic forces and displacements shown in fig. 2.2. According to [Starossek 1991a], the dynamic stiffness matrix $\mathbf{K}_{\text {dyn-h }}^{2 \mathrm{D}}(\boldsymbol{\omega})$ can be formulated as:

$$
\mathbf{K}_{\mathrm{dyn}-\mathrm{h}}^{2 \mathrm{D}}(\omega)=\left[\begin{array}{cc}
K_{x x}(\omega) & K_{x z}(\omega) \\
K_{z x}(\omega) & K_{z z}(\omega)
\end{array}\right]
$$

where:

$$
\begin{aligned}
& K_{x x}(\omega)=\frac{E A}{L_{e}} \frac{1}{1+\frac{\lambda^{2}}{\Omega_{c}^{2}}(\kappa-1)}, \\
& K_{x z}(\omega)=K_{z x}(\omega)=\frac{E A}{L_{e}} \frac{\frac{1}{2} \varepsilon(\kappa-1)}{1+\frac{\lambda^{2}}{\Omega_{c}^{2}}(\kappa-1)}, \\
& K_{z z}(\omega)=\frac{E A}{L_{e}} \frac{\varepsilon^{2}}{\lambda^{2}} \frac{1}{\kappa}-\frac{E A}{L_{e}} \frac{\frac{1}{4} \frac{\varepsilon^{2}}{\lambda^{2}} \Omega_{c}^{2}\left[\kappa+\frac{\lambda^{2}}{\Omega_{c}^{2}}(\kappa-1)\right]}{1+\frac{\lambda^{2}}{\Omega_{c}^{2}}(\kappa-1)} .
\end{aligned}
$$

The relative parameters in eqs. (2.32) to (2.34) are:

- $\lambda^{2}=\left(\frac{\rho g l_{c}}{H}\right)^{2} \frac{E A l_{c}}{H L_{e}}$ is the fundamental cable parameter which represents the elastic stiffness relative to the catenary stiffness;

- $\varepsilon=\frac{\rho g l_{c}}{H}=\frac{8 d}{l_{c}}$ is the ratio between horizontal cable weight and cable tension;

- $L_{e}=\int_{0}^{l_{c}}\left(\frac{d s}{d x}\right)^{3} d x \simeq l_{c}\left[1+8\left(\frac{d}{l_{c}}\right)^{2}\right]$ is the cable length parameter;

- $\Omega=\omega l_{c} \sqrt{\frac{\rho}{H}}$ is the dimensionless frequency parameter;

- $\Omega_{c}=\omega_{c} l_{c} \sqrt{\frac{\rho}{H}}$ is the dimensionless frequency-damping parameter;

- $\kappa=\frac{\tan \left(\frac{\Omega_{c}}{2}\right)}{\left(\frac{\Omega_{c}}{2}\right)}$ is an auxiliary term. 
As we explained before, the in-plane motion of a sagging cable is uncoupled with the out-of-plane motion. Similar to the deduction of the planar dynamic stiffness matrix, with consideration of the out-of-plane motion (cable motion along $y$-axis that is perpendicular to the cable plane), the spatial dynamic stiffness matrix of a horizontal sagging cable in 3 dimensions can be expressed as:

$$
\mathbf{K}_{\text {dyn-h }}^{3 \mathrm{D}}(\omega)=\left[\begin{array}{ccc}
K_{x x}(\omega) & 0 & K_{x z}(\omega) \\
0 & K_{y y}(\omega) & 0 \\
K_{z x}(\omega) & 0 & K_{z z}(\omega)
\end{array}\right]
$$

Due to the uncoupling between the in-plane motion and the out-of-plane motion, the interaction coefficients in eq. (2.35) are zeros. According to [Ansell 2005], the stiffness matrix coefficient for the out-of-plane motion is $K_{y y}(\omega)=\frac{\tau\left(4-\kappa^{2} \Omega_{c}^{2}\right)}{4 \kappa l_{c}}$. The other coefficients are the same with those in eq. (2.31).

\subsubsection{Dynamic stiffness matrix of an inclined sagging cable}

The theory developed for the dynamic analysis of a horizontal sagging cable in section 2.2.2 can be extended to study the dynamics of an inclined sagging cable [Irvine 1978; Starossek 1991b].

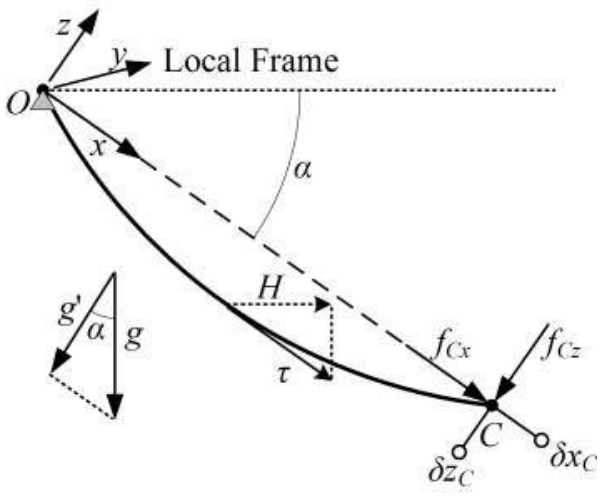

(a) In the local cable frame

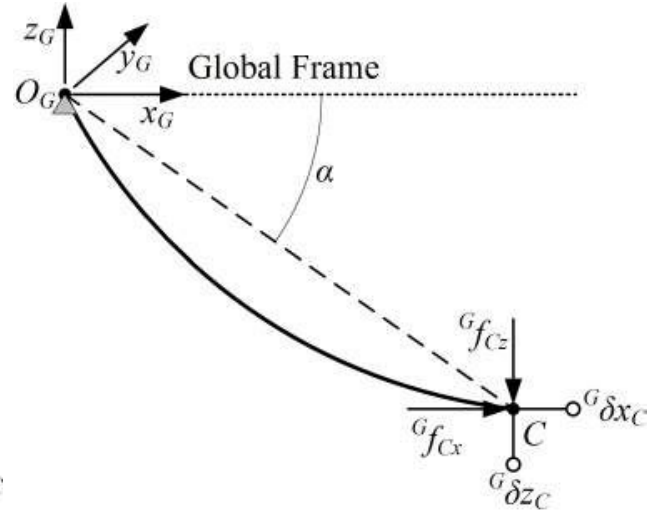

(b) In the global frame

Fig. 2.3 Forces and displacements of an inclined sagging cable

Figure 2.3a represents the forces and displacements of an inclined sagging cable in the local cable frame, where axis- $x$ is parallel to the chord. It can be obtained by rotating the horizontal sagging cable (shown in fig. 2.2) around axis-y, where the rotation angle is $\alpha$. The extension to an inclined sagging cable is made by the following substitutions. Firstly, the 
gravity $g$ is replaced by the gravitational component $g^{\prime}$, where $g^{\prime}$ is perpendicular to the cable chord and $g^{\prime}=g \cos \alpha$. Secondly, the horizontal static cable tension $H$ is replaced by $\tau$. $\tau$ represents the static cable tension at the section where the cable is parallel to the chord, and $\tau=\frac{H}{\cos \alpha}$. Parameters related to $g$ and $H$ become:

$$
\begin{aligned}
\lambda^{2} & =\left(\frac{\rho g l_{c}}{\tau}\right)^{2} \frac{E A l_{c}}{\tau L_{e}} \cos ^{2} \alpha, \\
\varepsilon & =\frac{\rho g l_{c}}{\tau} \cos \alpha=\frac{8 d}{l_{c}} \\
\Omega & =\omega l_{c} \sqrt{\frac{\rho}{\tau}} \\
\Omega_{c} & =\omega_{c} l_{c} \sqrt{\frac{\rho}{\tau}}
\end{aligned}
$$

With these new items, the theory for the vibration analysis of a horizontal sagging cable can be used for an inclined sagging cable. It should be noticed that according to [Irvine 1978; Starossek 1991b], this extension is valid when the cable parameter $\lambda^{2}$ and $\varepsilon$ together with the inclined angle $\alpha$ do not exceed certain limits. In particular, $\lambda^{2}$ should maintain a proper distance (about 20\%) from the so-called crossover points $4 n^{2} \pi^{2}(\mathrm{n}=1,2 \ldots)$. To be specific:

$$
\begin{gathered}
\lambda^{2} \leq 24, \\
\text { And } \alpha \leq 60^{\circ}, \varepsilon \leq 0.01\left(\frac{d}{l_{c}} \leq \frac{1}{80}\right) \\
\text { Or } \quad \alpha \leq 30^{\circ}, \varepsilon \leq 0.24\left(\frac{d}{l_{c}} \leq \frac{1}{33}\right)
\end{gathered}
$$

The dynamic stiffness matrix is now prepared to be transformed to the global frame. Figure $2.3 \mathrm{~b}$ represents the forces and displacements of an inclined sagging cable in the global frame $\mathfrak{R}_{G}$, where axis- $z_{G}$ is parallel to the gravity and upward; $O_{G} x_{G} z_{G}$ is in the cable plane. This global frame can be obtained by rotating the local cable frame (in fig. 2.3a) $\alpha$ degree around $y$-axis. The global dynamic stiffness matrix $\mathbf{K}_{\mathrm{dyn}}^{2 \mathrm{D}}(\omega)$ can be defined as:

$$
\left[\begin{array}{l}
{ }^{G} f_{C x} \\
{ }^{G} f_{C z}
\end{array}\right]=\mathbf{K}_{\mathrm{dyn}}^{2 \mathrm{D}}(\omega)\left[\begin{array}{l}
{ }^{G} \delta x_{C} \\
{ }^{G} \delta z_{C}
\end{array}\right],
$$

where $\left[{ }^{G} f_{C x}{ }^{G} f_{C z}\right]^{T}$ and $\left[{ }^{G} \delta x_{C}{ }^{G} \delta z_{C}\right]^{T}$ are the vectors of the dynamic forces and displacements shown in fig. 2.3b. The relationship between $\left[f_{C x} f_{C z}\right]^{T}$ and $\left[{ }^{G} f_{C x}{ }^{G} f_{C z}\right]^{T},\left[\delta x_{C} \delta z_{C}\right]^{T}$ and $\left[{ }^{G} \delta x_{C}{ }^{G} \delta z_{C}\right]^{T}$ can be expressed by the rotation matrix $\mathbf{T}$ that transfers the coordinates 
in the local cable frame to their corresponding coordinates in the global frame:

$$
\begin{aligned}
& {\left[{ }^{G} f_{C x}{ }^{G} f_{C z}\right]^{T}=\mathbf{T}\left[f_{C x} f_{C z}\right]^{T}} \\
& {\left[{ }^{G} \delta x_{C}{ }^{G} \delta z_{C}\right]^{T}=\mathbf{T}\left[\begin{array}{lll}
\delta x_{C} & \delta z_{C}
\end{array}\right]^{T},}
\end{aligned}
$$

where $\mathbf{T}=\left[\begin{array}{cc}\cos \alpha & \sin \alpha \\ -\sin \alpha & \cos \alpha\end{array}\right]$. Therefore, the dynamic stiffness matrix of an inclined sagging cable in the global frame can be obtained by:

$$
\mathbf{K}_{\text {dyn }}^{2 \mathrm{D}}(\omega)=\mathbf{T} \mathbf{K}_{\text {dyn-h }}^{2 \mathrm{D}}(\omega) \mathbf{T}^{-1}
$$

where $\mathbf{K}_{\mathrm{dyn}-\mathrm{h}}^{2 \mathrm{D}}(\omega)$ is defined by eq. (2.31) in section 2.2.2.

In eq. (2.45), $\mathbf{K}_{\text {dyn }}^{2 \mathrm{D}}(\omega)$ is a 2 dimension matrix that only considers the in-plane cable motion. If the out-of-plane cable motion is taken into account, the 3 dimension dynamic stiffness matrix of an inclined sagging cable in the global frame can be obtained through similar coordinate transformation:

$$
\mathbf{K}_{\mathrm{dyn}}^{3 \mathrm{D}}(\omega)=\left[\begin{array}{ccc}
\cos \alpha & 0 & \sin \alpha \\
0 & 1 & 0 \\
-\sin \alpha & 0 & \cos \alpha
\end{array}\right] \cdot \mathbf{K}_{\mathrm{dyn}-\mathrm{h}}^{3 \mathrm{D}}(\omega) \cdot\left[\begin{array}{ccc}
\cos \alpha & 0 & \sin \alpha \\
0 & 1 & 0 \\
-\sin \alpha & 0 & \cos \alpha
\end{array}\right]^{-1}
$$

where $\mathbf{K}_{\text {dyn-h }}^{3 \mathrm{D}}(\omega)$ is defined by eq. (2.35) in section 2.2.2.

\subsection{A numerical example}

In this section, a numerical example is given to illustrate how to analyze the static and dynamic characteristics of an inclined sagging cable by using the introduced cable model. The studied cable is a kind of anti-rust steel made by CarlStahl ${ }^{1}$ which is often used as the driving cables of CDPRs. The basic cable parameters are given in table 2.1.

\subsubsection{Static cable characteristics}

According to the static cable model introduced in section 2.1.1, for a given static external force applied on the end point of the example cable, the static cable profile can be obtained by substituting the basic cable parameters (see table 2.1) and the external forces to eqs. (2.9)

\footnotetext{
${ }^{1}$ http://www.carlstahl-architektur.com/
} 
Table 2.1 Physical parameters of the studied cable

\begin{tabular}{l|l}
\hline Parameter & Value \\
\hline Young's modulus $E$ & $20 \mathrm{Gpa}$ \\
Diameter $D$ & $4 \times 10^{-3} \mathrm{~m}$ \\
Unstrained cross-sectional area $A$ & $1.26 \times 10^{-5} \mathrm{~m}^{2}$ \\
Unstrained cable length $l_{u s}$ & $20 \mathrm{~m}$ \\
Cable mass per meter $\rho$ & $0.067 \mathrm{~kg} / \mathrm{m}$ \\
Damping parameter $\xi$ & 0.01 \\
\hline
\end{tabular}

and (2.10). Three groups of external forces are examined here. The corresponding static cable profiles are represented in fig. 2.4, where the solid lines represent the cable profiles and the dotted lines represent the chords of the sagging curves. Static cable parameters of the example cable under the three groups of external forces are computed respectively according to eqs. (2.11) (2.22). Results are listed in table 2.2.

Table 2.2 Static cable parameters of the example cable under different external forces

\begin{tabular}{|l|c|c|c|}
\hline External forces $\left[f_{C x} f_{C z}\right]^{\mathrm{T}}(\mathrm{N})$ & {$[1010]^{\mathrm{T}}$} & {$[4040]^{\mathrm{T}}$} & {$[100100]^{\mathrm{T}}$} \\
\hline \hline End-point coordinate $\left(x_{C} z_{C}\right)(\mathrm{m})$ & $(10.59-16.83)$ & $(13.06-15.13)$ & $(13.70-14.58)$ \\
\hline Inclined angle $\alpha\left(^{\circ}\right)$ & 57.8 & 49.2 & 46.8 \\
\hline Chord length $l_{c}(\mathrm{~m})$ & 19.89 & 19.99 & 20.01 \\
\hline Elongation $\Delta l(\mathrm{~m})$ & 0.0015 & 0.0049 & 0.0116 \\
\hline Cable sag $d(\mathrm{~m})$ & 0.912 & 0.347 & 0.153 \\
\hline
\end{tabular}

As we can see from fig. 2.4 and table 2.2, an obvious fact can be found that the bigger the external forces are, the smaller the cable sag becomes. In addition, the inclined angle decreases with the growing of the external forces, and it will tend to be $45^{\circ}$ (because $\left|f_{C x}\right|$ and $\left|f_{C z}\right|$ are the same here) when the external forces tend to be infinite. Moreover, it is shown that the chord length is shorter than the initial cable length for small external forces, such as $\left[\begin{array}{ll}10 & 10\end{array}\right]^{\mathrm{T}} \mathrm{N}$. Because in this situation, the cable sag is obvious and the cable elongation is negligible. While the chord length is almost the same as the initial cable length if the external forces grow to $[4040]^{\mathrm{T}} \mathrm{N}$. Because in this case, the cable sag decreases and the cable elongation increases. When the external forces research $\left[\begin{array}{lll}100 & 100\end{array}\right]^{\mathrm{T}} \mathrm{N}$, the chord length is even longer than the initial cable length. This means that the cable elongation under the effect of external forces is non-negligible and the cable sag is negligible. To sum up, for a given cable, the external forces determine the relationship of the cable sag and the cable elongation, thus affect the length of cable chord.

In order to study the static behavior of the example cable further, the static compliance 


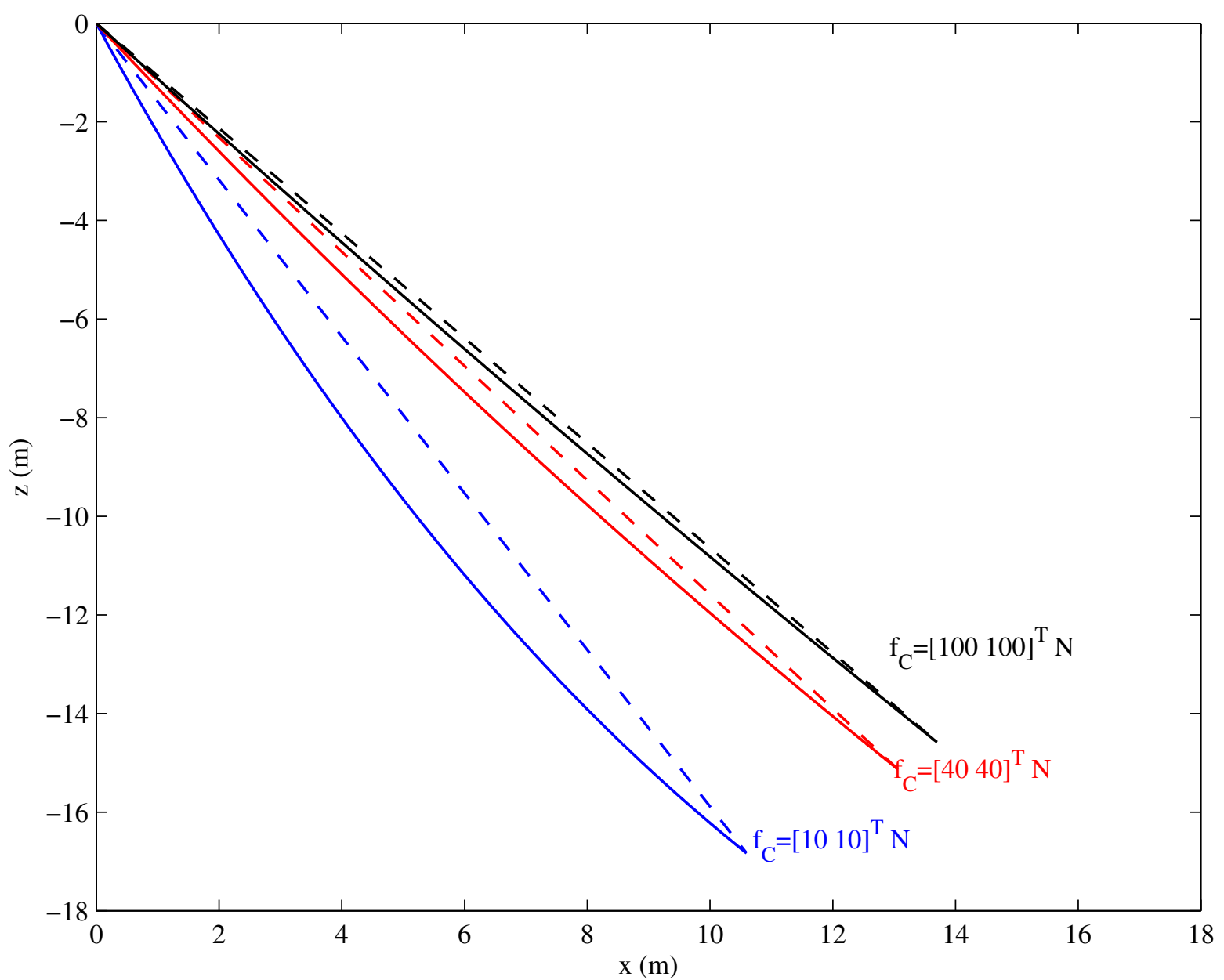

Fig. 2.4 Profiles of the example cable under different external forces: the solid lines and the dashed lines represent the cable profiles and the cable chords, respectively

Table 2.3 Static compliance and stiffness matrix of the example cable under different external forces

\begin{tabular}{|l|c|c|c|c|}
\hline$\left[f_{C x} f_{C z}\right]^{\mathrm{T}}(\mathrm{N})$ & {$[1010]^{\mathrm{T}}$} & {$[4040]^{\mathrm{T}}$} & {$[100100]^{\mathrm{T}}$} \\
\hline \hline $\mathbf{C}_{\text {axial }}^{2 \mathrm{D}}\left(10^{-4} \mathrm{~m} / \mathrm{N}\right)$ & {$\left[\begin{array}{cc}0.7958 & 0 \\
0 & 0.7958\end{array}\right]$} & {$\left[\begin{array}{cc}0.7958 & 0 \\
0 & 0.7958\end{array}\right]$} & {$\left[\begin{array}{cc}0.7958 & 0 \\
0 & 0.7958\end{array}\right]$} \\
\hline $\mathbf{C}_{\text {sag }}^{2 \mathrm{D}}(\mathrm{m} / \mathrm{N})$ & {$\left[\begin{array}{cc}0.7367 & 0.4741 \\
0.4741 & 0.3227\end{array}\right]$} & {$\left[\begin{array}{cc}0.1864 & 0.1610 \\
0.1610 & 0.1401\end{array}\right]$} & {$\left[\begin{array}{cc}0.0727 & 0.0683 \\
0.0683 & 0.0642\end{array}\right]$} \\
\hline $\mathbf{K}_{\text {static }}^{2 \mathrm{D}}(\mathrm{N} / \mathrm{m})$ & {$\left[\begin{array}{cc}24.78 & -36.40 \\
-36.40 & 56.57\end{array}\right]$} & {$\left[\begin{array}{cc}709.8 & -815.7 \\
-815.7 & 944.5\end{array}\right]$} & {$\left[\begin{array}{cc}3846 & -4083 \\
-4083 & 4350\end{array}\right]$} \\
\hline
\end{tabular}

matrix and the stiffness matrix of the example cable under different external forces are computed respectively according to eqs.(2.25) (2.28). Results are listed in table 2.3. Firstly, 
it is shown that the compliance matrix caused by the axial cable elasticity $\mathbf{C}_{\mathrm{axial}}^{2 \mathrm{D}}$ are the same although the external forces are quite different. This can be explained by the definition of $\mathbf{C}_{\text {axial }}^{2 \mathrm{D}}: L_{u s}, E, A$ are the same for a given cable (see eq. (2.28)). Secondly, the compliance caused by the sag-introduced flexibility $\mathbf{C}_{\text {sag }}^{2 \mathrm{D}}$ becomes much smaller when the external forces grow larger. Compared with the axial cable elasticity, the sag-introduced flexibility is much more important even if the external forces research $\left[\begin{array}{lll}100 & 100\end{array}\right]^{\mathrm{T}}$. Finally, we can see that the stiffness matrix $\mathbf{K}_{\text {static }}^{2 \mathrm{D}}$ increases with the growing of external forces. To sum up, the compliance of a sagging cable is caused by the axial cable elasticity and the sag-introduced flexibility. The axial cable elasticity is only related to the physical cable parameter, while the sag-introduced flexibility is mainly determined by the external forces. In this example, the cable stiffness is mainly affected by the sag-introduced flexibility.

\subsubsection{Dynamic cable characteristics}

As we explained in section 2.2.3, the proposed dynamic model of an inclined sagging cable is valid when the cable parameters $\lambda^{2}$ and $\varepsilon$ together with the inclined angle $\alpha$ satisfy the limits defined by eqs. (2.40) and (2.41). Therefore, before the analysis of dynamic cable characteristics, these three parameters are firstly computed to check the limits. Results are listed in table 2.4 .

Table 2.4 Dynamic cable parameters of the example cable under different external forces

\begin{tabular}{|c|c|c|c|}
\hline$\left[f_{C x} f_{C z}\right]^{\mathrm{T}}(\mathrm{N})$ & {$\left[\begin{array}{ll}10 & 10\end{array}\right]^{\mathrm{T}}$} & {$\left[\begin{array}{ll}40 & 40\end{array}\right]^{\mathrm{T}}$} & {$\left[\begin{array}{lll}100 & 100\end{array}\right]^{\mathrm{T}}$} \\
\hline$\lambda^{2} \lambda^{2}$ & 1778.5 & 79.1 & 6.4 \\
\hline$\alpha$ & $57.8^{\circ}$ & $49.2^{\circ}$ & $46.8^{\circ}$ \\
\hline$\varepsilon$ & 0.367 & 0.139 & 0.061 \\
\hline
\end{tabular}

As we can see from table 2.4, among the 3 groups of external forces, the limits are valid only for the third group where the external forces are $\left[\begin{array}{lll}100 & 100\end{array}\right]^{\mathrm{T}} \mathrm{N}$. In fact, the dynamic model introduced from civil engineering [Starossek 1991b] is usually used for the vibration analysis of a taut inclined cable. This dynamic cable model is not suitable for slack cable with big sag. Nevertheless, it is not a problem for most applications of CDPRs because CDPRs are often designed to avoid cable slackness. The significance of this dynamic model lies in its ability to analyze cable dynamics with considering not only the cable elasticity but also the effect of cable mass on the cable dynamics. In the following chapters, we will see the important effect of cable dynamics on the dynamic behavior of CDPRs.

The dynamic stiffness matrix $\mathbf{K}_{\text {dyn }}^{3 \mathrm{D}}(\omega)$ of the example cable under the external forces $[100100]^{\mathrm{T}} \mathrm{N}$ is calculated according to eqs. (2.31), (2.35) and (2.45). Then the dynamic 
stiffness matrix is diagonalized, and the trace of the diagonal matrix is computed. For comparison, same calculations are made with the static stiffness matrix of the example cable under the equal external forces.

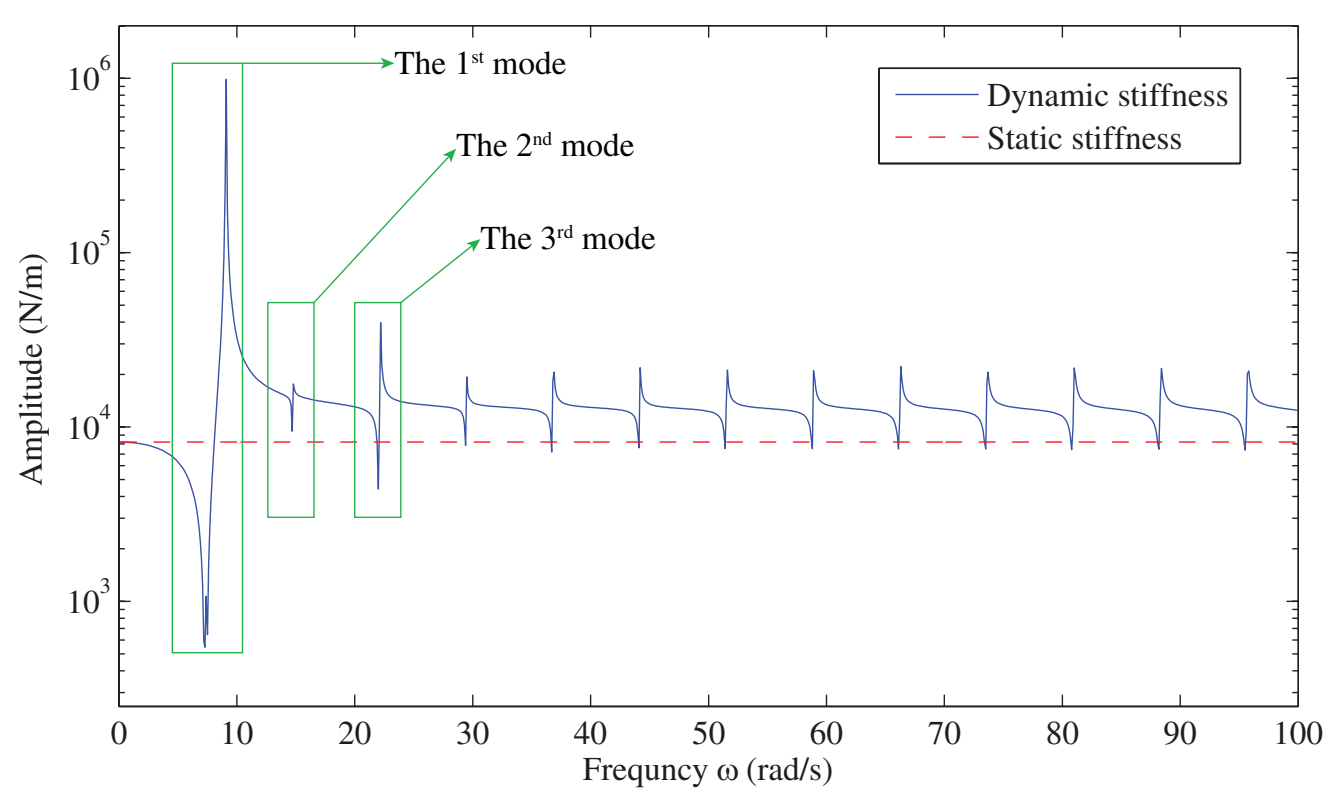

Fig. 2.5 Amplitude variation of the trace of the dynamic and static stiffness matrix for the example cable

The amplitude variations of the trace of the dynamic and static stiffness matrix are plotted with respect to the frequency of harmonic motion $\omega$ in fig. 2.5. As expected, the trace of the static stiffness matrix is constant, and the trace of the dynamic stiffness matrix is variable with frequency. Considerable variations are present within the examined frequency, and these variations are associated with symmetric and antisymmetric modes of the cable. For example, the first resonance corresponds the fundamental frequency of the example cable. According to [Irvine 1992], the first peak here is related to the first out-of-plane mode, and the corresponding natural frequency can be calculated by $\Omega=\pi \sqrt{\frac{\tau}{\rho g l_{c}}}$. The computed natural frequency coincides with the natural frequency identified from fig. 2.5. By using the DSM method, the cable vibration is introduced by a variation of its own stiffness matrix through the frequency range. 


\subsection{Summary of the chapter}

This chapter addresses on the static and dynamic cable modeling, which is the basis of the CDPR modeling in the following chapter. The static sagging cable model and the dynamic DSM method are introduced in details respectively.

A significant feature of the cable modeling in this chapter is the consideration of the cable elasticity and the effect of cable weight on the static cable profile. Thus, compared with previous cable models used in CDPR analysis, the models in this chapter are more accurate. However accurate cable modeling also results in increased complexity. For example, nonlinear equations are used to describe the profile of static sagging cable, which leads to the coupling of cable forces and cable lengths. The balance of accuracy and complexity will be further discussed in the following chapter.

Another important result is the introduction of the DSM method in the dynamic cable analysis. With the DSM method, the dynamic characteristics of an inclined sagging cable can be expressed, concisely and effectively by a stiffness matrix. However, this method has a limitation. It is only applicable for taut cable with small sag. The limits should be verified (see eqs. (2.40) and (2.41)) before the use of DSM method. In fact, these limits are not a problem for most applications of CDPRs. Because CDPRs are often designed to avoid cable slackness, and driving cables are often working in enough tension. In spite of this, the significance of DSM cable model lies in its ability to relate cable dynamics with CDPR dynamics. In the following chapters, we will see the important effect of cable dynamics on the dynamic performances of CDPRs. 


\section{Chapter 3}

\section{Static and Dynamic Stiffness Modeling of CDPRs}

\section{Contents}

3.1 Problem description $\ldots \ldots \ldots \ldots \ldots \ldots \ldots \ldots$

3.2 Static stiffness modeling of CDPRs . . . . . . . . . . . . . 37

3.3 Dynamic stiffness modeling of CDPRs . . . . . . . . . . 42

3.4 A numerical Example . . . . . . . . . . . . . . . 48

3.5 Summary of the chapter $\ldots \ldots \ldots \ldots \ldots \ldots$

The static and dynamic cable models with non-negligible cable mass and elasticity have been presented in the previous chapter. Based on the cable models, this chapter will address on the static and dynamic stiffness modeling of CDPRs.

One major purpose of static stiffness modeling of CDPRs is to study their static positioning accuracy. As is known, the static stiffness of CDPRs and the static positioning accuracy are closely associated with each other. In this thesis, we use the variation of the pose error with external load to study the static stiffness of CDPRs. In this chapter, the kinematic model of CDPRs is firstly presented considering both the cable elasticity and the effect of cable weight on the static cable profile. Then the static pose error of the end-effector is defined and calculated based on the direct kinematic model of CDPRs. After that, the variation of the pose error with external load is used as an index to evaluate the static stiffness of CDPRs.

As explained in Chapter 1, an important issue of dynamic analysis of CDPRs is to find out whether the cable resonances and cable vibrations affect the dynamics of CDPRs. In other words, what is the limit of the assumption of a massless cable modeling in the complete 
dynamic CDPR model? To achieve this purpose, a new dynamic stiffness model of CDPRs is proposed. This model considers the rigid-body vibration of the end-effector suspended on the cable stiffness, the own cable vibration and the coupling between both. It is a complete model to describe the dynamics of CDPRs. Through this dynamic model, the natural frequencies of CDPRs can be identified and the coupling of cable dynamics and end-effector vibrations can be analyzed. In this chapter, the dynamic stiffness matrix of CDPRs is formulated and the oscillation model of the end-effector is set up through the Lagrange's equations. After that, the dynamic response functions under a harmonic excitation are calculated and system natural frequencies can be identified from the FRF plots. At the end of this chapter, an example is given to illustrate how to use the proposed methods on the stiffness analysis of CDPRs.

\subsection{Problem description}

It should be noted that the static stiffness analysis in this chapter mainly focuses on suspended CDPRs. The reasons are as following: for non-suspended CDPRs, sag-introduced flexibility can be reduced by increasing internal cable forces. As long as the internal cable forces are big enough, the effect of cable sag can be neglected, and the assumption of massless linear spring cable can be used in the static stiffness analysis. However, the method of increasing internal forces cannot be applied to suspended CDPRs. Because there does not exist a cable below the end-effector, and the cable forces mainly depend on the external load applied to the end-effector. Therefore, sag-introduced stiffness should be considered in the stiffness analysis of CDPRs, especially when the external load is small.

Although suspended CDPRs are mainly presented, the proposed method can be applied and be useful in the analysis of non-suspended CDPRs. In fact, increasing internal cable forces is a passive method, which directly leads to the augmentation of motor power and energy consumption. In practical applications, it is impossible to eliminate sag-introduced flexibility entirely, especially for the CDPRs with heavy and long-span cables. Therefore, the proposed method is also significant for the stiffness analysis of non-suspended CDPRs. The application of the proposed models on non-suspended CDPRs will be further presented in Chapter 5. Figure 3.1 presents the schematic diagram of a suspended CDPR, where:

- $A_{i}$ and $B_{i}$ represent the attachment points on the end-effector and on the fixed base, respectively; $L_{i}$ presents the $i^{\text {th }}$ cable;

- $\mathfrak{R}_{G}\left(O_{G}, x_{G}, y_{G}, z_{G}\right)$ represents the global frame; 
- $\mathfrak{R}_{e}\left(O_{e}, x_{e}, y_{e}, z_{e}\right)$ represents the local frame fixed on the end-effector;

- $\mathfrak{R}_{B_{i}}\left(B_{i}, X, Y, Z\right)$ represents an auxiliary frame, whose origin point is $B_{i}$, and whose axises are parallel to the axises of the global frame $\mathfrak{R}_{G}\left(O_{G}, x_{G}, y_{G}, z_{G}\right)$;

- $\mathfrak{R}_{c i}\left(O_{c i}, x_{c i}, y_{c i}, z_{c i}\right)$ represents the local cable frame, where the origin point $O_{c i}$ is coincident with $B_{i}$, axis $z_{c i}$ is parallel to $z_{G}$, axis $x_{c i}$ is in the cable plane.

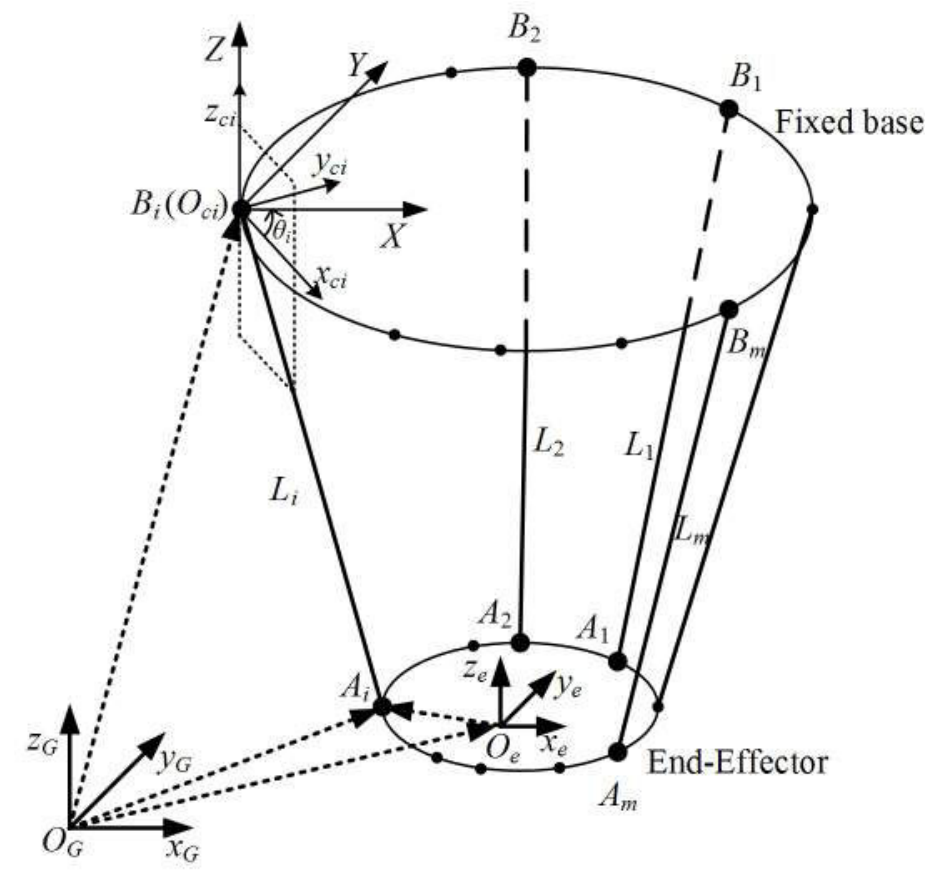

Global coordinate

Fig. 3.1 The schematic diagram of a suspended CDPR

\subsection{Static stiffness modeling of CDPRs}

In this section, both the cable elasticity and the effect of cable weight on the cable profile are considered in the static stiffness modeling of CDPRs. The inverse kinematic model and the direct kinematic model of CDPRs are presented. Discussions of the solution of the kinematic model are made based on the relationship between the number of driving cables and the number of DOFs. Then the static pose error of the end-effector is defined through the direct kinematic model of CDPRs. After that, the variation of the end-effector pose error with the external load is defined as an index for the static stiffness evaluation of CDPRs. 


\subsubsection{Kinematic modeling}

\section{a) Inverse kinematic modeling}

The objective of the inverse kinematic model of CDPRs is to calculate the length of all the driving cables for a given pose of the end-effector. If the cable elasticity and the effect of cable weight on the static cable profile are both considered, the inverse kinematic model is coupled with the static equilibrium, which means that the cable lengths and the cable forces must be calculated at the same time. The general procedure of solving inverse kinematics is as following:

\section{(1) Equations from the geometric relationship}

The coordinate of $A_{i}$ in the global frame $\mathfrak{R}_{G}$ is:

$$
G \overrightarrow{O_{G} A_{i}}=G \overrightarrow{O_{G} O_{e}}+{ }^{G} \overrightarrow{O_{e} A_{i}}={ }^{G} \overrightarrow{O_{G} O_{e}}+{ }^{G} \mathbf{T}_{e}{ }^{e} \overrightarrow{O_{e} A_{i}}
$$

where ${ }^{G} \mathbf{T}_{e}$ is the rotation matrix that transfers the coordinates in $\Re_{e}$ to their corresponding coordinates in $\Re_{G}$.

The coordinate of $A_{i}$ in the cable frame $\mathfrak{R}_{c i}$ is:

$$
{ }^{c i} \overrightarrow{O_{c i} A_{i}}={ }^{G} \mathbf{T}_{c i}^{-1}{ }^{G} \overrightarrow{O_{c i} A_{i}}={ }^{G} \mathbf{T}_{c i}^{-1}\left({ }^{G} \overrightarrow{O_{G} A_{i}}-{ }^{G} \overrightarrow{O_{G} O_{c i}}\right)
$$

where ${ }^{G} \mathbf{T}_{c i}$ is the rotation matrix that transfers the coordinates in $\Re_{c i}$ to their corresponding coordinates in $\Re_{G}$, and the rotation angle can be computed as $\theta_{i}=\arctan \left(\frac{y_{\overrightarrow{A_{i} B_{i}}}}{\vec{x}_{\bar{A}_{i} B_{i}}}\right)$, where $y_{\overrightarrow{A_{i} B_{i}}}$ and $x_{A_{i} B_{i}}$ are the components of vector $\overrightarrow{A_{i} B_{i}}$ expressed in the frame $\Re_{B_{i}}$.

(2) Equations from the static sagging cable model

The coordinates of $A_{i}$ in the cable frame $\mathfrak{R}_{c i}$ can also be obtained by substituting $s=l_{u s i}$ into eqs. (2.9) and (2.10):

$$
\begin{aligned}
& x_{\overrightarrow{O_{c i} A_{i}}}=x\left(l_{u s i}\right)=\frac{f_{A x i} l_{u s i}}{E A}+\frac{\left|f_{A x i}\right|}{\rho g}\left[\sinh ^{-1}\left(\frac{f_{A z i}}{f_{A x i}}\right)-\sinh ^{-1}\left(\frac{f_{A z i}-\rho g l_{u s i}}{f_{A x i}}\right)\right], \\
& z_{\overrightarrow{O_{c i} A_{i}}}=z\left(l_{u s i}\right)=\frac{f_{A z i} l_{u s i}}{E A}-\frac{\rho g l_{u s i}^{2}}{2 E A}+\frac{1}{\rho g}\left[\sqrt{f_{A x i}^{2}+f_{A z i}^{2}}-\sqrt{f_{A x i}^{2}+\left(f_{A z i}-\rho g l_{u s i}\right)^{2}}\right],
\end{aligned}
$$

where $i=1,2, \cdots m$. For a CDPR driven by $m$ cables, the number of equations from the static cable model is $2 m$.

(3) Equations from the static equilibrium of the end-effector 
The equations for the static equilibrium of the end-effector can be expressed as:

$$
\begin{array}{r}
\sum_{i=1}^{m}{ }^{G} \mathbf{f}_{A i}+{ }^{G} \mathbf{f}_{e x}=\mathbf{0}, \\
\sum_{i=1}^{m}\left({ }^{G} \overrightarrow{O_{e} A_{i}} \times{ }^{G} \mathbf{f}_{A i}\right)+{ }^{G} \mathbf{m}_{e x}=\mathbf{0},
\end{array}
$$

where ${ }^{G} \mathbf{f}_{e x}$ and ${ }^{G} \mathbf{m}_{e x}$ are respectively the external forces and moments expressed in the global frame, and ${ }^{G} \mathbf{m}_{e x}$ is calculated with respect to the original point of the cable frame $\mathfrak{R}_{e} \cdot{ }^{G} \mathbf{f}_{A i}$ are the forces exerted by the cables on the end-effector at points $A_{i}$, and ${ }^{G} \mathbf{f}_{A i}={ }^{G} \mathbf{T}_{c i}{ }^{c i} \mathbf{f}_{A i}$, ${ }^{c i} \mathbf{f}_{A i}=\left[\begin{array}{lll}-f_{A x i} & 0 & -f_{A z i}\end{array}\right]^{T}$. For a CDPR with $n$ DOFs, the number of static equilibrium equations is $n$, where $n \leq 6$.

(4) Solving the equations

Due to the coupling of the cable forces and the cable lengths, there is no analytical solution for the above nonlinear set of equations. Numerical solutions are usually computed. In this section, a constrained nonlinear optimization algorithm is developed through Matlab ${ }^{1}$ to solve eqs. (3.3) to (3.6). In this set of non-linear equations, $l_{u s i}, f_{A x i}, f_{A z i}(i=1,2 \cdots m)$ are the unknown variables. Finally, there are $3 m$ unknowns and $2 m+n$ equations. According to the relationship between the number of driving cables $m$ and the DOFs $n$, three cases should be considered:

1. $m=n$, there are as many unknowns as the equations. Generally there exists a unique solution of the inverse kinematic model.

2. $m<n$, there are more equations than the unknowns, and the solutions may not exist. This means that the end-effector cannot be positioned arbitrarily in the 6 DOFs.

3. $m>n$, the unknowns outnumber the equations, so the solution will not be unique. In this case, different sets of cable lengths and forces can be obtained for the same given pose of the end-effector. To make the solution unique, constrained optimization with a cost function can be used.

\section{(5) Initial guess for the iteration}

A good initial guess is important for the convergence and the efficiency of the optimization method. Assuming cables are ideal straight lines without mass and elasticity, the cable forces and cable lengths are uncoupled. The ideal cable lengths can be calculated through the

\footnotetext{
${ }^{1}$ Matlab is a software of MathWorks ${ }^{\circledR}$ company (http://www.mathworks.com).
} 
inverse kinematic model $\left(l_{0 i}=\left|\overrightarrow{A_{i} B_{i}}\right|\right)$. The ideal cable forces can be calculated through the static equilibrium of the end-effector $\left(\mathbf{J}^{T} \mathbf{f}_{\text {ideal }}+\mathbf{w}_{e x}=\mathbf{0}\right)$, where $\mathbf{J}^{T}$ is the transposition of the Jacobian matrix, $\mathbf{f}_{\text {ideal }}=\left[f_{1} f_{2} \cdots f_{m}\right]^{T}$ is the column vector of the ideal cable forces, and $\mathbf{w}_{e x}$ is the external wrench (force and moment) applied on the end-effector. Then the calculated cable lengths and forces can be used as the initial guess.

During the calculation of cable forces, three cases should be considered according to the rank of $\mathbf{J}^{T}$ :

1. $m=n, \mathbf{J}^{T}$ is full rank and reversible except for the singulary poses. The ideal cable forces can be calculated by $\mathbf{f}_{\text {ideal }}=-\mathbf{J}^{-T} \mathbf{w}_{e x}$.

2. $m<n, \mathbf{J}^{T}$ is not full rank. There are more equations than variables in $\mathbf{J}^{T} \mathbf{f}_{\text {ideal }}+\mathbf{w}_{e x}=\mathbf{0}$. The ideal cable forces $\mathbf{f}_{\text {ideal }}$ can be calculated by choosing any $m$ equations from the $n$ equations. Then the solution should be substituted back into the remaining $n-m$ equations to be tested.

3. $m>n, \mathbf{J}^{T}$ is not full rank. There are less equations than variables in $\mathbf{J}^{T} \mathbf{f}_{\text {ideal }}+\mathbf{w}_{e x}=\mathbf{0}$. Different force distribution methods [Pott+ 2009; Mikelsons+ 2008; Gosselin+ 2011] can be used to calculate the ideal cable forces.

\section{b) Direct kinematic modeling}

The objective of the direct kinematic model is to calculate the pose of the end-effector for a given set of cable lengths. The constraints of the direct kinematic model are the same with that of the inverse kinematic model (eqs. (3.3) to (3.6)). If the effect of cable weight on the static cable profile is non-negligible, the direct kinematic model of CDPRs is coupled with the static equilibrium of the end-effector. The end-effector pose and the cable forces should be calculated at the same time. For an $n$ DOFs CDPR with $m$ driving cables, there are $2 m+n$ equations (eqs. (3.3) to (3.6)) and $2 m+n$ unknown variables. Various kinds of methods can be used for solving the direct kinematic problem [Pott 2010; Von Zitzewitz+ 2010; Berti+ 2013]. In this chapter, an optimization method is used to solve the direct kinematic problem. This method will be further detailed in section 3.2.2.

\subsubsection{Static pose error definition}

Assuming that the compliant displacements of the end-effector under external load are small, the pose error of the end-effector can be calculated by its static Cartesian stiffness matrix [Carbone 2011]. However, considering the effect of cable weight on the static cable profile, 
the sag-introduced flexibility should be taken into account. The small compliant displacement assumption may not valid, especially for heavy and/or long cables with relatively light endeffector. Therefore, the Cartesian stiffness matrix cannot be used to calculate the pose error. In this thesis, the direct kinematic model of CDPRs formulated in section 3.2.1 is used to define and compute the pose error of the end-effector.

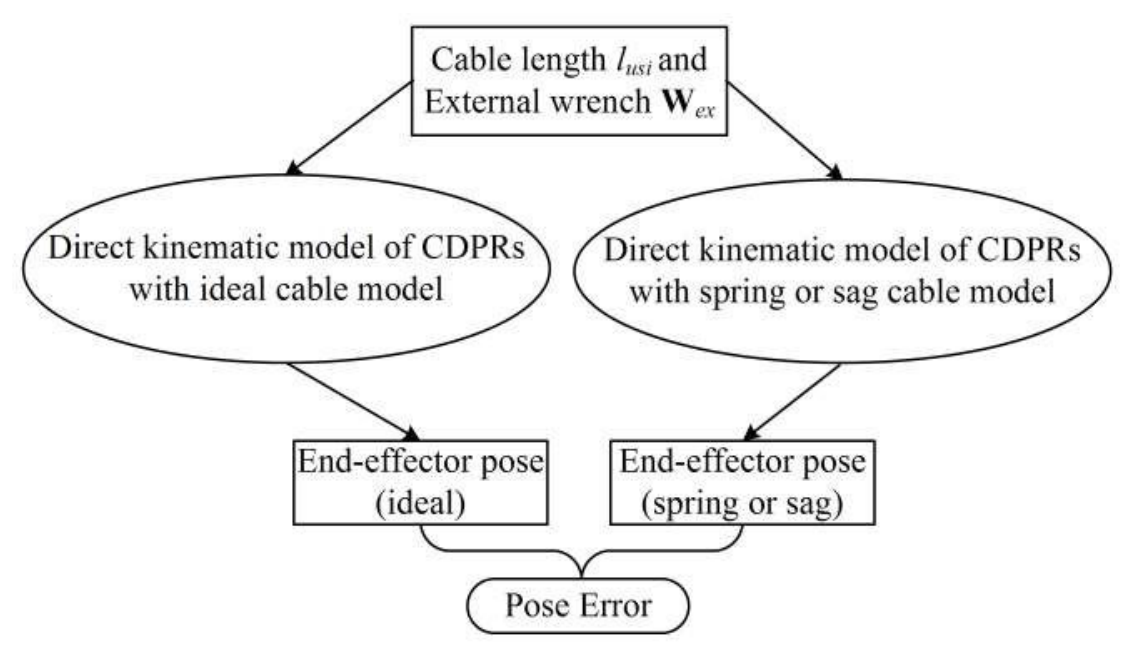

Fig. 3.2 Definition of the static pose error

Fig. 3.2 presents the definition of the pose error of the end-effector. For a given set of unstrained cable lengths $l_{u s i}$, the pose (position and orientation) of the end-effector can be obtained through the direct kinematic model. In the direct kinematic modeling, different cable models can be used, such as the ideal cable model where the cable is considered to be an inextensible straight line, the spring cable model where the cable is simplified as linear spring without mass, and the sagging cable model where the elastic catenary is employed considering both the cable elasticity and the effect of cable weight on the static cable profile. The difference between the pose obtained through the spring cable model or the sagging cable model and the reference pose obtained through the ideal cable model defines the static pose error of the robot. It should be noted that the cable lengths $l_{u s i}$ are not given arbitrarily. In fact, it is a two step procedure. Firstly, a pose of the end-effector in its workspace is chosen as a reference. Then the reference cable lengths are obtained from the ideal inverse kinematic model, and the set of cable lengths $l_{u s i}$ is given according to the reference lengths. In this way, the reference pose of the end-effector can be used as a good initial guess for the direct kinematic model. 


\subsubsection{Static stiffness evaluation}

The variation of the end-effector pose error is regarded as the index for static stiffness evaluation. First of all, this method is simple. It has a direct natural interpretation as it is associated with the compliant displacement of the end-effector under the effect of external load. Secondly, the stiffness along different directions can be evaluated by the pose error along the corresponding direction. We can focus on the stiffness along certain interesting direction. Taking the pick-and-place application of suspended CDPRs for example, the stiffness along vertical direction should be paid more attention to, as the stiffness along this direction is more important to ensure a good position accuracy. Thirdly, considering the cable elasticity and the effect of cable weight on the static cable profile, the static stiffness of CDPRs depends on the external load. The variation of the static stiffness with external load can be presented by the change of the pose error with external load.

Furthermore, the static stiffness of CDPRs has two sources: sag-introduced flexibility and axial cable elasticity. The variation of the end-effector pose error calculated by the sagging cable model presents the global system stiffness. While the variation of the end-effector pose error calculated by the spring cable model only reflects the contribution of axial cable elasticity to the system stiffness. The difference between the results obtained by sagging cable model and the results by spring cable model expresses the contribution of sag-introduced flexibility. Thus, the effect of each source on the system stiffness can be clearly defined. In addition, pose error is easy to measure, which is convenient for experimental validation. It should be noticed that stiffness matrix and its mathematical properties are also powerful for stiffness analysis (as illustrated in [Arsenault 2013]). In this thesis, these two methods are not compared with each other.

\subsection{Dynamic stiffness modeling of CDPRs}

The main purpose of the dynamic stiffness modeling of CDPRs is to analyze the effect of cable dynamic on the dynamic behavior of CDPRs. To be more precise, it aims to answer the question that whether the effect of cable vibration has a significant influence towards the system dynamics of CDPRs. Besides, another objective of dynamic stiffness modeling of CDPRs is to identify the natural frequencies of CDPRs. Because natural frequencies, especially the fundamental frequency is a useful tool to evaluate the global stiffness and the vibration of CDPRs.

In this section, the dynamic stiffness matrix of CDPRs is firstly formulated. This dynamic 
matrix is an assemblage of the dynamic stiffness matrix of all the driving cables (refer to section 2.2). It considers the cable elasticity and the effect of cable mass on the cable dynamics. With this dynamic stiffness matrix, the oscillating equations of the end-effector around a static equilibrium are formulated through the Lagrange's equations. Then dynamic response functions under a harmonic excitation are calculated. All the dynamic informations of CDPRs are contained in the dynamic response functions, such as the vibration of each driving cable, the vibration of the end-effector and their coupling. Through the plots of the dynamic response functions, the natural frequencies of CDPRs can be identified and the effect of cable dynamics on the system dynamic behavior can be analyzed.

\subsubsection{Computation of the dynamic stiffness matrix of CDPRs}

For CDPRs, the system stiffness is mainly affected by the stiffness of their cables, actuators and end-effector. Compared with cables, the compliance of the actuators and the end-effector is much lower and therefore can be neglected. Thus the dynamic stiffness of CDPRs can be formulated by assembling the dynamic stiffness matrices of all the driven cables.

In section 2.2, the dynamic stiffness matrix of an inclined sagging cable ${ }^{c i} \mathbf{K}_{i}(\omega)$ (in eq. (2.46) it is written as $\mathbf{K}_{\mathrm{dyn}}^{3 \mathrm{D}}$ ) is expressed in the cable frame $\mathfrak{R}_{c i}$. In order to assemble the dynamic stiffness matrices of all the driving cables together to formulate the system dynamic matrix, it is necessary to transform ${ }^{c i} \mathbf{K}_{i}(\omega)$ to the global frame $\mathfrak{R}_{G}$.

$$
{ }^{G} \mathbf{K}_{i}(\omega)={ }^{G} \mathbf{T}_{c i}{ }^{c i} \mathbf{K}_{i}(\omega){ }^{G} \mathbf{T}_{c i}^{-1}
$$

where ${ }^{G} \mathbf{T}_{c i}$ is the rotation matrix. Then the stiffness matrix of the robot ${ }^{G} \mathbf{K}_{E}(\omega)$ can be formulated by assembling the dynamic stiffness matrix of all the driven cables:

$$
\begin{aligned}
{ }^{G} \mathbf{K}_{E}(\omega) & =\sum_{i=1}^{m} \mathbf{A}_{i}^{T G} \mathbf{K}_{i}(\omega) \mathbf{A}_{i}, \\
\text { with: } \quad \mathbf{A}_{i} & =\left[\begin{array}{cccccc}
1 & 0 & 0 & 0 & -z \overrightarrow{o_{e} A_{i}} & y \overrightarrow{o_{e} A_{i}} \\
0 & 1 & 0 & z \overrightarrow{o_{e} A_{i}} & 0 & -x \overrightarrow{o_{e} A_{i}} \\
0 & 0 & 1 & -y \overrightarrow{o_{e} A_{i}} & x_{\overrightarrow{o_{e} A_{i}}} & 0
\end{array}\right],
\end{aligned}
$$

where $x \underset{o_{e} A_{i}}{\longrightarrow}, y \underset{o_{e} A_{i}}{\longrightarrow}$ and $z_{o_{e} A_{i}}$ are the components of the vector $\overrightarrow{o_{e} A_{i}}$ along axis- $x_{G}, y_{G}$ and $z_{G}$ expressed in the global frame $\mathfrak{R}_{G}$. In addition, the dynamic stiffness matrix of CDPRs can 
also be expressed in the end-effector frame $\mathfrak{R}_{e}$ through the rotation matrix ${ }^{G} \mathbf{T}_{e}$ :

$$
{ }^{e} \mathbf{K}_{E}(\omega)={ }^{G} \mathbf{T}_{e}^{-1}{ }^{G} \mathbf{K}_{E}(\omega){ }^{G} \mathbf{T}_{e}
$$

As explained in section 2.2, the dynamic stiffness matrix of an inclined sagging cable is frequency-dependent because it considers the effect of cable mass on the cable dynamics. As an assemblage of the dynamic stiffness matrices of all the driving cables, the dynamic stiffness matrix of CDPRs are also frequency-dependent, which means that each element of ${ }^{G} \mathbf{K}_{E}(\omega)$ is a function of frequency $\omega$. This dynamic stiffness matrix of CDPRs contains all the dynamic characteristics of the driving cables, which will be used in the following dynamic modeling of CDPRs.

\subsubsection{Oscillating model of the end-effector around a static equilibrium}

The stiffness of CDPRs is mainly affected by the stiffness of the driving cables, the actuators and the end-effector. Compared with cables, the compliance of the actuators and the endeffector is much lower and therefore can be neglected. In the oscillating modeling, the end-effector is considered as a rigid-body suspended on the stiffness of the cables, and then its dynamic pose around its static equilibrium can be defined by 6 DOFs ( 3 translations and 3 rotations). These 6 motions of the end-effector can be defined as the generalized coordinates of the robot system. The oscillating model of the end-effector around a static equilibrium can be derived in terms of the generalized coordinates by using the Lagrange's equations [Rao+ 1995]:

$$
\frac{d}{d t}\left(\frac{\partial T}{\partial \dot{q}_{i}}\right)-\frac{\partial T}{\partial q_{i}}+\frac{\partial V}{\partial q_{i}}=F_{i}, \quad i=1,2, \cdots 6
$$

where:

- $T$ and $V$ represent the kinetic and potential energies of the system respectively;

- $q_{i}$ represents the generalized coordinates corresponding to the 3 translational motions along axis- $x_{e}, y_{e}$ and $z_{e}$ and the 3 rotational motions around axis- $x_{e}, y_{e}$ and $z_{e}$;

- $\dot{q}_{i}$ represents the time derivatives of the generalized coordinates;

- $F_{i}$ represents the nonconservative generalized forces and moments applied to the end-effector. 
According to the oscillating theory [Bellet 1988], the movements of the end-effector around its static equilibrium can be assumed as small motions, and the Coriolis acceleration can be neglected. Consequently, the oscillating model of the end-effector can be linearized by simplifying the kinetic energy $T$ of the system:

$$
T=\frac{1}{2} \dot{\mathbf{q}}^{T e} \mathbf{M} \dot{\mathbf{q}}
$$

where ${ }^{e} \mathbf{M}$ is the 6 by 6 mass matrix of the end-effector expressed in frame $\Re_{e} ; \mathbf{q}$ and $\dot{\mathbf{q}}$ are the column vectors of the generalized coordinates and their time derivatives: $\mathbf{q}=$ $\left[\begin{array}{llllll}q_{1} & q_{2} & q_{3} & q_{4} & q_{5} & q_{6}\end{array}\right]^{T}$ and $\dot{\mathbf{q}}=\left[\begin{array}{llllll}\dot{q}_{1} & \dot{q}_{2} & \dot{q}_{3} & \dot{q}_{4} & \dot{q}_{5} & \dot{q}_{6}\end{array}\right]^{T}$.

The potential energy $V$ of the system is:

$$
V=\frac{1}{2} \mathbf{q}^{T e} \mathbf{K}_{E} \mathbf{q}
$$

Because the mass matrix ${ }^{e} \mathbf{M}$ is symmetric, we can obtain:

$$
\frac{\partial T}{\partial \dot{q}_{i}}=\frac{1}{2} \delta^{T e} \mathbf{M} \dot{\mathbf{q}}+\frac{1}{2} \dot{\mathbf{q}}^{T}{ }^{e} \mathbf{M} \delta=\delta^{T e}{ }^{e} \dot{\mathbf{q}}=\mathbf{m}_{i}^{T} \dot{\mathbf{q}}
$$

where $\delta_{j i}$ is the Kronecker delta $\left(\delta_{j i}=1\right.$ if $j=i$ and $\delta_{j i}=0$ if $j \neq i$ ), $\delta$ is the column vector of Kronecker deltas whose elements in the rows for which $j \neq i$ are equal to zero and whose element in the row $j=i$ is equal to 1 , and $\mathbf{m}_{i}$ is a column vector which is identical to the $i_{t h}$ column of the mass matrix ${ }^{e} \mathbf{M}$. Due to the mass matrix ${ }^{e} \mathbf{M}$ is constant and independent of time, differentiation of eq. (3.15) with respect to time gives:

$$
\frac{d}{d t}\left(\frac{\partial T}{\partial \dot{q}_{i}}\right)=\mathbf{m}_{i}^{T} \ddot{\mathbf{q}}
$$

where $\ddot{\mathbf{q}}$ is the time derivatives of $\dot{\mathbf{q}}$. The kinetic energy $T$ is a function of only the velocities $\dot{q}_{i}$, thus:

$$
\frac{\partial T}{\partial q_{i}}=0
$$


Like the mass matrix ${ }^{e} \mathbf{M}$, the stiffness matrix ${ }^{e} \mathbf{K}_{E}$ is also symmetric. The partial differentiation of the potential energy can be written as:

$$
\frac{\partial V}{\partial q_{i}}=\frac{1}{2} \delta^{T}{ }^{e} \mathbf{K}_{E} \dot{\mathbf{q}}+\frac{1}{2} \dot{\mathbf{q}}^{T}{ }^{e} \mathbf{K}_{E} \delta=\delta^{T}{ }^{e} \mathbf{K}_{E} \dot{\mathbf{q}}={ }^{e} \mathbf{K}_{E i}^{T} \dot{\mathbf{q}}
$$

where ${ }^{e} \mathbf{K}_{E i}$ is a column vector identical to the $i_{t h}$ column of the stiffness matrix ${ }^{e} \mathbf{K}_{E}$. Substituting eq. (3.16) into eq. (3.11), the dynamic equations of CDPRs in matrix form can be written as:

$$
{ }^{e} \mathbf{M} \ddot{\mathbf{q}}+{ }^{e} \mathbf{K}_{E} \mathbf{q}=\mathbf{w},
$$

where $\mathbf{w}$ is a column vector of the nonconservative forces and moments:

$\mathbf{w}=\left[\begin{array}{llllll}F_{1} & F_{2} & F_{3} & F_{4} & F_{5} & F_{6}\end{array}\right]^{T}$. For the free vibration analysis of CDPRs, there are no nonconservative forces or moments in the oscillating equation of the end-effector around a static equilibrium, which means that $\mathbf{w}=\mathbf{0}$. The free vibration model of the end-effector around a static equilibrium can be written as:

$$
{ }^{e} \mathbf{M} \ddot{\mathbf{q}}(t)+{ }^{e} \mathbf{K}_{E}(\omega) \mathbf{q}(t)=\mathbf{0} .
$$

\subsubsection{Natural frequency identification}

In the previous study [Kozak+ 2006; Diao+ 2009; Ma+ 2005], the effect of cable mass on the cable dynamics is neglected in the vibration analysis. The system stiffness matrix ${ }^{e} \mathbf{K}_{E}$ is constant and independent of the frequency. According to the free vibration theory of multi-degree-of-freedom system, the natural frequencies of the CDPR can be calculated by transforming the system dynamic equation into its modal space, and then solving the classic eigenvalue and eigenvector problems.

However, both the cable elasticity and the effect of cable mass on the cable dynamics are considered in the dynamic stiffness modeling of CDPRs. As a result, the system stiffness matrix ${ }^{e} \mathbf{K}_{E}(\omega)$ that contains cable dynamics is a function of the frequency $\omega$. The above method for multi-degree-of-freedom system is not suitable. The analysis of the dynamic response functions of the robot to a harmonic excitation can be used. For each pose of the end-effector in the workspace, the dynamic equations of a CDPR under harmonic excitations can be written as:

$$
{ }^{e} \mathbf{M} \ddot{\mathbf{q}}(t)+{ }^{e} \mathbf{K}_{E}(\omega) \mathbf{q}(t)=\mathbf{f}(t),
$$

where $\mathbf{f}(t)$ is a column vector of the harmonic excitations and 
$\mathbf{f}(t)=\left[f_{x}(t) f_{y}(t) f_{z}(t) f_{\alpha}(t) f_{\beta}(t) f_{\gamma}(t)\right]^{T}$, which represents the harmonic forces along axis- $x, y$, and $z$ and the harmonic moments around axis- $x, y$, and $z$. According to the vibration theory of linear system, if a harmonic excitation is inputed into the system at a given frequency, the system will respond at that same frequency with a certain magnitude and a certain phase angle relative to the input. The harmonic excitations $\mathbf{f}(t)$ and the harmonic responses of the end-effector $\mathbf{q}(t)$ can be expressed as:

$$
\begin{aligned}
\mathbf{f}(t) & =\overline{\mathbf{f}} e^{j \omega t}, \\
\mathbf{q}(t) & =\overline{\mathbf{q}} e^{j \omega t},
\end{aligned}
$$

where $\overline{\mathbf{f}}$ and $\overline{\mathbf{q}}$ are the column vectors whose elements are complex numbers representing the amplitudes and the initial phases of the excitations and responses. Substituting eqs. (3.22) and (3.23) into eq. (3.21) yields:

$$
-{ }^{e} \mathbf{M} \omega^{2} \overline{\mathbf{q}} e^{j \omega t}+{ }^{e} \mathbf{K}_{E}(\omega) \overline{\mathbf{q}} e^{j \omega t}=\overline{\mathbf{f}} e^{j \omega t} .
$$

The Frequency Response Function (FRF) matrix can then be expressed as:

$$
\mathbf{H}(\omega)=\frac{\mathbf{q}(t)}{\mathbf{f}(t)}=\frac{\overline{\mathbf{q}}}{\overline{\mathbf{f}}}=\frac{1}{-\omega^{2}{ }^{e} \mathbf{M}+{ }^{e} \mathbf{K}_{E}(\omega)} .
$$

In the above equations, the system responses $\mathbf{q}(t)$ are displacement responses and $\mathbf{H}(\omega)$ is also called the dynamic compliance. Generally, velocity responses and acceleration responses can be also used in the vibration analysis. The velocity responses $\mathbf{v}(t)$ and the acceleration responses $\mathbf{a}(t)$ can be expressed as:

$$
\begin{aligned}
& \mathbf{v}(t)=\dot{\mathbf{q}}(t)=j \omega \overline{\mathbf{q}} e^{j \omega t}=\overline{\mathbf{v}} e^{j \omega t}, \\
& \mathbf{a}(t)=\ddot{\mathbf{q}}(t)=-\omega^{2} \overline{\mathbf{q}} e^{j \omega t}=\overline{\mathbf{a}} e^{j \omega t},
\end{aligned}
$$

where $\overline{\mathbf{v}}$ and $\overline{\mathbf{a}}$ are the column vectors whose elements are complex numbers representing the amplitudes and the initial phases of the velocity and acceleration responses: $\overline{\mathbf{v}}=j \omega \overline{\mathbf{q}}$ and $\overline{\mathbf{a}}=-\omega^{2} \overline{\mathbf{q}}$. By substituting eqs. (3.22), (3.23), (3.26) and (3.27) into eq. (3.21), the dynamic response functions of the end-effector under a harmonic excitation can be expressed as:

$$
\mathbf{H}_{V}(\omega)=\frac{\mathbf{v}(t)}{\mathbf{f}(t)}=\frac{\overline{\mathbf{v}}}{\overline{\mathbf{f}}}=\frac{j \omega}{-\omega^{2} e \mathbf{M}+{ }^{e} \mathbf{K}_{E}(\omega)},
$$




$$
\mathbf{H}_{A}(\omega)=\frac{\mathbf{a}(t)}{\mathbf{f}(t)}=\frac{\overline{\mathbf{a}}}{\overline{\mathbf{f}}}=\frac{-\omega^{2}}{-\omega^{2}{ }^{e} \mathbf{M}+{ }^{e} \mathbf{K}_{E}(\omega)},
$$

where $\mathbf{H}_{V}(\omega)$ and $\mathbf{H}_{A}(\omega)$ are also called the mobility and the impedance respectively. All the dynamic information of CDPRs are contained in these dynamic response functions, including the cable vibrations, the end-effector vibrations and their coupling. In addition, the dynamic responses of the end-effector can be plotted as functions of the frequency $\omega$. From these plots, the natural frequencies of CDPRs can be identified and the effect of cable dynamics on the system vibrations can be analyzed. This issue will be further detailed in the following example.

\subsection{A numerical Example}

In this section, a 6-DOF suspended CDPR driven by 8 cables used for the pick-and-place application is chosen as an example in order to illustrate the static and dynamic stiffness modeling of CDPRs through the proposed methods. Firstly, the configuration of the studied CDPR is described. Then, the static positioning error of the end-effector is calculated and the variation of the pose error with external load is presented to evaluate the static stiffness of the CDPR. After that, dynamic response functions are computed and the dynamic responses of the end-effector under a harmonic excitation are plotted as functions of the frequency $\omega$. The coupling of the cable dynamics and end-effector vibrations are shown in these plots. The effect of cable dynamics on the system dynamics can be also analyzed through these plots.

\subsubsection{Description of the studied CDPR}

The schematic diagram of the studied CDPR is represented in fig. 3.3. As shown, it is a 6-DOF suspended CDPR driven by 8 cables. There are 8 attachment points $B_{i}(i=1,2, \cdots, 8)$ on the 4 vertical poles, and 8 corresponding attachment points $A_{i}$ on the end-effector. These attachment points are connected by 8 driving cables, respectively. The end-effector can change its pose through controlling the length and tension of the 8 cables. The configuration parameters are given in table 3.1, which is close to the parameters of the CoGiRo [Lamaury 2013]. A set of anti-rust steel cables are used in the simulations, and the cable parameters are the same with those in section 2.3 (table 2.1). 


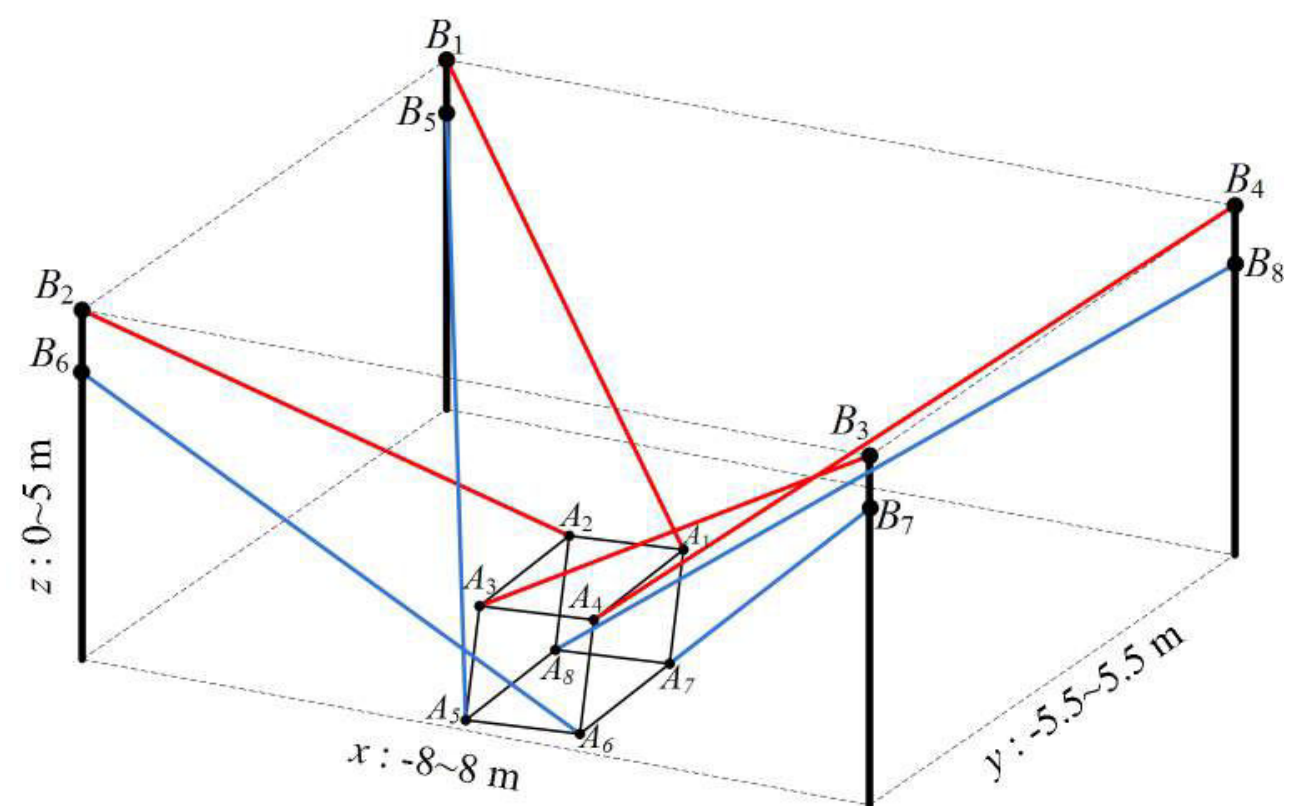

Fig. 3.3 The schematic diagram of the studied CDPR (6-DOF suspended by 8 cables)

Table 3.1 Configuration parameters of the studied CDPR: coordinates of $B_{i}$ expressed in global frame $\mathfrak{R}_{G}$; coordinates of $A_{i}$ expressed in end-effector frame $\mathfrak{R}_{e}$

\begin{tabular}{|l|c|c|c||c|c|c|c|}
\hline & $x(\mathrm{~m})$ & $y(\mathrm{~m})$ & $z(\mathrm{~m})$ & & $x(\mathrm{~m})$ & $y(\mathrm{~m})$ & $z(\mathrm{~m})$ \\
\hline \hline$A_{1}$ & 0.48 & 0.32 & 0.32 & $B_{1}$ & -8 & 5.5 & 5 \\
\hline$A_{2}$ & -0.48 & 0.32 & 0.32 & $B_{2}$ & -8 & -5.5 & 5 \\
\hline$A_{3}$ & -0.48 & -0.32 & 0.32 & $B_{3}$ & 8 & -5.5 & 5 \\
\hline$A_{4}$ & 0.48 & -0.32 & 0.32 & $B_{4}$ & 8 & 5.5 & 5 \\
\hline$A_{5}$ & -0.48 & -0.32 & -0.32 & $B_{5}$ & -8 & 5.5 & 4 \\
\hline$A_{6}$ & 0.48 & -0.32 & -0.32 & $B_{6}$ & -8 & -5.5 & 4 \\
\hline$A_{7}$ & 0.48 & 0.32 & -0.32 & $B_{7}$ & 8 & -5.5 & 4 \\
\hline$A_{8}$ & -0.48 & 0.32 & -0.32 & $B_{8}$ & 8 & 5.5 & 4 \\
\hline
\end{tabular}

\subsubsection{Static stiffness modeling of the studied CDPR}

For pick-and-place applications, the static stiffness along the vertical direction is usually low due to the cable sag and elasticity, especially when the end-effector is unloaded. Meanwhile, the static positioning accuracy along the vertical direction is quite important to accurately pick up and lay down cargoes. Therefore, the stiffness and positioning accuracy along the vertical direction are mainly focused to ensure the accuracy of pick-and-place applications.

According to the definition of static pose error in section 3.2.2, the static pose errors of the end-effector along the vertical direction (axis- $z_{G}$ ) over a sub-workspace are computed and represented in figs. 3.4 to 3.7. The sub-workspace is defined as $-3 \mathrm{~m} \leqslant x_{G} \leqslant 3 \mathrm{~m}$, 


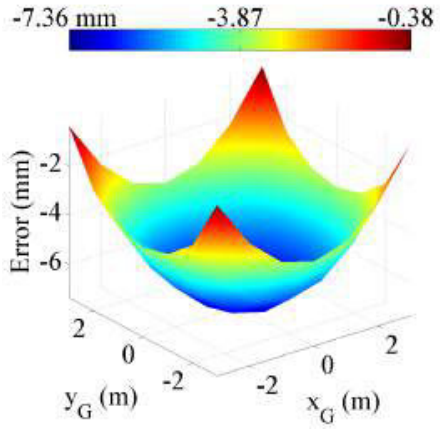

(a) Global pose error

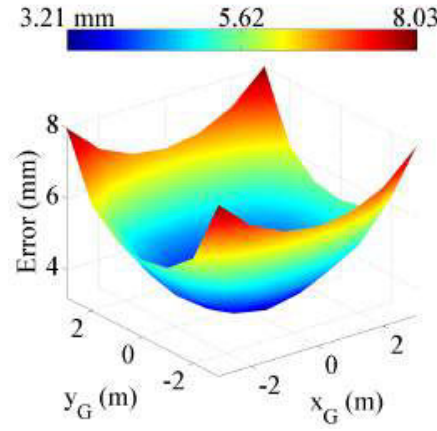

(b) Sag-introduced pose error

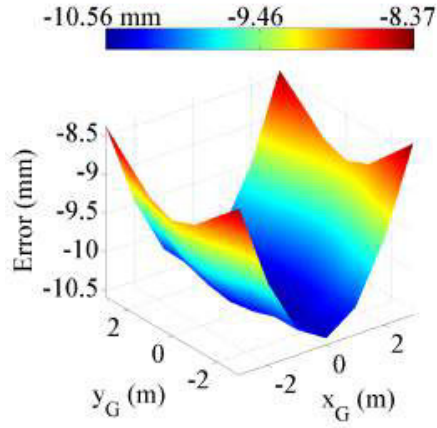

(c) Axial-elasticity-introduced pose error

Fig. 3.4 Static pose error of the end-effector along axis- $z_{G}$ over a sub-workspace $(-3 \mathrm{~m} \leqslant$ $x_{G} \leqslant 3 \mathrm{~m},-3 \mathrm{~m} \leqslant y_{G} \leqslant 3 \mathrm{~m}, z_{G}=0.5 \mathrm{~m}$ ) for $\phi 4 \mathrm{~mm}$ cable and $30 \mathrm{~kg}$ external load

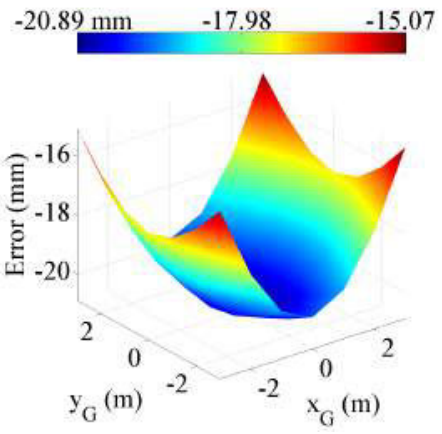

(a) Global pose error

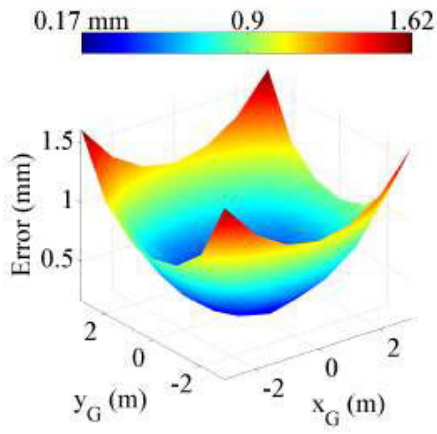

(b) Sag-introduced pose error

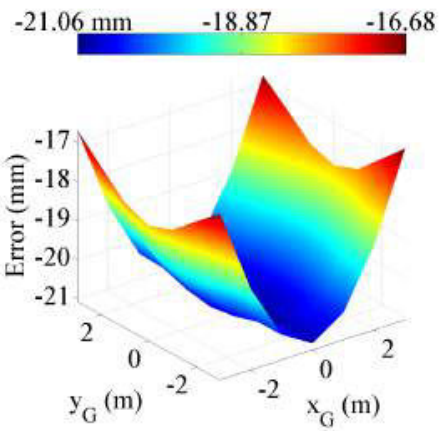

(c) Axial-elasticity-introduced pose error

Fig. 3.5 Static pose error of the end-effector along axis- $z_{G}$ over a sub-workspace $(-3 \mathrm{~m} \leqslant$ $\left.x_{G} \leqslant 3 \mathrm{~m},-3 \mathrm{~m} \leqslant y_{G} \leqslant 3 \mathrm{~m}, z_{G}=0.5 \mathrm{~m}\right)$ for $\phi 4 \mathrm{~mm}$ cable and $60 \mathrm{~kg}$ external load

Table 3.2 Average values of the pose errors in the sub-workspace $\left(-3 \mathrm{~m} \leqslant x_{G} \leqslant 3 \mathrm{~m}\right.$, $\left.-3 \mathrm{~m} \leqslant y_{G} \leqslant 3 \mathrm{~m}, z_{G}=0.5 \mathrm{~m}\right)$

\begin{tabular}{|c|c|c|c|c|}
\hline \multirow{2}{*}{$\begin{array}{l}\text { Cable } \\
\text { diameter }\end{array}$} & \multirow{2}{*}{$\begin{array}{l}\text { External } \\
\text { load }\end{array}$} & \multicolumn{3}{|c|}{ Average pose error } \\
\cline { 2 - 5 }$\phi 4 \mathrm{~mm}$ & $30 \mathrm{~kg}$ & $5.4 \mathrm{~mm}$ & $-9.7 \mathrm{~mm}$ & $-4.3 \mathrm{~mm}$ \\
\cline { 2 - 5 } & $60 \mathrm{~kg}$ & $0.8 \mathrm{~mm}$ & $-19.3 \mathrm{~mm}$ & $-18.5 \mathrm{~mm}$ \\
\hline \multirow{2}{*}{$\phi 8 \mathrm{~mm}$} & $30 \mathrm{~kg}$ & $55.0 \mathrm{~mm}$ & $-2.4 \mathrm{~mm}$ & $52.6 \mathrm{~mm}$ \\
\cline { 2 - 5 } & $60 \mathrm{~kg}$ & $18.1 \mathrm{~mm}$ & $-4.9 \mathrm{~mm}$ & $13.2 \mathrm{~mm}$ \\
\hline
\end{tabular}

$-3 \mathrm{~m} \leqslant y_{G} \leqslant 3 \mathrm{~m}, z_{G}=0.5 \mathrm{~m}$, where the rotational angles around axis- $x_{G}, y_{G}$, and $z_{G}$ are all zeros. The global pose errors represented in figs. 3.4a, 3.5a, 3.6a and 3.7a are computed by the sagging cable model, where both cable sag and elasticity are considered. The axial- 


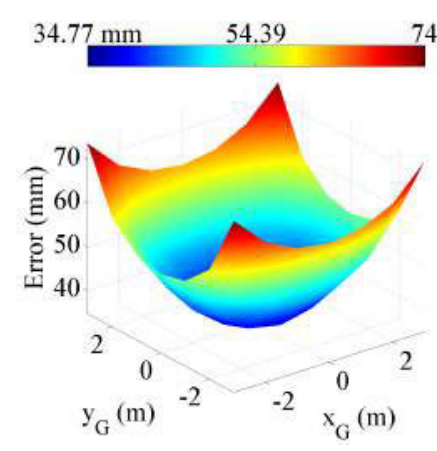

(a) Global pose error

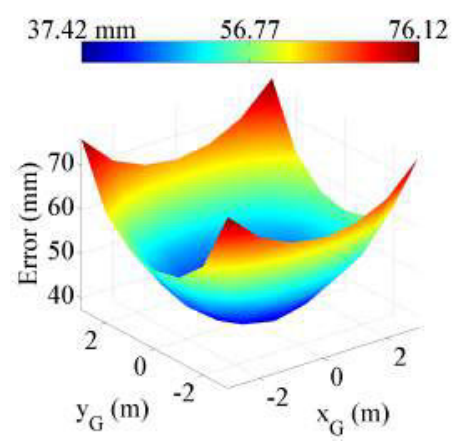

(b) Sag-introduced pose error

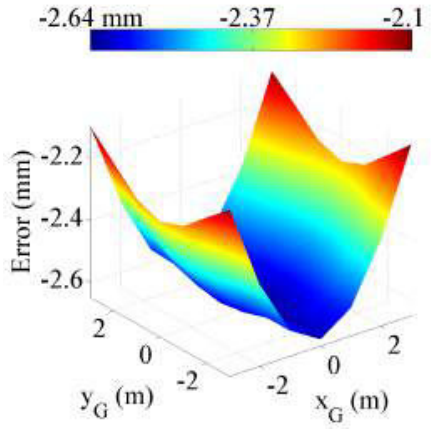

(c) Axial-elasticity-introduced pose error

Fig. 3.6 Static pose error of the end-effector along axis- $z_{G}$ over a sub-workspace $(-3 \mathrm{~m} \leqslant$ $\left.x_{G} \leqslant 3 \mathrm{~m},-3 \mathrm{~m} \leqslant y_{G} \leqslant 3 \mathrm{~m}, z_{G}=0.5 \mathrm{~m}\right)$ for $\phi 8 \mathrm{~mm}$ cable and $30 \mathrm{~kg}$ external load

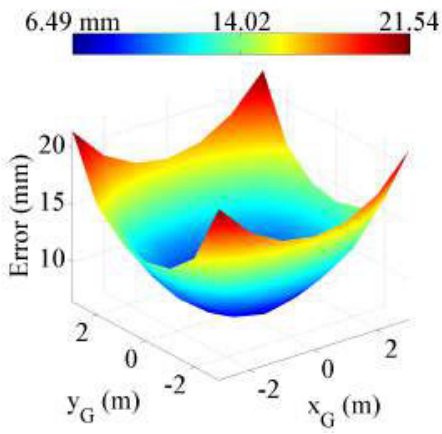

(a) Global pose error

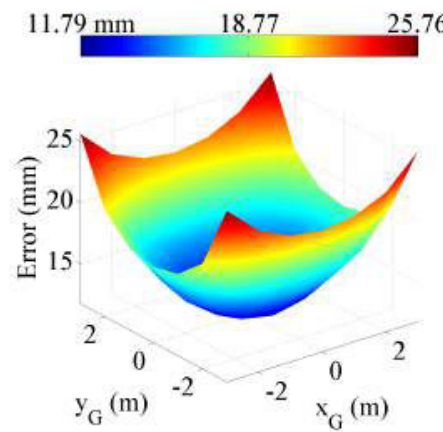

(b) Sag-introduced pose error

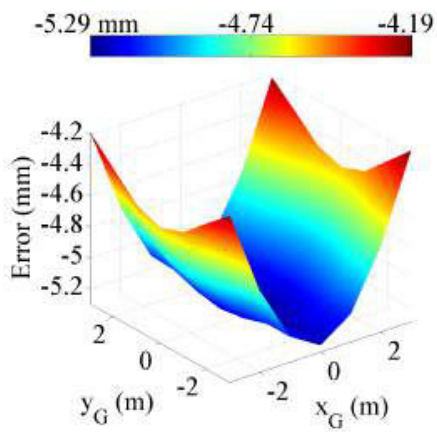

(c) Axial-elasticity-introduced pose error

Fig. 3.7 Static pose error of the end-effector along axis- $z_{G}$ over a sub-workspace $(-3 \mathrm{~m} \leqslant$ $\left.x_{G} \leqslant 3 \mathrm{~m},-3 \mathrm{~m} \leqslant y_{G} \leqslant 3 \mathrm{~m}, z_{G}=0.5 \mathrm{~m}\right)$ for $\phi 8 \mathrm{~mm}$ cable and $60 \mathrm{~kg}$ external load

elasticity-introduced pose errors represented in figs. 3.4c, 3.5c, 3.6c and 3.7c are computed by the spring cable model, where only axial cable elasticity is considered. Due to the elongation of cables, the position of the end-effector calculated by the spring cable model is lower than that calculated by the ideal cable model. Therefore, according to the definition of pose error in section 3.2.2, the axial-elasticity-introduced pose errors are all negative. The sag-introduced pose errors represented in figs. $3.4 \mathrm{~b}, 3.5 \mathrm{~b}, 3.6 \mathrm{~b}$ and $3.7 \mathrm{~b}$ are the difference between the global pose errors and the axial-elasticity-introduced pose errors, where only cable sag is considered. As is known, the chord length of a sagging curve is shorter than the arc length of the sagging curve. Thus, the position of the end-effector due to cable sag is higher than the position calculated by the ideal cable model. Therefore, the sag-introduced 
pose errors are all positive. Through these figures, the effect of cable sag and/or axial cable elasticity on the robot's pose error can be clearly, and the average values of the pose errors in the sub-workspace are computed and listed in table 3.2.

As shown in figs. 3.4 to 3.7, the pose error of the end-effector depends on the pose of the end-effector. In general, the pose error is smallest near the center of the sub-workspace, while it becomes bigger near the edge of the workspace. Moreover, the pose error of the end-effector is also associated with the cable parameters and the external loads.

As explained before, the pose error of the end-effector has two sources: the sag-introduced pose error and the axial-elasticity-introduced pose error. From these figures, it is indicated that the major source of the pose error is not the same for different cable diameters and external loads. Firstly, for the CDPR with $\phi 4 \mathrm{~mm}$ cable and $60 \mathrm{~kg}$ external load (fig. 3.5), the axial-elasticity-introduced pose error has a prime contribution to the global pose error, while sag-introduced pose error is small enough to be neglected. On the contrary, for the CDPR with $\phi 8 \mathrm{~mm}$ cable and $30 \mathrm{~kg}$ external load (fig. 3.6), the sag-introduced pose error plays a leading role while the axial-elasticity-introduced pose error is negligible. In addition, for the CDPR with $\phi 4 \mathrm{~mm}$ cable and $30 \mathrm{~kg}$ external load and the CDPR with $\phi 8 \mathrm{~mm}$ cable and 60 $\mathrm{kg}$ external load, both sag-introduced pose error and axial-elasticity-introduced pose error are non-negligible. The global pose error is a mix of these two sources.

To sum up, the sag-introduced pose error is the major source of global pose error for CDPRs with heavier cable and lighter external load. While the axial-elasticity-introduced pose error becomes the major source of global pose error for CDPRs with lighter cable and heavier external load. These simulations are quite useful for the design and operation of CDPRs. This issue will be further discussed in Chapter 4.

\subsubsection{Dynamic stiffness modeling of the studied CDPR}

In this section, the dynamic stiffness and vibration of the studied CDPR are analyzed through the natural frequency identification of the robot. Firstly, the FRFs are calculated and plotted for certain poses of the end-effector in the workspace. From these plots, the robot 's natural frequencies can be identified. Then the first natural frequency of the end-effector and the 8 driving cables are identified and plotted for each pose of the end-effector in the subworkspace. In the following simulations, the $\phi 4 \mathrm{~mm}$ cables are used as the driving cables, and the cable parameters can be found in section 3.4.1. The total mass of the end-effector and the external load is $60 \mathrm{~kg}$, and their rotational inertia around axis- $x_{e}, y_{e}$ and $z_{e}$ are $7.1,11.3$ and $11.3 \mathrm{~kg} \cdot \mathrm{m}^{2}$, respectively. In this example, axis- $x_{e}, y_{e}$ and $z_{e}$ coincide with the principal 
axes of the end-effector.

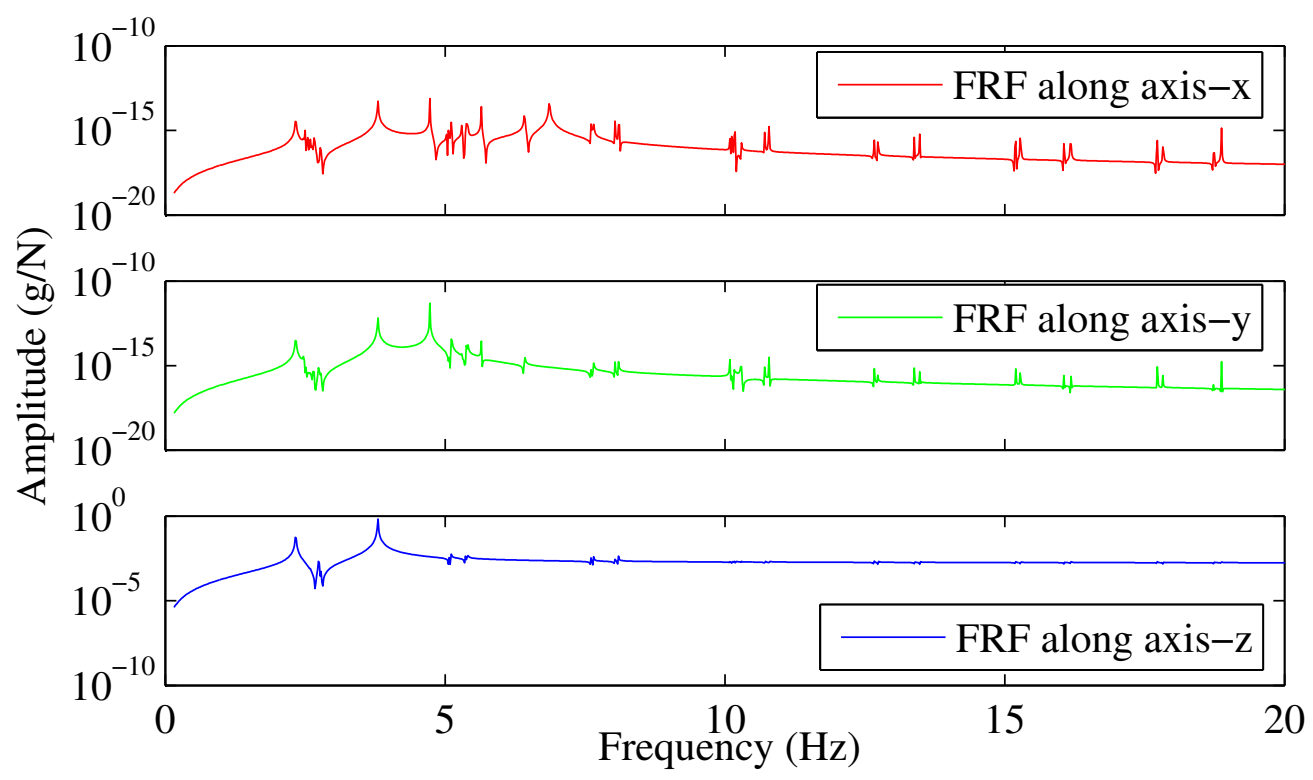

(a) FRFs between the acceleration response of the end-effector along axis- $x_{e}, y_{e}, z_{e}$ and the excitation force along axis- $z_{e}$

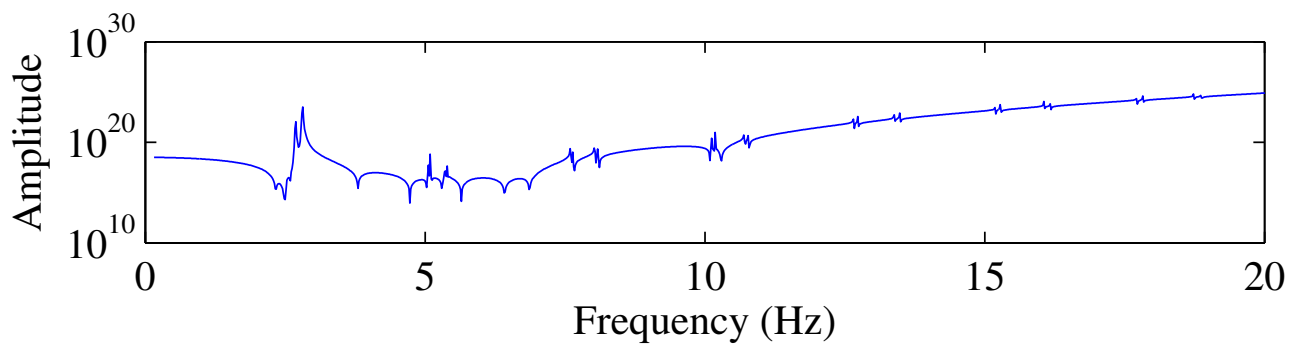

(b) Amplitude variation of the determinant of $\omega^{2} \mathbf{E}-\operatorname{inv}\left({ }^{e} \mathbf{M}\right)^{e} \mathbf{K}_{E}(\omega)$

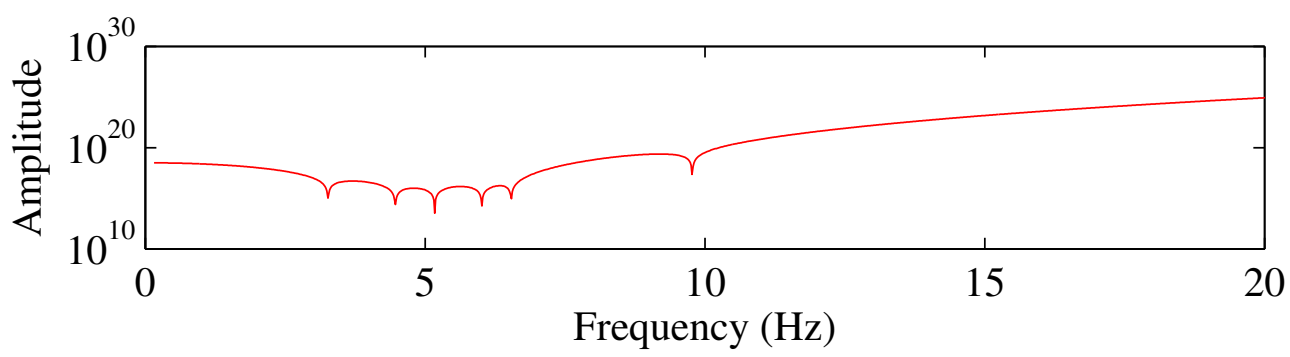

(c) Amplitude variation of the determinant of $\omega^{2} \mathbf{E}-\operatorname{inv}\left({ }^{e} \mathbf{M}\right) \mathbf{K}_{\text {static }}$

Fig. 3.8 Vibration analysis of the studied CDPR when the end-effector is at the center of the sub-workspace $\left(x_{G}=0 \mathrm{~m}, y_{G}=0 \mathrm{~m}\right.$ and $\left.z_{G}=0.5 \mathrm{~m}\right)$

Figure 3.8 shows the vibration analyses of the studied CDPR when the end-effector is at the center of the sub-workspace where $x_{G}=0 \mathrm{~m}, y_{G}=0 \mathrm{~m}$ and $z_{G}=0.5 \mathrm{~m}$ and the rotational 
angles around axis- $x_{G}, y_{G}$, and $z_{G}$ are all zeros. The horizontal axis represents frequency whose unit is $\mathrm{Hz}$, and the vertical axis in fig. 3.8a represents the amplitude of the acceleration response of the end-effector whose unit is the gravitational acceleration $g$. The limits of cables parameters defined by eqs. (2.40) and (2.41) in Chapter 2 are verified for each driving cable to make sure the DSM method is applicable.

According to the dynamic stiffness model proposed in section 3.3 and eq. (3.29), the FRFs between the acceleration response of the end-effector along axis- $x_{e}, y_{e}, z_{e}$ and the excitation force along axis- $z_{e}$ are calculated and plotted in fig. 3.8a. The peaks in these FRF plots correspond to the natural frequencies of the global system, considering the modes of the cables and the rigid-body modes of the end-effector suspended on the stiffness of the cables. From these resonances, the natural frequencies of the studied CDPR can be identified. For example, the fundamental frequency of the studied CDPR is $2.4 \mathrm{~Hz}$. The robot dynamic described by the FRFs can be also studied by the determinant of $\omega^{2} \mathbf{E}-\operatorname{inv}\left({ }^{e} \mathbf{M}\right)^{e} \mathbf{K}_{E}(\omega)$ because it contains all the dynamic information of the robot. From the determinant plots fig. 3.8b, the natural frequencies of the studied CDPR can be identified and the results are the same with those obtained by the FRF plots.

As a contrast, the simulation result obtained by the static stiffness matrix $\mathbf{K}_{\text {static }}$ is presented in fig. 3.8c, where cable dynamics is totally neglected in $\mathbf{K}_{\text {static }}$. Thus the peaks in fig. 3.8c only correspond to the rigid-body modes of the end-effector suspended on the static stiffness of the cables (considering only elasticity and sag). From the comparison of fig. $3.8 \mathrm{~b}$ and fig. 3.8c, it is indicated that cable dynamics can affect the dynamic behaviors of CDPRs by adding new resonances and changing the value of natural frequencies. These simulations are useful for the dynamic stiffness analysis of CDPRs, from which cable vibration, endeffector vibration and their coupling can be represented. The dynamic stiffness analysis will be further detailed in Chapter 4.

As explained, the dynamic stiffness of CDPRs depends on the pose of the end-effector. Therefore, it is necessary to analyze the dynamic stiffness in the workspace of CDPRs. As known, the first natural frequency is usually the most important for the vibration analysis of CDPRs. Figure 3.9 shows the first natural frequency of the studied CDPR over the subworkspace defined as $-3 \mathrm{~m} \leqslant x_{G} \leqslant 3 \mathrm{~m},-3 \mathrm{~m} \leqslant y_{G} \leqslant 3 \mathrm{~m}, z_{G}=0.5 \mathrm{~m}$, where the rotational angles around axis- $x_{G}, y_{G}$, and $z_{G}$ are all zeros. It should be noted that the limits of cables parameters by eqs. (2.40) and (2.41) are verified for each driving cable at each pose of the end-effector in the studied sub-workspace. Figure 3.9a represents the first natural frequency of the end-effector over the sub-workspace. As shown, the natural frequency is the highest when the end-effector is at the center of the workspace and becomes smaller and smaller 


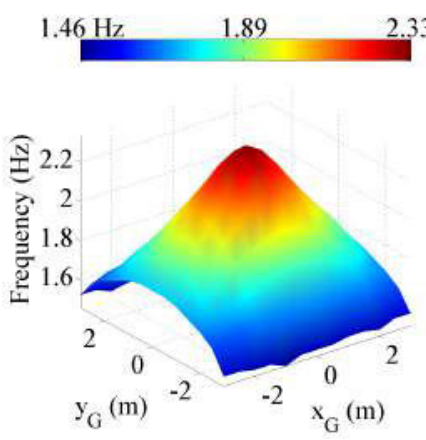

(a) The end-effector

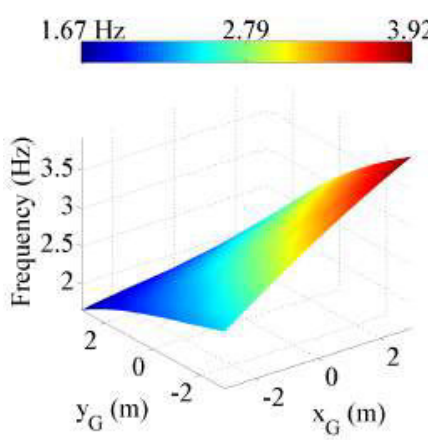

(d) The $3^{\text {rd }}$ cable

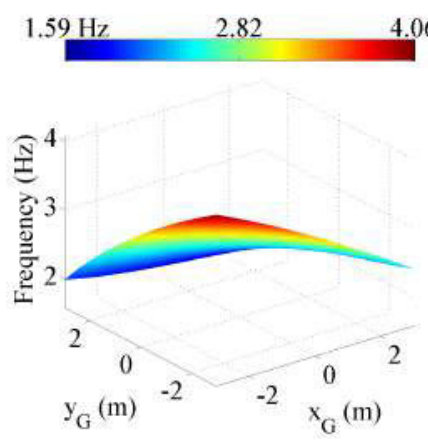

(g) The $6^{\text {th }}$ cable

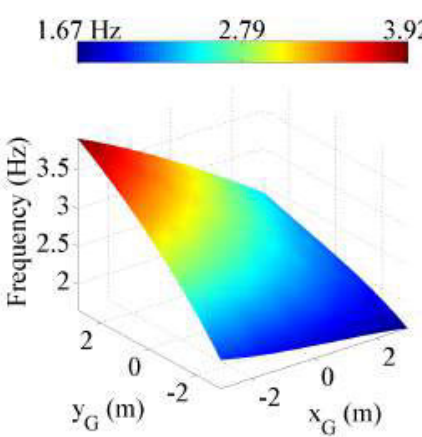

(b) The $1^{\text {st }}$ cable

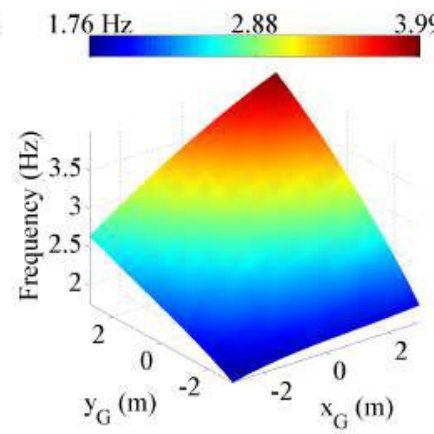

(e) The $4^{\text {th }}$ cable

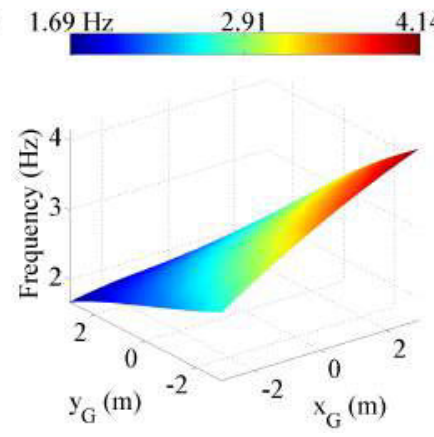

(h) The $7^{\text {th }}$ cable

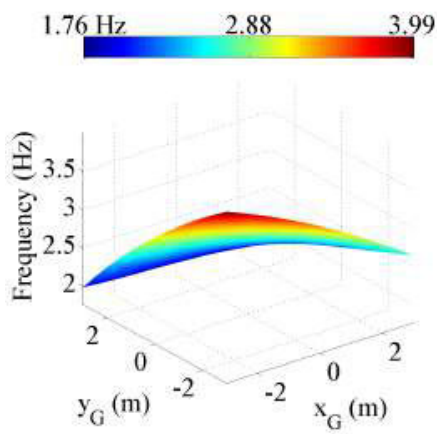

(c) The $2^{\text {nd }}$ cable

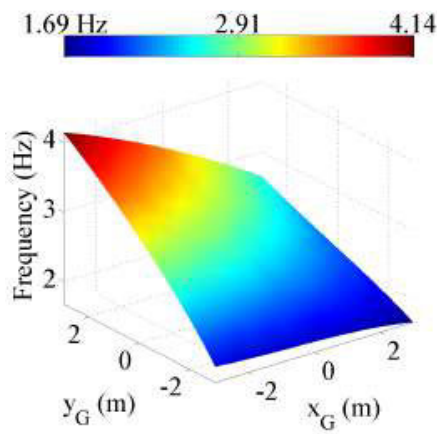

(f) The $5^{\text {th }}$ cable

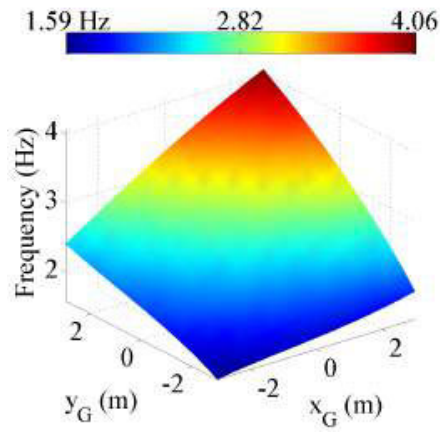

(i) The $8^{\text {th }}$ cable

Fig. 3.9 The first natural frequency of the studied CDPR over a sub-workspace $(-3 \mathrm{~m} \leqslant$ $\left.x_{G} \leqslant 3 \mathrm{~m},-3 \mathrm{~m} \leqslant y_{G} \leqslant 3 \mathrm{~m}, z_{G}=0.5 \mathrm{~m}\right)$ 
when the end-effector moves to the edge of the workspace, which means that the dynamic stiffness of the CDPR at the central pose is better than that at the edge poses. In addition, the first natural frequencies of the 8 driving cables over the sub-workspace are plotted in figs. $3.9 \mathrm{~b}$ to $3.9 \mathrm{i}$. to illustrate the variations of the cable dynamics over the workspace.

\subsection{Summary of the chapter}

This chapter presents the static and dynamic stiffness modeling of CDPRs. For static part, both the cable elasticity and the effect of cable weight on the static cable profile are considered. The pose error of the end-effector is defined and computed by the direct kinematic model of CDPRs. The static stiffness is evaluated by the variation of the pose error with external load. The main purpose of static stiffness modeling is to analyze and improve the static positioning accuracy of CDPRs, especially for the pick-and-place application.

For the dynamic part, a new dynamic stiffness model of CDPRs is proposed. This dynamic model has a complete description of the dynamic behaviors of CDPRs, which considers the cable dynamics, the end-effector dynamics and their coupling. As far as we know, it is the first time that the DSM method is applied in the modeling of CDPRs. The oscillating model of the end-effector around a static equilibrium is established through Lagrange's equations, and dynamic response functions are calculated under a harmonic excitation. The dynamic stiffness modeling aims to study the effect of cable dynamic on the dynamic behaviors of CDPRs and further reduce the vibration of the system.

At the end of the chapter, an example is given to illustrate how to use the proposed methods in the stiffness modeling of CDPRs. The simulation results show that cable dynamic has a significant influence to the system vibration. Further analysis of the static and dynamic behaviors of CDPRs will be detailed in Chapter 4 . 


\section{Chapter 4}

\section{Experimental Validation and Stiffness Analysis}

\section{Contents}

4.1 Description of the CDPR prototypes . . . . . . . . . . . 58

4.2 Static experiments and static stiffness analysis $\ldots \ldots \ldots \ldots$. . . . 61

4.3 Modal experiments and dynamic stiffness analysis . . . . . . . . 64

4.4 Dynamic trajectory experiments and dynamic stiffness analysis . . . 75

4.5 Summary of the chapter . . . . . . . . . . . . 83

In the previous chapters, the static and dynamic cable models are introduced, based on which the static and dynamic models of CDPRs are proposed. This chapter will focus on the experimental validation of the proposed models. Three kinds of experiments are carried out:

- Static experiment:

The purpose of the static experiment is to validate the static sagging cable model introduced in Chapter 2 and confirm the recent results obtained by [Nguyen+2013] in 2013, and to analyze the static stiffness of CDPRs through the new performance index: the variation of the pose error with the external load.

- Dynamic modal experiment:

The purpose of the dynamic modal experiment is to validate the introduced dynamic cable model and the proposed dynamic stiffness model of CDPRs, also analyze the coupling between the cable vibration and the end-effector vibration. 
- Dynamic experiment along a trajectory:

The purpose of the dynamic trajectory experiment is to study the robot dynamics along a trajectory and analyze the cable dynamics, end-effector dynamics and their coupling.

In order to achieve the above purposes, two CDPR prototypes are used in the experiments.

- A 6-DOF CDPR prototype suspended by 6 cables:

The 6-cable prototype is simplified without actuators. Two groups of cables with fixed cable lengths are used to achieve two different poses of the end-effector. In addition, the total mass of the end-effector can be changed in a large range by adding or moving the dead weight. Static experiment is carried out to validate the introduced sagging cable model and the static stiffness model of CDPRs.

- A CDPR prototype with 6-DOF driven by 8 cables, the CoGiRo:

The CoGiRo [Lamaury 2013] is a complete CDPR mainly developed by LIRMM ${ }^{1}$ and TECNALIA ${ }^{2}$. Dynamic modal experiments are made at certain poses of the endeffector and dynamic trajectory experiments are carried out, including free vibration experiments after an emergency stop during a trajectory, and dynamic analysis of CDPRs along an entire trajectory.

This chapter is organized as following: firstly, the two CDPR prototypes are described. Then static experiment, the dynamic modal experiment and the dynamic trajectory experiment together with the static and dynamic stiffness analysis of CDPRs are presented.

\subsection{Description of the CDPR prototypes}

\subsubsection{The 6-cable CDPR prototype}

Figure 4.1 presents the 6-DOF CDPR suspended by 6 cables. As shown in fig. 4.1, there are 6 attachment points $\left(B_{i}, i=1,2 \cdots 6\right)$ on the three vertical poles and 6 attachment points $\left(A_{i}, i=1,2 \cdots 6\right)$ on the end-effector. The corresponding attachment points $A_{i}$ and $B_{i}$ are connected by 6 cables. The configuration of this prototype is similar to the CDPR presented in [Gouttefarde+ 2012]. The dimensions of this prototype are about $10 \mathrm{~m}$ long, $6 \mathrm{~m}$ wide and $5 \mathrm{~m}$ high. Detailed parameters of this prototype are given in table 4.1.

Since the main objective of this prototype is to validate the proposed static and dynamic stiffness models of CDPRs at certain poses of the end-effector in the workspace, the prototype

\footnotetext{
${ }^{1}$ LIRMM: le Laboratoire d'Informatique, de Robotique et de Microélectronique de Montpellier

${ }^{2}$ TECNALIA is a company: http://www.tecnalia.com/en/
} 


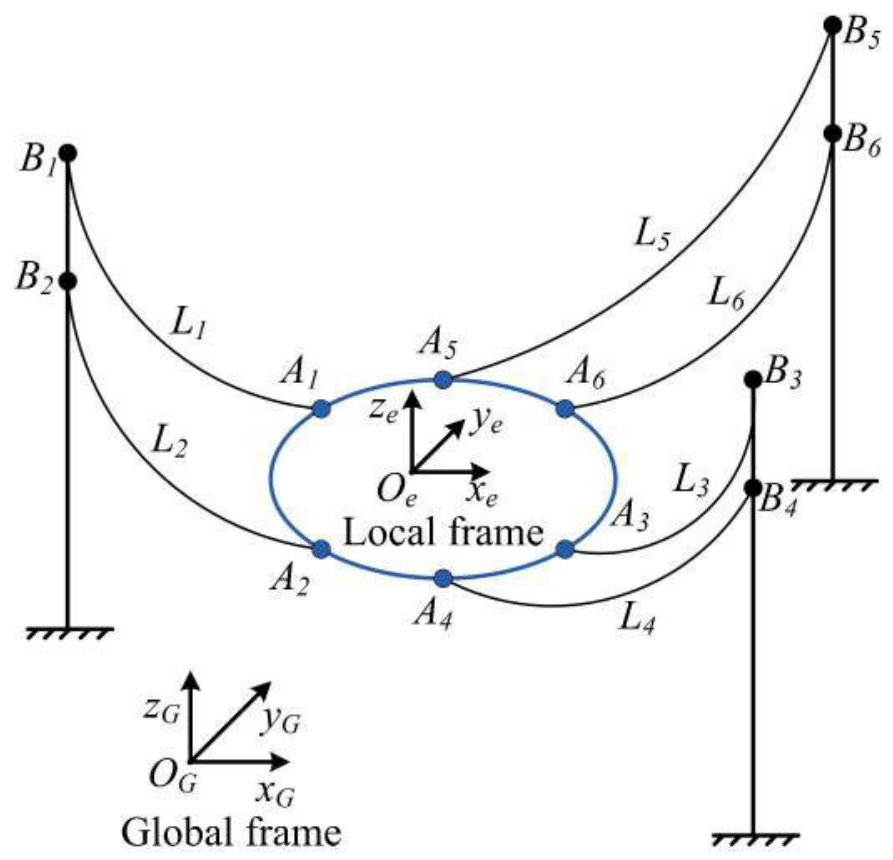

Fig. 4.1 The CDPR prototype with 6 cables

Table 4.1 Configuration parameters of the 6-cable CDPR prototype: coordinates of the points $B_{i}$ in the global frame $\mathfrak{R}_{G}$ and that of $A_{i}$ in the local frame $\mathfrak{R}_{e}$

\begin{tabular}{|c|c|c|c|l|l|c|c|c|}
\hline$(\mathrm{m})$ & $x$ & $y$ & $z$ & & $x$ & $y$ & $z$ \\
\hline \hline$A_{1}$ & -0.025 & -0.143 & 0 & $B_{1}$ & 5.327 & -2.267 & 4.193 \\
\hline$A_{2}$ & 0.136 & -0.050 & 0 & $B_{2}$ & 5.327 & -2.267 & 3.822 \\
\hline$A_{3}$ & 0.136 & 0.050 & 0 & $B_{3}$ & 5.327 & 2.267 & 4.193 \\
\hline$A_{4}$ & -0.025 & 0.143 & 0 & $B_{4}$ & 5.327 & 2.267 & 3.822 \\
\hline$A_{5}$ & -0.111 & 0.093 & 0 & $B_{5}$ & -5.775 & 0.010 & 4.193 \\
\hline$A_{6}$ & -0.111 & -0.093 & 0 & $B_{6}$ & -5.775 & 0.010 & 3.822 \\
\hline
\end{tabular}

Table 4.2 Cable parameters of the 6-cable CDPR prototype

\begin{tabular}{|c|c|c|c|c|c|c|c|}
\hline \multicolumn{2}{|c|}{ Diameter } & \multicolumn{3}{|c|}{$\phi 4 \mathrm{~mm}$} & \multicolumn{3}{|c|}{$\phi 8 \mathrm{~mm}$} \\
\hline \multirow{2}{*}{$\begin{array}{c}\text { Length } \\
(\mathrm{mm})\end{array}$} & $l_{u s 1} \sim l_{u s 3}$ & 6863 & 6565 & 6756 & 4859 & 4552 & 4830 \\
\hline & $l_{u s 4} \sim l_{u s 6}$ & 6663 & 6801 & 6604 & 4586 & 9417 & 9284 \\
\hline \multicolumn{2}{|c|}{ Young's Modulus } & \multicolumn{3}{|c|}{$20.0 \mathrm{GPa}$} & \multicolumn{3}{|c|}{$20.1 \mathrm{GPa}$} \\
\hline \multicolumn{2}{|c|}{ Mass per meter } & \multicolumn{3}{|c|}{$0.067 \mathrm{~kg} / \mathrm{m}$} & \multicolumn{3}{|c|}{$0.251 \mathrm{~kg} / \mathrm{m}$} \\
\hline
\end{tabular}


is built without motors and winches. The attachment points $B_{i}$ are fixed on the poles. Two sets of anti-rust steel cables are used. One set is $\phi 4 \mathrm{~mm}$ in diameter and the other set is $\phi 8$ $\mathrm{mm}$ in diameter. Two poses of the end-effector can be achieved with these two sets of cables. One pose is near the center of the workspace $(0,0,0.5 \mathrm{~m})$, the other is near the edge of the workspace $(3,0,0.5 \mathrm{~m})$. The rotational angles around axis- $x_{G}, y_{G}$ and $z_{G}$ are approximate to zeros. The Young's modulus of the driving cables is identified using a material testing machine. All cables work within their linear elastic region. The relevant cable parameters are given in table 4.2 .

\subsubsection{The 8-cable CDPR prototype}

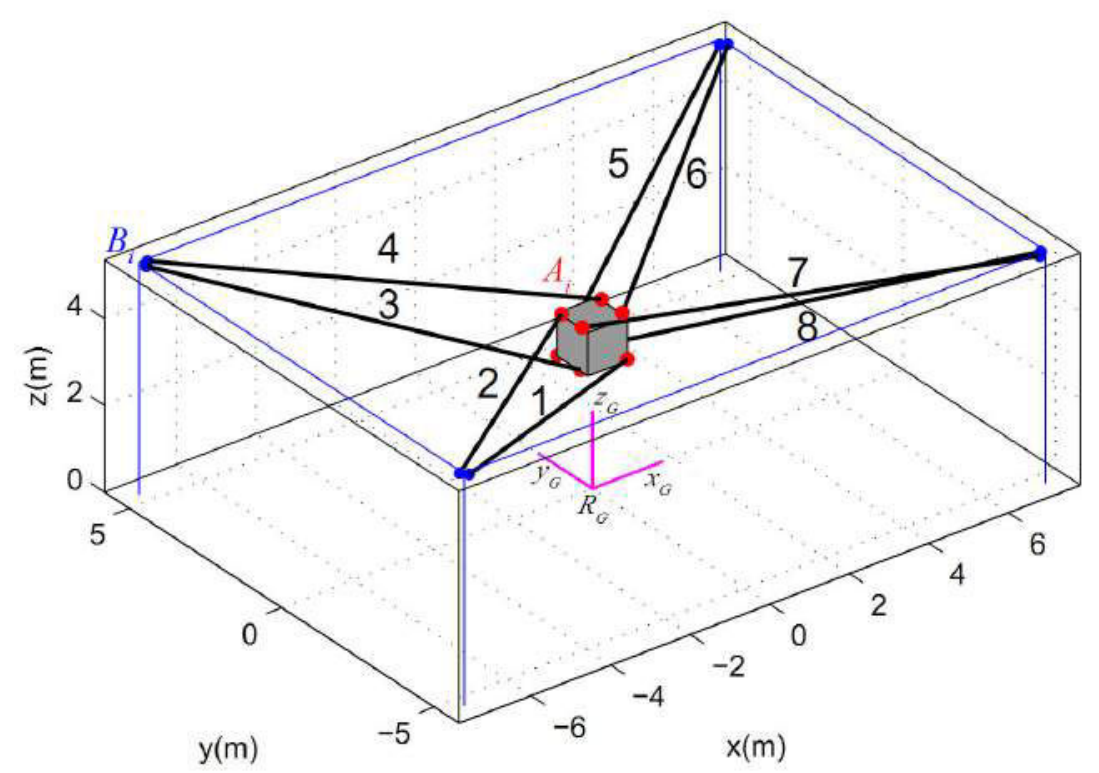

Fig. 4.2 The schematic diagram of the CoGiRo [Lamaury+ 2013]

The schematic diagram of the CoGiRo is represented in fig. 4.2. The configuration parameters of the CoGiRo is listed in table 4.3. This robot uses $\phi 4 \mathrm{~mm}$ anti-rust steel cables as driving cables. The Young's Modulus of the cable is $20 \mathrm{Gpa}$ and the linear weight of the cable is $0.067 \mathrm{~kg} / \mathrm{m}$. More details of this robot can be found in [Lamaury+ 2013]. 
Table 4.3 Configuration parameters of the CoGiRo: coordinates of $B_{i}$ expressed in global frame $\mathfrak{R}_{G}$; coordinates of $A_{i}$ expressed in end-effector frame $\mathfrak{R}_{e}$

\begin{tabular}{|c|c|c|c||c|c|c|c|}
\hline & $x(\mathrm{~m})$ & $y(\mathrm{~m})$ & $z(\mathrm{~m})$ & & $x(\mathrm{~m})$ & $y(\mathrm{~m})$ & $z(\mathrm{~m})$ \\
\hline \hline$B_{1}$ & -7.224 & -5.359 & 5.468 & $A_{1}$ & 0.500 & -0.507 & 0.555 \\
\hline$B_{2}$ & -7.435 & -5.058 & 5.477 & $A_{2}$ & -0.488 & 0.361 & 0.554 \\
\hline$B_{3}$ & -7.425 & 5.196 & 5.486 & $A_{3}$ & -0.500 & -0.260 & 0.555 \\
\hline$B_{4}$ & -7.210 & 5.497 & 5.495 & $A_{4}$ & 0.503 & 0.342 & 0.548 \\
\hline$B_{5}$ & 7.139 & 5.463 & 5.481 & $A_{5}$ & -0.500 & 0.507 & 0.555 \\
\hline$B_{6}$ & 7.440 & 5.158 & 5.494 & $A_{6}$ & 0.497 & -0.353 & 0.554 \\
\hline$B_{7}$ & 7.415 & -5.089 & 5.481 & $A_{7}$ & 0.499 & 0.260 & 0.549 \\
\hline$B_{8}$ & 7.113 & -5.388 & 5.492 & $A_{8}$ & -0.495 & -0.333 & 0.554 \\
\hline
\end{tabular}

\subsection{Static experiments and static stiffness analysis}

The objective of the static experiments is to validate the static sagging cable model and the proposed static stiffness model of CDPRs. In this section, the variation of the pose error of the end-effector with the external load is studied by both simulations and experiments on the 6-cable CDPR prototype to demonstrate the validity of the proposed models.

\subsubsection{Static experimental setup}

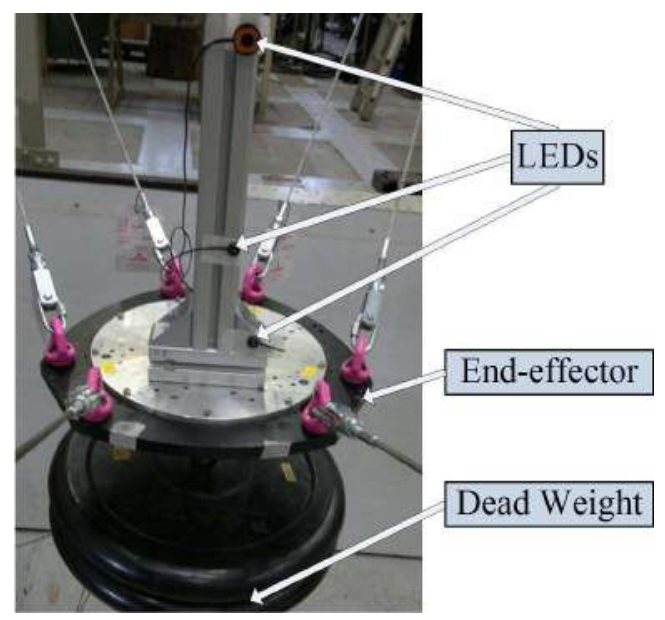

Fig. 4.3 Static experimental setup

As shown in fig. 4.3, the experimental setup consists of the 6-cable CDPR prototype, the precise multi-camera system Nikon Metrology K600-10 and a loading device connected to the center of the end-effector. Technical details of the experimental equipments can be found in the Appendix. The LEDs of the multi-camera system are attached to the end-effector and 
the pose of the end-effector (both position and orientation) can be measured and recorded by the system. The mass of the end-effector can be adjusted from 10 to $86 \mathrm{~kg}$ by adding dead weight to the loading device.

\subsubsection{Static experimental results and analysis}

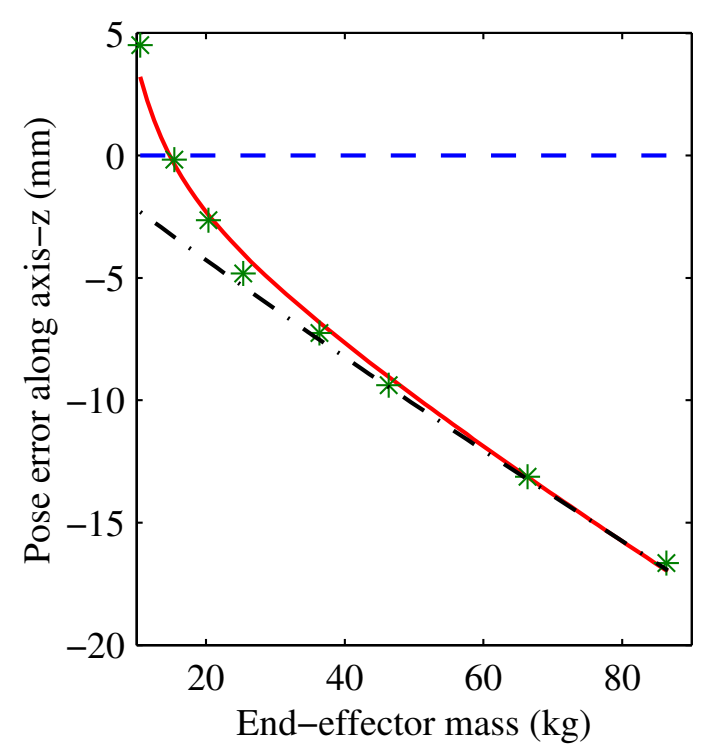

(a) Cable diameter: $\phi 4 \mathrm{~mm}$

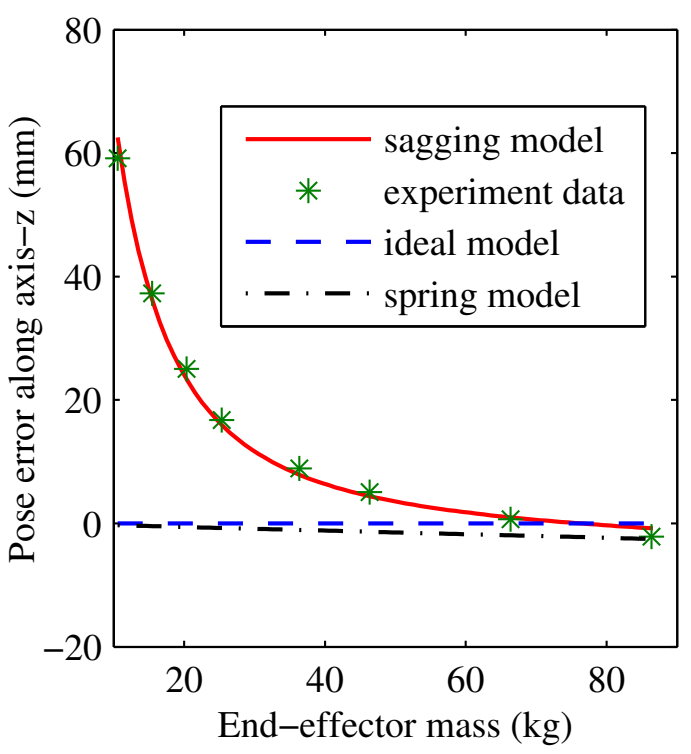

(b) Cable diameter: $\phi 8 \mathrm{~mm}$

Fig. 4.4 Effect of external load on the static pose error of the 6-cable CDPR prototype.

The effect of external loads on the static pose error of the studied CDPR with $\phi 4 \mathrm{~mm}$ or $\phi 8 \mathrm{~mm}$ cables is shown in fig. 4.4. Two poses of the end-effector are considered: one is near the center of the sub-workspace $\left(x_{G}=0 \mathrm{~m}, y_{G}=0 \mathrm{~m}\right.$ and $\left.z_{G}=0.5 \mathrm{~m}\right)$, and the other is near the edge of the sub-workspace $\left(x_{G}=3 \mathrm{~m}, y_{G}=0 \mathrm{~m}\right.$ and $\left.z_{G}=0.5 \mathrm{~m}\right)$, where the rotational angles around axis- $x_{G}, y_{G}$, and $z_{G}$ are approximate to zero.

In these two figures, the green stars represent the experimental results and the color lines represent the simulation results by the proposed models, where:

- The red solid lines represent the results calculated by the sagging cable model, where both the cable elasticity and the effect of cable weight on the static cable profile are considered.

- The black dash-dot lines represent the results calculated by the spring cable model, where only axial cable elasticity is considered. 
- The blue dashed lines represent the results calculated by the ideal cable model, where cable are modeled as massless straight lines without elongation. Because the ideal cable model is regarded as a reference in the definition of pose error in section 3.2.2, the pose error obtained by the ideal cable model is always zero.

From the experimental and the simulation results presented in fig. 4.4, the following discussion can be made:

Firstly, it is shown that the experimental data are quite close to the computational data obtained by the sagging cable model, which demonstrates the validity of the sagging cable model and the proposed static stiffness model of CDPRs.

In addition, the variation of the static pose error with the external load can be used to evaluate the static stiffness of CDPRs. As we can see from fig. 4.4:

- The variation of the pose error calculated by the spring cable model is constant and it does not change with the external load. This means that the static stiffness of CDPRs with spring cable model is constant and independent with external load. Because the spring cable model only considers the axial cable elasticity which does not change within the linear elastic range.

- However, the variation of the pose error computed by the sagging cable model is strongly non-linear and it varies significantly with the external load. This means that the static stiffness of CDPRs with sagging cable model depends on the external load.

- When the external load is small, the variation of the pose error with the external load is quite big, which indicates that the robot stiffness is low. Meanwhile, a big difference between the pose error calculated by the sagging cable model and that by the spring cable model can be found, which implies that the cable sag is significant and that the sag-introduced flexibility is the main source of the robot stiffness. In this case, the sagging cable model should be used to have a good prediction of the pose of the end-effector, while the spring cable without considering cable sag will lead to a big error.

- After the external load exceeds a certain value, the variation of the pose error with the external load becomes gentle, and trends to be coincident with the results obtained by the spring cable model. This means that cable sag becomes negligible and the robot stiffness is mainly caused by the axial cable elasticity. In this situation, both the sag cable model and the spring cable model have a good evaluation for the static stiffness of CDPRs. 
The above simulations are quite useful for the design and operation of CDPRs, especially for the pick-and-place applications. When the end-effector is unloaded, cable sag is significant and the static stiffness of CDPRs is low. To assure a good positioning accuracy, the sagging cable model should be used in the controller. After cargoes are loaded on the end-effector, cable sag is reduced and robot stiffness improves. Once the total external load exceeds a threshold value, sag-introduced pose error can be neglected, and the simpler spring cable model is enough to ensure a good position accuracy. Furthermore, the threshold value can be calculated by the above simulations.

It should be noted that the threshold value is not constant. It is associated with cable parameters, end-effector poses, accuracy requirements, etc. For example, as shown in fig. $4.4 \mathrm{a}$, the threshold value is about $50 \mathrm{~kg}$ for the studied CDPR with $\phi 4 \mathrm{~mm}$ cables when the end-effector is at the center of the sub-workspace, while this threshold value raises to 70 $\mathrm{kg}$ (fig. 4.4b) for the studied CDPR with $\phi 8 \mathrm{~mm}$ cables when the end-effector is at the edge of the sub-workspace. To sum up, the threshold value of the external load is an important parameter that is closely associated with the level of cable sag. It trends to increase when CDPRs use heavier cables and move to the edge of workspaces. The above simulations can be used to obtain the threshold value for any new designed CDPR.

\subsection{Modal experiments and dynamic stiffness analysis}

The objectives of the modal experiments are to verify the dynamic cable model and the proposed dynamic stiffness model of CDPRs by:

- Identifying the natural frequencies of the driven-cables and of the end-effector at certain poses in the workspace;

- Studying the effects of cable vibration on the dynamic behaviors of CDPRs.

To achieve the above objectives, the 8-cable CDPR CoGiRo [Lamaury 2013] is used in the dynamic experiments. The static cable forces are firstly verified. Then experimental modal analysis is made by using a dynamic shaker. The Frequency Response Functions (FRFs) of the driving cables and the end-effector under a harmonic excitation are then obtained. The cable and the end-effector vibration and their coupling are depicted by these FRF plots. The dynamic cable model and the dynamic stiffness model of CDPRs are validated through the comparison of the experimental and the simulation results. 


\subsubsection{Verification of the static cable forces}

As explained in section 2.2, the static cable forces are important parameters of the dynamic cable model, which have a significant effect on the cable vibration. In order to guarantee the accuracy of the modal analysis, the static cable forces are then firstly verified experimentally.

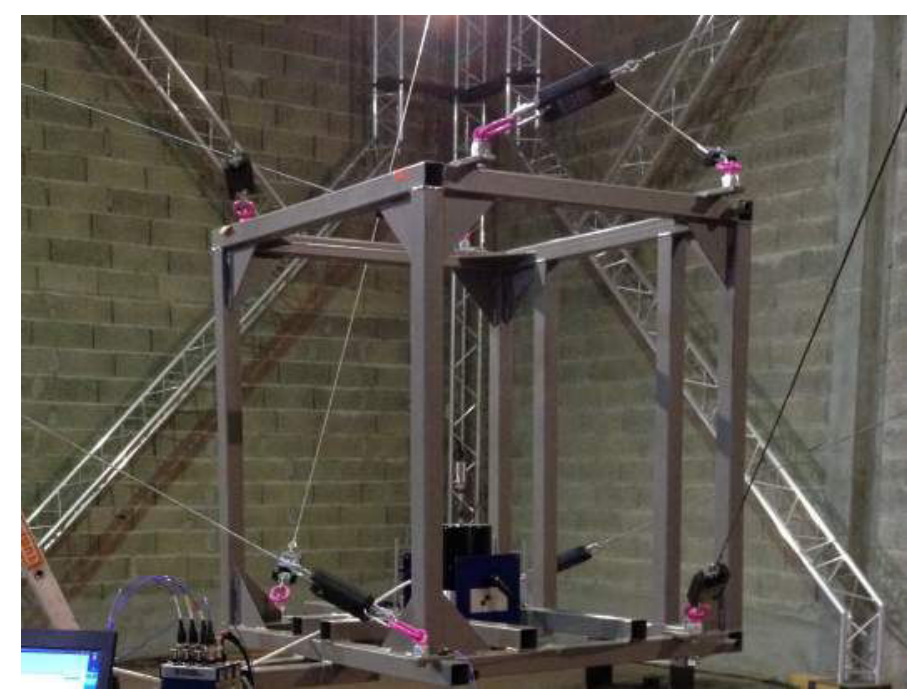

Fig. 4.5 Measurement of the static cable forces of the CoGiRo

As shown in fig. 4.5, eight dynamometers (fig. A.3a in the Appendix) are used to connect the cable ends and the attachment points on the end-effector. Through the commands given by the controller, the end-effector is moved to the following poses in the workspace, respectively:

1) $1^{\text {st }}$ pose: horizontal center pose at $x=0.012 \mathrm{~m} ; y=0.0697 \mathrm{~m} ; z=1.219 \mathrm{~m} ; \alpha, \beta, \gamma=0^{\circ}$;

2) $2^{\text {nd }}$ pose: middle edge horizontal pose at $x=4.012 \mathrm{~m} ; y=0.0697 \mathrm{~m} ; z=1.219 \mathrm{~m}$; $\alpha, \beta, \gamma=0^{\circ}$

3) $3^{r d}$ pose: horizontal corner pose at $x=4.012 \mathrm{~m} ; y=2.930 \mathrm{~m} ; z=1.219 \mathrm{~m} ; \alpha, \beta, \gamma=0^{\circ}$;

4) $4^{\text {th }}$ pose: inclined center pose at $x=0.012 \mathrm{~m} ; y=0.0697 \mathrm{~m} ; z=1.219 \mathrm{~m} ; \alpha=23^{\circ}$; $\beta, \gamma=0^{\circ}$.

For each pose, the readings of each dynamometer are recorded after all the cables are stable. Table 4.4 presents the static cable forces measured at several different poses of the end-effector in the workspace.

The cable forces measured by the dynamometers are compared with those calculated by simulations. As is shown, the cable forces measured by dynamometer have a little difference 
Table 4.4 Static forces of the driving cables measured at different poses of the end-effector in the workspace

\begin{tabular}{|c|c|c|c|c|c|c|c|c|}
\hline Pose of the end-effector & \multicolumn{10}{|c|}{ horizontal center pose } \\
\hline Cable sequence number & $1^{\text {st }}$ & $2^{\text {nd }}$ & $3^{\text {rd }}$ & $4^{\text {th }}$ & $5^{\text {th }}$ & $6^{\text {th }}$ & $7^{\text {th }}$ & $8^{\text {th }}$ \\
\hline Measured cable force (N) & 343 & 331 & 372 & 353 & 307 & 338 & 369 & 355 \\
\hline Cable force in simulation (N) & 344 & 340 & 353 & 351 & 334 & 358 & 354 & 349 \\
\hline Absolute difference (N) & 1 & 9 & 19 & 2 & 28 & 19 & 15 & 5 \\
\hline Relative difference (\%) & 0 & 3 & 5 & 1 & 9 & 6 & 4 & 1 \\
\hline
\end{tabular}

\begin{tabular}{|c|c|c|c|c|c|c|c|c|}
\hline Pose of the end-effector & \multicolumn{8}{|c|}{ middle edge horizontal pose } \\
\hline Cable sequence number & $1^{\text {st }}$ & $2^{\text {nd }}$ & $3^{\text {rd }}$ & $4^{\text {th }}$ & $5^{\text {th }}$ & $6^{\text {th }}$ & $7^{\text {th }}$ & $8^{\text {th }}$ \\
\hline Measured cable force (N) & 207 & 189 & 226 & 227 & 383 & 413 & 429 & 394 \\
\hline Cable force in simulation (N) & 208 & 198 & 211 & 202 & 394 & 429 & 427 & 396 \\
\hline Absolute difference (N) & 1 & 9 & 16 & 25 & 11 & 16 & 2 & 2 \\
\hline Relative difference (\%) & 1 & 5 & 7 & 11 & 3 & 4 & 0 & 1 \\
\hline
\end{tabular}

\begin{tabular}{|c|c|c|c|c|c|c|c|c|}
\hline Pose of the end-effector & \multicolumn{9}{|c|}{ horizontal corner pose } \\
\hline Cable sequence number & $1^{\text {st }}$ & $2^{\text {nd }}$ & $3^{\text {rd }}$ & $4^{\text {th }}$ & $5^{\text {th }}$ & $6^{\text {th }}$ & $7^{\text {th }}$ & $8^{\text {th }}$ \\
\hline Measured cable force (N) & 277 & 284 & 146 & 123 & 213 & 224 & 507 & 491 \\
\hline Cable force in simulation (N) & 228 & 214 & 201 & 192 & 167 & 162 & 554 & 525 \\
\hline Absolute difference (N) & 50 & 71 & 55 & 68 & 46 & 63 & 47 & 34 \\
\hline Relative difference (\%) & 18 & 25 & 37 & 55 & 21 & 28 & 9 & 7 \\
\hline
\end{tabular}

\begin{tabular}{|c|c|c|c|c|c|c|c|c|}
\hline Pose of the end-effector & \multicolumn{10}{|c|}{ inclined center pose } \\
\hline Cable sequence number & $1^{\text {st }}$ & $2^{\text {nd }}$ & $3^{\text {rd }}$ & $4^{\text {th }}$ & $5^{\text {th }}$ & $6^{\text {th }}$ & $7^{\text {th }}$ & $8^{\text {th }}$ \\
\hline Measured cable force (N) & 363 & 300 & 372 & 324 & 255 & 347 & 397 & 481 \\
\hline Cable force in simulation (N) & 380 & 318 & 342 & 339 & 263 & 477 & 364 & 286 \\
\hline Absolute difference (N) & 18 & 18 & 30 & 14 & 9 & 130 & 33 & 195 \\
\hline Relative difference (\%) & 5 & 6 & 8 & 4 & 3 & 37 & 8 & 41 \\
\hline
\end{tabular}

with these by simulation at the first two poses in table 4.4. All the relative differences are smaller than $9 \%$ and $11 \%$ respectively. However the correlation is not good for the last two poses in table 4.4. Relative difference is up to $37 \%$ and $55 \%$ respectively. In addition, the measured cable forces are also compared to these in the CoGiRo controller. Similar results are found: the correlation is good for the first two poses in table 4.4, bad for the last two poses in table 4.4. The reason is still unknown and further investigations should be made to conclude. As the cable forces are important parameters for vibration analysis, the following experiments will only use the first two poses in table 4.4. 


\subsubsection{Experimental setup}

In this section, an electrodynamic shaker is used to generate an harmonic excitation. FRF can be calculated and plotted according to the response of the accelerometer and the force sensor. Then different natural frequencies can be identified by analyzing the resonances in the FRF plot.
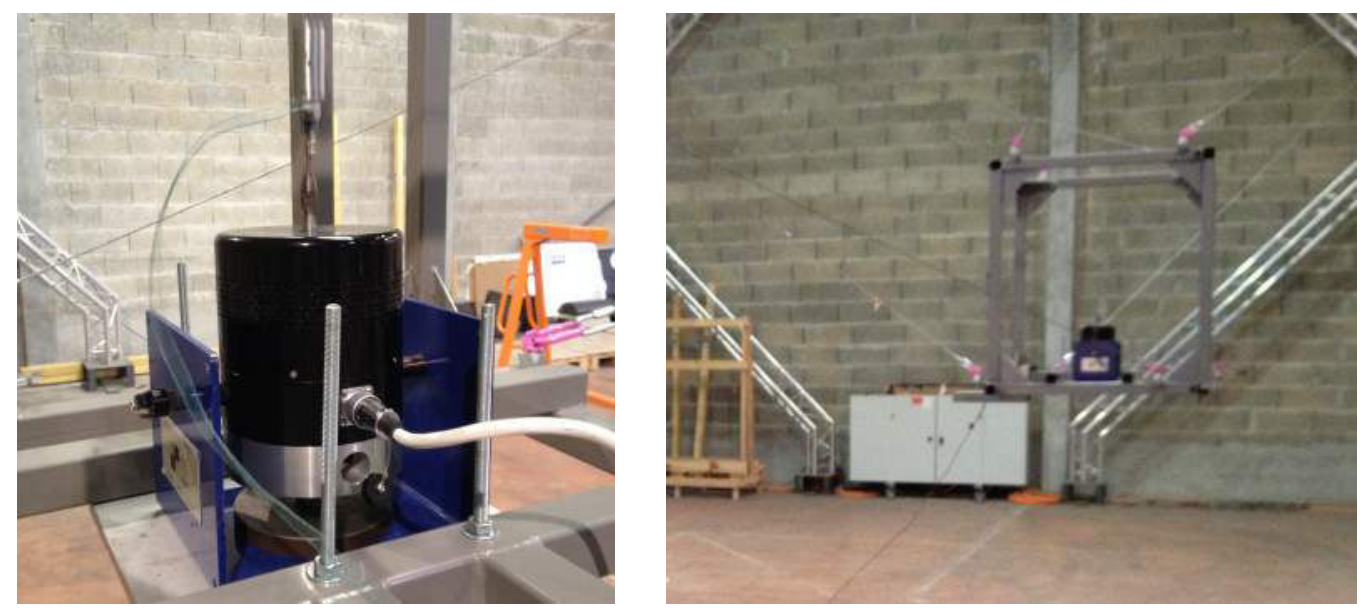

Fig. 4.6 Modal analysis of the CoGiRo by the shaker

The experimental setup is shown in section 4.3.2. The shaker is mounted on the endeffector. A small mass block is fixed to the mobile stick of the shaker. A force sensor lies between the mass block and the mobile stick. Thus, the shaker can deliver a vertical force to the end-effector. The force is proportional to the acceleration amplitude of the mass block, and can be measured by the force sensor. A triaxial accelerometer is fixed on the platform. It is used to obtain the response of the end-effector along three mutually perpendicular directions. Several other accelerometers are fixed along the cables. The weight of the accelerometer is $5.8 \mathrm{~g}$. Compared to the linear weight of the cables $(67 \mathrm{~g} / \mathrm{m}$ for $\phi 4$ $\mathrm{mm}$ cable), its weight can be negligible.

During the experiment, the end-effector is firstly moved to a certain pose in the workspace $\left(x=0.012 \mathrm{~m} ; y=0.0697 \mathrm{~m} ; z=1.219 \mathrm{~m} ; \alpha, \beta, \gamma=0^{\circ}\right)$. After all the cables and the endeffector are stable, a harmonic excitation is generated by the shaker. This harmonic excitation has a fixed frequency changed step by step. The step size is $0.05 \mathrm{~Hz}$. At each step, there is a stabilization time of $8 \mathrm{~s}$, and a measuring time of $8 \mathrm{~s}$. As the low-order natural frequencies are important to evaluate the dynamic stiffness of CoGiRo, the dynamic experiments mainly focus on the low frequency. However, the electro-dynamic shaker is not suitable for the dynamic test below $2 \mathrm{~Hz}$. Therefore, the examined frequency range here is $2 \sim 20 \mathrm{~Hz}$. Then 
the end-effector is moved to another pose in the workspace $(x=4.012 \mathrm{~m} ; y=0.0697 \mathrm{~m}$; $\left.z=1.219 \mathrm{~m} ; \alpha, \beta, \gamma=0^{\circ}\right)$, and the previous step is repeated.

\subsubsection{Experimental results and discussions}

\section{Natural frequencies of the cables}

The cable dynamic is firstly analyzed. FRFs are calculated referring to the cable acceleration responses and the excitation force. Figure 4.7 a presents the FRF plot of the $5^{\text {th }}$ cable, when the end-effector is at the pose near the center of the workspace. As is shown, the horizontal axis presents frequencies within a range of $2 \sim 20 \mathrm{~Hz}$. The vertical axis presents the amplitude of the FRF. As the unit of acceleration is $g$ and the unit of force is $\mathrm{N}$, the unit of the amplitude is $g / \mathrm{N}$. Several peaks of the amplitude can be found in this FRF plot. These peaks are the resonances of the system. From these resonances, cable natural frequencies can be identified, which are listed in the first line of table 4.5 .

Table 4.5 Cable natural frequencies identified by experiments and simulations at the pose near the center of the workspace $\left(x=0.012 \mathrm{~m} ; y=0.0697 \mathrm{~m} ; z=1.219 \mathrm{~m} ; \alpha, \beta, \gamma=0^{\circ}\right)$

\begin{tabular}{|c|c|c|c|c|c|}
\hline Data sources & \multicolumn{5}{|c|}{ Identified cable natural frequencies } \\
\hline Experiment (Hz) & 3.8 & 7.3 & 10.9 & 14.5 & 18.0 \\
\hline Simulation by DSM (Hz) & 3.6 & 7.1 & 10.7 & 14.3 & 17.8 \\
\hline Relative difference (\%) & 4.0 & 2.3 & 1.8 & 1.7 & 0.7 \\
\hline
\end{tabular}

Table 4.6 Cable natural frequencies identified by experiments and simulations at the pose near the edge of the workspace $\left(x=4.012 \mathrm{~m} ; y=0.0697 \mathrm{~m} ; z=1.219 \mathrm{~m} ; \alpha, \beta, \gamma=0^{\circ}\right)$

\begin{tabular}{|c|c|c|c|}
\hline Data sources & \multicolumn{3}{|c|}{ Identified cable natural frequencies } \\
\hline Experiment (Hz) & 5.2 & 10.4 & 15.6 \\
\hline Simulation by DSM (Hz) & 5.4 & 10.7 & 15.7 \\
\hline Relative difference (\%) & 3.8 & 2.5 & 0.3 \\
\hline
\end{tabular}

Simulation results by DSM method are presented in fig. $4.7 \mathrm{~b}$. As is shown, the vertical axis presents the amplitude variation of the trace of the principal cable dynamic stiffness matrix. The cable vibration is introduced in the modeling of the CDPR by variation of the dynamic stiffness matrix through the frequency range. As we can see in fig. $4.7 \mathrm{~b}$, several 


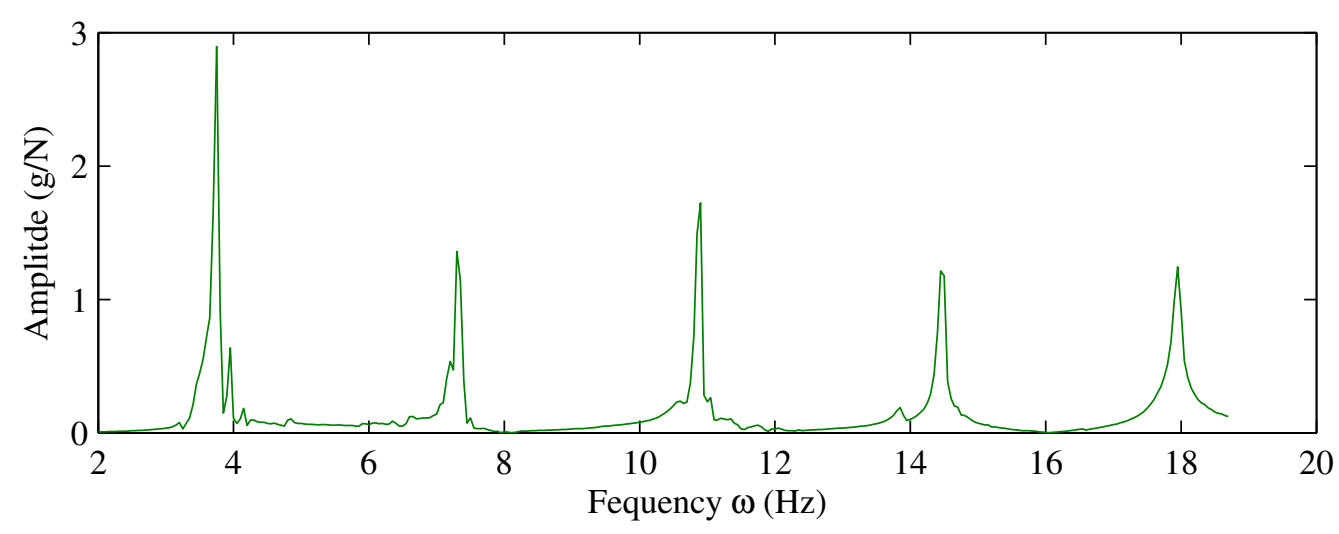

(a) Frequency response function between the cable acceleration response and the excitation force

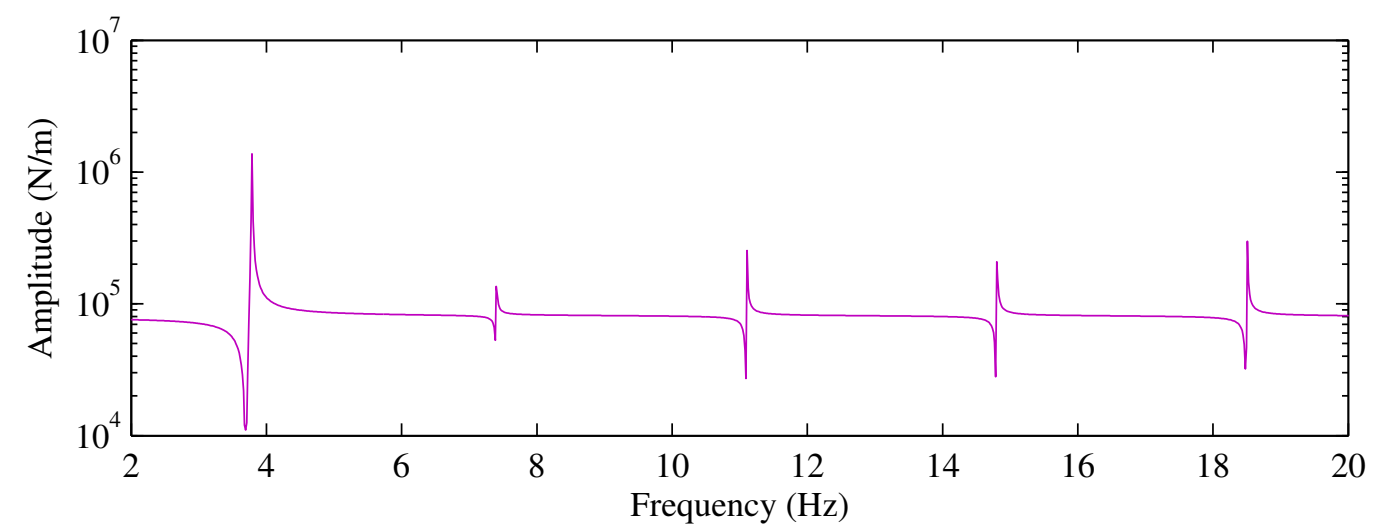

(b) Amplitude variation of the trace of the principal cable dynamic stiffness matrix by DSM method

Fig. 4.7 Experimental and simulation results of the vibration analysis of the $5^{\text {th }}$ cable at the pose near the center of the workspace $\left(x=0.012 \mathrm{~m} ; y=0.0697 \mathrm{~m} ; z=1.219 \mathrm{~m} ; \alpha, \beta, \gamma=0^{\circ}\right)$

peaks appear. These peaks correspond to the cable natural frequencies, which are listed in the second line of table 4.5. Similar experiments and simulations are made at another pose near the edge of the workspace. Results are presented in fig. 4.8 and table 4.6.

As we can see from tables 4.5 and 4.6, the cable natural frequencies identified by experimental modal analysis are close to these obtained by the DSM method. The relative differences between simulation and experiment are all less than 5 percent. This is a good validation of the DSM method in the dynamic cable modeling. It should be noted that similar measurements are made for another two cables, L4 and L6. Conclusions are the same. For the sake of brevity, only the results of cable L5 are given here. 


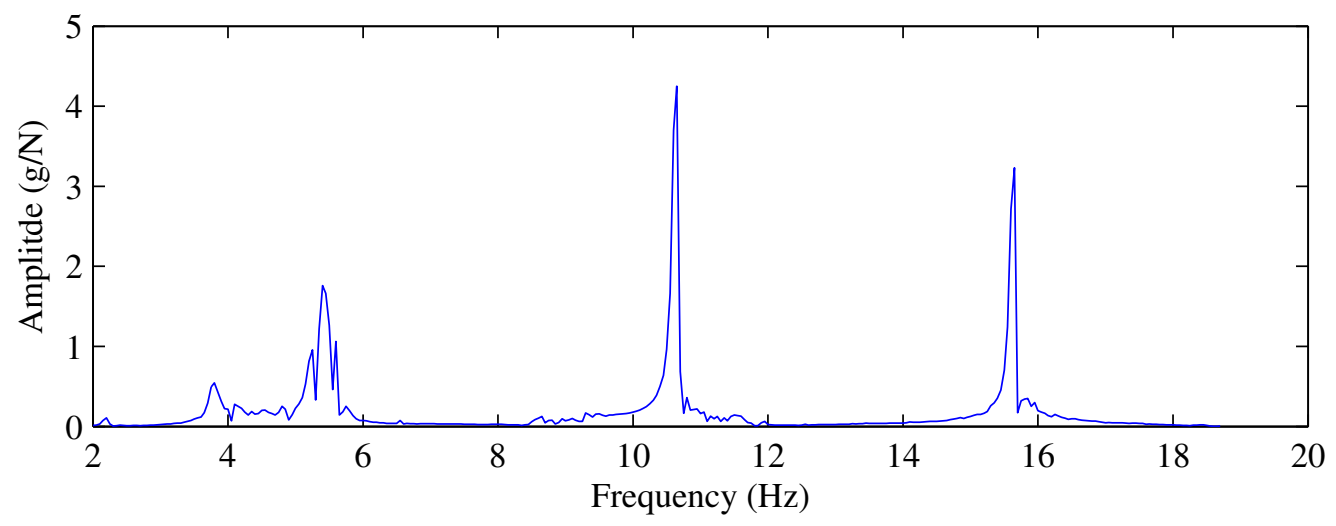

(a) Frequency response function between the cable acceleration response and the excitation force

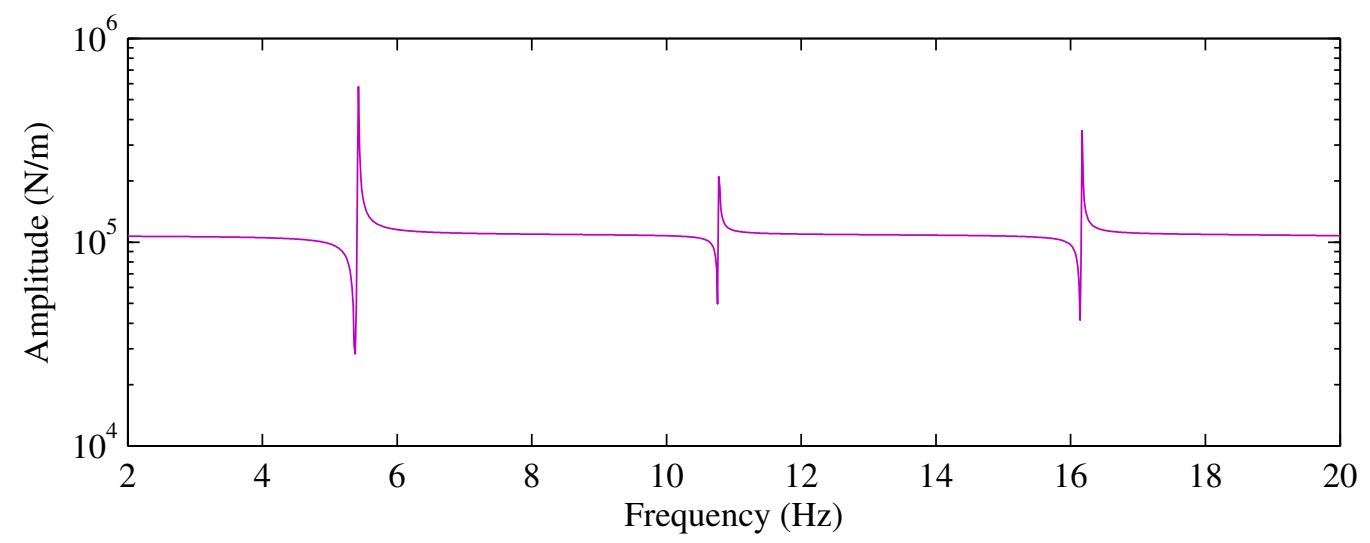

(b) Amplitude variation of the trace of the principal cable dynamic stiffness matrix by DSM method

Fig. 4.8 Experimental and simulation results of the vibration analysis of the $5^{\text {th }}$ cable at the pose near the edge of the workspace $\left(x=4.012 \mathrm{~m} ; y=0.0697 \mathrm{~m} ; z=1.219 \mathrm{~m} ; \alpha, \beta, \gamma=0^{\circ}\right)$

\section{Natural frequencies of the CDPR}

Figure 4.9 presents the experimental and simulation results of the vibration analysis of the end-effector at the pose near the center of the workspace. The four plots allow to analyze the system behavior and identify the natural frequencies.

- Figure 4.9a depicts the experimental FRF plot referring to the acceleration response of the end-effector along $x, y, z$-axis and the excitation force along $z$-axis. The maximum of the amplitude corresponds to the modes of the 8 driven cables and the modes of the end-effector.

- Figure $4.9 \mathrm{~b}$ shows the amplitude of the determinant of $\omega^{2} \mathbf{E}-\mathbf{M}^{-1} \mathbf{K}_{\mathrm{DSM}}$, where $\mathbf{M}$ is the $6 \times 6$ mass matrix of the end-effector, $\mathbf{K}_{\mathrm{DSM}}$ is the dynamic stiffness matrix (complex values) of the end-effector and $\mathbf{E}$ is the $6 \times 6$ identity matrix. As explained 


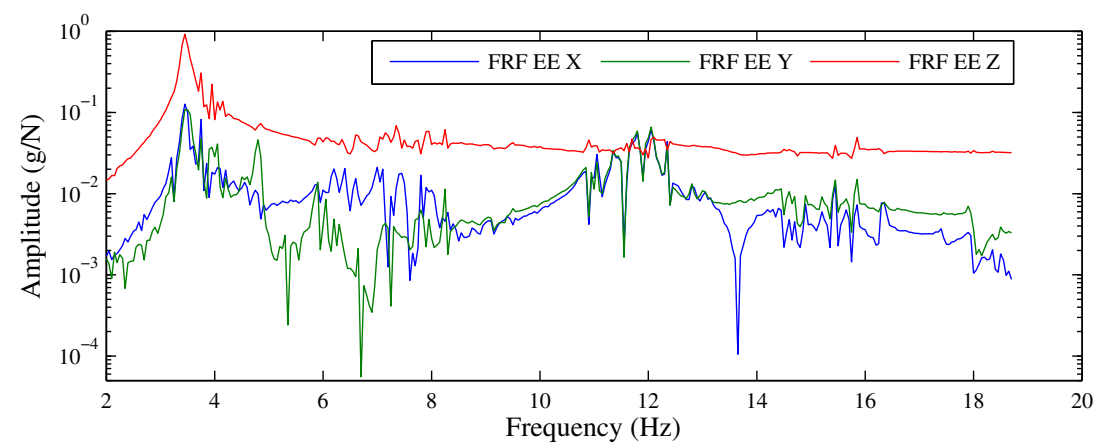

(a) Frequency response function between the acceleration response of the endeffector along $x, y, z$-axis and the excitation force along $z$-axis

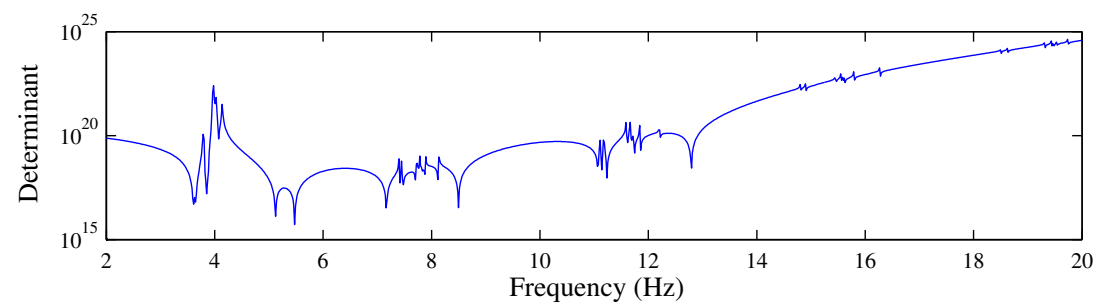

(b) Amplitude of the determinant of $\omega^{2} \mathbf{E}-\mathbf{M}^{-1} \mathbf{K}_{\mathrm{DSM}}$ (simulation result considering cable dynamics by DSM method)

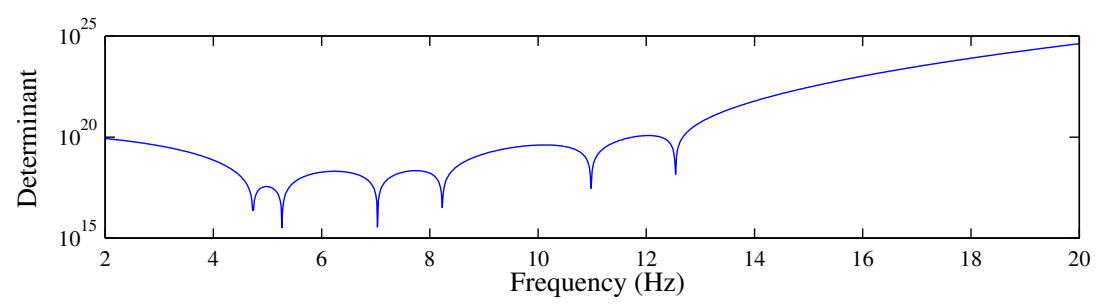

(c) Amplitude of the determinant of $\omega^{2} \mathbf{E}-\mathbf{M}^{-1} \mathbf{K}_{\text {static }}$ (simulation result neglecting cable dynamics [Kozak+ 2006])

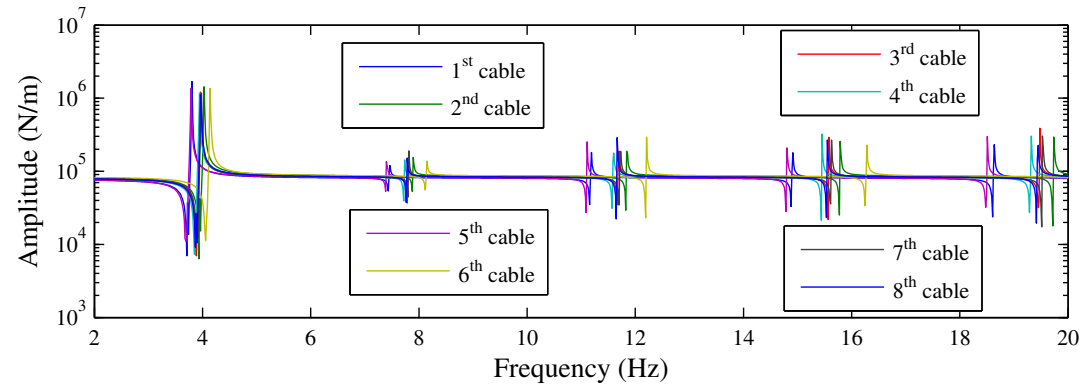

(d) Amplitude variation of the trace of the cable dynamic stiffness matrix by DSM method

Fig. 4.9 Experimental and simulation results of the vibration analysis of the end-effector at the pose near the center of the workspace $(x=0.012 \mathrm{~m} ; y=0.0697 \mathrm{~m} ; z=1.219 \mathrm{~m}$; $\left.\alpha, \beta, \gamma=0^{\circ}\right)$ 


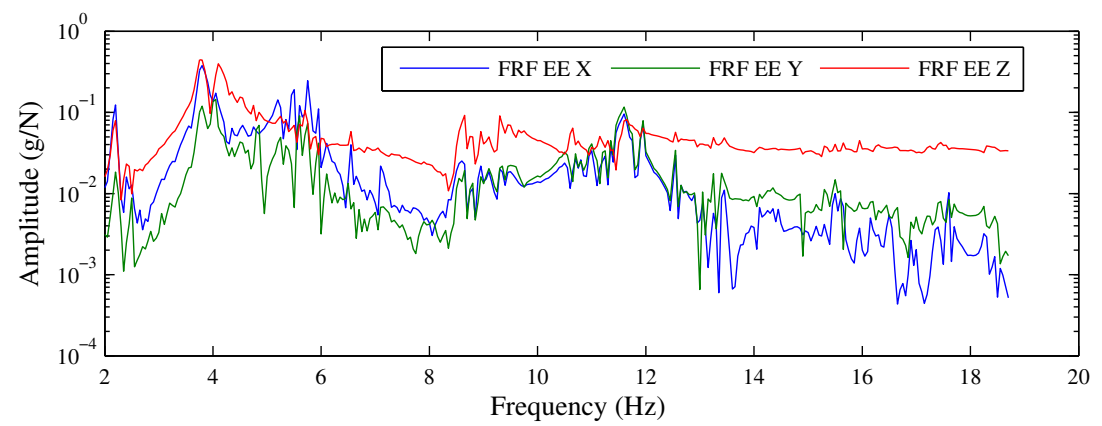

(a) Frequency response function between the acceleration response of the endeffector along $x, y, z$-axis and the excitation force along $z$-axis

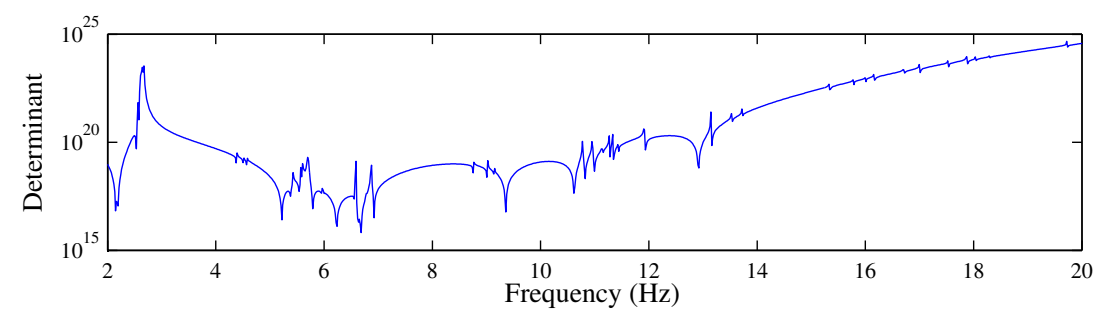

(b) Amplitude of the determinant of $\omega^{2} \mathbf{E}-\mathbf{M}^{-1} \mathbf{K}_{\mathrm{DSM}}$ (simulation result considering cable dynamics by DSM method)

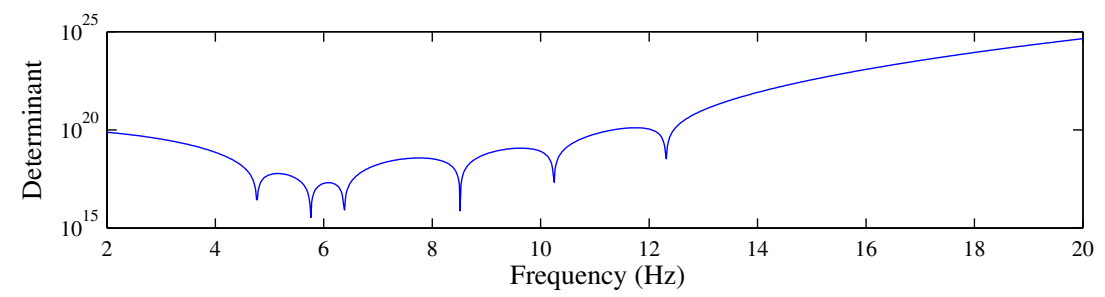

(c) Amplitude of the determinant of $\omega^{2} \mathbf{E}-\mathbf{M}^{-1} \mathbf{K}_{\text {static }}$ (simulation result neglecting cable dynamics [Kozak+ 2006])

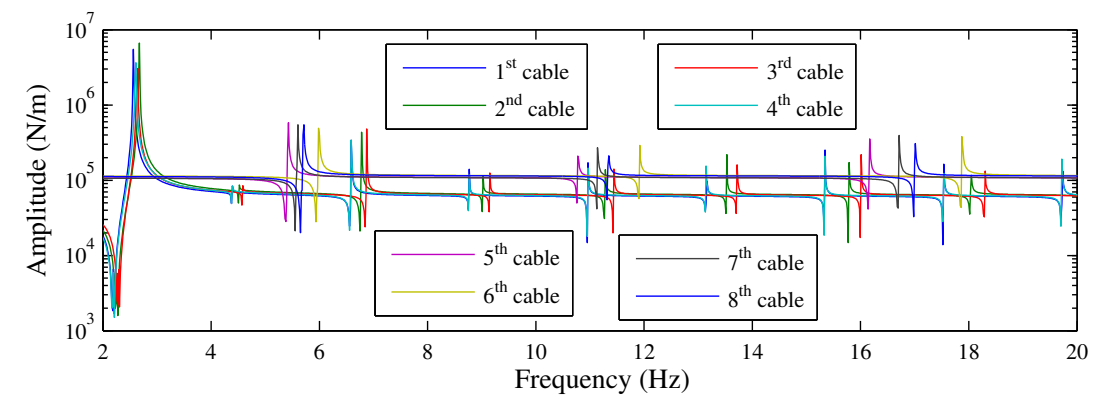

(d) Amplitude variation of the trace of the cable dynamic stiffness matrix by DSM method

Fig. 4.10 Experimental and simulation results of the vibration analysis of the end-effector at the pose near the edge of the workspace $(x=4.012 \mathrm{~m} ; y=0.0697 \mathrm{~m} ; z=1.219 \mathrm{~m}$; $\left.\alpha, \beta, \gamma=0^{\circ}\right)$ 
in section 3.3.1, cable dynamics is considered in $\mathbf{K}_{\mathrm{DSM}}$. The amplitude variation of the determinant of $\omega^{2} \mathbf{E}-\mathbf{M}^{-1} \mathbf{K}_{\mathrm{DSM}}$ reflects end-effector modes, cable modes, and their coupling.

- Figure 4.9c shows the amplitude of the determinant of $\omega^{2} \mathbf{E}-\mathbf{M}^{-1} \mathbf{K}_{\text {static }}$, where $\mathbf{K}_{\text {static }}$ is the static stiffness matrix of the end-effector [Kozak+ 2006]. Cable dynamics is neglected in $\mathbf{K}_{\text {static }}$. The resonances in fig. 4.9c correspond to the 6 modes of the end-effector.

- Figure 4.9d shows the amplitude variations of the trace of the cable dynamic stiffness matrix for each of the 8 driven cables. The maximum values correspond to the cable modes.

It should be noticed that FRF is not used to present the simulation results in figs. 4.9 and 4.10. In fact, there is a strong coupling of cable modes and end-effector modes in low frequencies, where a lot of energy is transfered to cables due to the harmonic excitation used in that modal analysis (16 s by frequency step). The amplitudes of simulated and measured FRF cannot be directly compared because the modeling approach used for the damping is too restrictive (only a constant viscous damping parameter for the cable modeling) and do not depict the reality. Through a sensitivity analysis not presented here, we expect a strong non-linearity of the damping through the frequency and a strong part of friction damping brought by the joints between the cables and the end-effector. In a first approach, it does not affect the main objective of the modal analysis which is to identify the natural frequencies of the system. But this point should be investigated in future works to be able to predict the energy dissipation.

Instead of FRF plots, the determinant of $\omega^{2} \mathbf{E}-\mathbf{M}^{-1} \mathbf{K}$ is used to present the simulation results and identify the natural frequencies. The amplitude of determinant is calculated and plotted with logarithmic coordinates. Normally, the frequency where the determinant is zero corresponds to a natural frequency. Due to the discretization of the frequency range for time calculation convenience, it is impossible to present the zero points of determinant. Nevertheless, the minimum values correspond to the natural frequencies. Moreover, the determinant is complex number if considering cable dynamics.

From the above experimental results, following analysis and discussion can be made:

- Firstly, fig. 4.9a is compared with fig. 4.9b. As the vertical axis of these two figures have different meanings, they cannot be compared directly. However, as we explained before, the resonances corresponding to the natural frequencies could be identified in 
figs. 4.9a and 4.9b. The frequency of each resonance in fig. 4.9a is compared with the frequencies of the lowest values of the determinant in fig. $4.9 \mathrm{~b}$. As we can see, the natural frequencies in these two figures correspond well, even there exist some small differences. Experimentally, the natural frequencies seems to be globally a little bit lower (about $0.5 \mathrm{~Hz}$ ); It is due to the modeling and experimental errors. In the experimental setup, metal rings are used as joints to connect the cable and the end-effector. However it is assumed in the simulation that the cable end is fixed on the end-effector. Another reason could be the inertia parameters of the end-effector used in the simulations. These parameters have been obtained by CAD model without considering the welds.

- Secondly, fig. $4.9 \mathrm{~b}$ is compared with fig. 4.9c. These two figures have similar definition of the vertical axis. They are both the determinant of $\omega^{2} \mathbf{E}-\mathbf{M}^{-1} \mathbf{K}$. The difference lies in the stiffness matrix computation. In fig. $4.9 \mathrm{~b}$, cable dynamics are considered through the dynamic stiffness matrix $\mathbf{K}_{\mathrm{DSM}}$. While in fig. $4.9 \mathrm{c}$, the static stiffness matrix $\mathbf{K}_{\text {static }}$ totally neglects cable dynamics. Thus the peaks in fig. $4.9 \mathrm{~b}$ correspond to the natural frequencies of the global system, considering the modes of the cables and the rigid-body modes of the end-effector suspended on the stiffness of the cables. While the peaks in fig. 4.9c represents only the rigid-body modes of the end-effector suspended on the static stiffness of the cables (considering only elasticity and sag). It is indicated that the proposed DSM method is more accurate to predict the experimental results.

As shown in fig. 4.9b, there are some dense peaks around 4, 8, 12, 16 and $19 \mathrm{~Hz}$. If taking a look at fig. $4.9 \mathrm{~d}$, we can find out that these peaks have the same natural frequencies with the cable peaks in fig. $4.9 \mathrm{~d}$. Therefore, these peaks in fig. $4.9 \mathrm{~b}$ are caused by cable dynamics. There are also some relatively separated peaks in fig. $4.9 \mathrm{~b}$, such as at 5.1, 5.5, 7.2, 8.5 and $12.8 \mathrm{~Hz}$. These peaks correspond to the rigid body modes of the end-effector on the global cable stiffness. In conclusion, by comparing figs. $4.9 \mathrm{~b}$ to $4.9 \mathrm{~d}$, it is indicated that cable dynamics can affect the dynamic behaviors of CoGiRo by adding new resonances and changing the value of natural frequencies in a second order because the robot configurations present a low sag in the cables.

- Thirdly, it is shown that the amplitude of the first resonance of the end-effector around $4 \mathrm{~Hz}$ is much bigger than the other resonances in fig. 4.9a. If we compare fig. 4.9a with other three figures in fig. 4.9 , especially fig. $4.9 \mathrm{~d}$, we can find out this resonance with strong amplitude is due to the first mode of the 8 driven cables. The level of amplitude 
indicated that a lot of energy is transferred between cables and end-effector, especially in low frequency. In that case, cable dynamics has to be considered in the vibration analysis of CoGiRo.

Similar experiments are made at another pose near the edge of the workspace $(x=4.012$ $\mathrm{m} ; y=0.0697 \mathrm{~m} ; z=1.219 \mathrm{~m} ; \alpha, \beta, \gamma=0^{\circ}$ ). Results are presented in fig. 4.10 , through which same conclusions can be made.

\subsection{Dynamic trajectory experiments and dynamic stiffness analysis}

The major objectives of the dynamic trajectory experiments are to study the dynamic behaviors of the CDPR prototype during a trajectory, such as identifying the natural frequency of the driving cables and the end-effector, analyzing the coupling between cable dynamics and end-effector vibration. To achieve the above objectives, the 8-cable CDPR CoGiRo [Lamaury 2013] is used. Free vibration experiments at an emergency stop during a trajectory and dynamic experiments along a complete trajectory are carried out.

\subsubsection{Free vibration experiment at an emergency stop}

In this section, free vibration is analyzed to investigate the dynamic behavior of CoGiRo. An emergency stop is used to generate an impulse excitation. All the modes can be excited and each response depends on the initial conditions.

\section{Experimental setup}

The experimental setup of the free vibration of the CDPR prototype at an emergency stop is shown in section 4.4.1, and the experimental procedure is as following:

1) A triaxial accelerometer is adhered to the end-effector, and another accelerometer is adhered along the $5^{\text {th }}$ cable.

2) After the data acquisition system is activated, a command is given through the controller to move the end-effector along a certain trajectory (straight line from $x=1 \mathrm{~m} ; y=-2$ $\mathrm{m} ; z=0 \mathrm{~m} ; \alpha, \beta, \gamma=0^{\circ}$ to $\left.x=-0.5 \mathrm{~m} ; y=-0.7 \mathrm{~m} ; z=-0.25 \mathrm{~m} ; \alpha, \beta, \gamma=0^{\circ}\right)$. Then push emergency stop to lock the motor and get an impulse excitation to the end-effector at the middle point of the trajectory. This pose is quite closed to the pose in fig. 4.9. 


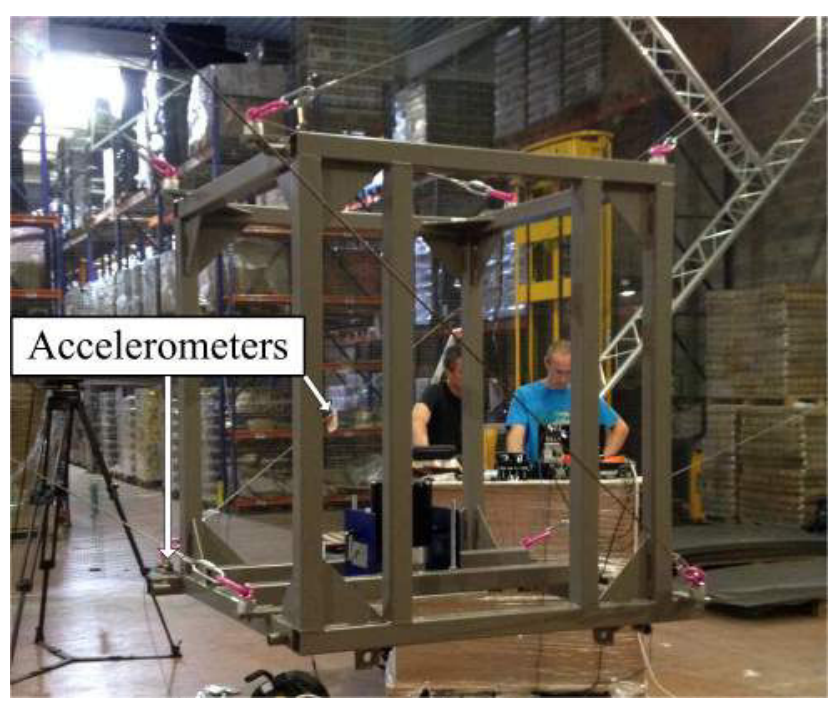

Fig. 4.11 Free vibration experiment at an emergency stop

\section{Experimental results and discussions}

As we know, classical Fourier analysis assumes that signals are periodic and infinite in time. However the signals obtained by free vibration here are of short duration and change substantially over their duration. Therefore, time-frequency analysis is used to study the signals in both time and frequency domains. According to the experimental results shown in figs. 4.12 to 4.14 , the following discussion can be made.

- Figure 4.12 presents the experimental results of the vibration analysis of the $5^{\text {th }}$ cable during a trajectory containing an emergency stop. The acceleration of the $5^{\text {th }}$ cable in time domain is shown in fig. $4.12 \mathrm{a}$, where the horizontal axis presents time and the vertical axis presents the acceleration amplitude. Obvious variations of the acceleration can be found after $6 \mathrm{~s}$, when the emergency stop is applied.

- In order to study the dynamic behavior in frequency domain, time-frequency analysis is made. Figure $4.12 \mathrm{~b}$ presents the spectrogram of the frequency response of the $5^{\text {th }}$ cable. The horizontal axis presents time and the vertical axis presents frequency. The color depicts the magnitude of the energy of the signal at the time moment $t(0 \leq t \leq 16 \mathrm{~s})$, and the frequency $f(0 \leq f \leq 25 \mathrm{~Hz})$. The spectrum color from red to blue corresponds to the energy level from strongest to weakest. From fig. $4.12 \mathrm{~b}$, obvious change in spectral content of the signal over time can be found, which corresponds to different cable modes. In fig. $4.12 \mathrm{~b}$, the cable mode around $3 \mathrm{~Hz}$ can be identified clearly. It is the first mode of the $5^{\text {th }}$ cable. This result coincides with the result of the modal analysis by shaker in section 4.3.2 (fig. 4.7). 


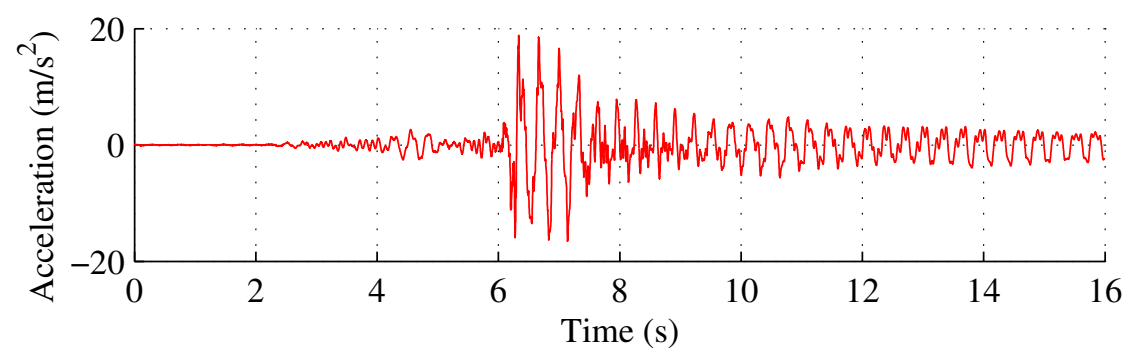

(a) Acceleration of the $5^{\text {th }}$ cable

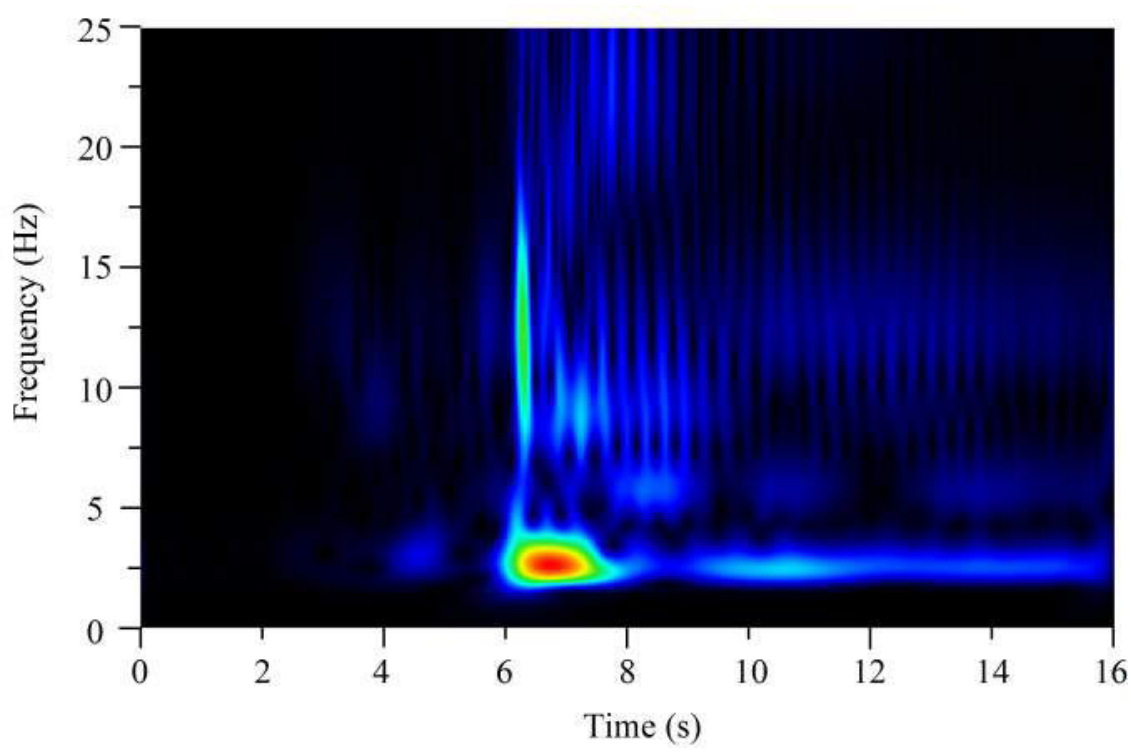

(b) Time-Frequency analysis of the $5^{\text {th }}$ cable

Fig. 4.12 Experimental results of the free vibration analysis of the $5^{\text {th }}$ cable during a trajectory containing an emergency stop

- Figures 4.13 and 4.14 present the experimental results of the free vibration analysis of the end-effector along axis- $x$ and axis- $z$ respectively. The acceleration of the endeffector along axis- $x$ and axis- $z$ in the time domain is shown in fig. 4.13a and fig. 4.14a, respectively. Obvious variations of the acceleration can be found after $6 \mathrm{~s}$, when the emergency stop is applied. Time-frequency analysis is made to study the dynamic behavior in both time and frequency domain. Figures $4.13 \mathrm{~b}$ and $4.14 \mathrm{~b}$ present the spectrograms of the frequency response of the end-effector along axis- $x$ and axis- $z$, respectively. From these two figures, obvious changes in spectral content of the signal over time can be found. These changes correspond to different modes of the endeffector. Two groups of modes can be identified clearly. One group is around $5 \mathrm{~Hz}$ in fig. $4.13 \mathrm{~b}$, and the other is around $8 \mathrm{~Hz}$ in fig. $4.14 \mathrm{~b}$. These two groups of modes 


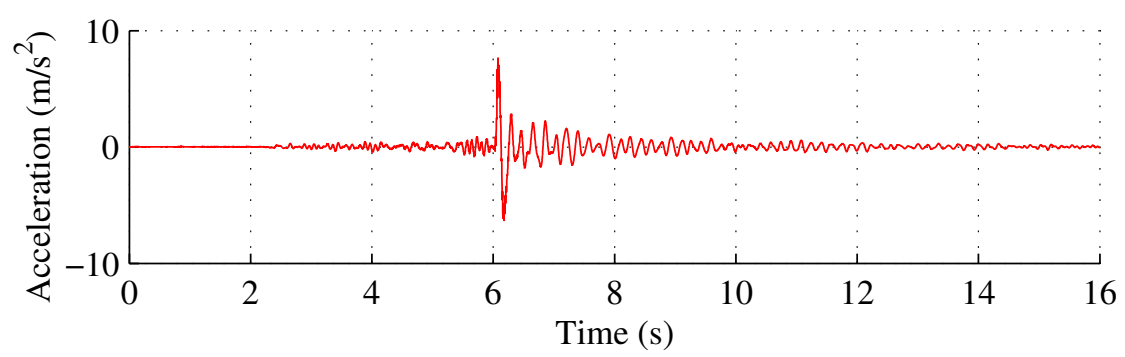

(a) Acceleration of the end-effector along axis- $x$

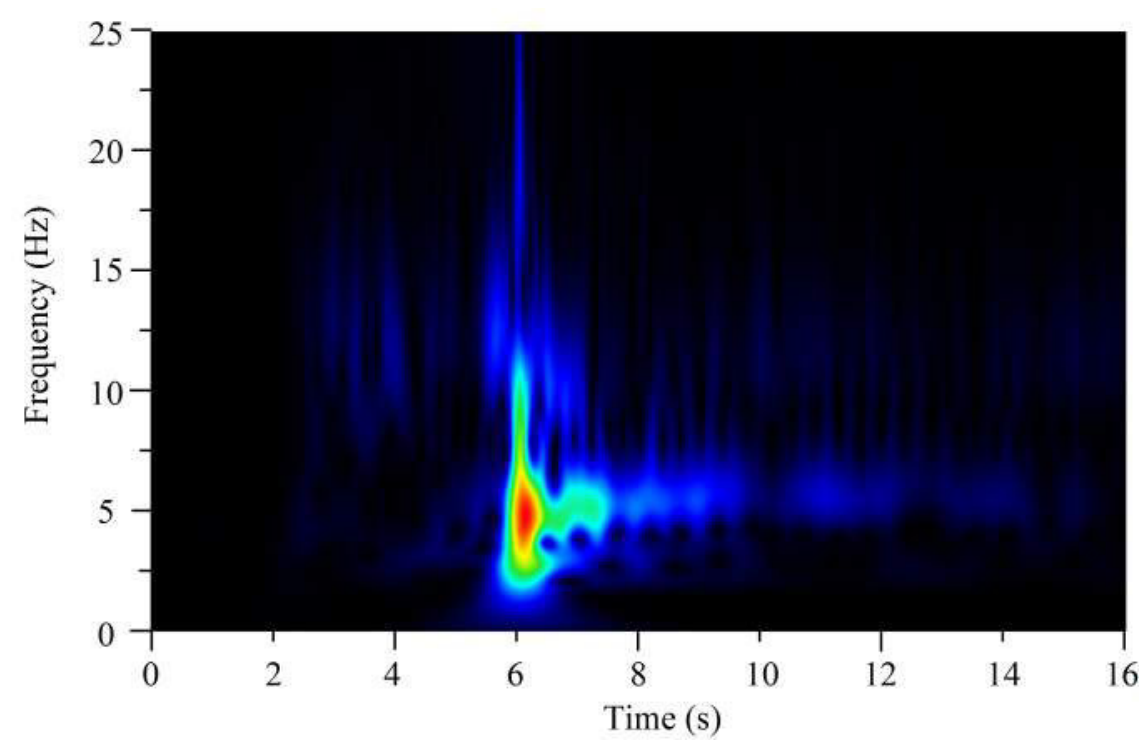

(b) Time-Frequency analysis of the end-effector along axis- $x$

Fig. 4.13 Experimental results of the free vibration analysis of the end-effector along axis- $x$ during a trajectory containing an emergency stop

coincide with the end-effector modes $(5.1,5.5,7.2,8.5 \mathrm{~Hz})$ identified in fig. 4.9b. These modes are not due to the cable dynamics. In fact, they are the rigid body modes of the end-effector. The vibration of the end-effector after an emergency stop is mainly affected by the rigid body modes of the end-effector and not by the natural frequencies of the cables, even those cables vibrate. We have to notice that the vibration of the cables should be taken into account in the computation of the dynamic stiffness matrix of the system by the DSM method even if there is little energy transfer in this case. In fact, the dynamic stiffness changes the frequency value of the rigid body modes of the end-effector (fig. 4.9). 


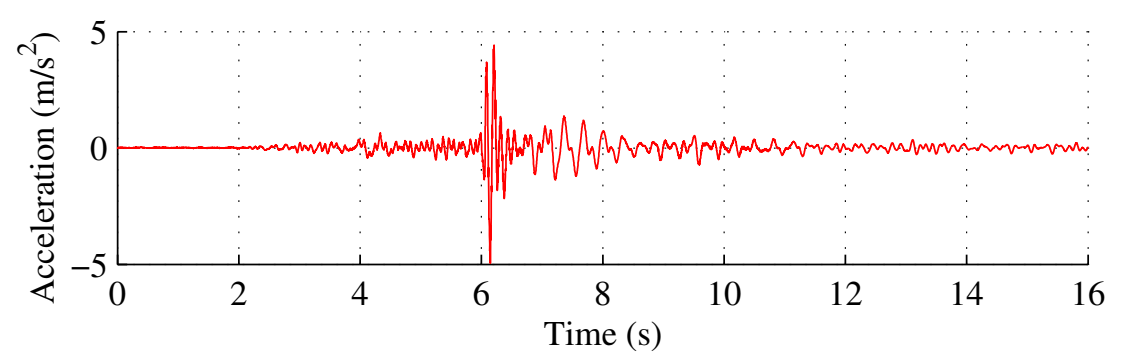

(a) Acceleration of the end-effector along axis- $z$

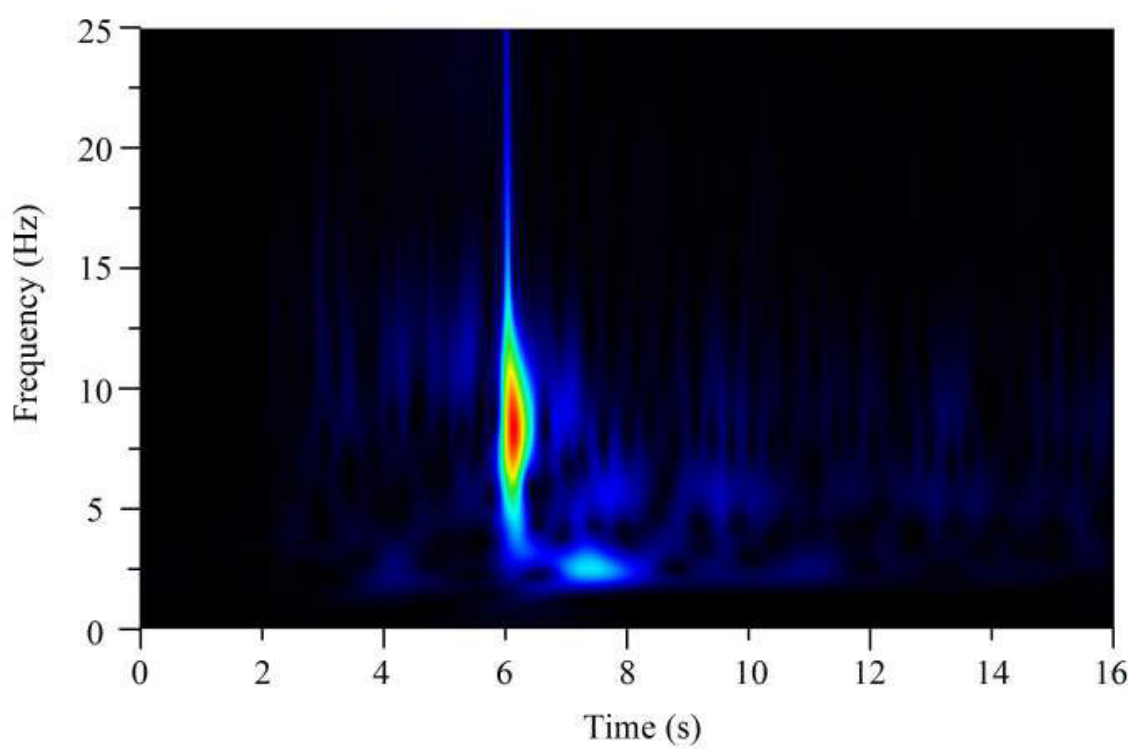

(b) Time-Frequency response of the end-effector along axis- $z$

Fig. 4.14 Experimental results of the free vibration analysis of the end-effector along axis- $z$ during a trajectory containing an emergency stop

\subsubsection{Experiment along a trajectory}

In this section, experiments are made to study the behaviors of CoGiRo during its movement along a trajectory. Experimental procedures are as following:

1) As shown in fig. 4.15, three LEDs are adhered to define the frame of the end-effector. An accelerometer is adhered along the cable, and a triaxial accelerometer is adhered to the edge of the end-effector. The Nikon metrology system K-600 is set up in front of the end-effector.

2) A command is given to the controller to move the end-effector along a certain trajectory (straight line from $x=1 \mathrm{~m} ; y=-2 \mathrm{~m} ; z=0 \mathrm{~m} ; \alpha, \beta, \gamma=0^{\circ}$ to $x=-0.5 \mathrm{~m} ; y=-0.7$ $\left.\mathrm{m} ; z=-0.25 \mathrm{~m} ; \alpha, \beta, \gamma=0^{\circ}\right)$. The time of this trajectory is set to be 30 seconds in the 

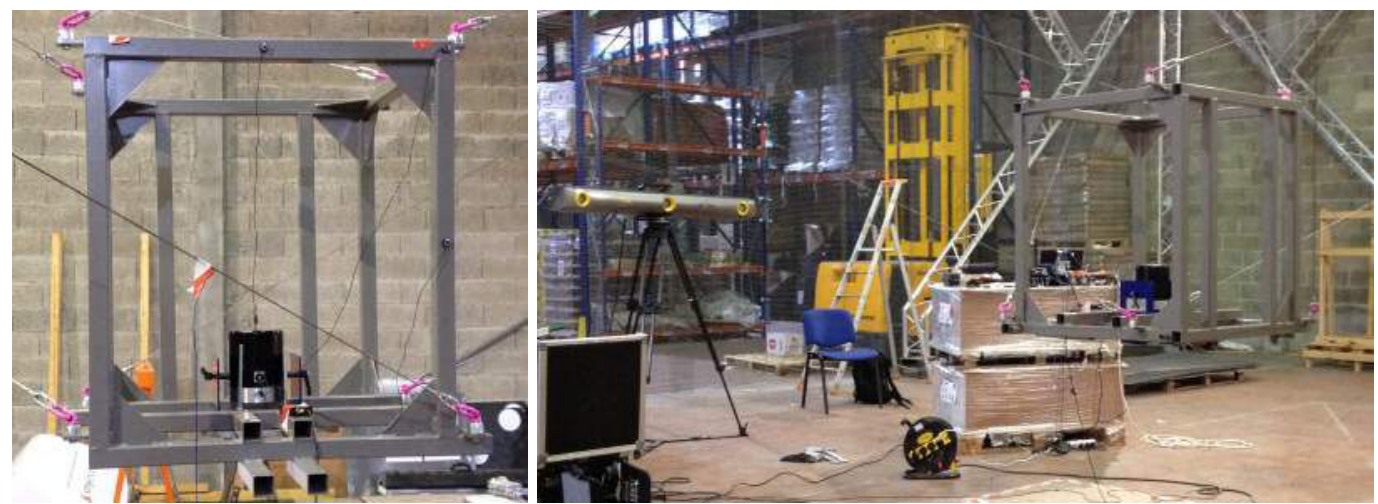

Fig. 4.15 Experimental setup of the analysis along a trajectory

controller. During the movement, the pose of the end-effector is measured by the Nikon system, and the response of the accelerometers is recorded by the NI data acquisition system.

3) The end-effector is moved to the start point of the trajectory. The time of the trajectory is reset to 10,5 , and 1.5 seconds in the controller, respectively. Then repeat the previous step.

\section{Pose error of the end-effector during the trajectory}

Firstly, the pose error of the end-effector during the trajectory is studied. The trajectory in the controller is an ideal straight line. However due to the vibration of the end-effector, the real trajectory in the experiment cannot be a straight line. The pose error of the end-effector is defined as the difference between the end-effector pose in the controller and the end-effector pose measured by the Nikon system. Figure 4.16 presents the pose error of the end-effector during the trajectory from point $x=1 \mathrm{~m} ; y=-2 \mathrm{~m} ; z=0 \mathrm{~m} ; \alpha, \beta, \gamma=0^{\circ}$ to point $x=-0.5 \mathrm{~m}$; $y=-0.7 \mathrm{~m} ; z=-0.25 \mathrm{~m} ; \alpha, \beta, \gamma=0^{\circ}$ with different trajectory time durations. Figure $4.16 \mathrm{a}$ shows the pose error along $y$-axis, and fig. $4.16 \mathrm{~b}$ shows the pose error along $z$-axis. From fig. 4.16, obvious variations of the pose error can be found. These variations are due to the vibration of the end-effector. It is also found that the faster the end-effector moves, the bigger the variation becomes. This means that the speed of the end-effector can affect the amplitude of its vibration. 


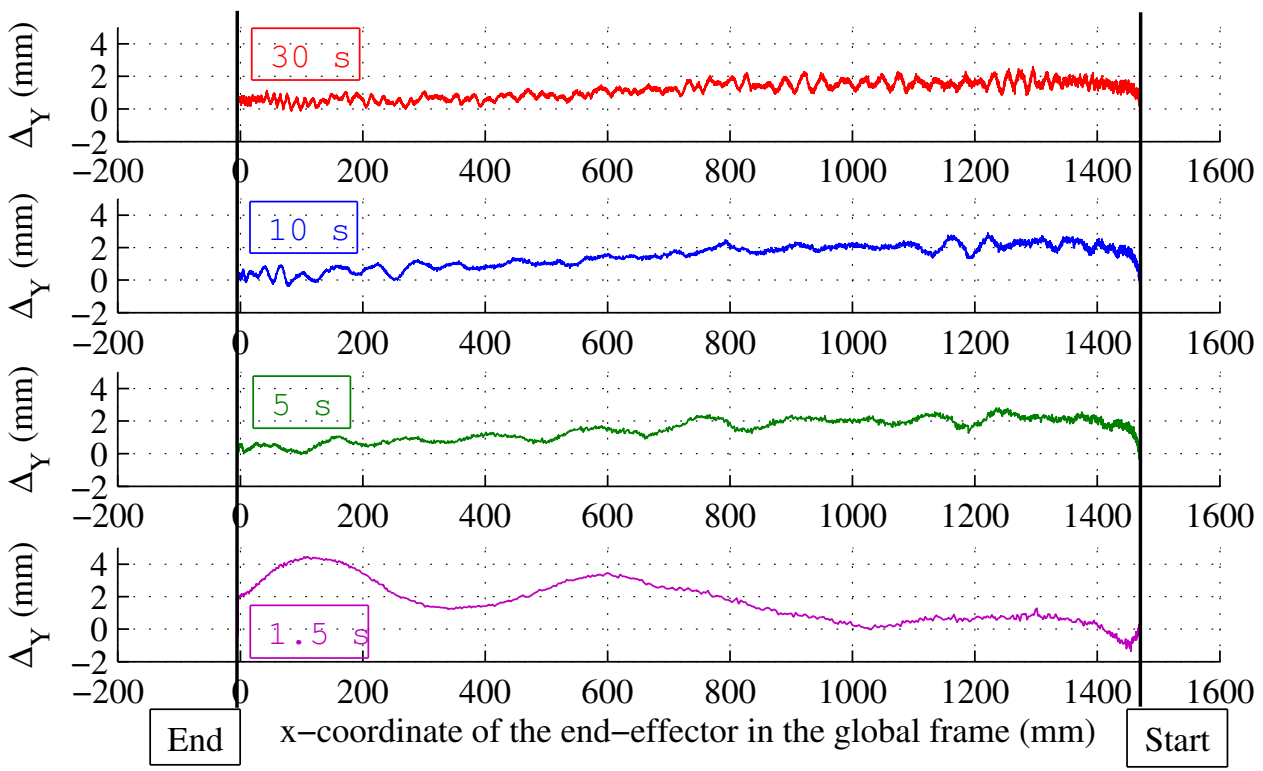

(a) Pose error of the end-effector along axis- $y_{G}$ measured in the $x_{G} O_{G} y_{G}$ plane

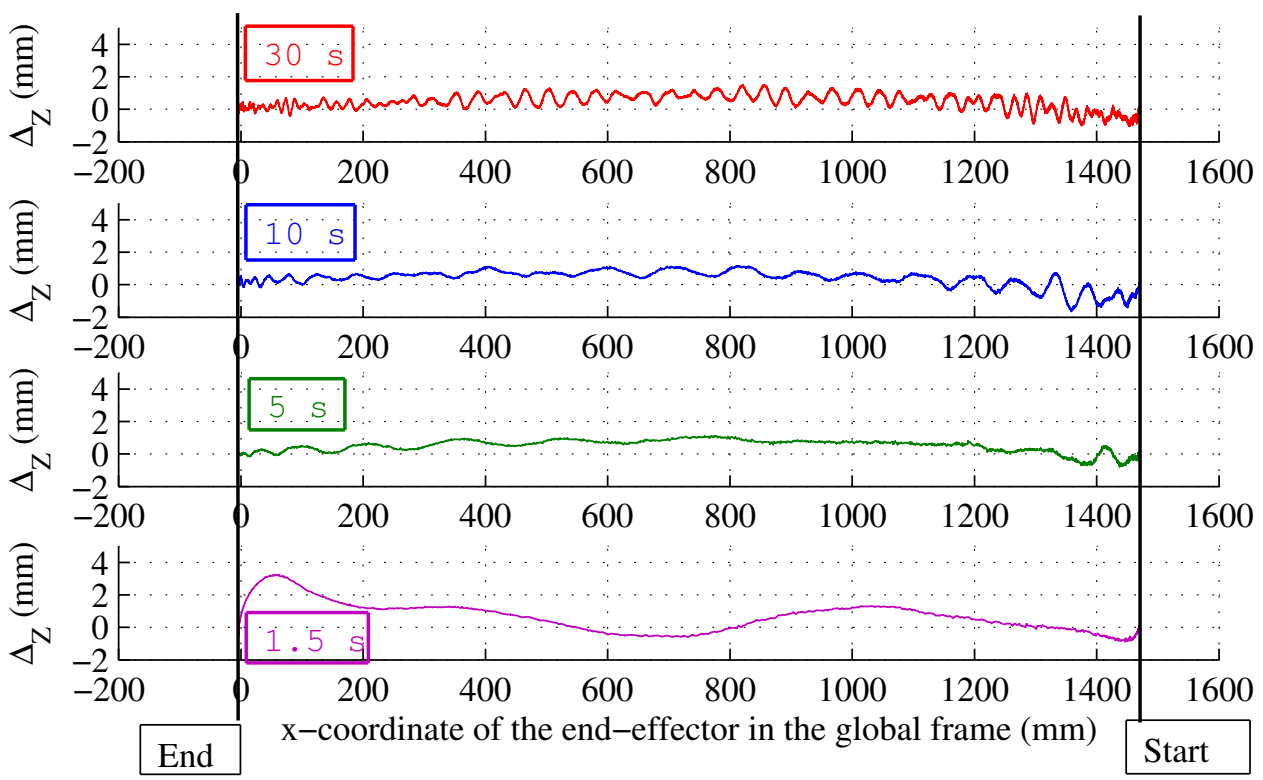

(b) Pose error of the end-effector along axis- $z_{G}$ measured in the $x_{G} O_{G} z_{G}$ plane

Fig. 4.16 Pose error of the end-effector during the trajectory from $x=1 \mathrm{~m} ; y=-2 \mathrm{~m}$; $z=0 \mathrm{~m} ; \alpha, \beta, \gamma=0^{\circ}$ to $x=-0.5 \mathrm{~m} ; y=-0.7 \mathrm{~m} ; z=-0.25 \mathrm{~m} ; \alpha, \beta, \gamma=0^{\circ}$ with different trajectory time durations

\section{Vibration of the end-effector during the trajectory}

Then, time-frequency analysis of the acceleration measurements are made to study the vibration of the end-effector during the trajectory. Figure 4.17 presents the experimental results of 


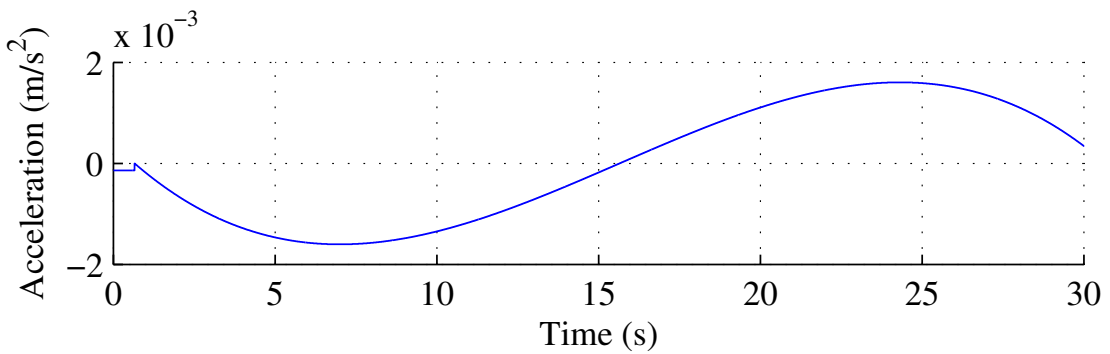

(a) Acceleration of the end-effector along axis- $z$ during the trajectory of 30 seconds (data given by the controller )

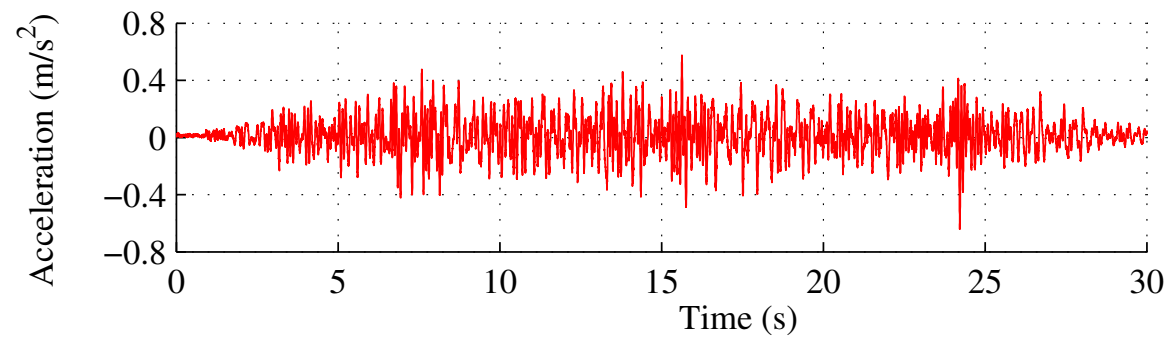

(b) Acceleration of the end-effector along axis- $z$ during the trajectory of 30 seconds (data measured by the accelerometer)

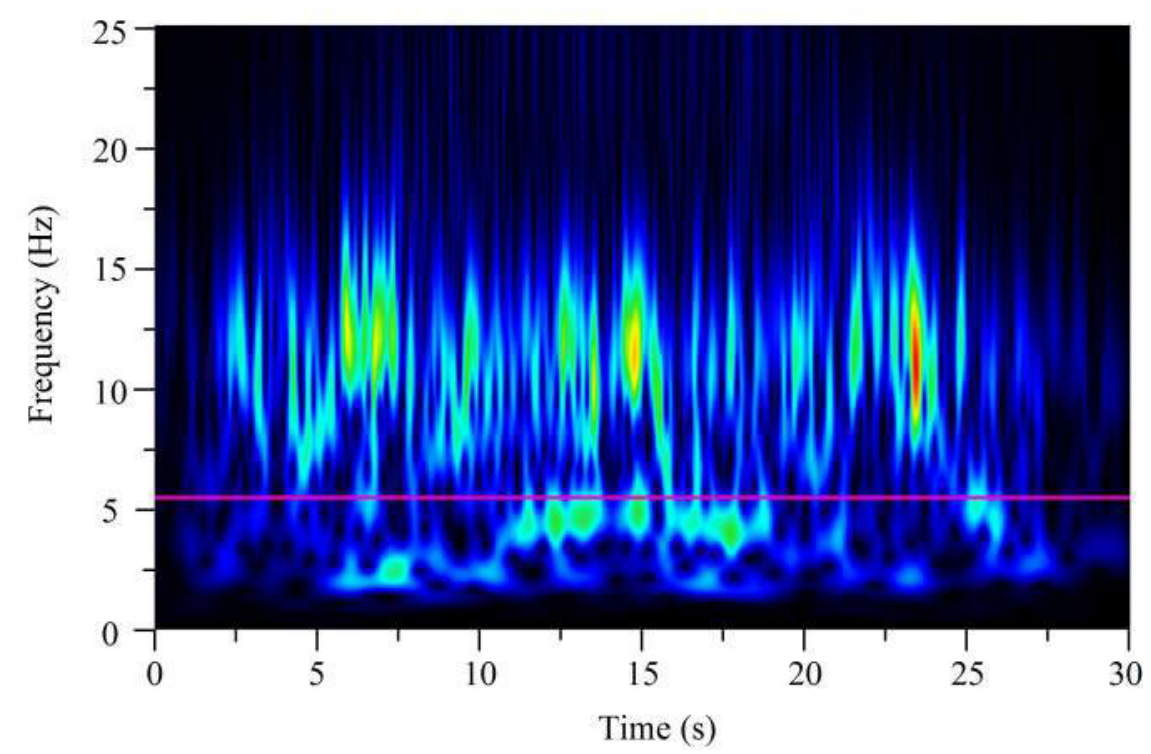

(c) Time-Frequency response of the end-effector along axis- $z$ during the trajectoryof 30 seconds

Fig. 4.17 Experimental results of the vibration analysis of the end-effector along axis- $z$ during the trajectory of 30 seconds

the vibration analysis of the end-effector along axis- $z$ during the trajectory. Acceleration of the end-effector is presented in fig. $4.17 \mathrm{~b}$, and time-frequency response of the end-effector is 
presented in fig. 4.17c. Firstly, as we can see, the peaks of the vibration amplitude appear at $7 \mathrm{~s}, 15 \mathrm{~s}, 24 \mathrm{~s}$ during the trajectory in fig. 4.17. These peaks correspond to the extreme points and the inflection point of the acceleration curve. Secondly, three groups of modes of the end-effector can be identified from fig. 4.17c: one group around $5 \mathrm{~Hz}$, another group around $7 \mathrm{~Hz}$, and the other around $12 \mathrm{~Hz}$. As we can see from fig. $4.9 \mathrm{~b}$ in section 4.3.2, these modes correspond to the end-effector modes. Therefore, the vibration of the CoGiRo along the experimental trajectory is mainly affected by the frequencies of the rigid-body modes of the end-effector, especially in low frequency.

\subsection{Summary of the chapter}

Based on the experimental and simulation results in this chapter, conclusions are made:

First of all, the effect of cable sag and external load on the static stiffness of CDPRs is validated. The experimental data are quite close to the computational ones obtained by the sagging cable model. It is shown that the sag-introduced flexibility is the main source of static robot stiffness with small external load, and the axial cable elasticity becomes the main source with big external load. The experimental results indicate the model relevance to predict the pose error due to the compliances of CDPRs. An optimization of the control scheme by using this model could be made to improve the accuracy of CDPRs.

Secondly, DSM method is validated in the dynamic modeling of cables and CDPRs and the effect of cable dynamics on the dynamic behavior of CDPRs is analyzed. Results show the validity of the proposed dynamic models. It is also indicated that the vibration of CDPRs is a strong coupling of cable and end-effector vibrations when a periodic external excitation is applied, where cable dynamics can affect robot dynamics by changing and adding new resonances. It is the case in the applications such as the cable-driven wind tunnel, the giant cable-suspended telescope and the cable-suspended machine tool, cable dynamics where a periodic excitation source is directly or indirectly applied to cables. On the free vibration and trajectory vibration analysis of CDPRs, the main contribution in these responses is principally due to the rigid-body modes of the end-effector suspended on the global cable stiffness. The energy transfer between the cables vibration and the end-effector vibration is small.

Another important result of these experiments is the importance of the trajectory profile definition. Important vibration levels appear at each extreme points and the inflection point of the acceleration. A smooth acceleration profile could reduce vibration of the end-effector. 



\section{Chapter 5}

\section{Application of the proposed methods on the force distribution of CDPRs}

\section{Contents}

5.1 Force distribution considering the effect of cable sag . . . . . . . 8 87

5.2 Determination of the lower-boundary of cable forces . . . . . . . 91

5.3 A numerical example $\ldots \ldots \ldots \ldots \ldots \ldots$

5.4 Summary of the chapter . . . . . . . . . . . . 100

One major characteristic of cables is that cables can only act in tension. If some cables loose tension, CDPRs may become unstable and even out of control. Therefore, it is important to keep all the driving cables in tension during the movement of CDPRs. To achieve this purpose, redundant actuation is usually used. This is to say, at least $n+1$ driving cables are needed to fully constrain an $n$ DOF CDPR [Kawamura+ 1993]. Due to the actuation redundancy in CDPRs, there exists infinite solutions of cable forces to balance a given wrench applied on the end-effector. As a consequence, one important design issue for redundant actuated CDPRs is the identification and the calculation of feasible cable force distribution.

The problem of the determination of force distribution is many times addressed in literature [Gosselin+ 2011; Mikelsons+ 2008; Pott+ 2009; Khosravi+ 2013; Oh+ 2005; Bruckmann+ 2006; Kawamura+ 1995; Fang+ 2004; Hassan+ 2008; Lim+ 2011]. In these studies, cables are usually assumed as ideal massless straight lines, where cable mass and/or elasticity are neglected. This assumption is not accurate, especially for CDPRs with heavy and/or long cables. The cable shape between two attachment points under the effect of gravity is not a straight line but a sagging curve. So the direction of cable forces is tangent to the sagging curve. The change of the force directions can affect the identification of 
the cable forces. Inaccurate cable forces computation can modify the equilibrium of the end-effector and thus affect the performances of CDPRs such as the positioning accuracy and the trajectory tracking due to vibration [Yuan+ 2015]. Another important issue is the determination of the lower-boundary of cable forces. In fact, in order to keep all the cables in tension, a positive lower-boundary of cable forces is used as a constraint in the identification problem of force distribution for redundant CDPRs. Small cable forces tend to cause cable sag and decrease cable stiffness [Yuan+ 2015; Arsenault 2013]. In some case, an important cable sag can even cause the end-effector to become under-constrained, and make the robot out of control [Gosselin+ 2011]. On another hand, the internal forces of all the driving cables can be increased by raising the lower-boundary used in the force distribution computation. The cable sag is decreased and cable vibration is reduced. Thus the performances of CDPRs are improved. But this can directly lead to a significant growth in motor torque and energy consumption, which enlarges both the manufacturing and the operating cost of CDPRs. The value for the lower-boundary of cable forces is chosen arbitrarily and usually the same for all driving cables [Gosselin+ 2011; Mikelsons+ 2008; Pott+ 2009]. It does not change over the entire workspace of CDPRs or along the whole trajectory of the end-effector. During a trajectory, the length of the driving cables are changing with the pose of the end-effector. If the lower-boundary of cable forces is fixed, some driving cables may have more than enough tension to maintain the cable sag low, which is a waste of motor torque and energy. While some other driving cables may not have adequate tensions to reach the required stiffness, which decreases the performances of cables and CDPRs. Thus the drawback of fixed lowerboundary emerges. Different cables at different poses of the end-effector have different requirements of cable forces to achieve the required performances. Fixed lower-boundary method cannot consider or reflect these requirements.

In this chapter, a new force distribution method with pose-dependent force boundaries for redundant actuated CDPRs is proposed. The main contributions of this chapter are the following:

- An efficient force distribution method taking into account the effect of cable sag is presented. An optimization method with a user-defined cost function is defined to solve the force distribution problem and to obtain accurate cable forces.

- An original method is proposed to determine the lower force boundary used as constraint in the force distribution. The lower-boundary of cable forces can be calculated according to the cable's fundamental frequency, and thus is directly associated with the CDPR performances. 
- The lower-boundary of each driving cable is calculated for every pose of the endeffector along a trajectory. The method can give out much more suitable force boundaries for every cable and thus guarantee the cable performances according to the design requirement while not stretch the cables too much.

This chapter is organized as follows. Kinematics modeling and force distribution are presented in section 5.1. Then a new method on the determination of the lower-boundary of cable forces is proposed in section 5.2, including the fixed boundary and the pose-dependent boundary. Simulations on a 6-DOF CDPR driven by 8 cables are presented as an example in section 5.3. Finally, summary is made in section 5.4.

\subsection{Force distribution considering the effect of cable sag}

\subsubsection{CDPR description}

The kinematic model and force distribution are independent of each other if the effect of cable weight on the cable shape is neglected [Gosselin+ 2011; Mikelsons+ 2008; Pott+ 2009]. Considering cable sag, the kinematics of CDPRs is coupled with the determination of cable forces. An optimization method with a user-defined cost function is proposed in this section to solve the coupling problem and identify accurate cable force distribution.

A general CDPR is presented schematically in fig. 5.1, where:

- $A_{i}$ is the attachment point in the end-effector; $B_{i}$ is the attachment point in the fixed base; and $L_{i}$ is the $i^{\text {th }}$ driving cable connecting these two points.

- $\mathfrak{R}_{G}\left(O_{G}, x_{G}, y_{G}, z_{G}\right)$ is the fixed global frame; $\mathfrak{R}_{e}\left(O_{e}, x_{e}, y_{e}, z_{e}\right)$ is the local frame attached to the end-effector; $\mathfrak{R}_{c i}\left(O_{c i}, x_{c i}, y_{c i}, z_{c i}\right)$ is the local cable frame, where axis $z_{c i}$ is parallel to the direction of gravity, and axis $y_{c i}$ is perpendicular to the cable plane.

- $\alpha_{i}$ is the inclination angle; $l_{c i}$ is the chord length; $d_{i}$ is the sag perpendicular to the chord; $\tau_{i}$ is the cable tension at the section where cable is parallel to its chord.

- $f_{A i}$ is the cable force at point $A_{i} . f_{A i x}$ and $f_{A i z}$ are the components of $f_{A i}$ along $x_{c i}$ and $z_{c i}$ axis respectively.

As shown in fig. 5.1, the cable profile between two attachment points $A_{i}$ and $B_{i}$ is not a straight line but a sagging curve under the effect of gravity. According to [Irvine 1992], the profile of the sagging curve can be described as a function of the cable force and cable length. 


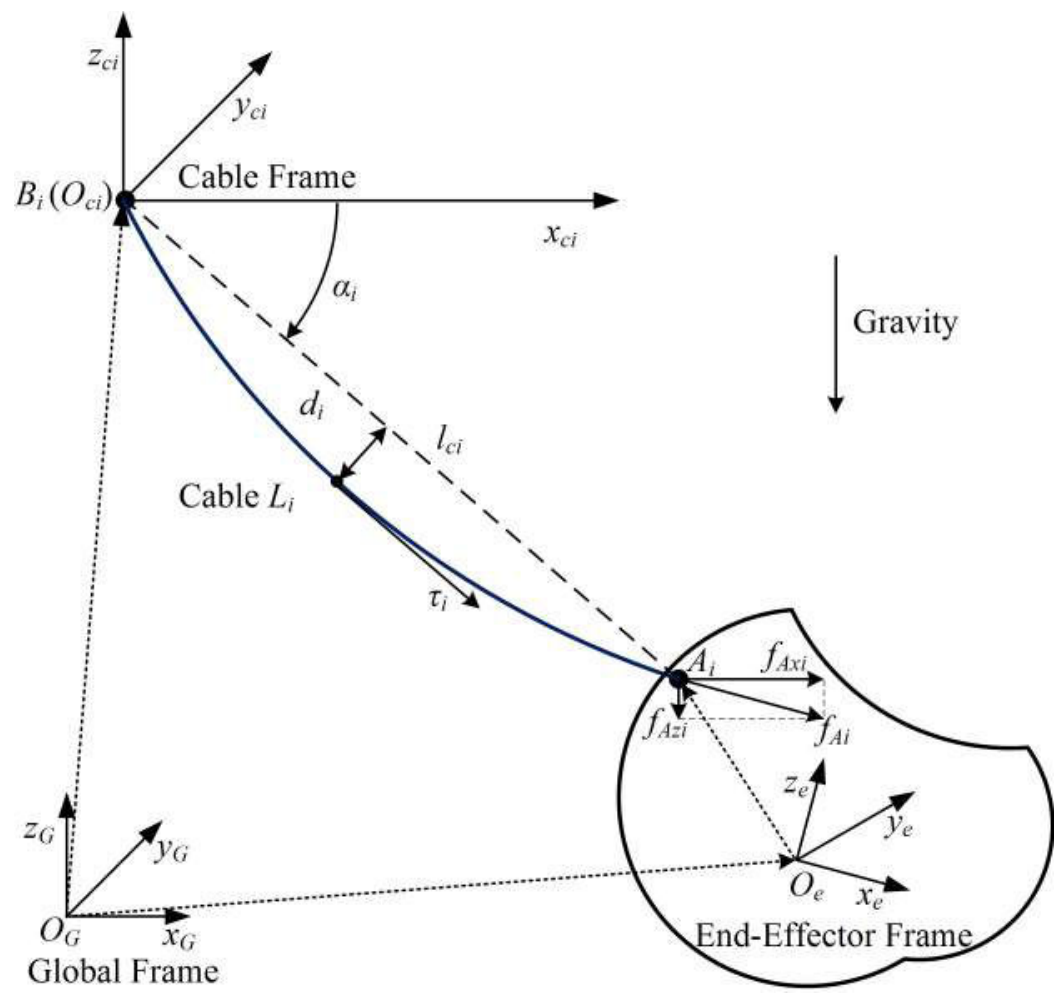

Fig. 5.1 Kinematic model of a general CDPR considering cable sag

Moreover, the direction of the cable force at the attachment point $A_{i}$ is not along the straight line between $A_{i}$ and $B_{i}$, but tangential to the sagging curve at $A_{i}$. Thus the direction of the cable force depends on the cable profile. Therefore, the cable force are coupled with the cable length. It means that the kinematics and the force distribution of CDPRs are coupled and they should be solved together.

\subsubsection{Kinematic modeling}

The objective of the kinematics of CDPRs is to calculate the unstrained cable length for a given pose of the end-effector (position and orientation). The objective of the force distribution is to compute the cable forces for a given pose of the end-effector to balance a given external wrench (force and moment). Considering the coupling of the kinematics and the force distribution, the problem can be formulated as: for a given external wrench and a given end-effector pose, find the unstrained cable lengths and the proper cable forces which satisfy the following constraints.

1) Equality constraints describing the relationship between cable forces and unstrained cable 
lengths [Irvine 1992]:

$$
\begin{aligned}
& { }^{c i}{\overrightarrow{O_{c i} A_{i}}}^{(x)}=\frac{f_{A x i} l_{u s i}}{E A}+\frac{\left|f_{A x i}\right|}{\rho g}\left[\sinh ^{-1}\left(\frac{f_{A z i}}{f_{A x i}}\right)-\sinh ^{-1}\left(\frac{f_{A z i}-\rho g l_{u s i}}{f_{A x i}}\right)\right], \\
& { }^{c i}{\overrightarrow{O_{c i} A_{i}}}^{(y)}=0 \\
& c i{\overrightarrow{O_{c i} A_{i}}}^{(z)}=\frac{f_{A z i} l_{u s i}}{E A}-\frac{\rho g l_{u s i}^{2}}{2 E A}+\frac{1}{\rho g}\left[\sqrt{f_{A x i}^{2}+f_{A z i}^{2}}-\sqrt{f_{A x i}^{2}+\left(f_{A z i}-\rho g l_{u s i}\right)^{2}}\right],
\end{aligned}
$$

where ${ }^{c i}{\overrightarrow{O_{c i} A_{i}}}^{(x)},{ }^{c i}{\overrightarrow{O_{c i} A_{i}}}^{(y)}$ and ${ }^{c i}{\overrightarrow{O_{c i} A_{i}}}^{(z)}$ are respectively the $x, y$ and $z$ coordinates of $A_{i}$ expressed in the cable frame $\mathfrak{R}_{c i} ; l_{u s i}$ is the unstrained cable length of $L_{i} ; E$ is the Young's modulus; $A$ is unstrained cross-sectional area; $\rho$ is the cable mass per unit length; $g$ is the gravitational acceleration.

2) Equality constraints on the static equilibrium of the end-effector:

$$
\begin{gathered}
\sum_{i=1}^{m}{ }^{G} \mathbf{f}_{A i}+{ }^{G} \mathbf{f}_{e x}=\mathbf{0}, \\
\sum_{i=1}^{m}\left({ }^{G} \overrightarrow{O_{e} A_{i}} \times{ }^{G} \mathbf{f}_{A i}\right)+{ }^{G} \mathbf{m}_{e x}=\mathbf{0}, \\
{ }^{G} \mathbf{f}_{A i}={ }^{G} \mathbf{T}_{c i}{ }^{c i} \mathbf{f}_{A i}={ }^{G} \mathbf{T}_{c i}\left[-f_{A x i} 0-f_{A z i}\right]^{T},
\end{gathered}
$$

where ${ }^{G} \mathbf{f}_{e x}$ and ${ }^{G} \mathbf{m}_{e x}$ are respectively the external force and moment vectors expressed in global frame; ${ }^{G} \mathbf{f}_{A i}$ and ${ }^{c i} \mathbf{f}_{A i}$ are the force vectors at point $A_{i}$ expressed in the global frame $\mathfrak{R}_{G}$ and the cable frame $\mathfrak{R}_{c i}$, respectively; ${ }^{G} \mathbf{T}_{c i}$ is the rotation matrix that maps the coordinates in $\mathfrak{R}_{c i}$ to their corresponding coordinates in $\Re_{G}$.

3) Equality constraints representing the geometric relationship:

$$
\begin{aligned}
{ }^{c i} \overrightarrow{O_{c i} A_{i}} & ={ }^{G} \mathbf{T}_{c i}^{-1}{ }^{G} \overrightarrow{O_{c i} A_{i}}={ }^{G} \mathbf{T}_{c i}^{-1}\left({ }^{G} \overrightarrow{O_{G} O_{e}}+{ }^{G} \overrightarrow{O_{e} A_{i}}-{ }^{G} \overrightarrow{O_{G} O_{c i}}\right) \\
& ={ }^{G} \mathbf{T}_{c i}^{-1}\left({ }^{G} \overrightarrow{O_{G} O_{e}}+{ }^{G} \mathbf{T}_{e} e \overrightarrow{O_{e} A_{i}}-{ }^{G} \overrightarrow{O_{G} O_{c i}}\right)
\end{aligned}
$$

where $\overrightarrow{O_{G} O_{e}}$ and $G \overrightarrow{O_{G} O_{c i}}$ are respectively the coordinates of points $O_{e}$ and $O_{c i}$ expressed in the global frame $\mathfrak{R}_{G} ; \vec{O}_{e} A_{i}$ is the coordinate of point $A_{i}$ expressed in the end-effector frame $\mathfrak{R}_{e} ;{ }^{G} \mathbf{T}_{e}$ is the rotation matrix that transfers coordinates expressed in $\Re_{e}$ to their corresponding coordinates expressed in $\Re_{G}$. 
4) Inequality constraints about the boundaries of cable forces and lengths:

$$
\begin{array}{r}
0<l_{u s i} \leq l_{\text {imax }} \\
f_{\text {imin }} \leq\left|\mathbf{f}_{A i}\right|=\sqrt{f_{A x i}^{2}+f_{A z i}^{2}} \leq f_{\text {imax }}
\end{array}
$$

where $l_{i \max }$ is the maximum cable length; $f_{i \min }$ and $f_{i \max }$ are the lower-boundary and higher-boundary of cable force.

\subsubsection{Force distribution}

For an $n$-DOF CDPR driven by $m$ cables, there are $2 m$ equations from the sagging cable model and $n$ equations from the static equilibrium of the end-effector. While there are $3 m$ unknowns: $m$ unknown cable lengths $l_{u s i}$ and $2 m$ unknown cable forces $f_{A x i}$ and $f_{A z i}$ $(i=1,2 \cdots m)$. Because of the CDPRs discussed in this chapter are redundant actuated, the number of driving cables is more than the degree of freedom of the end-effector $(m>n)$. Thus there are more unknowns than constraints $(3 m>2 m+n)$. There may exist infinite solutions for the force distribution of redundant actuated CDPRs. Therefore, optimization methods with a user-defined cost function are usually employed to obtain an unique solution. Mathematically, the problem solution can be formulated as following:

Force distribution of redundant actuated CDPRs
Objective: $\min f($ variables $)$
Variables: $f_{A x i}, f_{A z i}, l_{u s i}$ and $i=1,2, \cdots m$
Equality constraints: $(5.1) \sim(5.7)$
Inequality constraints: $(5.8)$ and (5.9)

where $f$ (variables) is the user-defined cost function. In this chapter, the Euclidean norm of the force vector $\sqrt{\sum_{i=1}^{m}\left(f_{A x i}^{2}+f_{A z i}^{2}\right)}$ is defined as the cost function, because minimization of the cable forces is important to minimize the size of the actuators during design and save energy consumption during operation, and thus to reduce the manufacturing and operational costs of CDPRs. It should be noted that the lower-boundary in eq. (5.9) is always an arbitrary fixed value in literature [Gosselin+ 2011; Mikelsons+ 2008; Pott+ 2009; Khosravi+ 2013; Oh+ 2005; Bruckmann+ 2006; Kawamura+ 1995; Hassan+ 2008; Lim+ 2011]. In this chapter, we propose a method on the determination of the lower-boundary. This method will be detailed in the following section.

Another important issue of the above optimization algorithm is the initial iteration point. Because a good initial estimate is vital for the convergence and efficiency of the algorithm. 
Assuming that cables are ideal straight lines without mass and elasticity, thus the kinematics and the force distribution of CDPRs are uncoupled. The ideal cable lengths can be calculated through the inverse kinematics $\left|\overrightarrow{A_{i} B_{i}}\right|=\left|\vec{O}_{G} B_{i}-G \overrightarrow{O_{G} O_{e}}-{ }^{G} \mathbf{T}_{e} e^{e} \overrightarrow{O_{e} A_{i}}\right|$. While the ideal cable forces can be obtained by the following optimization method:

Initial iteration of cable forces
Objective: $\min _{f_{i}} \sqrt{\left(\sum_{i=1}^{m} f_{i}^{2}\right)}$
Constraints: $\mathbf{J}^{T} \mathbf{f}_{\text {ideal }}+\mathbf{w}_{e x}=\mathbf{0}$
Boundaries: $f_{i \min } \leq f_{i} \leq f_{\text {imax }}$

where $\mathbf{J}^{T}$ is the transposition of Jacobian matrix, $\mathbf{f}_{\text {ideal }}=\left[f_{1} f_{2} \cdots f_{m}\right]^{T}$ is the ideal cable force vector, and $\mathbf{w}_{e x}$ is the column vector that represents the external wrench consisting of the forces ${ }^{G} \mathbf{f}_{e x}$ and the moments ${ }^{G} \mathbf{m}_{e x}$. Then the ideal cable lengths and forces can be used as the initial iteration.

\subsection{Determination of the lower-boundary of cable forces}

\subsubsection{Lower force boundary definition}

As explained before, the determination of a proper lower-boundary of cable forces is an important issue of the force distribution of CDPRs. In our previous research [Yuan+ 2014], it is shown that cable dynamics can effect the vibration of CDPRs by changing the value of robot natural frequencies and/or add new resonances. The fundamental frequency of cables is a good index to evaluate cable stiffness. Cable vibration becomes the key factor for some applications, such as the wind-induced vibration of the wind tunnel [Bruckmann+2010] and the large radio telescope [Du+ 2012]. Therefore, studying and controlling cable vibration are significant to improve the performances of CDPRs. This section will present the relationship between cable forces and cable's fundamental frequency. The lower-boundary of cable forces will be determinated according to this relation.

Cable dynamics has been well studied in civil engineering [Irvine 1978; Starossek 1991b]. According to [Irvine 1978], the relationship between cable force and the cable's fundamental frequency can be expressed as:

$$
\Omega=\pi \sqrt{\frac{\tau}{\rho g l_{c}}}
$$

where $\Omega$ is the cable's fundamental frequency; $\tau$ is the cable tension at the section where cable 
is parallel to its chord (fig. 5.1); $\rho$ is the cable mass per unit length; $l_{c}$ is the chord length. For small cable sag, the chord length $l_{c}$ and cable tension $\tau$ are approximate to the unstrained cable length $l_{u s}$ and cable force $f_{A}$ respectively. Equation (5.10) can be reformulated as:

$$
\Omega \simeq \pi \sqrt{\frac{f_{A}}{\rho g l_{u s}}}=\pi \sqrt{\frac{f_{A}}{W_{c}}},
$$

where $W_{c}=\rho g l_{u s}$ representing the cable weight. Thus the relationship of the cable fundamental frequency $\Omega$, the cable weight $W_{c}$ and the cable force $f_{A}$ can be described by eq. (5.11).

As explained before, the dynamic behaviors of cables and CDPRs are associated with the cable's fundamental frequency $\Omega$. Increasing the cable length can decrease the cable's fundamental frequency, even if the cable force remains the same. It means that shorter cable need smaller force to maintain the cable's fundamental frequency (ie the cable stiffness) at a certain value. Enhancing $\Omega$ can increase system natural frequencies and reduce the vibration of cables and the end-effector. To guarantee the dynamic performances, a minimum value of $\Omega$ can be set as:

$$
\Omega \geq b,
$$

where $b$ is a constant value presenting the cable's fundamental frequency. Substituting eq. (5.11) into eq. (5.12) yields:

$$
f_{A} \geq \frac{b^{2}}{\pi^{2}} W_{c}
$$

\subsubsection{Pose-dependency}

If we consider the movement of the end-effector in a trajectory, the weights $W_{c}$ of different driving cables at a certain pose of the end-effector are usually not the same, and the weight of a certain driving cable is also changing with the pose of the end-effector during the trajectory. Therefore, in eq. (5.13), $W_{c}$ is a function of the end-effector pose $j$ and the cable serial number $i$, so is the cable force $f_{A}$. Equation (5.13) can be reformulated as:

$$
f_{A(i, j)} \geq \frac{b^{2}}{\pi^{2}} W_{c(i, j)} .
$$




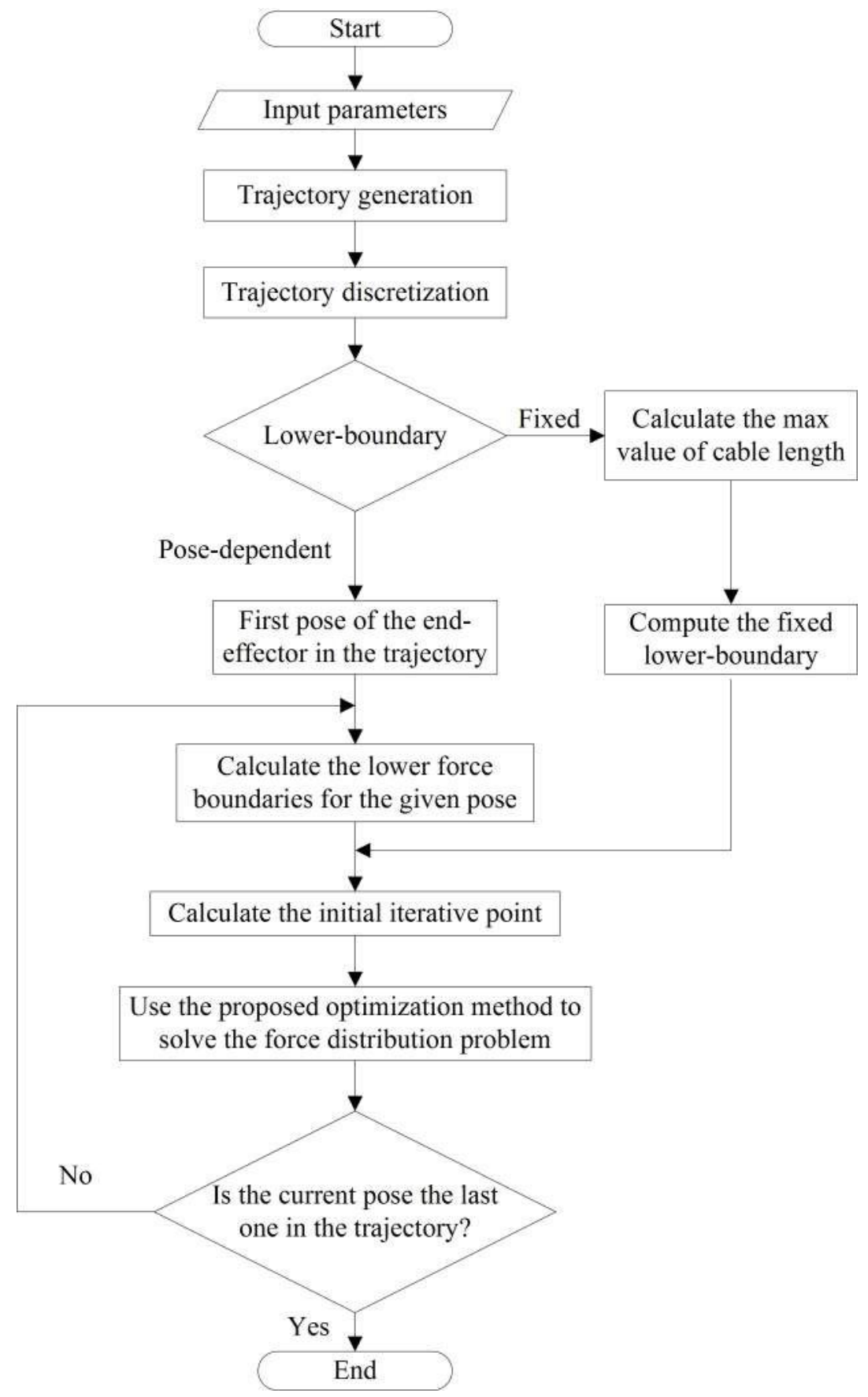

Fig. 5.2 Flow chart of the force distribution of CDPRs

Consequently, the pose-dependent lower-boundary of the $i^{t h}$ cable at the $j^{\text {th }}$ pose of the end-effector in the trajectory $f_{\mathrm{lb}(i, j)}$ can be written as:

$$
f_{\mathrm{lb}(i, j)}=\frac{b^{2}}{\pi^{2}} W_{c(i, j)}
$$


With this pose-dependent lower-boundary, the cable's fundamental frequency is assuringly higher than the constant value $b$ during all the trajectory.

In some cases, a fixed lower-boundary for all the cables is required for the controller. To guarantee that the fundamental frequencies of all cables meet the requirement, the fixed lower-boundary can be defined as:

$$
f_{\mathrm{lb}}=\frac{b^{2}}{\pi^{2}} \max _{i, j}\left(W_{c(i, j)}\right)
$$

where $\max _{i, j}\left(W_{c(i, j)}\right)$ presents the maximum cable weight among all the driving cables for every pose of the end-effector in the trajectory.

Figure 5.2 gives the calculation process to solve the force distribution problem of CDPRs. Firstly, parameters about the cables, the end-effector, and the CDPR are defined. Secondly, the trajectory of the end-effector is generated, and discretization is made in order to get an array consisting of all the discrete poses of the end-effector along the trajectory. The step length of the discretization can be determinated according to the accuracy requirement. Thirdly, the lower-boundary of cable forces is determinated. If fixed lower-boundary is chosen in the algorithm, the max value of cable length among all the cables and poses in the trajectory is found out. The fixed lower-boundary is then computed according to eq. (5.16). If pose-dependent lower-boundary is chosen in the calculation, the lower-boundaries of all the driven cables at a given pose of the end-effector in the trajectory can be computed according to eq. (5.15). Fourthly, the optimization method proposed in section 5.1 is used at each pose of the end-effector along the trajectory, and the optimized cable forces are calculated.

\subsection{A numerical example}

A 6-DOF CDPR driven by 8 cables is presented in this section as an example to illustrate how to determinate the lower-boundary of cable forces and to calculate the force distribution with the proposed methods. Two kinds of force boundary presented in section 5.2, the fixed lower-boundary and the pose-dependent lower-boundary, are calculated in the force distribution method proposed in section 5.1.

\subsubsection{Description of the studied mechanism}

The 6-DOF CDPR is presented schematically in fig. 5.3. There are 8 attachment points on the 4 fixed vertical poles and 8 corresponding attachment points on the vertexes of the cube that 


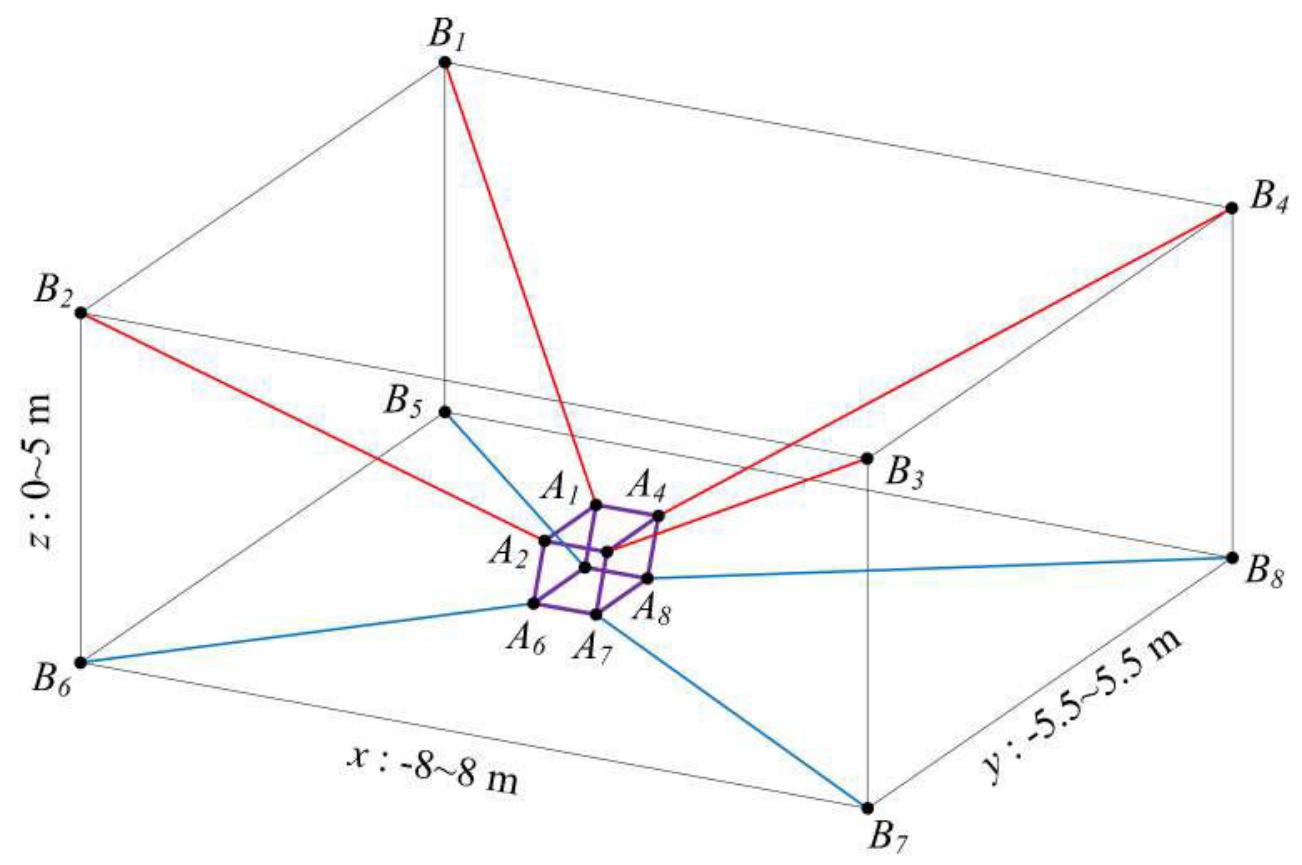

Fig. 5.3 Configuration of the 6 DOF CDPR driven by 8 cables

serves as an end-effector. 8 driving cables are used to connect the corresponding attachment points. Parameters of the cables and the end-effector are listed in table 5.1. The configuration parameters of the CDPR are given in table 5.2.

Table 5.1 Parameters of the end-effector and the driving cables

\begin{tabular}{|l|l|}
\hline Young's modulus $E$ & $17.7 \times 10^{9} \mathrm{GPa}$ \\
\hline Unstrained cross-sectional area $A$ & $13.1 \mathrm{~mm}^{2}$ \\
\hline Cable mass per meter $\rho$ & $0.12 \mathrm{~kg} / \mathrm{m}$ \\
\hline Mass of the end-effector $m$ & $70 \mathrm{~kg}$ \\
\hline Inertia matrix of the end-effector & $\operatorname{diag}(8.313 .213 .2) \mathrm{kg} \cdot \mathrm{m}^{2}$ \\
\hline
\end{tabular}


Table 5.2 Coordinates of the attachments $B_{i}$ in the global frame and $A_{i}$ in the local end-effector frame

\begin{tabular}{|c|c|c|c||c|c|c|c|}
\hline$(\mathrm{m})$ & $x$ & $y$ & $z$ & & $x$ & $y$ & $z$ \\
\hline \hline$A_{1}$ & -0.48 & 0.32 & 0.32 & $B_{1}$ & -8 & 5.5 & 5 \\
\hline$A_{2}$ & -0.48 & -0.32 & 0.32 & $B_{2}$ & -8 & -5.5 & 5 \\
\hline$A_{3}$ & 0.48 & -0.32 & 0.32 & $B_{3}$ & 8 & -5.5 & 5 \\
\hline$A_{4}$ & 0.48 & 0.32 & 0.32 & $B_{4}$ & 8 & 5.5 & 5 \\
\hline$A_{5}$ & -0.48 & 0.32 & -0.32 & $B_{5}$ & -8 & 5.5 & 0 \\
\hline$A_{6}$ & -0.48 & -0.32 & -0.32 & $B_{6}$ & -8 & -5.5 & 0 \\
\hline$A_{7}$ & 0.48 & -0.32 & -0.32 & $B_{7}$ & 8 & -5.5 & 0 \\
\hline$A_{8}$ & 0.48 & 0.32 & -0.32 & $B_{8}$ & 8 & 5.5 & 0 \\
\hline
\end{tabular}

\subsubsection{Simulation results}

A trajectory in Cartesian space is chosen for the simulation of the 6-DOF CDPR. It is a circular helix that can be mathematically described as:

$$
\begin{aligned}
& \theta=24 \pi\left(\frac{t}{T}\right)^{5}-60 \pi\left(\frac{t}{T}\right)^{4}+40 \pi\left(\frac{t}{T}\right)^{3}, \\
& x=r \cos \theta \\
& y=r \sin \theta \\
& z=18\left(\frac{t}{T}\right)^{5}-45\left(\frac{t}{T}\right)^{4}+30\left(\frac{t}{T}\right)^{3}+1
\end{aligned}
$$

where $T$ is the total time of the trajectory, and $r$ is the radius of the circular helix. For the simulation in this chapter, $T=30 \mathrm{~s}$ and $r=3 \mathrm{~m}$. The maximum velocity and acceleration of the end-effector are $2.4 \mathrm{~m} / \mathrm{s}$ and $1.9 \mathrm{~m} / \mathrm{s}^{2}$, respectively. It should be noted:

- This trajectory is specially designed so that the velocity and acceleration of the endeffector at the start and end point of the trajectory are all zero, and the velocity and acceleration curves along the trajectory are continuous.

- The end-effector has only translational motion during the trajectory, the rotation angles of the end-effector around axis- $x, y, z$ are all constant and equal to zero.

- Dynamic forces due to the end-effector inertia are taken into account, and there is no external wrench applied on the end-effector except gravity.

- Trajectory discretization is achieved by discretizing the time of the trajectory $T$, where the step length is 0.01 second. 
The force distribution of the studied CDPR is computed using the method proposed in section 5.1. Two kinds of lower-boundary of cable forces are taken into consideration. One is the fixed lower-boundary presented by eq. (5.16), and the other is the pose-dependent lower-boundary presented by eq. (5.15). The parameter $b$ in eqs. (5.15) and (5.16) is set to be $4 \pi \mathrm{rad} / \mathrm{s}$ according to the experimental results in our previous research [Yuan+ 2015].

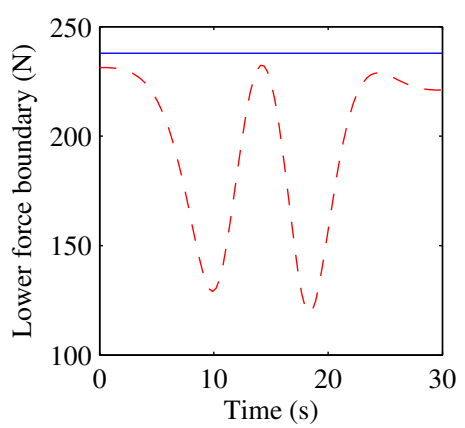

(a) $1^{\text {st }}$ cable

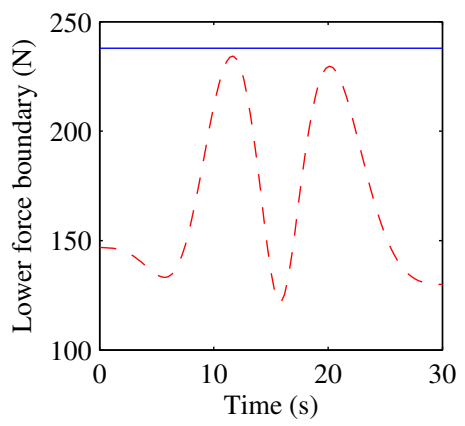

(d) $4^{\text {th }}$ cable

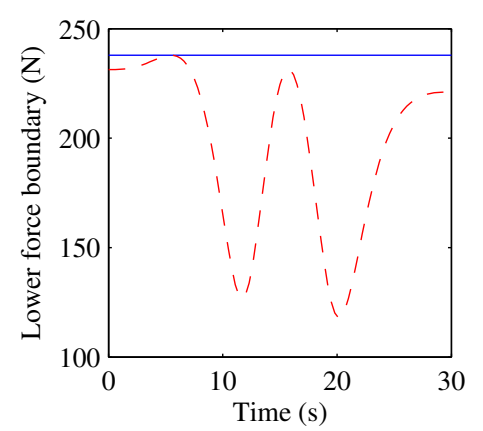

(b) $2^{\text {nd }}$ cable

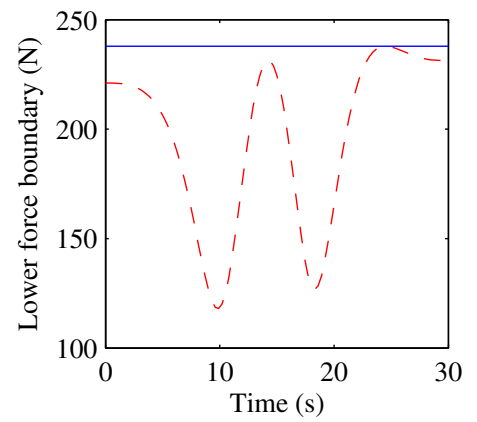

(e) $5^{\text {th }}$ cable

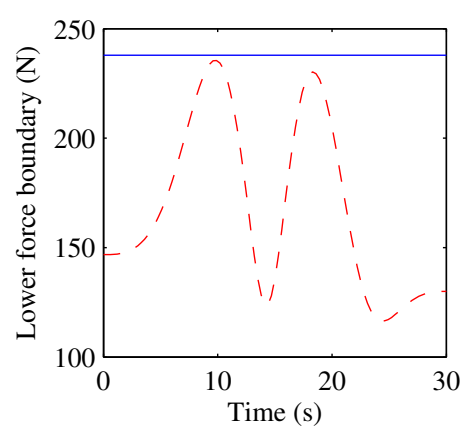

(c) $3^{\text {rd }}$ cable

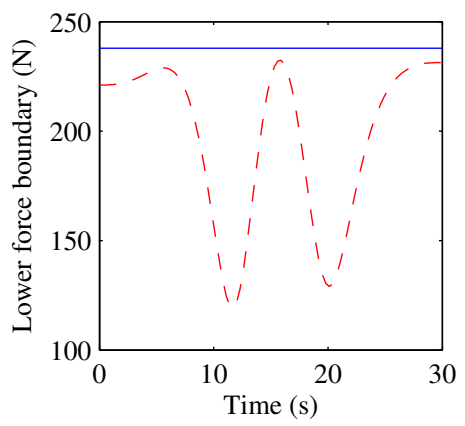

(f) $6^{\text {th }}$ cable

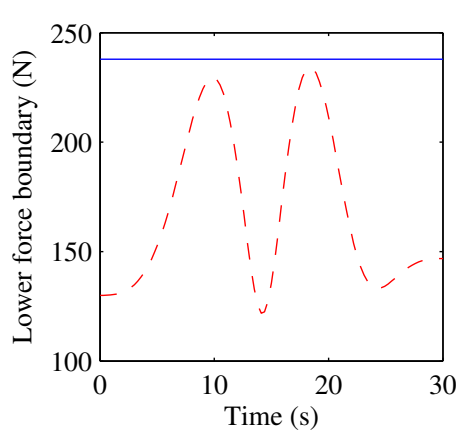

(g) $7^{\text {th }}$ cable

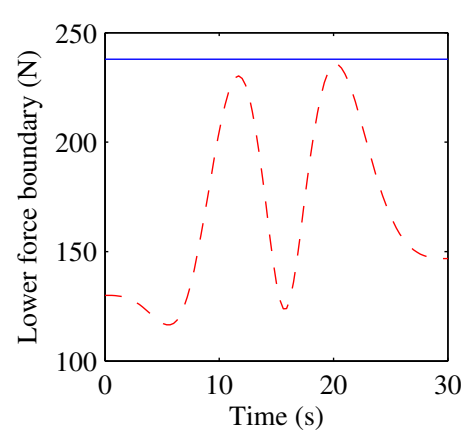

(h) $8^{\text {th }}$ cable

Fig. 5.4 The variation of the lower-boundary along the trajectory: the blue solid lines represent the results of the fixed lower-boundary; the red dash lines represent the results of the pose-dependent lower-boundary 


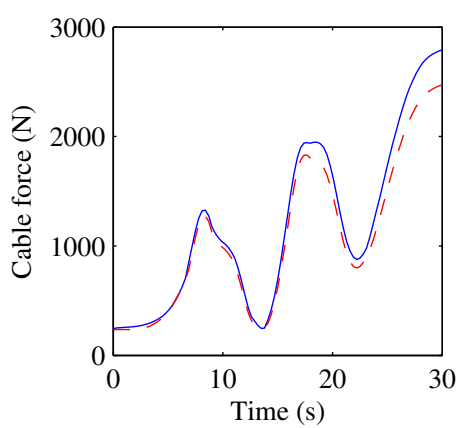

(a) $1^{\text {st }}$ cable

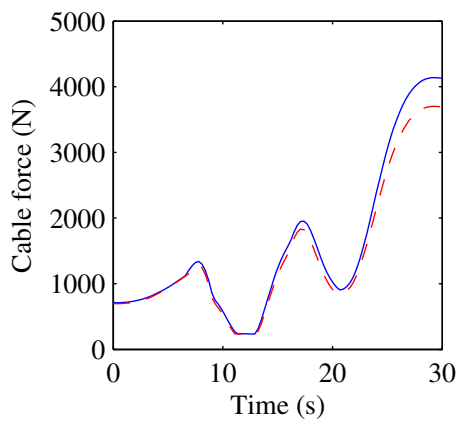

(d) $4^{\text {th }}$ cable

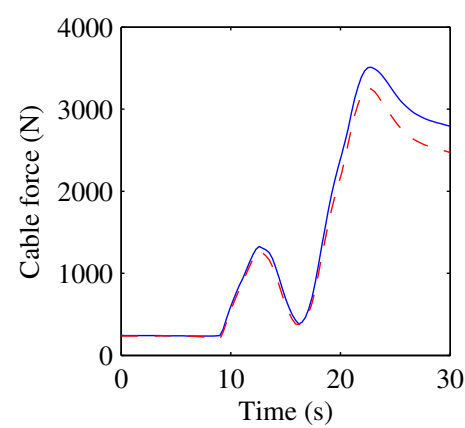

(b) $2^{\text {nd }}$ cable

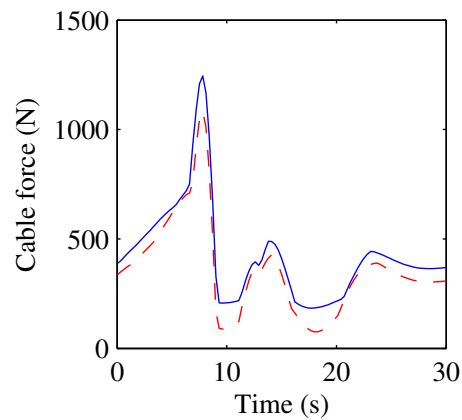

(e) $5^{\text {th }}$ cable

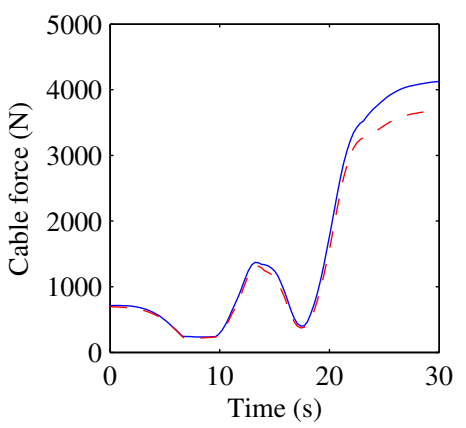

(c) $3^{\text {rd }}$ cable

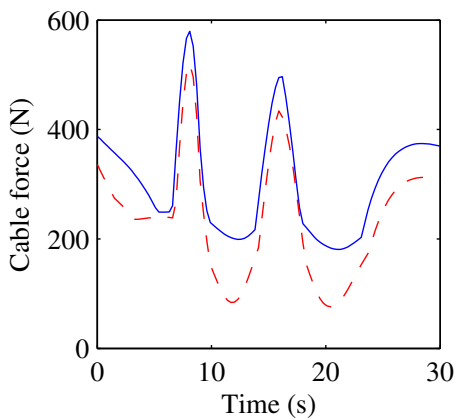

(f) $6^{\text {th }}$ cable

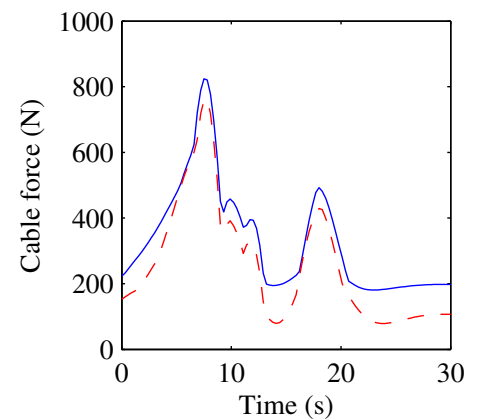

(g) $7^{\text {th }}$ cable

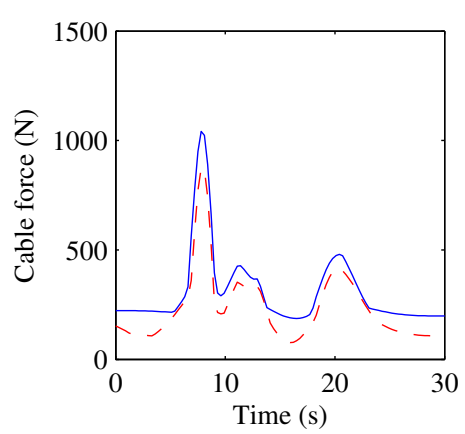

(h) $8^{\text {th }}$ cable

Fig. 5.5 The variation of the cable force along the trajectory: the blue solid lines represent the results of the fixed lower-boundary; the red dash lines represent the results of the posedependent lower-boundary

Simulation results are shown in figs. 5.4 to 5.6. Figure 5.4 and fig. 5.5 represent the variation of the lower-boundaries and the cable forces along the given trajectory, respectively. Besides force distributions, energy consumption of the CDPR is shown in fig. 5.6, where the vertical axis represents the summation of the energy consumed from $0 \mathrm{~s}$ to $t \mathrm{~s}(0 \leq t \leq 30 \mathrm{~s})$. In these figures, the blue solid lines represent the results of the fixed lower-boundary and the red dash lines represent the results of the pose-dependent lower-boundary. 


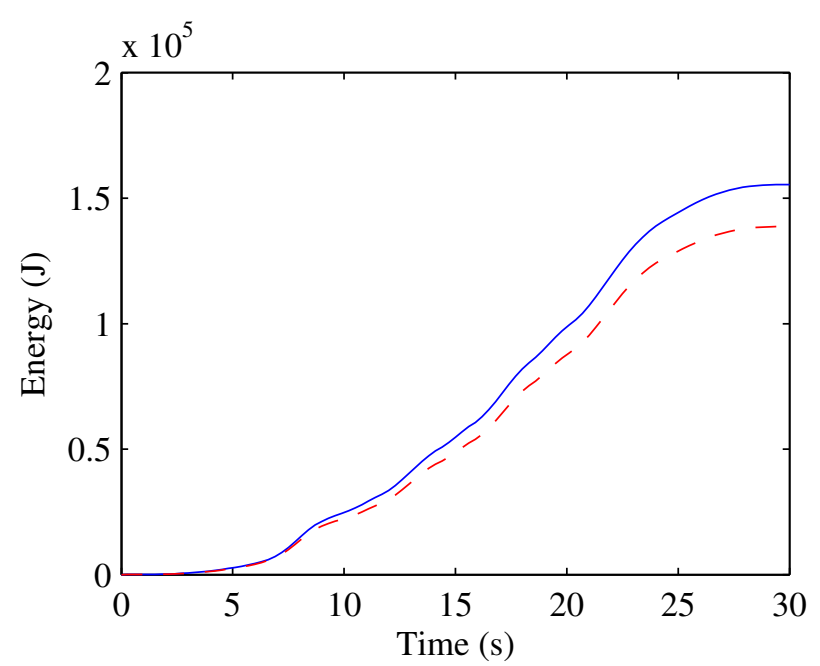

Fig. 5.6 The energy consumption of the CDPR along the trajectory: the blue solid lines represent the results of the fixed lower-boundary; the red dash lines represent the results of the pose-dependent lower-boundary

\subsubsection{Analysis and discussion}

As explained in section 5.2, the pose-dependent boundaries are function of both the endeffector pose $j$ and the cable serial number $i$, which can be calculated by eq. (5.15). However, the fixed boundary defined by eq. (5.16) is the maximum value of the force boundary among all the driving cables and all the poses of the end-effector in the trajectory. In this example, the fixed boundary can be defined by the $2^{\text {nd }}$ cable at $t=5.4 \mathrm{~s}$, or by the $5^{\text {th }}$ cable at $t=24.6 \mathrm{~s}$. Therefore, in fig. 5.4, obvious variations can be found for the pose-dependent lower-boundary and a constant value appears for the fixed lower-boundary.

As we can see from fig. 5.5, the cable forces obtained by the pose-dependent lowerboundary method are all smaller than those obtained by fixed lower-boundary method. In addition, the maximum force of each cable is identified and listed in table 5.3. The maximum cable force is directly associated with the size and torque of motors, which is an important parameter for the design of CDPRs. Compared to fixed lower-boundary method, posedependent lower-boundary method can obviously reduce the maximum cable force of each driving cable. For the $8^{\text {th }}$ cable, this reduction even reaches $19 \%$.

Besides cable forces, energy consumption of the CDPR is also analyzed through fig. 5.6. As we can see, the energy consumption of the motor calculated by the pose-dependent lower-boundary method is lower than that by the fixed lower-boundary method. For the given trajectory, the CDPR consumes $1.387 \times 10^{5}$ Joule with the pose-dependent lower-boundary method, and $1.554 \times 10^{5}$ Joule with the fixed lower-boundary method. In this example, the 
Table 5.3 Simulation results of the force distribution

\begin{tabular}{|l|c|c|c|c|c|c|c|c|c|}
\hline Cable serial number & $1^{\text {st }}$ & $2^{\text {nd }}$ & $3^{\text {rd }}$ & $4^{\text {th }}$ & $5^{\text {th }}$ & $6^{\text {th }}$ & $7^{\text {th }}$ & $8^{\text {th }}$ & Average \\
\hline \hline $\begin{array}{l}\text { Max cable force with pose- } \\
\text { dependent boundary (N) }\end{array}$ & 2473 & 3251 & 3692 & 3701 & 1085 & 520 & 760 & 874 & 2282 \\
\hline $\begin{array}{l}\text { Max cable force with } \\
\text { fixed boundary (N) }\end{array}$ & 2792 & 3510 & 4129 & 4137 & 1244 & 579 & 824 & 1041 & 2045 \\
\hline $\begin{array}{l}\text { Absolute difference of } \\
\text { cable force (N) }\end{array}$ & 319 & 260 & 437 & 436 & 159 & 59 & 64 & 167 & 237 \\
\hline $\begin{array}{l}\text { Relative difference of } \\
\text { cable force (\%) }\end{array}$ & 13 & 8 & 12 & 12 & 15 & 11 & 8 & 19 & 12 \\
\hline
\end{tabular}

pose-dependent lower-boundary method can save about $10.8 \%$ of energy.

In order to further test the proposed methods, similar simulations are made by using different kinds of trajectories (such as straight lines and circles) and different parameters of CDPRs (such as the end-effector mass, the cable diameter). From the results of these various simulations, the pose-dependent lower-boundary method can decrease cable forces and reduce energy consumption compared to the fixed lower-boundary method. For the sake of brevity, results are not presented in this chapter.

In fact, the efficiency of the pose-dependent lower-boundary method lies in its accurate calculation of the force boundary. Different cables at different poses have different requirements of force boundary to guarantee the cable's fundamental frequency to a certain value. The pose-dependent lower-boundary method calculate the lower-boundary for each driving cable at each pose of the end-effector along the trajectory, according to the requirement of cable dynamics. Therefore, this method can control the cable vibration to a desired level while not stretching the cables too much. Thus there is no unnecessary waste of motor torque or energy consumption.

\subsection{Summary of the chapter}

This chapter focuses on the force distribution of redundant actuated CDPRs. Force distribution method considering the effect of cable sag is firstly proposed. With cable sag, the kinematics and force distribution of CDPRs are coupled. The proposed method solves the coupling problem by using optimization algorithms. The purpose is to increase the computational accuracy of the cable forces, thus to improve the positioning accuracy of the end-effector and reduce the robot vibration.

A key issue is the determination of the lower-boundary of cable forces. In this chapter, 
the lower-boundary is calculated according to the cable's fundamental frequency. As the fundamental frequency is an important index to evaluate the cable dynamics and the CDPRs performance, the lower-boundary is related to the robot performances. With the proposed method, lower-boundary of cable forces can be determinated according to the performance requirement of CDPRs. After the lower-boundary is obtained, cable forces along a trajectory can be simulated by the proposed force distribution method. By analyzing the maximum cable force, design parameters such as the motor torque and etc. can be determinated.

Another important contribution of this chapter is the definition of the pose-dependent lower-boundary, which is computed in real-time for each driving cable at every pose of the end-effector according to the required fundamental frequency of cables. The pose-dependent lower-boundary method can guarantee the required performances of cables and CDPRs, while not stretch the cables too much. This method is significant to minimize the cable forces and energy consumption of CDPRs.

The proposed methods in this chapter are useful for the design and the simulation of CDPRs. In the future work, we plan to apply the proposed method on the optimization design of a CDPR prototype. 



\section{Chapter 6}

\section{Conclusions and perspectives}

\subsection{Conclusions}

The concept of Cable-Driven Parallel Robots has been pioneered for a few decades. Recently, more and more CDPR prototypes are build, some of which are already used or prepared to be used in real applications, such as the FSAT [Nan 2006; Kozak+ 2006; $\mathrm{Zi}+2008$ ], the CoGiRo [Nguyen+ 2013; Lamaury+ 2013] and the IPAnema [Pott+ 2013; Pott+ 2010]. These applications lead to the intensive researches of CDPRs. On the stiffness modeling and analysis of CDPRs, most previous researches use massless cable models that neglect the cable sag and the cable dynamics. In order to improve the static positioning accuracy and the dynamic trajectory tracking performances of CDPRs, complete cable models considering the effect of cable weight on the static cable profile and the effect of cable mass on the cable dynamics are used in this thesis. With these cable models, static and dynamic stiffness of CDPRs are analyzed. The main contributions of this thesis are listed as following:

\section{Static and dynamic modeling of CDPRs}

First of all, the static sagging cable model and the dynamic DSM cable model are introduced in details. For the static cable modeling, the most significant feature is the consideration of the cable elasticity and the effect of cable weight on the static cable profile. Thus the static sagging cable model is a complete and accurate model. For the dynamic cable modeling, a key result is the introduction of the DSM method. With DSM method, both cable elasticity and the effect of cable mass on the cable dynamics are taken into consideration. The dynamic cable characteristics can be expressed concisely and effectively by a stiffness matrix. 
Based on the static and dynamic cable models, the static and dynamic models of CDPRs are presented. The static stiffness model of CDPRs is proposed with considering both the cable elasticity and the effect of cable weight on the static cable profile, aiming to analyze and improve the static positioning accuracy of CDPRs, especially for the pick-and-place application. The pose error of the end-effector is defined and computed through the direct kinematic model of CDPRs. The static stiffness of CDPRs is evaluated by the variation of the pose error with external load.

The main purpose of the dynamic stiffness modeling of CDPRs is to analyze the effect of cable vibration on the dynamic behavior of CDPRs, aiming to answer the question that whether the effect of cable mass on the cable dynamics has a significant influence towards the system dynamics of CDPRs. To achieve this purpose, a new dynamic stiffness model of CDPRs is proposed:

- The dynamic stiffness matrix of CDPRs is firstly formulated. This dynamic matrix is an assemblage of the dynamic stiffness matrix of all the driving cables, which considers both the cable elasticity and the effect of cable mass on the cable dynamics;

- With this dynamic stiffness matrix, the oscillating equations of the end-effector around a static equilibrium are formulated through the Lagrange's equations;

- Dynamic response functions of CDPRs under a harmonic excitation are calculated, which contain all the dynamic informations of CDPRs.

This dynamic model of CDPRs considers the vibration of the end-effector, the cable vibration and their coupling. It is a complete model to describe the dynamics of CDPRs. Through this dynamic model, the natural frequencies of CDPRs can be identified and the coupling of cable dynamics and end-effector vibration can be analyzed.

\section{Experimental validation and stiffness analysis}

In order to verify the proposed models of cables and CDPRs, thus to improve the static and dynamic performances of CDPRs, experimental validations are made through two CDPR prototypes.

Firstly, static experimental validation is carried out through a 6-DOF CDPR prototype suspended by 6 cables to validate the static sagging cable model and the proposed static stiffness model of CDPRs. The variation of the pose error of the end-effector with the external load is studied by both simulations and experiments. Through the experiments, the sagging cable model is verified and the effect of external load on the the static stiffness of CDPRs 
is analyzed. It is shown that the sag-introduced flexibility is the main source of static robot stiffness with small external load, and the axial cable elasticity becomes the main source with big external load. The experimental results indicate the model relevance to predict the pose error due to the compliance of CDPRs. The proposed methods are quite useful for the design and optimization of CDPRs. Take a pick-and-place application as an example. When the end-effector is unloaded, cable sag is significant and the static stiffness of CDPRs is low. To assure a good positioning accuracy, the sagging cable model should be used in the controller. After cargoes are loaded on the end-effector, cable sag is reduced and robot stiffness improves. Once the total external load exceeds a threshold value, sag-introduced pose error can be neglected, and the simpler spring cable model is enough to ensure a good position accuracy. In addition, this threshold value can be calculated by the proposed models in this thesis.

Then dynamic experimental validation is made through a CDPR prototype the CoGiRo [Lamaury 2013] to verify the proposed dynamic stiffness model of CDPRs and analyze the coupling between the cable vibration and the end-effector vibration. The modal experiments, the free vibration experiments at an emergency stop during a trajectory and the dynamic experiments along a complete trajectory are performed. Results show the validity of the proposed dynamic models. It is also indicated that the vibration of CDPRs is a strong coupling of cable and end-effector vibrations when a periodic external excitation is applied, where cable dynamics can affect robot dynamics by changing and adding new resonances. It is the case in the applications such as the cable-driven wind tunnel, the giant cable-suspended telescope and the cable-suspended machine tool, cable dynamics where a periodic excitation source is directly or indirectly applied to cables. On the free vibration and trajectory vibration analysis of CDPRs, the main contribution in these responses is principally due to the rigidbody modes of the end-effector suspended on the global cable stiffness. The energy transfer between the cables vibration and the end-effector vibration is small.

\section{Force distribution of redundant actuated CDPRs}

Besides stiffness modeling and analysis, another contribution of this thesis is the application of the proposed methods on the force distribution of redundant actuated CDPRs. Force distribution method considering the effect of cable sag is proposed, aiming to increase the computational accuracy of the cable forces and thus to improve the positioning accuracy of the end-effector and reduce the robot vibration.

A key result is the determination of the lower-boundary of cable forces. In this thesis, the lower-boundary is calculated according to the cable's fundamental frequency. The 
fundamental frequency is an important index to evaluate the cable dynamics and the CDPRs performance. With the proposed method, lower-boundary of cable forces can be determinated according to the performance requirement of CDPRs. Another important contribution is the proposition of the pose dependent lower-boundary. The pose-dependent lower-boundary is not only associated with the cable performance but also related to the cable lengths. It is computed in real-time for each driving cable at every pose of the end-effector according to the required fundamental frequency of cables. It can guarantee the required performances of cables and CDPRs, while not stretch the cable too much.

Simulation results on a 6-DOF CDPR driven by 8 cables show the validity of the proposed force distribution method and indicate that the pose-dependent lower-boundary method is better than the fixed lower-boundary method on the minimization of the cable forces and energy consumption.

\subsection{Perspectives}

This thesis focuses on the static and dynamic stiffness modeling and analysis of CDPRs. A significant feature of this research is the introduction of the complete cable models and the application of these models on the stiffness analysis of CDPRs. Based on the above conclusions, perspectives for future works can be made as following:

\section{Design and optimization of CDPRs}

The proposed methods in this thesis are quite useful for the design, control and optimization of CDPRs. For example, different design parameters such as the cable diameter, the mass of the end-effector and the motor power can be optimized in order to improve the static and dynamic performances of CDPRs and to save the fabricating and operating costs in the same time. In future works, the proposed methods in this thesis should be employed in the optimal design of a CDPR prototype for an industrial application.

\section{Modeling of the joints of CDPRs}

In this thesis, the end points of the driving cables are assumed to be rigidly linked to the end-effector, and thus the compliance of the joints is neglected in the stiffness modeling of CDPRs. In fact, ball joints or universal joints are usually used to connect the cables and the end-effector in the fabrication of CDPRs. To improve analysis accuracy, the stiffness of the joints can be further considered and modeled. 


\section{Investigation of the damping}

The proposed dynamic model of CDPRs in this thesis only considers the constant viscous damping of cables, which is restrictive and does not depict the reality. Through a sensitivity analysis, a strong non-linearity of the damping through the frequency and a strong part of friction damping brought by the joints between the cables and the end-effector are expected. Although it does not affect the main objective of the modal analysis which is to identify the natural frequencies of the system, the damping behavior of CDPRs should be further investigated in future works to predict the energy dissipation and thus to improve the accuracy of the proposed dynamic models.

\section{Methods on the vibration suppression}

The vibration analysis of CDPRs is detailed in this thesis. Based on these results, methods on the vibration suppression can be further developed to reduce the vibration and to improve the dynamic tracking accuracy of CDPRs. For example, optimization can be performed in the trajectory generation to smooth the acceleration of the endeffector and thus to reduce vibration. In addition, active vibration canceling [Weber+ 2014] and input shaping methods can be further used in the controller to suppress the vibration of CDPRs. 



\section{References}

[Åkesson 1976]

[Albus+ 1992]

[Albus+ 1993]

[Algermissen+ 2005]

[Ansell 2005]

[Arsenault 2013]

[Banerjee 1997]

[Barrette+ 2005]

[Bedoustani+ 2008]
Bengt Å Åkesson. "PFVIBAT a computer program for plane frame vibration analysis by an exact method". In: International Journal for Numerical Methods in Engineering 10.6 (1976), pp. 12211231.

James Albus, Roger Bostelman, and Nicholas Dagalakis. "The NIST SPIDER, a robot crane". In: Journal of research of the National Institute of Standards and Technology 97.3 (1992), pp. 373385.

James Albus, Roger Bostelman, and Nicholas Dagalakis. "The NIST robocrane”. In: Journal of Robotic Systems 10.5 (1993), pp. 709-724.

Stephan Algermissen et al. "Applied robust control for vibration suppression in parallel robots". In: Proceedings of 22nd International Symposium on Automation and Robotics in Construction (ISARC), Ferrara, Italy. 2005.

Anders Ansell. "The dynamic element method for analysis of frame and cable type structures". In: Engineering structures 27.13 (2005), pp. 1906-1915.

Marc Arsenault. "Workspace and stiffness analysis of a threedegree-of-freedom spatial cable-suspended parallel mechanism while considering cable mass". In: Mechanism and Machine Theory 66 (2013), pp. 1-13.

JR Banerjee. "Dynamic stiffness formulation for structural elements: a general approach". In: Computers \& structures 63.1 (1997), pp. 101-103.

Guillaume Barrette and Clément M Gosselin. "Determination of the dynamic workspace of cable-driven planar parallel mechanisms". In: Journal of Mechanical Design 127.2 (2005), pp. 242248.

Yousef Babazadeh Bedoustani, Hamid D Taghirad, and Mohammad M Aref. "Dynamics analysis of a redundant parallel manipulator driven by elastic cables". In: Control, Automation, Robotics and Vision, 2008. ICARCV 2008. 10th International Conference on. IEEE. 2008, pp. 536-542. 
[Behzadipour+ 2006] Saeed Behzadipour and Amir Khajepour. "Stiffness of cable-based parallel manipulators with application to stability analysis". In: Journal of mechanical design 128.1 (2006), pp. 303-310.

[Bellet 1988]

[Berti+ 2013]

Didier Bellet. Cours de mécanique générale. Cepadues-editions, 1988.

Alessandro Berti, Jean-Pierre Merlet, and Marco Carricato. "Solving the direct geometrico-static problem of 3-3 cable-driven parallel robots by interval analysis: Preliminary results". In: CableDriven Parallel Robots. Springer, 2013, pp. 251-268.

[Borgstrom+ 2009] Per Henrik Borgstrom et al. "NIMS-PL: a cable-driven robot with self-calibration capabilities". In: Robotics, IEEE Transactions on 25.5 (2009), pp. 1005-1015.

[Bosscher+ 2005] Paul Bosscher, Robert L Williams, and Melissa Tummino. "A concept for rapidly-deployable cable robot search and rescue systems". In: ASME 2005 International Design Engineering Technical Conferences and Computers and Information in Engineering Conference. American Society of Mechanical Engineers. 2005, pp. 589598.

[Bosscher+ 2006] Paul Bosscher, Andrew T Riechel, and Imme Ebert-Uphoff. "Wrench feasible workspace generation for cable-driven robots". In: Robotics, IEEE Transactions on 22.5 (2006), pp. 890-902.

[Bostelman+ 1994] Roger Bostelman et al. "Applications of the NIST RoboCrane". In: Proceedings of the 5th International Symposium on Robotics and Manufacturing. 1994, pp. 14-18.

[Briot+ 2007]

Sébastien Briot et al. "Contribution to the mechanical behavior improvement of the Robotic navigation device Surgiscope ${ }^{\circledR}$ ". In: ASME 2007 International Design Engineering Technical Conferences and Computers and Information in Engineering Conference. American Society of Mechanical Engineers. 2007, pp. 653-661.

[Bruckmann+ 2006] Tobias Bruckmann, Andreas Pott, and Manfred Hiller. "Calculating force distributions for redundantly actuated tendon-based Stewart platforms". In: Advances in Robot Kinematics. Springer, 2006, pp. 403-412.

[Bruckmann+ 2010] Tobias Bruckmann, Christian Sturm, and Wildan Lalo. "Wire robot suspension systems for wind tunnels". In: Wind Tunnels and Experimental Fluid Dynamics Research (2010), pp. 29-50.

[Carbone 2011] Giuseppe Carbone. "Stiffness analysis and experimental validation of robotic systems". In: Frontiers of Mechanical Engineering 6.2 (2011), pp. 182-196.

[Clavel 1991]

Reymond Clavel. "Conception d'un robot parallèle rapide à 4 degrés de liberté". Thèse de doctorat. École Polytechnique Fédérale de Lausanne, 1991. 
[Courteille+ 2009]

[Dagalakis+ 1989]

[Dallej+ 2012]

[Deblaise+ 2006]

[Diao+ 2009]

$[\mathrm{Du}+2012]$

[Du+ 2013]

[Dunlop+ 1999]

[ElKhasawneh+ 1999]

[Fang+ 2004]

[Gallina+ 2002]

[Gexue+ 2004]

[Gosselin 1990]
Eric Courteille, Dominique Deblaise, and Patrick Maurine. "Design optimization of a Delta-like parallel robot through global stiffness performance evaluation”. In: Intelligent Robots and Systems, 2009. IROS 2009. IEEE/RSJ International Conference on. IEEE. 2009, pp. 5159-5166.

Nicholas G Dagalakis et al. "Stiffness study of a parallel link robot crane for shipbuilding applications". In: Journal of Offshore Mechanics and Arctic Engineering 111.3 (1989), pp. 183-193.

Tej Dallej et al. "Vision-based modeling and control of largedimension cable-driven parallel robots". In: Intelligent Robots and Systems (IROS), 2012 IEEE/RSJ International Conference on. IEEE. 2012, pp. 1581-1586.

Dominique Deblaise, Xavier Hernot, and Patrick Maurine. "A systematic analytical method for PKM stiffness matrix calculation". In: Robotics and Automation, 2006. ICRA 2006. Proceedings 2006 IEEE International Conference on. IEEE. 2006, pp. 4213-4219.

Xiumin Diao and Ou Ma. "Vibration analysis of cable-driven parallel manipulators". In: Multibody System Dynamics 21.4 (2009), pp. 347-360.

Jingli Du et al. "Dynamic analysis of cable-driven parallel manipulators with time-varying cable lengths". In: Finite Elements in Analysis and Design 48.1 (2012), pp. 1392-1399.

Jingli Du, Wen Ding, and Hong Bao. "Cable vibration analysis for large workspace cable-driven parallel manipulators". In: CableDriven Parallel Robots. Springer, 2013, pp. 437-449.

GR Dunlop and TP Jones. "Position analysis of a two DOF parallel mechanism - the Canterbury tracker". In: Mechanism and Machine Theory 34.4 (1999), pp. 599-614.

Bashar S El-Khasawneh and Placid M Ferreira. "Computation of stiffness and stiffness bounds for parallel link manipulators". In: International Journal of Machine Tools and Manufacture 39.2 (1999), pp. 321-342.

Shiqing Fang et al. "Motion control of a tendon-based parallel manipulator using optimal tension distribution". In:Mechatronics, IEEE/ASME Transactions on 9.3 (2004), pp. 561-568.

Paolo Gallina and Giulio Rosati. "Manipulability of a planar wire driven haptic device". In: Mechanism and Machine Theory 37.2 (2002), pp. 215-228.

Ren Gexue et al. "On vibration control with Stewart parallel mechanism”. In: Mechatronics 14.1 (2004), pp. 1-13.

Clement Gosselin. "Stiffness mapping for parallel manipulators". In: Robotics and Automation, IEEE Transactions on 6.3 (1990), pp. 377-382. 
[Gosselin+ 2011]

[Gough 1957]

[Gouttefarde+ 2006]

[Gouttefarde+ 2007]

[Gouttefarde+ 2012]

[Hassan+ 2008]

[Hesselbach+ 2004]

[Heyden+ 2006]

[Hiller+ 2005]

[Hughes 2012]

[Irvine 1978]

[Irvine 1992]

[Kang+ 2005]

[Kawamura+ 1993]
Clément Gosselin and Martin Grenier. "On the determination of the force distribution in overconstrained cable-driven parallel mechanisms". In: Meccanica 46.1 (2011), pp. 3-15.

V. E. Gough. "Contribution to discussion of papers on research in Automobile Stability, Control and Tyre performance". In: Proc. Auto Div. Inst. Mech. Eng. (1956-1957), pp. 392-394.

Marc Gouttefarde and Clément M Gosselin. "Analysis of the wrench-closure workspace of planar parallel cable-driven mechanisms". In: Robotics, IEEE Transactions on 22.3 (2006), pp. 434445.

Marc Gouttefarde, J-P Merlet, and David Daney. "Wrench-feasible workspace of parallel cable-driven mechanisms". In: Robotics and Automation, 2007 IEEE International Conference on IEEE. 2007, pp. 1492-1497.

Marc Gouttefarde et al. "Simplified static analysis of large dimension parallel cable-driven robots". In: Robotics and Automation (ICRA), 2012 IEEE International Conference on. IEEE. 2012, pp. 2299-2305.

Mahir Hassan and Amir Khajepour. "Optimization of actuator forces in cable-based parallel manipulators using convex analysis". In: Robotics, IEEE Transactions on 24.3 (2008), pp. 736-740.

Jürgen Hesselbach et al. "Aspects on design of high precision parallel robots". In: Assembly Automation 24.1 (2004), pp. 49-57.

Thomas Heyden and Christoph Woernle. "Dynamics and flatnessbased control of a kinematically undetermined cable suspension manipulator". In: Multibody System Dynamics 16.2 (2006), pp. 155177.

Manfred Hiller et al. "Design, analysis and realization of tendonbased parallel manipulators". In: Mechanism and Machine Theory 40.4 (2005), pp. 429-445.

Thomas JR Hughes. The finite element method: linear static and dynamic finite element analysis. Courier Dover Publications, 2012.

H Max Irvine. "Free vibrations of inclined cables". In: Journal of the Structural Division 104.2 (1978), pp. 343-347.

H.M. Irvine. Cable Structures. Dover books on engineering. Dover Publications, 1992. ISBN: 9780486671277.

Bongsoo Kang and James K Mills. "Vibration control of a planar parallel manipulator using piezoelectric actuators". In: Journal of Intelligent and Robotic Systems 42.1 (2005), pp. 51-70.

Sadao Kawamura and Ken Ito. "A new type of master robot for teleoperation using a radial wire drive system". In: Intelligent Robots and Systems' 93, IROS'93. Proceedings of the 1993 IEEE/RSJ International Conference on. Vol. 1. IEEE. 1993, pp. 55-60. 
[Kawamura+ 1995] Sadao Kawamura et al. "Development of an ultrahigh speed robot FALCON using wire drive system". In: Robotics and Automation, 1995. Proceedings., 1995 IEEE International Conference on. Vol. 1. IEEE. 1995, pp. 215-220.

[Kawamura+ 2000] Sadao Kawamura, Hitoshi Kino, and Choe Won. "High-speed manipulation by using parallel wire-driven robots". In: Robotica 18.01 (2000), pp. 13-21.

[Khosravi+ 2013] MA Khosravi and HD Taghirad. "Robust PID control of cabledriven robots with elastic cables". In: Robotics and Mechatronics (ICRoM), 2013 First RSI/ISM International Conference on. IEEE. 2013, pp. 331-336.

$[\mathrm{Kim}+2001]$

Jonghwa Kim and Sung Pil Chang. "Dynamic stiffness matrix of an inclined cable". In: Engineering structures 23.12 (2001), pp. 16141621.

[Koevermans+ 1975] WP Koevermans and CJ Jansen. "Design and performance of the four dof motion system of the NLR research flight simulator". In: Proc. of AGARD Conf. 198. 1975, pp. 17-1.

[Koloušek+ 1973] Vladimír Koloušek and Reuben Fraser McLean. Dynamics in engineering structures. Butterworths, 1973.

[Korayem+ 2007] Moharam Habibnejad Korayem, Mahdi Bamdad, and M Saadat. "Workspace analysis of cable-suspended robots with elastic cable". In: Robotics and Biomimetics, 2007. ROBIO 2007. IEEE International Conference on. IEEE. 2007, pp. 1942-1947.

$[$ Kozak+ 2004]

Kris Kozak, Imme Ebert-Uphoff, and William Singhose. "Locally linearized dynamic analysis of parallel manipulators and application of input shaping to reduce vibrations". In: Journal of Mechanical design 126.1 (2004), pp. 156-168.

[Kozak+ 2006]

Kris Kozak, Qian Zhou, and Jinsong Wang. "Static analysis of cable-driven manipulators with non-negligible cable mass". In: Robotics, IEEE Transactions on 22.3 (2006), pp. 425-433.

[Lahouar+ 2009] Samir Lahouar et al. "Collision free path-planning for cable-driven parallel robots". In: Robotics and Autonomous Systems 57.11 (2009), pp. 1083-1093.

[Lamaury 2013] Johann Lamaury. "Contribution à la commande des robots parallèes à câbles à redondance d'actionnement". français. Thèse de doctorat. Montpellier, France: Laboratoire d'informatique, de robotique et de micro-électronique, 2013.

[Lamaury+ 2013] Johann Lamaury and Marc Gouttefarde. "Control of a large redundantly actuated cable-suspended parallel robot". In: Robotics and Automation (ICRA), 2013 IEEE International Conference on . IEEE. 2013, pp. 4659-4664.

[Leung 1978] Andrew Yee-Tak Leung. "An accurate method of dynamic condensation in structural analysis". In: International Journal for Numerical Methods in Engineering 12.11 (1978), pp. 1705-1715. 
[Lim+ 2011]

[Ma+ 2005]

[Mao+ 2012]

[Merlet 2006]

[Merlet 2010]

[Merlet+ 2010]

[Miermeister+ 2012]

[Mikelsons+ 2008]

[Mitsuta+ 1994]

[Nabat 2007]

[Nan 2006]

[Nguyen+ 2013]

[Nguyen+ 2015]
Wen Bin Lim et al. "A generic force-closure analysis algorithm for cable-driven parallel manipulators". In: Mechanism and Machine Theory 46.9 (2011), pp. 1265-1275.

Ou Ma and Xiumin Diao. "Dynamics analysis of a cable-driven parallel manipulator for hardware-in-the-loop dynamic simulation”. In: Advanced Intelligent Mechatronics. Proceedings, 2005 IEEE/ASME International Conference on. IEEE. 2005, pp. 837842.

Ying Mao and Sunil Kumar Agrawal. "Design of a cable-driven arm exoskeleton (CAREX) for neural rehabilitation". In: Robotics, IEEE Transactions on 28.4 (2012), pp. 922-931.

J-P Merlet. Parallel robots. Vol. 128. Springer, 2006.

J-P Merlet. "MARIONET, a family of modular wire-driven parallel robots". In: Advances in Robot Kinematics: Motion in Man and Machine. Springer, 2010, pp. 53-61.

J-P Merlet and David Daney. "A portable, modular parallel wire crane for rescue operations". In: Robotics and Automation (ICRA), 2010 IEEE International Conference on. IEEE. 2010, pp. 28342839.

Philipp Miermeister, Andreas Pott, and Alexander Verl. "Autocalibration method for overconstrained cable-driven parallel robots". In: Robotics; Proceedings of ROBOTIK 2012; 7th German Conference on. VDE. 2012, pp. 1-6.

Lars Mikelsons et al. "A real-time capable force calculation algorithm for redundant tendon-based parallel manipulators". In: Robotics and Automation, 2008. ICRA 2008. IEEE International Conference on. IEEE. 2008, pp. 3869-3874.

Shinji Mitsuta et al. "Active vibration control of structures arranged in parallel". In: JSME international journal. Ser. C, Dynamics, control, robotics, design and manufacturing 37.3 (1994), pp. 436443.

Vincent Nabat. "Robots parallèles à nacelle articulée: du concept à la solution industrielle pour le pick-and-place". Thèse de doctorat. Montpellier 2, 2007.

Rendong Nan. "Five hundred meter aperture spherical radio telescope (FAST)". In: Science in China series G 49.2 (2006), pp. 129148.

Dinh Quan Nguyen et al. "On the simplifications of cable model in static analysis of large dimension cable-driven parallel robots". In: Intelligent Robots and Systems (IROS), 2013 IEEE/RSJ International Conference on. IEEE. 2013, pp. 928-934.

Dinh Quan Nguyen and Marc Gouttefarde. "On the Improvement of Cable Collision Detection Algorithms". In: Cable-Driven Parallel Robots. Springer, 2015, pp. 29-40. 
[Oh+ 2005]

[Piras+ 2005]

[Pott 2010]

[Pott+ 2009]

[Pott+ 2010]

[Pott+ 2013]

[Pusey+ 2004]

[Rao+ 1995]

[Riehl 2011]

[Riehl+ 2009]

[Rosati+ 2005]

[Sandretto+ 2013a]
So-Ryeok Oh and Sunil Kumar Agrawal. "Cable suspended planar robots with redundant cables: controllers with positive tensions". In: Robotics, IEEE Transactions on 21.3 (2005), pp. 457-465.

Gabriel Piras, WL Cleghorn, and JK Mills. "Dynamic finite-element analysis of a planar high-speed, high-precision parallel manipulator with flexible links". In: Mechanism and Machine Theory 40.7 (2005), pp. 849-862.

Andreas Pott. "An algorithm for real-time forward kinematics of cable-driven parallel robots". In: Advances in Robot Kinematics: Motion in Man and Machine. Springer, 2010, pp. 529-538.

Andreas Pott, Tobias Bruckmann, and Lars Mikelsons. "Closedform force distribution for parallel wire robots". In: Computational Kinematics. Springer, 2009, pp. 25-34.

Andreas Pott, Christian Meyer, and Alexander Verl. "Large-scale assembly of solar power plants with parallel cable robots". In: Robotics (ISR), 2010 41st International Symposium on and 2010 6th German Conference on Robotics (ROBOTIK). VDE. 2010, pp. 1-6.

Andreas Pott et al. "IPAnema: a family of cable-driven parallel robots for industrial applications". In: Cable-Driven Parallel Robots. Springer, 2013, pp. 119-134.

Jason Pusey et al. "Design and workspace analysis of a 6-6 cablesuspended parallel robot". In: Mechanism and Machine Theory 39.7 (2004), pp. 761-778.

Singiresu S Rao and Fook Fah Yap. Mechanical vibrations. Vol. 4. Addison-Wesley New York, 1995.

Nicolas Riehl. "Modélisation et design de robots parallèes à câbles de grande dimension". français. Thèse de doctorat. Montpellier, France: Laboratoire d'informatique, de robotique et de microélectronique, 2011.

Nicolas Riehl et al. "Effects of non-negligible cable mass on the static behavior of large workspace cable-driven parallel mechanisms". In: Robotics and Automation, 2009. ICRA'09. IEEE International Conference on. IEEE. 2009, pp. 2193-2198.

Giulio Rosati et al. "Design of a new 5 dof wire-based robot for rehabilitation". In: Rehabilitation Robotics, 2005. ICORR 2005. 9th International Conference on. IEEE. 2005, pp. 430-433.

Julien Alexandre dit Sandretto, David Daney, and Marc Gouttefarde. "Calibration of a fully-constrained parallel cable-driven robot". In: Romansy 19-Robot Design, Dynamics and Control. Springer, 2013, pp. 77-84. 
[Sandretto+ 2013b] Julien Alexandre dit Sandretto, Gilles Trombettoni, and David Daney. "Confirmation of hypothesis on cable properties for cabledriven robots". In: New Trends in Mechanism and Machine Science. Springer, 2013, pp. 85-93.

[Starossek 1991a] Uwe Starossek. Boundary induced vibration and dynamic stiffness of a sagging cable. ISD Universität Stuttgart, 1991.

[Starossek 1991b] Uwe Starossek. "Dynamic stiffness matrix of sagging cable”. In: Journal of engineering mechanics 117.12 (1991), pp. 2815-2828.

[Stewart 1965]

Doug Stewart. "A platform with six degrees of freedom”. In: Proceedings of the institution of mechanical engineers 180.1 (1965), pp. 371-386.

[Surdilovic+ 2007] Dragoljub Surdilovic, Jinyu Zhang, and Rolf Bernhardt. "STRINGMAN: Wire-robot technology for safe, flexible and human-friendly gait rehabilitation". In: Rehabilitation Robotics, 2007. ICORR 2007. IEEE 10th International Conference on. IEEE. 2007, pp. 446453.

[Tadokoro+ 1999] Satoshi Tadokoro et al. "A portable parallel manipulator for search and rescue at large-scale urban earthquakes and an identification algorithm for the installation in unstructured environments". In: Intelligent Robots and Systems, 1999. IROS'99. Proceedings. 1999 IEEE/RSJ International Conference on. Vol. 2. IEEE. 1999, pp. 1222-1227.

[Tang+ 2013]

Ao Fei Tang et al. "Vibration Analysis of Tendon-Based Parallel Robot for Processing”. In: Advanced Materials Research 655 (2013), pp. 1086-1091.

[Vafaei+ 2011] Alaleh Vafaei, Mohammad A Khosravi, and Hamid D Taghirad. "Modeling and control of cable driven parallel manipulators with elastic cables: singular perturbation theory". In: Intelligent Robotics and Applications. Springer, 2011, pp. 455-464.

[Verhoeven+ 1998] Richard Verhoeven, Manfred Hiller, and Satoshi Tadokoro. "Workspace, stiffness, singularities and classification of tendon-driven stewart platforms". In: Advances in robot kinematics: Analysis and Control. Springer, 1998, pp. 105-114.

[Von Zitzewitz+ 2010] Joachim Von Zitzewitz et al. "Forward kinematics of redundantly actuated, tendon-based robots". In: Intelligent Robots and Systems (IROS), 2010 IEEE/RSJ International Conference on. IEEE. 2010, pp. 2289-2294.

[Wang+ 2006] Xiaoyun Wang and James K Mills. "Dynamic modeling of a flexible-link planar parallel platform using a substructuring approach". In: Mechanism and Machine Theory 41.6 (2006), pp. 671687. 
[Weber+ 2014]

[Weck+ 2002]

[Yuan+ 2014]

[Yuan+ 2015]

[Yun+ 2010]

[Zhang+ 2008]

[Zhou 1996]

$[\mathrm{Zi}+2008]$
Xavier Weber, Loic Cuvillon, and Jacques Gangloff. "Active vibration canceling of a cable-driven parallel robot using reaction wheels". In: Intelligent Robots and Systems (IROS 2014), 2014 IEEE/RSJ International Conference on. IEEE. 2014, pp. 17241729.

Manfred Weck and Dirk Staimer. "Parallel kinematic machine tools-current state and future potentials". In: CIRP Annals-Manufacturing Technology 51.2 (2002), pp. 671-683.

Han Yuan, Eric Courteille, and Dominique Deblaise. "Elastodynamic analysis of cable-driven parallel manipulators considering dynamic stiffness of sagging cables". In: Robotics and Automation (ICRA), 2014 IEEE International Conference on. IEEE. 2014, pp. 4055-4060.

Han Yuan, Eric Courteille, and Dominique Deblaise. "Static and dynamic stiffness analyses of cable-driven parallel robots with nonnegligible cable mass and elasticity". In: Mechanism and Machine Theory 85 (2015), pp. 64-81.

Yuan Yun and Yangmin Li. "Design and analysis of a novel 6DOF redundant actuated parallel robot with compliant hinges for high precision positioning". In: Nonlinear Dynamics 61.4 (2010), pp. 829-845.

Xuping Zhang, James K Mills, and William L Cleghorn. "Vibration control of elastodynamic response of a 3-PRR flexible parallel manipulator using PZT transducers". In: Robotica 26.05 (2008), pp. 655-665.

Wan-E. Zhou. "The dynamic stiffness method". Doctoral thesis. Civil and Structural Engineering, 1996.

Bin $\mathrm{Zi}$ et al. "Dynamic modeling and active control of a cablesuspended parallel robot". In: Mechatronics 18.1 (2008), pp. 112. 



\section{Appendix A}

\section{Experimental equipments}

1) The electrodynamic vibration shaker

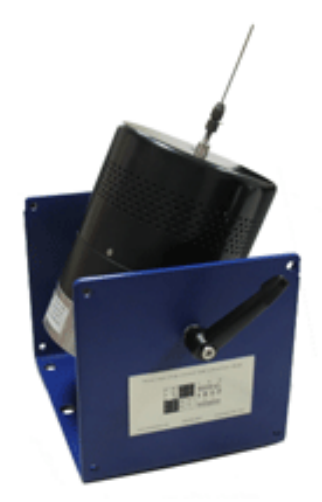

Fig. A.1 The electrodynamic vibration shaker

As shown in fig. A.1, an electrodynamic vibration shaker ${ }^{1}$ is used. It is a lightweight electrodynamic modal exciter, capable of providing $440 \mathrm{~N}$ of peak force excitation in a small footprint weighing just $15 \mathrm{~kg}$. With a useful frequency range beyond $5400 \mathrm{~Hz}$, it is suitable for structural testing and experimental modal analysis applications, using random, burst random, sine dwell or chirp excitation signals.

2) The Nikon K-600 camera system

As shown in fig. A.2, a measurement device (Nikon Metrology K600-10 system ${ }^{2}$ ) is used. It is based on three CCD linear cameras and 3 infra-red light active LEDs. In the following experiments, these 3 LEDs are attached to the end-effector. With this system,

\footnotetext{
${ }^{1}$ This is a product of The Modal Shop ${ }^{\circledR}$ company, model 2100E11. http://www.modalshop.com/default.asp

${ }^{2}$ This is a product of Nikon ${ }^{\circledR}$ company: http://www.nikonmetrology.com
} 


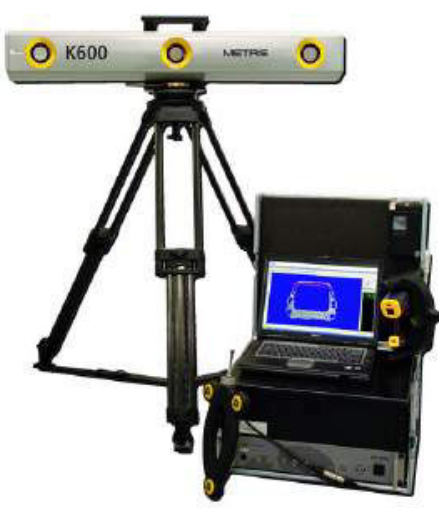

Fig. A.2 The Nikon K-600 camera system

the poses of the end-effector (both position and orientation) can be measured. The system has a position measuring accuracy up to $\pm 37 \mu \mathrm{m}$ for a single point.

3) Sensors

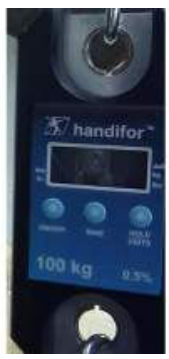

(a) The dynamometer

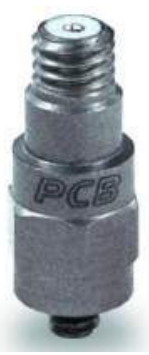

(b) The accelerometer

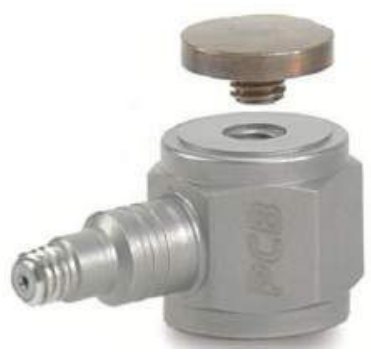

(c) The force sensor

Fig. A.3 Sensors in the experiments

Figure A. 3 shows the sensors used in the following experiments. Several dynamometers ${ }^{3}$ shown in fig. A.3a are used to measure the static cable force. These dynamometers have a measuring range of $0 \sim 1000 \mathrm{~N}$ and a measuring precision of $\pm 3 \mathrm{~N}$. Three triaxial accelerometers ${ }^{4}$ shown in fig. A.3b are used, with a sensitivity of $100 \mathrm{mV} / \mathrm{g}$ and a weight of $2 \mathrm{gm}$. A force sensor ${ }^{5}$ shown in fig. A.3c is used, with a sensitivity of $224.82 \mathrm{mV} / \mathrm{kN}$ and a measurement range of $\pm 22.24 \mathrm{kN}$.

4) The data acquisition system

\footnotetext{
${ }^{3}$ This is a product of Handifor ${ }^{T M}$

${ }^{4}$ This is a product of PCB company, model PCB-352C68. http://www.pcb.com/Products.aspx?m=352C68

${ }^{5}$ This is a product of PCB company, model PCB-208C05. http://www.pcb.com/Products.aspx?m=208C05
} 


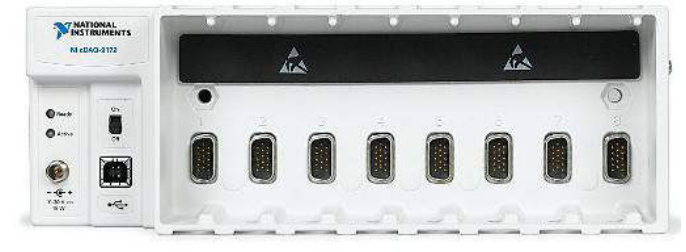

(a) The NI CompactDAQ chassis

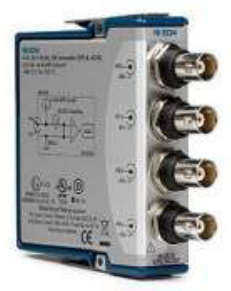

(b) The NI analog input card

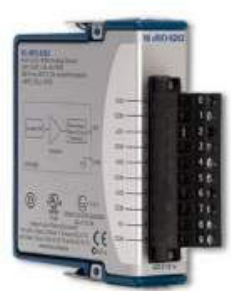

(c) The NI analog output card

Fig. A.4 The data acquisition system

The portable data acquisition system is a CompactDAQ ${ }^{6}$ with 8 slots shown in fig. A.4a. It integrates connectivity and signal conditioning into modular $\mathrm{I} / \mathrm{O}$ for directly interfacing to any sensor or signal. The modules used for the tests provide built-in signal conditioning such as amplification, filtering and excitation. Three accelerometer modules ${ }^{7}$ shown in fig. A.4b are used. Each of them has 4 channels, with a $51.2 \mathrm{kS} / \mathrm{s}$ per channel maximum sampling rate and a 24-bit resolution. An analog output modules ${ }^{8}$ shown in fig. A.4c is used. It has 4 channels, with a $100 \mathrm{kS} / \mathrm{s}$ per channel simultaneous analog output and a 16-bit resolution.

\footnotetext{
${ }^{6}$ This is a product of NI company, model NI-9172. http://sine.ni.com/nips/cds/view/p/lang/en/nid/202545

${ }^{7}$ This is a product of NI company, model NI-9234. http://sine.ni.com/nips/cds/view/p/lang/en/nid/208802

${ }^{8}$ This is a product of NI company, model NI-9263. http://sine.ni.com/nips/cds/view/p/lang/en/nid/208806
} 

INSA de RENNES

Service des Formations

AVIS DU JURY SUR LA REPRODUCTION DE LA THESE SOUTENUE

Titre de la thèse:

Static and dynamic stiffness analysis of cable-driven parallel robots

Nom Prénom de l'auteur : YUAN HAN

Membres du jury:

- Monsieur GANGLOFF jacques

- Monsieur RAGNEAU ERIC

- Monsieur COURTEliliE Entc

- Monsieur CARO Stéphane

- Monsieur BARADAT Cédric

- Monsiear DUMONT Georges

- Monsieur GOUTTEFARDE Marc

monsieur SAMPER Serge

Président dujury: Secge Sampo

Date de la soutenance : 11 Mars 2015

Reproduction de la these soutenue

Thèse potvant être reproduite en l'état.

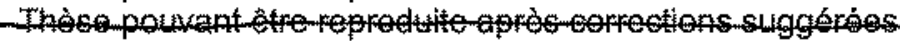

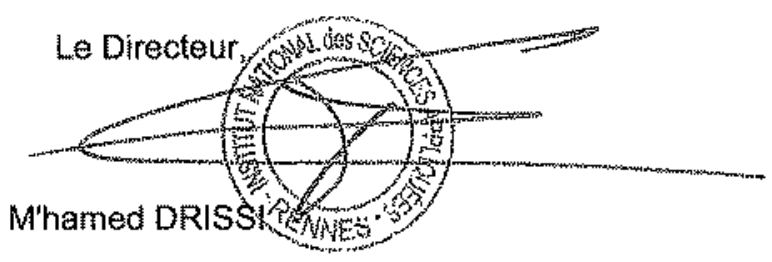

Fait à Rennes, le 11 Mars 2015

Signature du prósident de jury

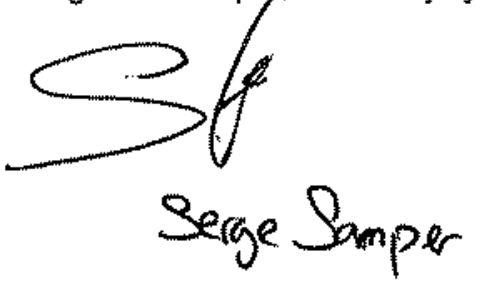




Résumé

Cette thèse contribue à l'analyse des raideurs statique et dynamique des robots parallèles à câbles dans un objectif d'amélioration de la précision de positionnement statique et de la précision de suivi de trajectoire.

Les modélisations statique et dynamique proposées des câbles considèrent l'effet du poids du câble sur son profil et l'effet de masse du câble sur la dynamique de ce dernier. Sur la base du modèle statique de câble proposé, l'erreur de pose statique au niveau de l'organe terminal du robot est définie et sa variation en fonction de la charge externe appliquée est utilisée pour évaluer la raideur statique globale de la structure. Un nouveau modèle dynamique vibratoire de robots à câbles est proposé en considérant le couplage de la dynamique des câbles avec les vibrations de l'organe terminal.

Des validations expérimentales sont réalisées sur des prototypes de robots à câbles. Une série d'expériences de statique, d'analyses modales, d'analyses en régime libre et de suivi de trajectoire sont réalisées. Les modèles statiques et dynamiques proposés sont confirmés. Les dynamiques des câbles et du robot ainsi que leur couplage sont discutées montrant la pertinence des modèles développés pour l'amélioration des performances des robots à câbles en termes de design et le contrôle.

Outre l'analyse des raideurs statique et dynamique, les modèles proposés sont appliqués dans l'amélioration du calcul de la distribution des efforts dans les câbles des robots redondants. Une nouvelle méthode de calcul de la distribution des efforts dans les câbles basée sur la détermination de la limite inférieure des forces dans les câbles est présentée. La prise en compte de la dépendance à la position dans l'espace de travail permet de limiter les efforts dans les câbles et ainsi d'améliorer l'efficience des robots d'un point de vue énergétique.
This thesis contributes to the analysis of the static and dynamic stiffness of cable-driven parallel robots (CDPRs) aiming to improve the static positioning accuracy and the trajectory tracking accuracy.

The proposed static and dynamic cable modeling considers the effect of cable weight on the cable profile and the effect of cable mass on the cable dynamics. Based on the static cable model, the static pose error of the end-effector is defined and the variation of the end-effector pose error with the external load is used to evaluate the static stiffness of CDPRs. A new dynamic model of CDPRs is proposed with considering the coupling of the cable dynamics and the end-effector vibrations.

Experimental validations are carried out on CDPR prototypes. Static experiments, modal experiments, free vibration experiments and trajectory experiments are performed. The proposed static and dynamic models are verified. Cable dynamics, robot dynamics and their coupling are discussed. Results show the relevance of the proposed models on improving the performances of CDPRs in terms of design and control.

Besides stiffness analysis, the proposed models are applied on the force distribution of redundant actuated CDPRs. A new method on the calculation of the cable forces is proposed, where the determination of the lower-boundary of the cable forces is presented. The consideration of the pose-dependence of the lower force boundary can minimize the cable forces and improve the energy efficiency of CDPRs. 